\title{
Novel insights into speech production networks of adults with developmental stuttering as revealed by analyses of speech intention, syllable frequency, and long-term therapy effects
}

\author{
Dissertation \\ zur Erlangung des mathematisch-naturwissenschaftlichen Doktorgrades \\ "Doctor rerum naturalium" \\ der Georg-August-Universität Göttingen
}

im Promotionsstudiengang Behavior and Cognition der Georg-August University School of Science (GAUSS)

vorgelegt von

Alexandra Marina Korzeczek

aus Braunschweig, Deutschland

Göttingen, 2021 


\section{Betreuungsausschuss}

Prof. Dr. Martin Sommer

Klinik für Klinische Neurophysiologie, Klinik für Neurologie, Universitätsmedizin

Göttingen

Prof. Dr. Melanie Wilke

MR-Forschung in der Neurologie und Psychiatrie, Abteilung Kognitive Neurologie, Universitätsmedizin Göttingen

Prof. Dr. Nivedita Mani

Forschungsgruppe "Sprachpsychologie", Georg-Elias-Müller-Institut für Psychologie, Georg-August-Universität Göttingen

\section{Mitglieder der Prüfungskommission}

Referent: Prof. Dr. Martin Sommer

Klinik für Klinische Neurophysiologie, Klinik für Neurologie, Universitätsmedizin

Göttingen

Korreferent: Prof. Dr. Melanie Wilke

MR-Forschung in der Neurologie und Psychiatrie, Abteilung Kognitive Neurologie, Universitätsmedizin Göttingen

Weitere Mitglieder der Prüfungskommission:

Prof. Dr. Nivedita Mani

Forschungsgruppe "Sprachpsychologie", Georg-Elias-Müller-Institut für Psychologie, Georg-August-Universität Göttingen

Dr. Audrey Bürki-Foschini

Cognitive Science, Department of Linguistics, University of Potsdam

Dr. Roberto Goya-Maldonado

Labor für Systemische Neurowissenschaften und Bildgebung in der Psychiatrie (SNIP), Universitätsmedizin Göttingen

Prof. Dr. Annekathrin Schacht

Affektive Neurowissenschaft und Psychophysiologie, Georg-Elias-Müller-Institute für Psychologie, Georg-August-Universität Göttingen

Tag der mündlichen Prüfung: 12.02.2021 



\section{Acknowledgments}

I want to sincerely thank my supervisor Martin Sommer for giving me the opportunity to delve into the topic of speech production in stuttering and for supporting me during this journey. I appreciated the weekly meetings and our clinical collaboration for the stuttering council.

Furthermore, I wish to express my thankfulness to all participants, especially all the participants with developmental stuttering. Their spirit and dedication to travel across Germany to partake in my study was inspiring and motivating.

I am grateful to Nicole E. Neef and Joana Cholin for their intellectual contributions and motivational support. I thank Melanie Wilke and Nivedita Mani for their co-supervision and for the insightful discussions that fostered my progress. I am thankful to Walter Paulus for his support and discussions on brain stimulation.

Additionally, I am grateful to my direct collaborators Jana Wiechmann, Katharina Hammers, Devran Yildirim, Iris Steinman and Annika Primaßin for contributing to my projects and working together. Special thanks to my colleagues, Barbara, Christina, Elina, Aditya, Albert, Birtan, Daniel, David, Faizal, Gabriel, Ivan and Zsolt for the engaging discussions, their help and the time we spent together.

Many thanks to Marion Kurze, Manuel Hewitt and further members of the Department of Clinical Neurophysiology for all the administrative and technical support, which aided my research projects. For financial support and for great workshops, I would like to thank the Leibniz-Science Campus "Primate Cognition”. I am grateful to Rebecca Jürgens, our coordinator of Behavior and Cognition, for all the support during the past three years.

I thank my family and my friends. Without your (emotional) support, this thesis would not have been possible. 


\section{Table of Contents}

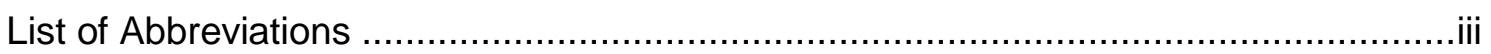

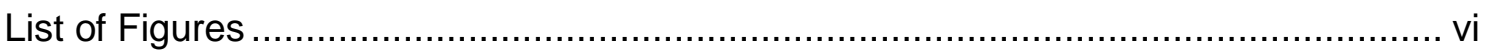

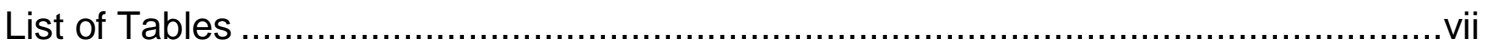

List of Tables in Appendices .................................................................... vii

List of Figures in Appendices .............................................................................. ix

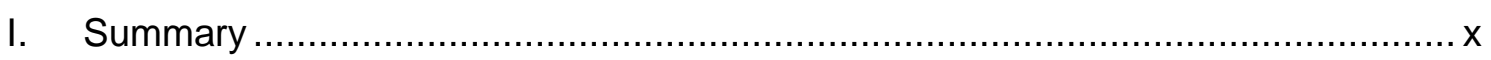

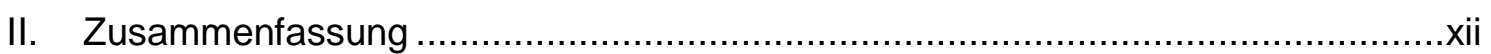

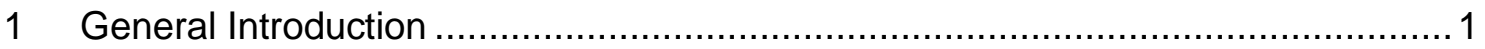

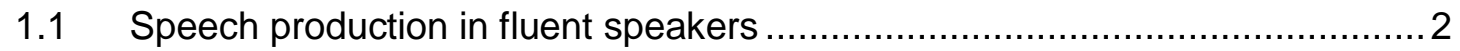

1.2 Medical background of stuttering ........................................................... 4

1.2.1 Definition ................................................................................. 4

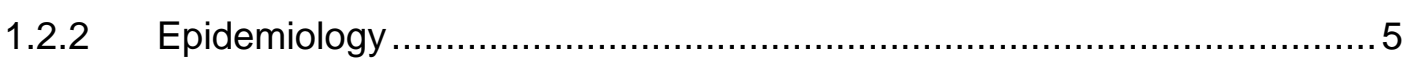

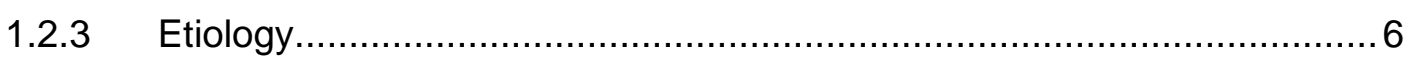

1.3 Theories on stuttering emergence …................................................... 8

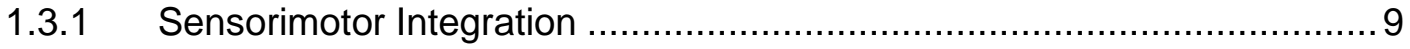

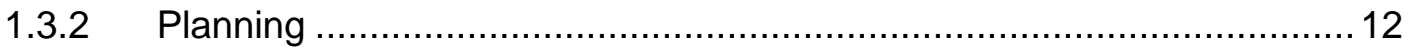

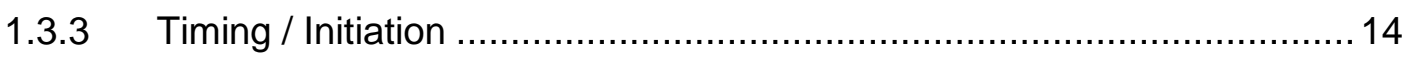

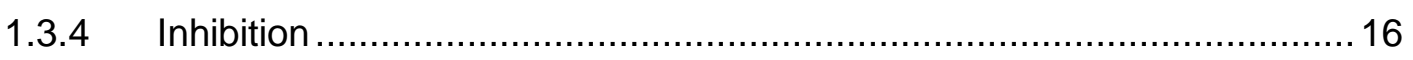

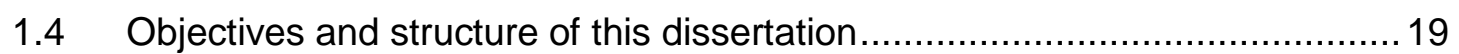

2 Increased right frontal beta power during speech intention in severe stuttering ...21

3 Effects of word length and syllable frequency in adults with developmental stuttering 54

4 Fluent speech: neural basis of sensorimotor plasticity in developmental stuttering 88

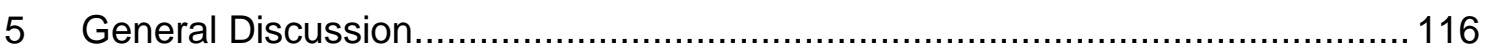

5.1 Sensorimotor integration - preventing or inducing stuttering events? .......... 118

5.1.1 Normal sensorimotor preparation in stuttering ................................. 118

5.1.2 Stability of internal models ......................................................... 120

5.1.3 Sequence length and sensorimotor integration ............................... 122

5.2 The role of aberrant inhibition in stuttering ............................................. 123

5.2.1 Higher inhibitory levels during sensorimotor preparation in severe

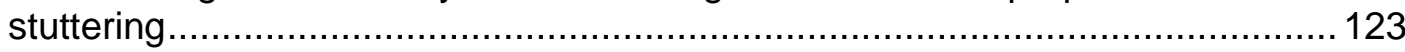

5.2.2 Influence of network inhibition on the retrieval of internal models......... 125

5.2.3 Testing the influence of right-hemispheric inhibition........................... 125

5.3 Conclusion and Outlook ....................................................................... 127

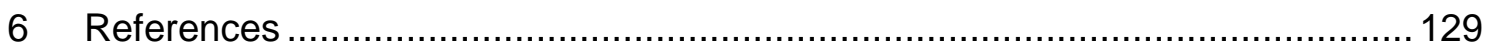


7 Appendices.

181

8 Declaration of Contribution 


\section{List of Abbreviations}

$A D$

axial diffusivity

AWS

adults with developmental stuttering

BDI

Beck Depression Inventory

BOLD blood-oxygenation-level dependent

CNS central nervous system

CWS children with developmental stuttering

DIVA Directions Into Velocities of Articulators $\mathrm{dMRI}$ diffusion-weighted magnetic resonance imaging

DTI diffusion tensor imaging

EEG electroencephalography

EMG electromyography

EPI echo planar imaging

ERP event related potential

EXPLAN

Execution and Planning model

FA fractional anisotropy

FAT frontal aslant tract

FC fluent controls

FDR false discovery rate

fMRI functional magnetic resonance imaging

FoV field of view

GODIVA Gradient Order Directions Into Velocities of Articulators $\mathrm{HC}$ healthy controls

HF high-frequency

$\mathrm{Hz}$ Hertz 
ICD international classification of diseases

IFG inferior frontal gyrus

IPC Index of Phonetic Complexity

LF low-frequency

LMC laryngeal motor cortex

MD mean diffusivity

MRI magnetic resonance imaging $\mathrm{ms}$ millisecond

OASES Overall Assessment of the Speaker's Experience of Stuttering PDS-....persons with developmental stuttering not taking part in any stuttering intervention PDS+ persons with developmental stuttering who participated in the stuttering intervention pSTG posterior superior temporal gyrus

$\mathrm{RD}$ radial diffusivity

$\mathrm{ROI}$ region of interest rs-fMRI resting-state functional magnetic resonance imaging $\sec$ second

SLF superior longitudinal fasciculus

SLRT Salzburger Lese- und Rechtschreibtest

SMA supplementary motor area

SMG supramarginal gyrus

SSI Stuttering Severity Index

$\mathrm{T} 1$ pre-intervention T2 post-intervention TE echo time $\mathrm{TI}$ inversion time TMS transcranial magnetic stimulation TR repetition time 
Abbreviations in Figure 1 and Figure 12 are specified in the respective legends below the figures. 


\section{List of Figures}

Figure 1. The Directions Into Velocities of Articulators model and its neural correlates. ... 4

Figure 2. Study 1: Cue-target paradigm showing a trial of the overt condition. .34

Figure 3. Study 1: Deleted face and neck electrodes of the EEG system. .38

Figure 4. Study 1: Reduced alpha and beta power during overt speech intention. 42

Figure 5. Study 1: Descriptive alpha and low beta power during speech intention. 43

Figure 6. Study 1: Influence of stuttering severity on low beta power during overt speech intention .44

Figure 7. Study 2: Workflow of pseudoword selection. .65

Figure 8. Study 2: Data for each factor contributing to stuttering events over all syllable positions. .73

Figure 9. Study 2: Data for each factor contributing to stuttered events in initial syllable position. 75

Figure 10. Study 2: Data for each factor contributing to errors overall syllable positions. 77 Figure 11. Study 2: Data for each factor contributing to errors in initial syllable position..79 Figure 12. Study 3: The four semi-discrete functional networks for the ROI-to-ROI resting state fMRI analysis. 98

Figure 13. Study 3: Intensive computer-assisted intervention reduced stuttering and strengthened IFG-to-LMC rs-connectivity within the sensorimotor integration network...105 Figure 14. Study 3: No intervention-related white matter changes in three speech motor tracts. 107

Figure 15. Study 3: Brain behavior relationship changed with intervention 109 


\section{List of Tables}

Table 1. Overview of paper selection on brain oscillations.......................................25

Table 2. Study 1: Demographic information of participants .......................................

Table 3. Study 1: No group differences on discarded EEG data ..................................38

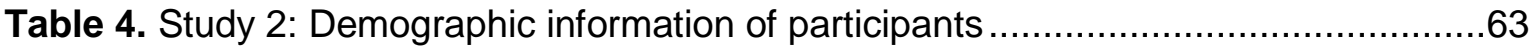

Table 5. Study 2: Linguistic factors of pseudowords and initial syllables.......................66

Table 6. Study 2: Descriptive statistics of stuttered events over all syllable positions in AWS.

Table 7. Study 2: Descriptive statistics of stuttered events in initial syllable positions in AWS.

Table 8. Study 2: Descriptive statistics of errors over all syllable positions per group. .....76

Table 9. Study 2: Descriptive statistics of errors in initial syllable positions per group......78

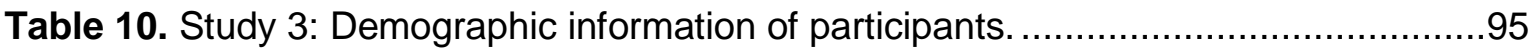

Table 11. Study 3: Brain hubs of speech-related sensorimotor integration. ....................99

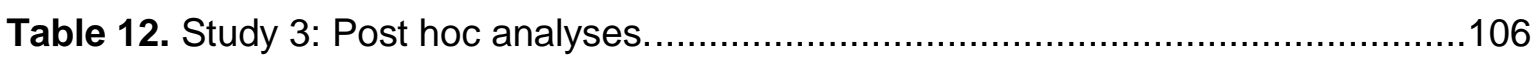




\section{List of Tables in Appendices}

Table A-1. Demographic information per participant. 181

Table A-2. Pre-assessment scores per participant 182

Table B-1. Demographic information per participant 184

Table B-2. Pre-assessment scores per participant. 185

Table B-3. List of pseudowords. 187

Table B-4. List of intitial syllables 196

Table B-5. Likelihood ratio tests of additional factors. 199

Table B-6. Minimum and maximum estimates derived from model stability analysis of stuttering events 200

Table B-7. Minimum and maximum estimates of model stability analysis of response accuracy

Table B-8. Mean of stuttered events and response errors in initial syllable position per participant. 202

Table C-1: Demographic information per participant. 207

Table C-2: Total scores of SSI and OASES at T1 and T2 per participant. 209

Table C-3. Brain hubs of speech (motor) planning 211

Table C-4. Brain hubs of inhibition 211

Table C-5. Brain hubs of articulatory convergence. 212

Table C-6. Summary of changes in behavioral outcome measures. 212

Table C-7. Posthoc comparisons on results of global ANCOVA differentiating the age effect between groups.

Table C-8. Group averages and test statistics of one-sample paired t-tests for the change of diffusion parameters in PDS+. 214

Table C-9. Group averages and test statistics of one-sample paired t-tests for the change of diffusion parameters in PDS215 
Table C-10. Group averages and test statistics of one-sample paired t-tests for the change of diffusion parameters in $\mathrm{HC}$ 216

\section{List of Figures in Appendices}

Figure C-1. Probability maps, waypoint and exclusion masks for the segmentation of the tract connecting LMC and IFG.

Figure C-2. Results of the global ROI-to-ROI resting-state connectivity analysis.

Figure C-3. Association between left IFG to left SMG connectivity change from T1 to T2 and age differentiated by group. 218 


\section{Summary}

Stuttering leads to involuntary disruptions of the speech flow. Stuttering is thought to emerge due to impaired speech planning, initiation, sensorimotor integration or inhibition of speech segments. Previous research has shown that adults with developmental stuttering (AWS) show aberrant modulation of neuronal oscillations during speech motor preparation, supporting theories of impaired sensorimotor integration or inhibition. However, neuronal oscillations are also modulated by linguistic factors.

To test these alternative explanations, experiment $1 \mathrm{a}$ in chapter 2 used a cue-target selfinitiated reading paradigm to distinguish sensorimotor preparation during speech intention from language processing in AWS. The results were fourfold: (1) both AWS and fluent controls (FC) showed sensorimotor preparation prior to overt compared with covert reading; (2) sensorimotor preparation of AWS did not differ from FC; (3) sensorimotor preparation in AWS prior to stuttered speech was similar to that prior to fluent speech; (4) stuttering severity was associated positively with beta $(15-25 \mathrm{~Hz})$ power increase. The results indicate that AWS with more severe stuttering show stronger inhibition during speech intention.

One parameter that could be influenced by higher or lower inhibitory levels in sensorimotor regions is the occurrence frequency of a movement. Experiment $1 \mathrm{~b}$ in chapter 3 tested the effect of syllable frequency on stuttering probability during the cue-target self-initiated reading paradigm of 300 pseudowords. The influence of syllable frequency on response accuracy in AWS and FC served as control conditions. Remarkably, only pseudoword length but not syllable frequency influenced stuttering probability of the initial syllable in AWS. Further, the significant group by frequency interaction showed that AWS made more response errors on high-frequency than on low-frequency initial syllables, whereas FC showed the expected pattern of fewer response errors on high-frequency compared with low-frequency initial syllables. Last, a robust word length effect on response accuracy in both AWS and FC was observed. The results favor the account of impaired speech initiation 
in stuttering emergence, which can be aggravated by reduced sensorimotor integration. Another approach to investigate causal mechanisms in stuttering is to examine the effect of successful stuttering interventions. Chapter 4 presents a magnetic resonance imaging study that investigated intervention-induced, long-term functional and structural changes in four semi-discrete networks of speech planning, initiation (i.e. articulatory convergence), sensorimotor integration and inhibition. The long-term stuttering intervention under study comprised the acquisition of a speech technique that softens phonatory and articulatory movements. The successful stuttering intervention led to increased functional connectivity between the left inferior frontal gyrus and the left laryngeal motor cortex (command-toexecution pathway) and between the left inferior frontal gyrus and the right superior temporal gyrus (auditory-to-motor pathway) within the sensorimotor integration network. Moreover, the intervention-induced decrease of stuttering severity was associated with stronger white matter connectivity of the left superior longitudinal fasciculus, whereas decreased suffering from stuttering post-intervention correlated with lower white matter integrity of the right frontal aslant tract. Together, these results suggest that speech motor learning was supported by structural and functional connectivity of the sensorimotor integration and inhibitory control network.

Overall, the results of the three experiments indicate that sensorimotor integration, speech initiation and inhibition jointly contribute to stuttering. This supports the notion of a systemlevel speech fluency disorder. 


\section{Zusammenfassung}

Originäres neurogenes nicht-syndromales Stottern führt zu unwillkürlichen Unterbrechungen des Sprechflusses. Als neurophysiologische Ursachen werden eine Beeinträchtigung der Sprechplanung, der Sprechinitiierung, der sensomotorischen Integration oder der Inhibition von Sprecheinheiten diskutiert. Bisherige Forschungsergebnisse zeigen das Erwachsene mit originärem neurogenen nichtsyndromalen Stottern eine veränderte Modulation von neuronalen Oszillationen während der Sprechvorbereitung zeigen. Diese Ergebnisse unterstützen Theorien die eine Dysfunktion der sensomotorischen Integration oder der Inhibition für die Entstehung von Stotterereignissen annehmen. Bisherige Studien zu neuronalen Oszillationen und Stottern nutzten jedoch linguistische Stimuli, welche die gemessenen Oszillationen beeinflussen können.

Um unterschiedliche Theorien zu Stottern zu untersuchen, wurde in Experiment 1a in Kapitel 2 eine Hinweis-Ziel-Aufgabe mit anschließendem selbst-initiiertem Lesen durchgeführt. Das verwendete Design ermöglichte so eine Differenzierung von neuronalen Prozessen der sensomotorischen Vorbereitung und der Sprachverarbeitung. Vier Ergebnisse konnten dabei identifiziert werden: (1) sowohl stotternde Erwachsene als auch flüssigsprechende Kontrollprobanden zeigten im Vergleich zu leisem Lesen eine verstärkte sensomotorische Vorbereitung auf das laute Lesen; (2) beide Gruppen unterschieden sich nicht in ihrer sensomotorischen Vorbereitung; (3) stotternde Erwachsene zeigten eine ähnliche sensomotorische Vorbereitung vor flüssigem und gestottertem Lesen; (4) mit steigender Stotterschwere erhöhte sich auch die Betaaktivität $(15-25 \mathrm{~Hz})$. Diese Ergebnisse sprechen für eine erhöhte Inhibition bei schwer stotternden Erwachsenen, die während der Erwartungsphase vor dem Sprechen auftritt.

Ein Faktor, der durch unterschiedliche Inhibitionsgrade von sensomotorischen Arealen beeinflusst werden könnte, ist die Ausführungshäufigkeit einer Bewegung. Experiment 1b 
in Kapitel 3 überprüfte daher den Einfluss der Auftretenshäufigkeit von Silben, d.h. der Silbenfrequenz, auf die Auftretenswahrscheinlichkeit von Stotterereignissen während der Hinweis-Ziel Aufgabe mit selbst-initiierten Lesen von insgesamt 300 Pseudowörtern. Als Kontrollbedingung wurde der Einfluss der Silbenfrequenz auf die Genauigkeit der Aussprache von stotternden Erwachsenen und flüssigsprechenden Kontrollprobanden untersucht. Bemerkenswert war, dass zwar die Länge eines Pseudowortes, nicht aber die Silbenfrequenz, die Auftretenswahrscheinlichkeit von Stotterereignissen in der Initialsilbe bei stotternden Erwachsenen beeinflusste. Zudem zeigte eine statistisch signifikante Interaktion zwischen den Probandengruppen und der Silbenfrequenz, dass bei stotternden Erwachsenen mehr Aussprachefehler bei Initialsilben mit hoher Silbenfrequenz als bei niedriger Silbenfrequenz auftraten. Flüssigsprechende Kontrollprobanden hingegen zeigten das erwartete Verhältnis einer geringeren Aussprachefehleranzahl bei hoher Silbenfrequenz im Vergleich zu Silben mit niedriger Silbenfrequenz auf. Weiterhin konnte ein robuster Längeneffekt hinsichtlich der Genauigkeit der Aussprache in beiden Probandengruppen beobachtet werden. Diese Ergebnisse unterstützen die Theorie der beeinträchtigten Sprachinitiierung bei Stotternden. Außerdem zeigen die Ergebnisse, dass diese Beeinträchtigung durch eine geschwächte sensomotorische Integration verstärkt werden kann.

Die Untersuchung der Gehirnaktivität nach einer erfolgreichen Stottertherapie ist eine weitere Möglichkeit um der Ursache des Stotterns näher zu kommen. Daher wird in Kapitel 4 eine Studie vorgestellt, die mithilfe der Magnet-Resonanz-Tomographie funktionelle und strukturelle Veränderungen in vier neuronalen Netzwerken des Sprechens nach einer Langzeit-Stottertherapie untersucht. Bei den vier Netzwerken, handelt es sich um abgegrenzte Areale, die jeweils für die Sprechplanung, die Sprechinitiierung, die sensomotorische Integration und die Inhibition von Relevanz sind. Die hier untersuchte Stottertherapie vermittelte eine neue Sprechweise, der weiche Stimmeinsätze und artikulatorische Bewegungen zugrunde liegen. Diese Studie zeigte, dass eine erfolgreiche 
Stottertherapie zu einer erhöhten Konnektivität von der Kommando-Ausführung Leitungsbahn (linker Gyrus inferior frontalis zu linker Repräsentation des Larynx im Motorkortex) und der auditiven-motorischen Leitungsbahn (linker Gyrus inferior frontalis zu rechtem Gyrus temporalis superior) innerhalb des neuronalen Netzwerkes der sensomotorischen Integration führte. Zusätzlich zeigte sich eine negative Korrelation die Stotterschwere nach der Therapie mit der weißen Substanz des linken Fasciculus longitudinalis superior. Im Gegensatz dazu korrelierte die Bewertung der eigenen Erfahrung mit dem Stottern positiv mit der weißen Substanz des rechten frontalen Aslant Traktes. Damit ergibt sich, dass das sprechmotorische Lernen durch die funktionelle und strukturelle Konnektivität innerhalb des sensomotorischen und des inhibitorischen Netzwerkes unterstützt wird.

Insgesamt, deuten die drei Studien dieser Dissertationsarbeit darauf hin, dass sowohl Beeinträchtigungen der sensomotorischen Integration und der Sprachinitiierung als auch ein erhöhtes Inhibitionslevel gemeinsam zu dem Auftreten von Stottern beitragen. Dies entspricht dem derzeitigen Vorschlag, Stottern als eine Systemstörung zu betrachten. 


\section{General Introduction}

Stuttering is a speech fluency disorder that is primarily recognized by the involuntary disruptions of the speech flow. Such disruptions can be sound and syllable repetitions (s-ssound), sound prolongations (sssound) or speech blocks (--speech) (Wingate, 1964). These speech dysfluencies can be accompanied by face and limb movements, such as grimacing, nodding or hand tapping, which are learned struggle behaviours (Bloodstein \& Ratner, 2008). To avoid stuttering, strategies such as word substitutions or sentence reordering are applied (Natke \& Kohmäscher, 2020). In addition, stuttering puts the affected person at a higher risk of bullying and social discrimination (e.g. Blood \& Blood, 2004; Davis et al., 2002).

Persistent stuttering can lead to limitations of functional communication and a decline the quality of life (e.g. Beilby et al., 2012; Blumgart et al., 2012; Craig et al., 2009). Several intervention methods exist for reducing the symptoms of stuttering (for reviews see Baxter et al., 2016; Bothe, Davidow, Bramlett, \& Ingham, 2006; Nye et al., 2013). However, relapse after conventional interventions is common (Craig, 1998), and none of these interventions can actually heal the disorder.

A better understanding of the causative mechanisms of stuttering could lead to improvements in the available interventions or even new ones. In addition, as research in aphasia has greatly contributed to the understanding of language processing, more closely examining stuttering can lead to an improved understanding of the neurological, linguistic and sensorimotor mechanisms involved in speech fluency (see Ziegler (2009) for a similar account on apraxia of speech).

This thesis contributes to answering the question about the underlying mechanisms responsible for stuttering by combining different perspectives and methods. In Chapter 1, I present the process of speech production in fluent speakers, the standard medical background of stuttering and four current theories on its causation as well as the goals of 
the thesis. In Chapter 2, I distinguish between the inherent processes of speech network preparation in adults with developmental stuttering (AWS) and those of language processing. This is achieved by examining neuronal oscillations during speech intention, a cognitive state prior to any specific language planning and movement preparation. Chapter 3 focuses on the final step of language planning before articulation by examining the influence of syllable frequency on stuttering probability. Chapter 4 presents the neurophysiological change in connectivity that was the result of a successful one-year stuttering intervention on a network-based account. In Chapter 5, I discuss the results of my three studies within the context of the presented theories.

\subsection{Speech production in fluent speakers}

Before introducing the topic of stuttering, it is important to know how speech production is processed in fluent speakers. Phonetic encoding and its neuronal correlates are presented here in depth, as this thesis focuses on sublexical speech processes in stuttering.

Speech production encompasses the transformation of conceptual thoughts into the articulation of coupled sounds. The speech production model of Levelt et al. (1999) suggests a multistage process. In this model, thoughts activate lexical concepts, which in turn lead to the retrieval of words that are marked to fit the syntactic context. After the retrieval of a word, phonological encoding begins. In phonological encoding the phonemic segments of the word are retrieved, syllabified and mapped onto metrical frames that mark for example stress patterns. Abstract phonological words are the output of phonological encoding. In phonetic encoding abstract phonological words are translated into articulatory plans by computing their gestural score. The gestural score is an abstract representation of the articulatory gestures, i.e. context dependent adaptations are performed during articulation. Levelt et al. (1999) suggested that speakers retrieve highly overlearned gestural patterns, high-frequency phonetic syllables, from a repository called mental syllabary. 
The Directions Into Velocities of Articulators (DIVA) neurocomputational model of speech production (Guenther et al., 2006; Guenther, 2016) starts with the mental syllabary of Levelt's model, referred to here as speech sound map, and describes phonetic encoding and articulatory processes. Specifically, the DIVA model details the sensorimotor interaction required for speech motor control during speech production (for neural correlates see Figure 1). The DIVA model encompasses the feedforward and the feedback control systems. Both systems converge at the speech sound map and at the articulator map, which controls the articulatory musculature. Contrary to Levelt's mental syllabary, the speech sound map includes frequently spoken (multisyllabic) words, syllables, and single phonemes. The speech sound map encodes the necessary motor, auditory and somatosensory programs of each speech sound. These programs are defined as sets of neurons and their axonal projections that generate a learned set of articulator movements (in the case of a motor program) or define the expected sensory signal.

Projections from the speech sound map are controlled by subcortical loops. In the feedforward control system, the cortico-basal ganglia loop controls the initiation of the next speech sound and the inhibition of the preceding speech sound (left loop in Figure 1). To enable the correct sequencing of speech sounds, the cognitive and sensorimotor context are identified within this loop. When a speech sound is activated via the cortico-basal ganglia loop, the encoding of the motor and sensory programs is started. The feedback control system encompasses the auditory and the somatosensory subsystem. Both systems detect and correct differences between their respective sensory programs and the current sensory state. When a deviation, i.e. an error, is identified, sensory error maps activate the feedback control map, which in turn corrects the motor command in the articulator map. 
Figure 1. The Directions Into Velocities of Articulators model and its neural correlates.

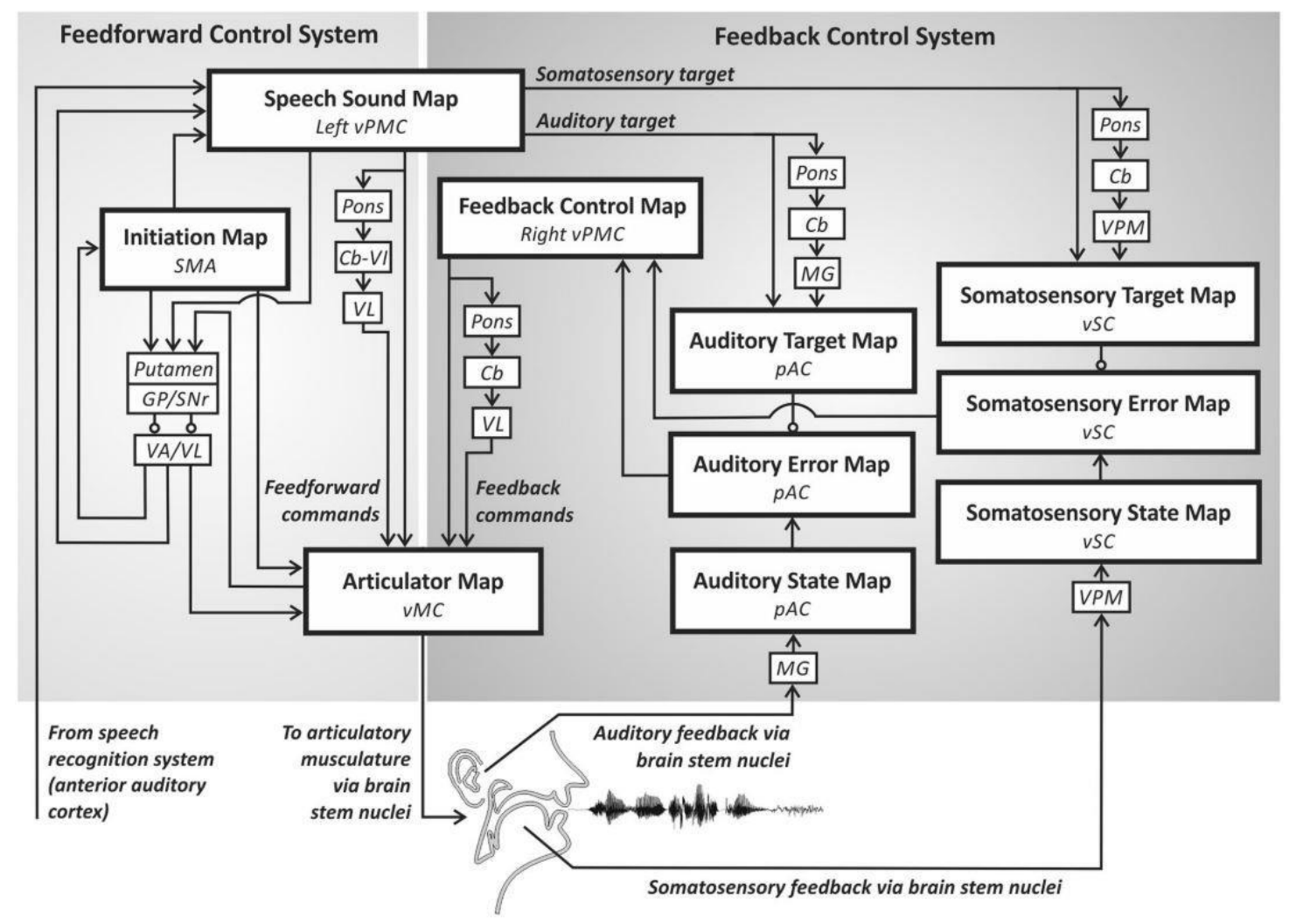

Note. Arrows represent excitatory projections. Lines with circles represent inhibitory projections. Boxes encompass computational model nodes and their correlating brain region. Cb, cerebellum; $\mathrm{Cb}-\mathrm{VI}$, cerebellum lobule $\mathrm{VI}$; GP, globus pallidus; MG, medial geniculate nucleus of the thalamus; pAC, posterior auditory cortex; SMA, supplementary motor area; SNr, substantia nigra pars reticula; VA, ventral anterior nucleus of the thalamus; VL, ventral lateral nucleus of the thalamus; VMC, ventral motor cortex; VPM, ventral posterior medial nucleus of the thalamus; vPMC, ventral premotor cortex; vSC, ventral somatosensory cortex. Modified from figure 4, p.8 (Kearney \& Guenther, 2019).

\subsection{Medical background of stuttering}

\subsubsection{Definition}

The $10^{\text {th }}$ version of the international classification of diseases (ICD), which is currently the official version in Germany, classifies stuttering as a "behavioral or emotional disorder with its onset usually occurring in childhood and adolescence". Stuttering is further defined by "[...] frequent repetition or prolongation of sounds or syllables or words, or by frequent hesitations or pauses that disrupt the rhythmic flow of speech. [...]" (F98.5, ICD-10, World Health Organization, 2011). Although this definition describes the core symptoms of 
stuttering, it excludes acquired stuttering in adults and accompanying symptoms of this speech fluency disorder. In the revision of the ICD, the World Health Organization now differentiates between persistent developmental stuttering (6A01), which is classified as a developmental speech fluency disorder, and adult onset stuttering (MA81) that is classified as speech disfluency (ICD-11, World Health Organization, 2019). The classification and symptomatology of childhood and adult onset stuttering disorders is in line with the German clinical practice guideline in speech fluency disorders (Neumann, Euler, Bosshardt, Cook, Sandrieser, Sommer, Thum et al., 2017). The guideline even differentiates between four types of stuttering. Adult onset acquired stuttering can have a neurogenic or psychogenic cause. On the other hand, persistent developmental stuttering is either referred to as idiopathic ("originary") neurogenic non-syndromal stuttering, when it emerges without discernible cause, or as idiopathic neurogenic syndromal stuttering, when stuttering cooccurs with a syndrome (e.g. Down Syndrome). In this thesis, I will use the terms (persistent) "developmental stuttering" or simply "stuttering" to refer to idiopathic neurogenic non-syndromal stuttering.

\subsubsection{Epidemiology}

In $95 \%$ of children with developmental stuttering (CWS), speech disfluencies emerge between the age of 2 and 4 years (Yairi \& Ambrose, 2013). On average children begin to stutter with 33 months, and for most children the risk for stuttering onset is over by age 5 (Yairi \& Ambrose, 2013). Thus, stuttering onset often co-occurs with an expanding vocabulary and the emergence of two- and three-word sentences (Natke \& Kohmäscher, 2020). The lifetime prevalence of stuttering has been estimated to be $1 \%$ (Bloodstein \& Ratner, 2008). However, if the entire age range, i.e. from 2 to 99 years, is evaluated one finds a lower overall prevalence of $0.72 \%$ (Craig et al., 2002). The childhood incidence of stuttering is approximately $8 \%$ as recent studies, employing professional case verification, have found (e.g. Reilly et al., 2009; and for a review: Yairi \& Ambrose, 2013). Developmental 
stuttering has a high rate of natural recovery, which can range from $71 \%$ to $94 \%$ (e.g. Månsson, 2000; Dworzynski et al., 2007). The sex ratio of men to women increases with increasing age. At onset the ratio is $3: 1$ and increases during adulthood to a ratio of $4: 1$ or 5:1 (Bloodstein \& Ratner, 2008; Neumann, Euler, Bosshardt, Cook, Sandrieser, \& Sommer, 2017; Yairi \& Ambrose, 2013). Epidemiological data suggest six risk factors for persistent developmental stuttering: a family history of (persistent) developmental stuttering, male sex, persistence of stuttering symptoms for more than six months, no reduction in stuttering severity within the initial 7-12 months, and an age of three years or older at the onset of stuttering (Neumann, Euler, Bosshardt, Cook, Sandrieser, Sommer, Thum et al., 2017).

\subsubsection{Etiology}

Genetic, neurological, and behavioral studies have been conducted to determine the causal factors for stuttering. Currently, developmental stuttering is seen as a multifactorial polygenic and neurogenic disorder, which can comprise multiple subtypes of distinct etiologies (Bloodstein \& Ratner, 2008; Neumann, Euler, Bosshardt, Cook, Sandrieser, Sommer, Thum et al., 2017). The following three paragraphs summarize the genetic and neurological findings associated with stuttering.

Since the 1930, the concept of stuttering as a hereditary disorder has motivated studies of familial incidence, concordance in di- and monozygotic twins, family aggregation and genes (Kraft \& Yairi, 2012). The first three study types investigated the distribution and relationships of persons with stuttering within families. Twin studies detected strong evidence for a genetic component ranging from an estimated 20 to $80 \%$ estimated heritability for stuttering (Kraft \& Yairi, 2012). Genetic studies sought to identify genes that contribute to stuttering. A number of genes have been shown to be linked with stuttering (for a comprehensive review see Frigerio-Domingues \& Drayna, 2017). Important in genetic studies is the reproducibility of results in unrelated cohorts in distinct ethnic groups. Mutations in four genes, GNPTAB on chromosome 12, GNPTG and NAGPA on 
chromosome 16p, and AP4E1 on chromosome 15, have been found repeatedly in families and in unrelated persons with stuttering spread over several countries (Raza et al., 2015; Raza et al., 2016). However, these mutations may only account for approximately $12-20 \%$ of unrelated persons with stuttering, leaving most stuttering cases unexplained (FrigerioDomingues \& Drayna, 2017).

Similar to the idea of inheritance, also the focus on brain abnormalities in stuttering started with the early $20^{\text {th }}$ century (for a review see Alm, 2005). However, the number of neurological studies investigating stuttering significantly increased only with the introduction of noninvasive neuroimaging methods, such as for example magnetic functional resonance imaging (fMRI) (for a review see Etchell et al., 2018). Following the publication of metaanalyses chronologically, the results regarding aberrant brain activity in stuttering are presented prior to those on brain structure. The meta-analyses revealed a greater activation in the right inferior frontal gyrus (IFG) and cerebellum, and a reduced activation in left auditory areas in AWS than in fluent controls (FC) (Belyk et al., 2015; Brown et al., 2005; Budde et al., 2014). To specify these results trait and state stuttering were compared. Trait stuttering compares two populations i.e. affected adults and healthy controls (AWS vs. FC) during fluent speech. State stuttering, on the other hand, is the comparison of the two speech patterns in the same population, stuttered vs. fluent, in affected adults (AWS). Trait stuttering is accompanied by an increased activation of the right IFG and pre supplementary motor area, and by a decreased activity in the left auditory cortex (Belyk et al., 2015; Budde et al., 2014). In addition, decreased activity in the left red nucleus (Budde et al., 2014), the left laryngeal motor cortex (LMC) and the left cerebellar vermis (Belyk et al., 2015) have been reported for trait stuttering. On the other hand, state stuttering revealed increased activity during stuttered speech in the left cerebellar vermis and bilateral supplementary motor area (SMA) (Belyk et al., 2015; Budde et al., 2014), and in the right LMC and lip motor cortex (Belyk et al., 2015). Further state stuttering showed decreased activity in right auditory areas (Belyk et al., 2015; Budde et al., 2014). 
One meta-analysis of diffusion tensor imaging (DTI) data gave robust evidence of aberrant white matter in AWS aged 14 to 52 years compared with FC (Neef et al., 2015). DTI studies examine white matter connectivity by measuring the directionality of water molecule mobility, i.e. fractional anisotropy (FA). The meta-analysis showed that the FA was reduced in the left dorsal language stream including fibers of the superior longitudinal fasciculus (SLF) and the arcuate fasciculus, as well as in the interhemispheric connections in the posterior midbody of the corpus callosum between sensorimotor cortices (Neef et al., 2015). The left dorsal language streams connects frontal, parietal and temporal language related regions, including the IFG, LMC and auditory areas (Saur et al., 2008). Although none of the meta-analyses included children, several studies have reported functional and structural differences in CWS (e.g. Beal et al., 2013; Chang et al., 2008; Chang et al., 2015). In addition, altered cortical thickness in AWS and CWS have also been reported (see the following sections). Taken together, neuroimaging data revealed that, at a group level, stuttering can be associated with structural and functional changes in the brain. These changes can indicate an impaired interaction within the language and speech production network, but their physiological foundation is not yet clear (Neef et al., 2015). Furthermore, the results of the meta-analyses must be regarded with caution as for example the group sizes in the primary studies were rather small, and analyses on state stuttering encompassed fluent and stuttered trials within their "stuttered speech" condition (Belyk et al., 2015; Neef et al., 2018).

\subsection{Theories on stuttering emergence}

Based on behavioural and neurological findings different theories have been proposed to account for the occurrence of stuttering symptoms. The following chapter introduces four currently discussed theories. I chose these theories as the following experiments are based on their postulates. There are, of course, also other theories and models in addition to those presented here (e.g. Namasivayam \& van Lieshout, 2011; Packman, 2012; Smith \& Weber, 
2017; Walden et al., 2012). The emergence of several, and partly interrelated, theories is explained by the fact that various processes contribute to speech production and stuttering (Neef et al., 2015). I added in my view central behavioural and neurological supporting findings to each. The chosen assignment of empirical findings is not a strict one, as other researchers may use the same findings in support of different theories.

\subsubsection{Sensorimotor Integration}

Behavioral findings

If the perceived auditory feedback of the speakers own voice is digitally altered (temporal or spectral shift), the speakers adapt their speech such that the speech output matches the altered auditory perception. This sensorimotor adaptation is based on left and right hemispheric feedback control processes (Floegel et al., 2020). During the application, such altered auditory feedback enhances speech fluency in AWS (e.g. Foundas et al., 2013; Pollard et al., 2009). In addition, AWS as opposed to FC exhibit a reduced motor adaptation, i.e. delayed start or reduced magnitude of motor adaption, to unexpected changes in auditory feedback (e.g. Cai et al., 2012; Cai, Beal et al., 2014; Loucks et al., 2012, but see Namasivayam et al., 2009 for null findings).

\section{Theory on impaired sensorimotor integration}

A deficit of sensorimotor integration in persons with developmental stuttering has been suggested by Max and colleagues (Max, 2004; Max, Guenther et al., 2004; for a similar model see Hickok et al., 2011). According to their model of motor control, speech execution is based on feedforward motor commands that are internally adjusted through a feedback control loop. The feedback control loop receives an expected sensory prediction from the feedforward motor command and compares this prediction with the actual sensory state. The feedback control can also adjust the motor command, such that movement execution will fit the expected sensory prediction. Max, Guenther et al. (2004) suggest that persons 
with developmental stuttering are unable to learn stable neural representations that map between motor and/or sensory signals. In their theory, these representations are called internal models. Internal models are comparable to the speech sounds in the DIVA model (see section 1.1). Alternatively, these internal models might be learned, but are insufficiently activated. The model presents two mechanisms, which could lead to stuttering. First, imprecise sensory predictive models could result in interfering feedback control. Due to a mismatch between the predicted sensory outcome and the actual sensory feedback, the feedforward command could be inadequately adjusted (Max \& Daliri, 2019). Second, unstable feedforward commands could result in incorrect movement preparation and execution, increasing the need for feedback based corrections (for neurocomputational modeling see Civier et al., 2010). In either case, stuttering is explained as a repeated attempt to produce a (variable) movement that fits the expected (impaired) sensory feedback. This proposal resembles that of Postma and Kolk (1993) (see section 1.3.2), except that sensorimotor instead of phonological errors are assumed here.

\section{Neurological findings supporting the theory}

For successful sensorimotor integration, brain regions of motor and sensory processing themselves as well as the structural connectivity between them must be intact. For speech production the ventral precentral gyrus and the motor cortex process feedforward commands, whereas the temporal lobe and the inferior parietal lobe are associated with auditory and somatosensory feedback processing, respectively (Guenther et al., 2006). The main white structure between sensorimotor regions is the SLF (Makris et al., 2005). Neuroimaging studies have reported aberrant grey matter in motor, auditory and somatosensory regions for both CWS and AWS (Beal et al., 2013; Chang et al., 2008; Garnett et al., 2018; Kell et al., 2009). In addition, trait stuttering is related to reduced white matter integrity between motor, sensory and temporo-parietal regions (Chang et al., 2008; Chang et al., 2015; Cykowski et al., 2010; Neef et al., 2015; Sommer et al., 2002; Watkins et al., 2008). It is thus not surprising that also the abnormal cerebral activation of auditory 
and motor regions have been reported for AWS, supporting suggestions of impaired feedforward and feedback modelling.

Several studies of electroencephalography (EEG) have examined sensorimotor integration in AWS by measuring auditory evoked potentials as the neurophysiological response to a sound (single tone or syllable). The clever move in these studies was to present the auditory stimulus during speech planning of overt speech, enabling the investigation of premovement sensory feedforward and feedback control. In FC the amplitude of the premovement auditory event-related potential is less strong, showing that sensory processing is altered during speech preparation (Max et al., 2008). Compared with FC, AWS do not show such a premovement auditory modulation (Daliri \& Max, 2015, 2018), suggesting that in them forward modelling of auditory input during speech planning is inefficient (Max \& Daliri, 2019). Further, $100 \mathrm{~ms}$ after auditory stimulus onset the current density over the auditory cortex correlated positively with stuttering severity (Mock et al., 2015). Aberrant sensorimotor integration has also been reported in studies investigating the modulation of neuronal oscillations in stuttering. Here, AWS show a stronger beta power decrease over motor regions during speech preparation (Mersov et al., 2016). This result is supported by another EEG sensor-level study that reports a stronger alpha power decrease in AWS, and correlations between stuttering severity and alpha and beta power (Mock et al., 2016). A stronger reduction of beta power may reflect an increased motor engagement as well as sensorimotor integration, which might be explained in the context of a higher inhibitory baseline level (see section 1.3.4). Contrary to the previous results, another research group reported reduced beta and alpha power over premotor regions in AWS during execution of overt speech (Jenson et al., 2018; Jenson et al., 2019), listening to white noise, and performing an auditory discrimination task (Saltuklaroglu et al., 2017). The authors interpreted these results as evidence for sensorimotor instability due to stronger motor reactivity, weak forward modeling and/or a reduced evaluation of sensory feedback. Taken together, the results show that the aberrant structural connectivity and the functional 
activity of AWS support the notion of impaired sensorimotor integration. However, due to the unclear implication of reduced auditory modulation and the diverging results concerning neuronal oscillations the functional role of sensorimotor integration in state stuttering is not yet clear.

\subsubsection{Planning}

Behavioral findings

Stuttering events do not occur randomly during speech production. For example, stuttering events mostly emerge at the beginning of utterances, words, and always at initial syllable positions (e.g., Richels et al., 2010; Wingate, 2002). In addition, the probability of stuttering increases, for example, according to the grammatical word class (content vs. function word), word length, and with phonetic complexity (e.g. Brown, 1945; Howell et al., 1999; Howell et al., 2006; Max et al., 2019). It seems, thus, natural to integrate language processes into theories of stuttering.

Theories on impaired linguistic planning

The covert repair hypothesis was developed on the assumption that speech errors are internally monitored during speech production and that this monitoring enables correction (Postma \& Kolk, 1993). The authors suggests that stuttering, i.e. blocks and repetitions, is the result of an increased error rate during the selection of phonemes, and a delayed covert repair of these selection errors (Postma \& Kolk, 1993). For counter arguments see, for example, Yaruss and Conture (1996).

The EXPLAN (Execution and Planning) model, on the other hand, accounts for speech disfluencies by considering the interaction between language planning and speech execution, excluding error monitoring and perception (Howell, 2004). The planning level in EXPLAN encompasses all levels of language production except articulation, i.e. semantic, syntactic, phonological and phonetic encoding. Contrary to that, the execution level, which 
is coupled to an internal timekeeper, receives and organizes the language plan for speech output. The internal timekeeper is a control mechanism that registers the time required for planning, and times the onset of execution in cases of misalignment between planning and execution levels. In EXPLAN, disfluencies occur when a language plan is not supplied to the execution system in time. More precisely, stuttering occurs when the speaker attempts to execute an incomplete plan. Howell (2004) suggests that this supply failure occurs either because of a prolonged planning time or an increased execution rate, i.e. speech rate, or both together. Planning can be delayed at any language level due to inherent properties of the planned segment, for example due to content words, the number of syllables or consonant strings (Howell, 2004).

\section{Neurological findings supporting the theory}

The EXPLAN model does not exactly define brain regions or connections, which could relate to delayed planning processes. Nevertheless, as Howell (2004) emphasizes that the number of syllables and consonant strings as possible stuttering inducing factors, I present neurological findings related to these two factors.

In fluent speakers, the IFG is a core region of language planning, which has been associated with semantic, syntactic and phonological sequencing (Price, 2010). In fact, among other nearby regions the left posterior IFG has been associated with syllable onset complexity (e.g. /bread/vs /red/) (Riecker et al., 2008). Further, the dorsal part of the IFG $[-56,8,20]$ together with the left inferior parietal lobe and the left SMA have been related to the planning of syllable sequences (Rong et al., 2018). The left IFG shows reduced grey matter volume in CWS and AWS (Beal et al., 2015; Cai et al., 2012; Chang et al., 2008; Kell et al., 2009). As described above, stuttering rate increases with sequence length. In the same region of the IFG $[-56,8,21]$ AWS show decreased activity during covert automatized speaking (months of the year) (Neef et al., 2016). Thus, the same part of the IFG that is related to the planning of sequences shows lower activity in trait stuttering. Close to the reported region, further studies have observed decreased activity in AWS compared with 
FC during sentence reading under normal and altered auditory feedback (Watkins et al., 2008). Remarkably, successful stuttering intervention was reported to normalize pretreatment hypoactivation of the IFG during generation of linguistic and emotional prosody (Neumann et al., 2018).

The left inferior parietal lobe is the second region associated with word length. Neef et al. (2016) also reported a reduced functional connectivity between the left posterior IFG and the left inferior parietal lobe in AWS. As the participants were performing covert speaking, the authors suggested that the reduced activity related to speech planning rather than to execution. Reduced white matter connectivity between the left IFG and the left inferior parietal lobe (e.g. Neef et al., 2015) in AWS supports this interpretation.

The third region associated with syllable sequencing is the left SMA. AWS have a reduced white matter connectivity of the left frontal aslant tract (FAT), which connects the IFG with the SMA (Kronfeld-Duenias et al., 2016b). Further, the stimulation of the left FAT during neurosurgery can induce stuttering (Kemerdere et al., 2016). As the SMA also plays a crucial role during the initiation of voluntary movement, further findings are presented in the following section. The same applies for the cerebellum, a brain region emphasized as internal timekeeper in the EXPLAN model. Thus, neuroimaging results support the idea of a delayed or impaired speech planning mechanism, which could be related to the processing of sequence complexity.

\subsubsection{Timing / Initiation}

\section{Behavioral findings}

The idea that stuttering could be a dysfunction of internal timing stems from observations that stuttering occurs during spontaneous speech, but speech fluency is immediately achieved when speaking to an external rhythm (e.g. metronome or singing) (Alm, 2004). However, studies comparing the abilities of AWS compared to FC to synchronize 
movements to a perceived rhythm yielded inconsistent results (group difference: Falk et al., 2015; Olander et al., 2010; no group difference: Hilger et al., 2016; Max \& Yudman, 2003).

\section{Theories on impaired timing}

Alm (2004) developed the dual premotor model to explain stuttering symptoms as disruptions of a sequential (speech) motor behavior. The dual premotor model describes a medial and a lateral pathway of motor timing. The medial pathway includes the basal ganglia, the SMA and the premotor cortex and is associated with the automatized execution of speech. The basal ganglia are believed to produce timing cues that enable the SMA to signal movement initiation. In this model, stuttering is induced by a dysfunction of the basal ganglia leading to an insufficient initiation of the planned or to an impaired inhibition of the previous speech segment. Speech fluency improves if speech timing is provided by the lateral premotor system. The lateral premotor system consists of the cerebellum and the lateral premotor cortex. According to Alm (2004) timing of the lateral premotor system is linked either to external stimuli (e.g. metronome) or to increased conscious attention (e.g. consciously slowed speech rate). The idea of a dysfunction within the left hemispheric cortico-basal ganglia-thalamo-cortical loop in stuttering has been adopted into a neurocomputational model (Civier et al., 2013). In this model, which is based on the DIVA model, stuttering was modeled by manipulating two parameters. First, impaired corticostriatal projections from the motor cortex through the basal ganglia induce stuttering by hampering the inhibition of the previous syllable, which leads to a delayed initiation of the next syllable. Second, an increase of the striatal dopamine level leads to an insufficient inhibition of competing incorrect motor programs, so that the initiation of the correct motor program is delayed.

Neurological findings supporting the theory

The contribution of the cortico-basal ganglia loop to speech fluency is supported by studies reporting stuttering emergence after lesions to the putamen or the SMA (Ackermann et al., 
1996; Tani \& Sakai, 2011; Theys et al., 2013). Cerebellar lesions can either lead to stuttering (Tani \& Sakai, 2010) or terminate stuttering (Primaßin, 2019). Although accompanied with side effects, pharmaceutical drugs that block the striatal dopamine receptors can reduce stuttering symptoms to a certain level (Bothe, Davidow, Bramlett, Franic, \& Ingham, 2006; Maguire et al., 2020). Last of all, several studies reported reduced white matter integrity, and aberrant functional activity and connectivity within the cortico-basal ganglia circuit in CWS and AWS (Chang et al., 2015; Chang \& Zhu, 2013; Lu, Chen et al., 2010; Lu, Peng et al., 2010; Metzger et al., 2018; Qiao et al., 2017). Detailed reviews of the role of the SMA and the cortico-basal ganglia loop in stuttering can be found in Busan (2020) and Chang and Guenther (2019). Stuttering interventions that lead to a decrease in stuttering severity reduce over activation of the basal ganglia and the cerebellum (Lu et al., 2012; Toyomura et al., 2015); possibly the overreliance on the external timing loop decreases while the activity within the internal timing loop normalizes. In addition, therapy induced changes in the basal ganglia and the cerebellum have been reported to negatively correlate with stuttering severity (Giraud et al., 2008; Lu et al., 2012).

Although behavioral studies report inconsistent findings, functional neuroimaging during timing tasks revealed that there is no correlation between timing-related networks and rhythm performance in CWS (Chang et al., 2016). Further, unlike FC, CWS showed an aberrant pattern of oscillatory modulation, i.e. a decrease (instead of an increase) of beta power prior to a timed auditory stimulus (Etchell et al., 2016). Thus, although behavioral results are inconsistent, CWS show deviant neural mechanisms of timing. However, it remains unclear how impaired timing of speech segments can explain inter- and intrasubject variability of stuttering occurrences.

\subsubsection{Inhibition}

Behavioral findings

A recent interest in temperamental characteristics in stuttering has led to interesting findings 
relative to behavioral and cognitive control mechanisms in persons with developmental stuttering. For example, a negative correlation between stuttering severity and effortful control, i.e. inhibiting a dominant response and executing a subdominant response, was reported for 98 children with developmental stuttering (Kraft et al., 2019). Further, studies examining, performance during movement inhibition tasks, e.g. Go/NoGo or Stop-Signal tasks, indicated deficient motor response inhibition abilities in AWS and CWS compared with FC (Eggers et al., 2013; Markett et al., 2016). However, other studies reported similar performances of participants with developmental stuttering and FC during response inhibition tasks (Eggers \& Jansson-Verkasalo, 2017; Piispala et al., 2016).

\section{Theory on impaired inhibition}

So far, the presented models on stuttering have focused on left-hemispheric language processes. Nevertheless, one trait marker of stuttering is the increased activity of the right IFG during fluent speech in AWS (see section 1.2.2). This trait marker has been mostly interpreted as a result of impeded left-hemispheric language and speech processing leading to an increased right-hemispheric involvement, and probably indicating a compensatory mechanism, which enhances speech fluency (Alm, 2004; Chang \& Guenther, 2019; Preibisch et al., 2003; Sommer et al., 2002). This suggestion was further supported by a decrease in right-hemispheric activity after successful stuttering intervention (Kell et al., 2009; Neumann et al., 2003). However, a recent study revealed that in AWS the time course of the blood-oxygenation-level dependent (BOLD) response differed between left and right dorsal IFG (Neef et al., 2016). More specifically, peak latencies of the BOLD responses in the right IFG were delayed and related to the offset of the task. Based on these observations, the authors suggested that within the network of inhibitory motor control the right IFG might be causally associated with stuttering. In addition to the weakened left hemispheric forward modelling, which elevates the conflict between several feedforward plans, an increased unspecific overactive response inhibition mechanism via the right IFGbasal ganglia may induce stuttering (Metzger et al., 2018; Neef et al., 2016). 
Neurological findings supporting the theory

The hypothesis of Neef et al. (2016) was further supported by another study by the same research group. Replicating the results, Neef and colleagues reported increased activity of the right IFG and the middle frontal gyrus in 31 AWS compared with 34 FC during imaginary speaking (Neef et al., 2018). Using fMRI-based tractography, the authors further showed that underneath the right hemispheric overactive regions, the right FAT and the right anterior thalamic radiation correlated positively with the stuttering severity of AWS. These results were held to confirm the hypothesis of an overactive inhibition in stuttering (Neef et al., 2018). In this study, AWS also showed increased activity of the frontal pole. However, the underlying part of the uncinate fasciculus correlated negatively with stuttering severity (for similar results in CWS see Chang et al., 2015). As the uncinate fasciculus connects the frontal pole with the superior temporal gyrus, this finding was interpreted as a compensatory mechanism that recruits additional regions of multisensory monitoring to enhance speech fluency (Neef et al., 2018). This result might explain the behavioral results of increased effortful control in children with less severe stuttering. In addition, support of the involvement of an inhibitory network in stuttering comes from studies investigating neuronal oscillations. Alpha and beta power are thought to reflect activating and inhibitory processes of the cognitive state (Jenkinson \& Brown, 2011; Klimesch, 2012). CWS showed a greater decrease in alpha power prior to a Go/NoGo task, which was interpreted as a reduced inhibition of the visual cortex, and sensory information suppression (Piispala et al., 2018). In addition, a greater beta power increase in AWS was observed over left and right motor regions after speech execution and before the next linguistic stimulus (Mersov et al., 2016). This result was interpreted as an increased inhibitory level in speech motor regions in AWS (Mersov et al., 2016). Finally, inhibitory repetitive transcranial magnetic stimulation (TMS) of the right anterior IFG pars triangularis led to increased fluency during reading and decreased fluency during speaking in eight AWS (Tezel-Bayraktaroglu et al., 2020). Taken together, recent studies suggest that inhibition may play a relevant role in state stuttering. 


\subsection{Objectives and structure of this dissertation}

The previous sections have shown that the cause of stuttering is still unknown and that different mechanisms during speech production may explain the emergence of stuttering symptoms. For all theories support can be found on a behavioural, neuroanatomical and neurophysiological level. Indeed, even individual studies reveal aberrant activation and functional connectivity in AWS within regions that are associated with speech planning, timing/initiation and execution (Lu, Chen et al., 2010; Qiao et al., 2017; Xuan et al., 2012). However, none of the theories can account for all the findings of trait and state stuttering. It is thus an open question, whether different mechanisms interact as suggested by Neef et al. (2016), or if the presented mechanisms can account for specific subtypes of stuttering (Chang \& Guenther, 2019). In my view, stuttering is a complex disorder encompassing several neuronal networks and/or cognitive processes and research can only contribute to the bigger picture of stuttering in small steps.

The aim of this dissertation was to further the knowledge of trait and state stuttering by examining different aspects of the four theories of stuttering presented above. To achieve this aim, I combined psycholinguistic and neurophysiological perspectives on stuttering and used several methods in two projects. I designed the first project to target the idea of insufficient activation of internal models as described in the theory of aberrant sensorimotor integration (Section 1.3.1) and to examine the final process of speech planning (Section 1.3.2) in stuttering. To investigate several of the propositions, I used a cue-target design for triggering self-initiated reading. This thesis focuses on the brain responses after the cue stimulus and the behavioral response after the target stimulus (Chapters 2 and 3); whereas the analyses of brain responses after the target stimulus and prior to self-initiated speech are not part of this thesis. The second project, presented in Chapter 4, examines the influence of stuttering intervention on the brain networks underlying the four theories. For the second project, I used resting-state fMRI data that had been collected during the dissertation project of Annika Primaßin (Primaßin, 2019). 
Thus, in Chapter 2, I examine the formation of the speech production network in AWS by recording neuronal oscillations in a speech intention task (brain response to the cue stimulus). During the formation of the speech production network cortical activation increases in sensorimotor regions relevant for speech production (Gehrig et al., 2012). This activity indicates sensorimotor preparation. The research question was whether trait and state stuttering related to aberrant sensorimotor activation of the speech production network prior to any linguistic processing.

In Chapter 3, I investigate the influence of syllable frequency on stuttering rate and response accuracy in a self-initiated pseudoword reading task. High-frequency syllables are known to reduce the speech planning time in fluent speakers (Cholin et al., 2006). Thus, the research question was whether high-frequency syllables, which represent more stable internal models and decrease planning time, induced less stuttering and more accuracy compared with low-frequency syllables.

In Chapter 4, I examine the influence of a one-year intense stuttering intervention on functional and structural connectivity within semi-discrete neuronal networks of speech planning, speech timing (in this study articulatory convergence), sensorimotor integration and speech inhibition. Studies showed that stuttering interventions improved abnormal activity and functional connectivity in regions contributing to different functional networks (see 1.3). However, it was still not clear how stuttering interventions influenced brain connectivity on a network-based level. The research question was whether successful stuttering intervention strengthened speech specific networks associated with stuttering causation.

In Chapter 5, I discuss the results of this thesis in a broader context and give perspective for future investigations. 


\section{Increased right frontal beta power during speech intention in severe stuttering}

Authors: Alexandra Korzeczek ${ }^{1}$, Iris Steinmann², Nicole E. Neef ${ }^{1}$, Walter Paulus ${ }^{1}$, and Martin Sommer ${ }^{1,3}$

\section{Affiliation:}

1'Department of Clinical Neurophysiology, University Medical Center Göttingen, Göttingen, Germany;

²Department of Cognitive Neurology, University Medical Center Göttingen, Göttingen, Germany;

${ }^{3}$ Department of Neurology, University Medical Center Göttingen, Göttingen, Germany.

will be submitted shortly

\section{Authors contributions:}

A.K., and M.S. conceived and designed the experiment; A.K. administered the project, performed the experiments, evaluated, analyzed, visualized and interpreted the data, wrote and revised the paper; I.S., and N.E.N. contributed to data analysis and interpretation of the results; W.P. contributed to the study design and provided the resources; all authors reviewed the manuscript. 
2. Increased right frontal beta power during speech intention in severe stuttering

\section{Abstract}

Objective: The neurophysiological correlates of the likely occurrence of a stuttering event are still unknown. This sensor-level EEG study investigated whether the transient formation, i.e. decrease in alpha and beta power, of the speech production network due to speech intention may lay open such neurophysiological marker. The modulation of alpha and beta power is associated with changes in the sensorimotor state indicating the maintenance or release from the current cognitive and/or sensorimotor state.

Methods: We used time-frequency analyses to study alpha $(8-13 \mathrm{~Hz})$, low beta $(15-25 \mathrm{~Hz})$ and high beta $(25-30 \mathrm{~Hz})$ power in 19 adults with developmental stuttering (AWS) and 19 fluent controls (FC) during speech intention. A cue-target reading paradigm separated preparatory activity of the speech production network during speech intention from language related processing.

Results: During overt compared to covert speech intention, alpha and low beta power was reduced in both groups. A decrease in alpha and beta power relative to baseline emerged however only over posterior EEG sensors covering parietal regions, possibly indicating that the intention to read prevailed over the intention to speak. Further, prior to fluent speech, stuttering severity was associated positively with a significant increase in low beta power in EEG sensors covering left parietal, central and right temporal regions. This association was less pronounced prior to stuttered speech. No other differences emerged.

Conclusion: Similar to fluent speakers, AWS show oscillatory activity modulations during the intention to speak overtly. However, in persons with more severe stuttering this task setup is overlaid with stronger beta power increase, possible implicating a stronger maintenance of the current sensorimotor state. 
2. Increased right frontal beta power during speech intention in severe stuttering

\section{Introduction}

Developmental stuttering is a speech fluency disorder (ICD-11, World Health Organization, 2019) comprising neurophysiological correlates within the speech production network (Watkins et al., 2008). Models of the speech production network allocate speech preparation to the left inferior frontal cortex, movement initiation to the supplementary motor area within a basal-ganglia-thalamo-cortical loop, and motor execution to the motor cortex (Bohland et al., 2010; Guenther \& Vladusich, 2012; Hickok, 2012; Kotz \& Schwartze, 2010). In adults with developmental stuttering (AWS), these regions show abnormal structural connectivity (Kronfeld-Duenias et al., 2016a; Neef et al., 2015; Sommer et al., 2002) and functional activity (Belyk et al., 2015; Budde et al., 2014) in trait and state designs, as revealed by MRI studies. Findings suggest a hyperdopaminergic level in the basal ganglia (Metzger et al., 2018; Watkins et al., 2008; Wu et al., 1997) and a dysregulation of inhibitory and excitatory activity during speech production (Alm, 2004; Civier et al., 2013). However, changes in the BOLD signal, as revealed in fMRI studies, cannot precisely explain whether the observed change results because of inhibitory or excitatory neuronal activation (Lauritzen et al., 2012; Waldvogel et al., 2000). These processes can be captured by neuronal oscillations (Jenkinson \& Brown, 2011; Klimesch, 2012).

Neuronal oscillations are measured with electroencephalography (EEG) or magnetoencephalography. Neural assemblies and single neurons reveal rhythmic, and thus oscillatory, spiking patterns over time. These are thought to enable information transfer between distinct neural networks (Buzsáki, 2006). Different frequency bands of neural oscillations can be distinguished and are associated with various cognitive processes (Buzsáki et al., 2013). For example, modulations of the alpha band, ranging between 8 and $13 \mathrm{~Hz}$, have been associated with successful visual perception (Romei et al., 2010; van Dijk et al., 2008), auditory processing (Hartmann et al., 2012; Lehtelä et al., 1997; Müller \& Weisz, 2012) and speech comprehension (Drijvers et al., 2018; Obleser \& Weisz, 2012). Neuronal oscillations can increase in their power, i.e. squared amplitude, relative to a 
2. Increased right frontal beta power during speech intention in severe stuttering

baseline, also termed event-related synchronization, as response to task demands or an event. Only two of these frequency bands, the alpha and beta (13 to $30 \mathrm{~Hz}$ ) band, have been shown to decrease in power, i.e. event-related desynchronization (Klimesch, 2012). Current theories on oscillatory power changes, suggest that cortical areas show an eventrelated power increase, reflecting inhibition of cognitive processes; whereas, the decrease in power indicates disinhibition and thus cortical activation (Jenkinson \& Brown, 2011; Jensen \& Mazaheri, 2010; Klimesch et al., 2007). Extending the proposal of disinhibition and inhibition, Engel and Fries (2010) suggest that modulations of beta power indicate the maintenance (increase in power), the change (decrease in power) or no change (unchanged) of the current motor, cognitive or perceptual set.

Beta power decrease over cortical sensorimotor areas relates to movement processing. Decreases in beta power are observed during and prior to movement execution (Alegre et al., 2006; Stancák \& Pfurtscheller, 1996; Zaepffel et al., 2013) and also during speech preparation (Grabner et al., 2007; Herman et al., 2013; Salmelin \& Sams, 2002). In addition to speech preparation and execution, language processes also induce beta power decrease (Weiss \& Mueller, 2012). Alpha and beta power modulations are also observed simultaneously, termed as mu-rhythm, during speech preparation and execution (Bowers et al., 2018; Gehrig et al., 2012; Jenson et al., 2014) and speech comprehension (Drijvers et al., 2018). Based on such findings, Jenson et al. (2019) developed a model of alpha and beta power modulation of speech processing, distinguishing cognitive and motor functions of both frequency bands. During speech motor preparation prior to speech onset, decreased power indicates the preparatory evaluation of sensory feedback (alpha) and forward modelling of the speech motor programs (beta). In addition, attentional processes prior to speech onset lead to an increase in alpha power supporting attentional allocation and a decrease in beta power supporting predictive coding. 
2. Increased right frontal beta power during speech intention in severe stuttering

Neuronal oscillations of speech preparation or execution in AWS have so far been studied in ten studies (Jenson et al., 2018; Jenson et al., 2019; Mersov et al., 2016; Mersov et al., 2017; Mock et al., 2016; Rastatter et al., 1998; Salmelin et al., 2000; Sengupta et al., 2016; Sengupta et al., 2017; Sengupta et al., 2019) (Table 1).

Table 1. Selection of papers on brain oscillations

\begin{tabular}{|c|c|c|c|c|c|c|c|}
\hline Study & Device & $\begin{array}{l}\text { Con- } \\
\text { trast }\end{array}$ & AWS & FC & Task & Stimuli & $\begin{array}{l}\text { Speech } \\
\text { process }\end{array}$ \\
\hline $\begin{array}{l}\text { Rastatter } \\
\text { et al., } 1998\end{array}$ & EEG & trait & 7 & 7 & $\begin{array}{l}\text { reading, } \\
\text { under } \\
\mathrm{N}-/ \mathrm{AAF}\end{array}$ & text passage & execution \\
\hline $\begin{array}{l}\text { Mock et } \\
\text { al., } 2016\end{array}$ & EEG & trait & 12 & 12 & $\begin{array}{l}\text { primed } \\
\text { picture } \\
\text { naming }\end{array}$ & $\begin{array}{l}\text { nouns (1 or } 2 \\
\text { syl.) }\end{array}$ & preparation \\
\hline $\begin{array}{l}\text { Jenson et } \\
\text { al., } 2018\end{array}$ & EEG & trait & 24 & 27 & reading & $\begin{array}{l}\text { sequences of } \\
\text { /ba and /da/ } \\
\text { ( } 2 \text { syl.), } \\
\text { and nouns ( } 3 \\
\text { syl.) }\end{array}$ & $\begin{array}{l}\text { preparation } \\
\text { and } \\
\text { execution }\end{array}$ \\
\hline $\begin{array}{l}\text { Jenson et } \\
\text { al., } 2019\end{array}$ & EEG & trait & 11 & 11 & $\begin{array}{l}\text { delayed } \\
\text { reading }\end{array}$ & $\begin{array}{l}\text { sequences of } \\
/ \mathrm{ba} /, / \mathrm{ma} /, \\
\text { /pa/, and /wa/ } \\
\text { ( } 2 \text { or } 4 \text { syl.) }\end{array}$ & execution \\
\hline $\begin{array}{l}\text { Sengupta } \\
\text { et al., } 2016\end{array}$ & EEG & trait & 8 & 8 & $\begin{array}{l}\text { speech } \\
\text { motor } \\
\text { adaption } \\
\text { task to } \\
\text { AAF }\end{array}$ & noun /head/ & execution \\
\hline $\begin{array}{l}\text { Sengupta } \\
\text { et al., } 2017\end{array}$ & EEG & state & 8 & & $\begin{array}{l}\text { delayed } \\
\text { reading }\end{array}$ & $\begin{array}{l}\text { nonwords ( } 2 \\
\text { to } 6 \text { syl.) }\end{array}$ & preparation \\
\hline $\begin{array}{l}\text { Sengupta } \\
\text { et al., } 2019\end{array}$ & EEG & trait & 8 & 8 & $\begin{array}{l}\text { delayed } \\
\text { reading }\end{array}$ & $\begin{array}{l}\text { nonwords ( } 2 \\
\text { to } 6 \text { syl.) }\end{array}$ & preparation \\
\hline $\begin{array}{l}\text { Salmelin et } \\
\text { al., } 2000\end{array}$ & MEG & trait & 9 & 10 & $\begin{array}{l}\text { delayed } \\
\text { reading }\end{array}$ & $\begin{array}{l}\text { words ( } 7 \text { to } 8 \\
\text { letters) }\end{array}$ & preparation \\
\hline
\end{tabular}


2. Increased right frontal beta power during speech intention in severe stuttering

\begin{tabular}{|c|c|c|c|c|c|c|c|}
\hline $\begin{array}{l}\text { Mersov et } \\
\text { al., } 2016\end{array}$ & MEG & trait & 12 & 12 & $\begin{array}{l}\text { delayed } \\
\text { reading }\end{array}$ & $\begin{array}{l}\text { word ( } 2 \text { syl.) } \\
\text { in carrier } \\
\text { phrase }\end{array}$ & $\begin{array}{l}\text { preparation } \\
\text { and } \\
\text { execution }\end{array}$ \\
\hline $\begin{array}{l}\text { Mersov et } \\
\text { al., } 2017\end{array}$ & MEG & state & 8 & - & $\begin{array}{l}\text { delayed } \\
\text { reading }\end{array}$ & $\begin{array}{l}\text { word ( } 2 \text { syl.) } \\
\text { in carrier } \\
\text { phrase }\end{array}$ & $\begin{array}{l}\text { preparation } \\
\text { and } \\
\text { execution }\end{array}$ \\
\hline
\end{tabular}

Note. Trait contrast: AWS vs. FC. State contrast: stuttered vs. fluent speech, $E E G$ = electroencephalography. $\mathrm{MEG}=$ magnetoencephalography. NAF = normal auditory feedback. AAF = altered auditory feedback. syl. = syllables

The first publication in the list showed that during a reading task with normal auditory feedback beta power was greater in AWS than in fluent controls (FC) (Rastatter et al., 1998). This finding was not replicated by later studies examining the reading of single words. Two studies reported a reduced decrease in beta and alpha power in AWS during fluent speech execution (Jenson et al., 2018; Jenson et al., 2019), whereas another study observed a stronger decrease in beta power in AWS than in FC (Mersov et al., 2016). Thus, findings relating to beta power modulation during speech execution have not been consistent and might have been due to different speech tasks (Table 1). Interestingly, after speech execution and prior to the appearance of the following word stimulus, Mersov et al. (2016) found a stronger beta power increase in AWS than in FC. In order to differentiate between speech preparation and speech execution seven studies used a cue-target task to induce delayed reading. The cue stimulus consisted of a linguistic stimulus and a second delayed "go"- stimulus requiring overt speech production (Table 1). Compared with FC, AWS showed a significantly stronger decrease in beta (Mersov et al., 2016) and alpha power (Mock et al., 2016) during speech preparation. In addition, Mock et al. (2016) reported a significant positive correlation between beta power decrease and stuttering severity. Taken together, the results suggest that AWS have an aberrant decrease in alpha and beta power. However, the interpretation of such aberrant modulations is not yet clear. In view of a reduced power decrease, a weakened sensorimotor feedforward modeling and evaluation of sensory feedback was suggested (Jenson et al., 2018; Jenson et al., 2019). Contrary to 
2. Increased right frontal beta power during speech intention in severe stuttering

this suggestion, the result of a stronger beta power decrease during speech preparation and execution with a stronger post movement beta power increase in AWS was interpreted as an impaired automatization of motor plans combined with stronger motor inhibitory baseline levels (Mersov et al., 2016).

Oscillatory differences between stuttered and fluent speech in AWS were examined in two studies. Whereas one study could not find significant differences in 12 AWS (Mersov et al., 2017), the other reported that in eight AWS speech preparation of fluent speech differed in alpha, beta and gamma power from speech preparation prior to stuttered speech (Sengupta et al., 2017). Further studies from this research group applying the same analysis method, i.e. linear classification, observed trait differences in four frequency bands, including alpha and beta power, during a sensorimotor adaption task and during speech preparation of difficult nonwords (Sengupta et al., 2016; Sengupta et al., 2017). However, interpretation of these results in comparison with the other studies is difficult as different analysis methods were used and the time-frequency data of groups or fluency states during speech preparation were not shown. Thus, speech preparation may differ between fluency states, however whether sensorimotor regions show a stronger inhibition or disinhibition or a weakened forward modelling of motor plans is unclear.

Most of these studies addressed speech motor preparation after the presentation of linguistic stimuli. One limitation of such designs is that linguistic computations influence speech motor preparation. For example, oscillatory power changes with regard to word frequency or sequence length during speech motor preparation (Grabner et al., 2007; Herman et al., 2013). Both of these factors are among the linguistic factors that can increase stuttering probability in AWS (for a detailed review see Wingate, 2002). Some suggest that AWS have a less stable speech motor system that breaks down, i.e. leads to stuttering, if additional cognitive load is put onto this system (Namasivayam \& van Lieshout, 2011; van Lieshout et al., 2004). Therefore, it would be desirable to investigate whether a core instability of the speech motor network exists (Neef et al., 2015; Rosenfield, 2013). One 
2. Increased right frontal beta power during speech intention in severe stuttering

possible way to disentangle such a core network from additional processes is to investigate the top-down process of task set-up, i.e. the intention to perform a task. Previous work has shown, that pre-task brain activity differs depending on the intended task (Sakai \& Passingham, 2006). For example, intending to communicate with a partner leads to stronger activation of the ventrolateral and ventromedial prefrontal cortex compared with speaking outside of a conversational context (e.g. calibrating the microphone) (Kuhlen et al., 2017). In the case of speech production, the intention to speak overtly already induces the leftlateralization of the speech production network in left sensory cortices, whereas a more bilateral network is seen for executive control of task rules (Kell et al., 2011; Keller \& Kell, 2016). In addition, further regions of the speech production network, including bilateral supplementary motor areas and bilateral inferior frontal gyri, were activated early during speech intention (Gehrig et al., 2012; Kell et al., 2011; Keller \& Kell, 2016). Activation of the speech production network during speech intention also manifested in beta and alpha power decrease over bilateral sensorimotor regions as well as left-lateralized alpha decrease over temporal regions (Gehrig et al., 2012). Thus, speech intention leads to oscillatory and hemodynamic changes in cortical regions that have been reported as structural and neurophysiological markers of developmental stuttering.

In this EEG study, we analyze neuronal oscillations that contribute to the neurodynamic setup of the speech production network, namely during speech intention prior to self-initiated speech. We define overt speech intention as the process of preparing to read subsequent stimuli overtly in contrast to covert speech intention where subsequent stimuli are read covertly. First, we expect that AWS and FC will show a stronger decrease in alpha and beta power during overt intention compared with covert intention as a marker of setting up the speech production network (Gehrig et al., 2012). Our second hypothesis relates to group differences in alpha and beta power. Currently, a stronger decrease in alpha and beta power (Mersov et al., 2016; Mock et al., 2016) or a reduced decrease in alpha and beta power (Jenson et al., 2018; Jenson et al., 2019) have been reported for AWS. Results either imply 
2. Increased right frontal beta power during speech intention in severe stuttering

a more strongly inhibited motor system or weakened sensorimotor integration. We expect that both functional states could already influence the setting up of the speech production network. Therefore, we hypothesize that a between-group difference should already emerge during overt speech intention, if it relates to an anatomical core instability of the speech production network and not to linguistic processing. On the other hand, if higher cognitive top-down processes, such as retrieving correct motor plans, are involved in this trait marker, we do not expect to see a group difference in alpha and beta power decrease during speech intention. We investigated hypothesis two by comparing the interaction between Group and Condition (hypothesis 2a) and the main effect of Group on overt speech intention prior to fluent speech (hypothesis $2 b$ ). Third, if the emergence of stuttering events relates to additional, e.g. linguistic, factors that destabilize the speech production network, we hypothesize that the oscillatory power should be the same for overt speech intention in both fluency states (stuttered vs. fluent speech). Contrarily, if stuttering events relate to internal processes of the speech production network, e.g. an increased inhibitory baseline level (e.g. Mersov et al., 2016; Wu et al., 1997), we expect that beta power during speech intention will differ between both fluency states. Again, we investigated hypothesis three by testing the interaction between Fluency State and Condition (hypothesis 3a) and the main effect of Fluency State on oscillatory power during overt speech intention (hypothesis 3b). Fourth, as we hypothesized that oscillatory power during speech intention and preparation should either show trait or state markers in AWS, we expected that stuttering severity would be linked with the decrease in oscillatory power during speech intention. Our EEG study is part of a larger experiment, which also investigated neural oscillations during self-initiated speech preparation (Korzeczek et al., in preparation) and speech performance on pseudowords (Chapter 3). 
2. Increased right frontal beta power during speech intention in severe stuttering

\section{Methods}

Participants

The ethical review board of the University Medical Center Göttingen, Georg August University Göttingen, Germany, approved the study, and all participants provided written informed consent before participation, according to the Declaration of Helsinki. Twenty adults with developmental stuttering (AWS) and 20 fluent controls (FC) were assessed. Exclusion criteria included any speech or language disorder other than developmental stuttering, any neurological impairment, drug abuse, medications that act on the CNS, nonnative speakers of German, or knowledge of Korean as a foreign language (Korean signs were included in our study design as described in the next section). We excluded the data of two participants. In one participant the neuroimaging MRI data necessary for the planned source analysis was not acquired. A second participant, who, after finishing the experiment, reported moderate depression that was being treated with antidepressant medication. The analysis, thus, comprised 19 participants in the stuttering group (three females, mean age $29 \pm 11.59$ ), and 19 fluent controls (three females, mean age $26.3 \pm 8.3$ ). The groups were comparable with regard to age, sex, education and handedness (Oldfield, 1971) (see Table 2). Stuttering started during childhood ( $<9$ years of age) as reported by all stuttering participants. Speech fluency was assessed prior to the experiment using the Stuttering severity index (SSI-4; Riley, 2009). Fluency assessments were performed by the first author and consisted of 300 syllables of spontaneous speech sample and overt reading text respectively. The group with participants with developmental stuttering included four participants with very mild stuttering, four with mild stuttering, six with moderate stuttering, one participant with severe stuttering and four participants with very severe stuttering. Interrater reliability was established by reanalyzing nine randomly chosen participants from each group by a second speech and language pathologist (N.E.N). Intraclass correlations of the SSI-4 scores yielded a good to excellent inter-rater reliability (total: ICC $=.95,95 \% \mathrm{CI}[.87$ $.98]$; reading: ICC $=.95,95 \% \mathrm{Cl}$ [.75 .99]; reading: ICC = .92, 95\% CI [.75 .98]; duration: 
2. Increased right frontal beta power during speech intention in severe stuttering

$\mathrm{ICC}=.83,95 \% \mathrm{CI}[.59 .93]$; concomitants: $\mathrm{ICC}=.78,95 \% \mathrm{CI}[.48 .91])$. No stuttering was observed in the control group. To assess the psycho-social impact of stuttering we used the German version of the Overall Assessment of the Speaker's Experience of Stuttering (OASES; Yaruss et al., 2016). In addition, group comparability for cognitive functions that can influence reading behavior was ascertained by administering seven additional subtests. Mental well-being was assessed by the mental well-being test (World Health Organization, 1998) and the Beck depression inventory (version 2, BDI-II, Beck et al., 1996). Working and short-term memory were tested with the digit span forwards and backwards subtest of the German version of the Wechsler adult intelligence scale - fourth edition (Petermann, 2012) and the Konsonanten Trigram Test (Consonant Trigram Test, Schellig \& Schächtele, 2002). To assess reading performance, the one-minute word and pseudoword reading test of the Salzburger Lese- und Rechtschreibtest-II (SLRT-II, Moll \& Landerl, 2010) was used. In two assessments, the digit span and the SLRT-II, task complexity was increased by using items with increasing sequence length; we therefore included stuttering severity as confounding covariate. The groups were comparable in all assessed categories (Table 2). Detailed information of assessments is provided in the supplement (appendix 1, Table 1 and 2). 
2. Increased right frontal beta power during speech intention in severe stuttering

Table 2. Demographic information of participants

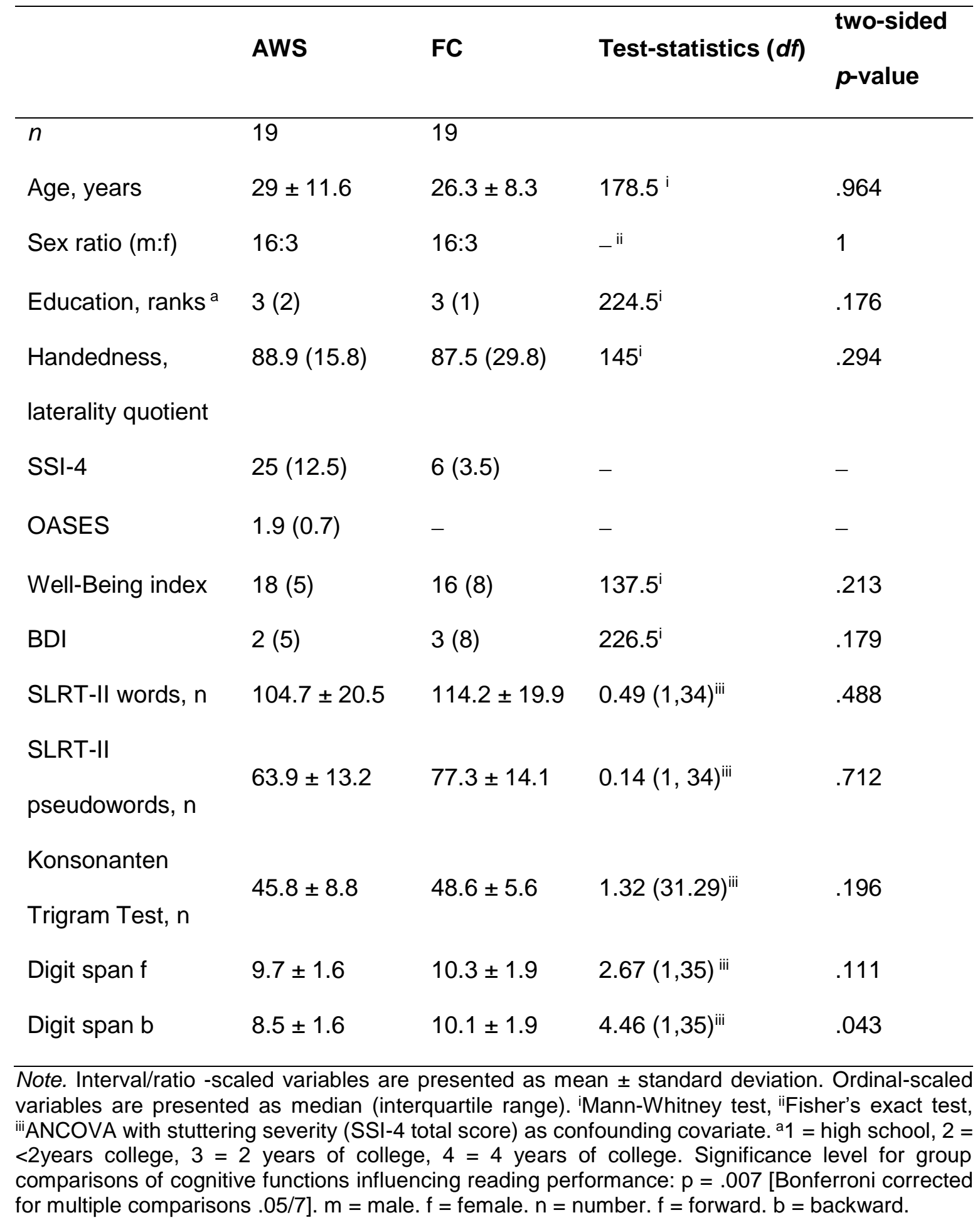


2. Increased right frontal beta power during speech intention in severe stuttering

\section{Stimuli and design}

We implemented a 2x2 design with the factors group (AWS vs. FC) and speech condition (overt vs. covert), using a cue-target reading paradigm to separate the intention to speak from linguistic processes such as speech motor planning and articulation (Gehrig et al., 2012) (Figure 2). The cue stimulus prepared the participants for either overt or covert speech production, whereas the target stimulus represented a pseudoword. The design was extended by a third non-speech condition, only during the speech preparation phase. Here, as a control task for covert speaking, $50 \%$ of the covert pseudowords were replaced by random Korean graphemes leading to visual processing of the stimuli without any preparation of speech production. The whole experiment consisted of eight blocks, each 12.5 minutes long and containing 75 trials. Thus, the participants conducted 300 covert and 300 overt trials. Covert and overt trials were equally distributed over the blocks. Each trial encompassed a baseline of two seconds duration, a two-second interval of speech intention, an interval for speech preparation and production, and a varying inter-trial interval (0.5 - to $1.5 \mathrm{~s}$, mean $0.8 \mathrm{~s})$ (Figure 2). Visual stimuli indicated task rules and marked conditions. A black circle represented the baseline and inter-trial interval, whereas the cue stimulus, a black or white filled cross, indicated overt or covert speech intention. Determined by the color of the cue stimulus, the participants either overtly or covertly read a subsequently presented pseudoword (target stimulus). We used 300 different pseudowords, which consisted of German syllables. Pseudowords were controlled for phonetic complexity, syllable frequency, and syllable length (see Chapter 3 for further details on pseudowords). 
Figure 2. Cue-target paradigm showing a trial of the overt condition.

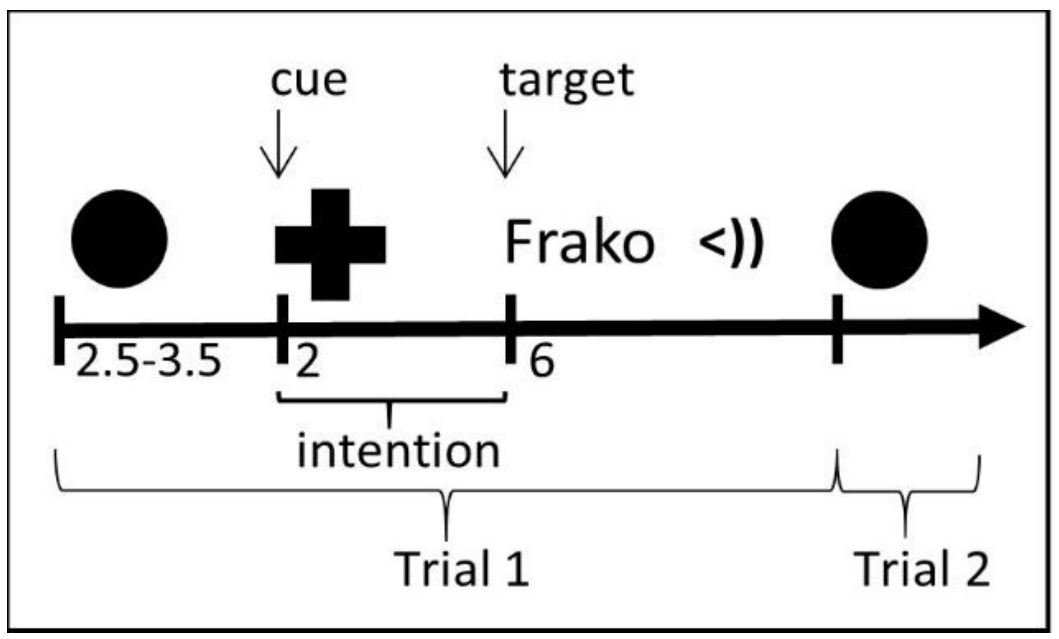

Note. The cue target marked the beginning of the speech intention condition. A pseudoword as target stimulus lead to self-initiated speech production: <)) ). The arrow indicates passage of time in seconds. Black circles represent baseline and inter-stimulus intervals.

\section{Procedure}

The participants were tested individually in a quiet room. Prior to the experiment assessments of speech fluency, short-term and working-memory were administered. Then participants were prepared for the experiment (taking head measurements, attaching the EEG and EMG electrodes, affixing the microphone, checking electrode impedance). During the experiment the participants sat in a comfortable chair at a distance of $90 \mathrm{~cm}$ to the computer screen. The experimental instructions and stimuli were presented visually using the experimental software PsychoPy, version 1.85.2 (Peirce, 2009; Peirce \& MacAskill, 2018). All stimuli were presented on a grey background at the center of the screen. To reduce eye movement artefacts, the participants were told to fixate the presented stimuli and to try to delay blinking until after having read the pseudowords. Written task instructions were presented prior to every second block. Task instructions informed the participants that a black cross meant that they were to read the following visually presented pseudoword out loud, whereas when a white cross appeared they should prepare for covert reading. To promote self-initiated speech, the participants were encouraged to wait for an internal urge to speak before overtly reading the presented pseudoword (McArdle et al., 2009). Because 
2. Increased right frontal beta power during speech intention in severe stuttering

speech stimuli consisted of unknown prosodic pseudowords, the participants should pretend that they were reading a German word. Lastly, the participants were instructed not to suppress stuttering events or to correct themselves. Before starting the experiment they went through ten practice trials that were not included in the analyses. After each practice trial, they were informed if they had made mistakes, i.e. incorrect task execution: silent speaking or body movements disturbing the EEG signal. The experimental blocks started when the participants signaled their readiness. During the experiment, in the middle of each of the eight blocks, the investigator gave a predefined verbal comment, e.g. "Please speak slightly louder", to maintain the participants' attention. The comments were the same for all participants, and the order of the comments was the same between groups. In addition, seven planned pauses of 2 to 8 min were inserted between blocks. The pauses allowed the participants to adjust their position, to drink, to remoist the electrodes (Coderre et al., 2017), and to assess further pretests, i.e. demographic information, reading performance and personal well-being. The sequences of pause durations and test assessments were the same for each participant. Immediately after the experiment, electrode positions and fiducials of the left and right periauricular points and the nasion were scanned. On a separate day, participants underwent a structural MRI scan but the data of these scans are not reported here.

\section{Acquisition and analysis of speech production}

The participants' speech production was recorded with a camera (Canon HF 100). During the experiment the video camera was positioned on the right side of the participant, so that the face and torso would be recorded. The distance of the camera from the participant was approximately 80 centimeters. In addition, the participants' speech was recorded with a wireless microphone (PT 40 Flexx pro, multi frequency) attached to participants' collar. Two speech and language pathologists each rated half of the video recordings for stuttering events and phonetic errors using ELAN, version 5.8 (Max Planck Institute for Psycholinguistics, Nijmegen, 2018). A verbal response was marked as stuttered or fluent 
2. Increased right frontal beta power during speech intention in severe stuttering

irrespective of how many stuttering events occurred during the response (Sasisekaran \& Weathers, 2019). Stuttering symptoms were defined as suggested by the SSI-4 (Riley, 2009). Due to technical issues, no video recording existed for one participant with very severe stuttering. This participant was analyzed by audio recordings, which can be taken into consideration if the stuttering symptoms are very distinct (Natke et al., 2004). For interrater reliability four randomly chosen participants (two of each group) were analyzed by both speech and language pathologists. Inter-rater reliability as calculated with Cronbach's alpha range between 0.65 and 0.68 . The first author checked all transcriptions for consistency.

\section{EEG data acquisition}

EEG data was obtained using a 256-sensor DC-EEG system (HydroCel Sensor Nets, Netstation Acquisition, Version 10.10.42, Electrical Geodesics, Inc. Eugene, OR), which has an integrated Physio 16 system for recording electromyography (EMG). Two electrodes, which were placed on the vermillion border at the upper and lower lip (McArdle et al., 2009; Salmelin et al., 2000), registered the activity of the onset of lip movements. Following the user manual and recommendations from Electrical Geodesics, fitting and preparing the nets included electrolyte preparation, head measurements and net placement on each subjects' scalp. The impedance of all electrodes was kept below $50 \mathrm{k} \Omega$ and checked prior to and during pauses in the experiment (Coderre et al., 2017; Ferree et al., 2001). The sampling rate was set to 1 kilohertz. To keep EEG data files at a transferable size, EEG data acquisition was stopped after every second block and there were, consequently, four EEG data files of each participant. Immediately after the experiment, electrode positions and fiducials of the left and right periauricular points and the nasion were recorded using Geodesics GeoScan. Pictures were taken of the fiducial points to enable a co-registration of EEG cap and the structural MRI of participants. For later head-modeling,

a structural T1- weighted MRI scan (magnetization prepared rapid gradient echo (MPRAGE) sequence: 45 sagittal slices, flip angle $=10^{\circ}, \mathrm{TR}=900$, voxel sized $3 \mathrm{~mm} \times 3$ 
2. Increased right frontal beta power during speech intention in severe stuttering

$\mathrm{mm} \times 3 \mathrm{~mm}, 210 \mathrm{~cm}$ FoV, 3 Tesla Siemens Magnetom Trio) was obtained of each participant after the experiment. However, source reconstruction of the EEG data will not be reported here.

\section{EEG data preprocessing}

To remove power line and direct current components a $50 \mathrm{~Hz}$ notch and a 0.01 to $160 \mathrm{~Hz}$ bandpass finite impulse response (FIR) filter were applied on continuous EEG data using Net Station version 4.3.1 (Electrical Geodesics, Inc.). Subsequent processing at the individual level were conducted with Fieldtrip toolbox (version 2017-11-08; Oostenveld et al., 2011) and custom scripts written in MATLAB (version R2018b, MathWorks, Inc.). We segmented EEG data into $10.5 \mathrm{sec}$ epochs (or trials). Each epoch was time-locked to the presentation of the task stimulus of speech intention (pre stimulus $-2000 \mathrm{~ms}$ and post stimulus $+8500 \mathrm{~ms}$ ). The ends of each trial were zero padded to increase the frequency resolution of Fourier transformations (Cohen, 2014). Next, trial information, including the chronological order of the trials and blocks, and condition, and the speech onset times of both lips in ms were added to the EEG data files. To identify speech onsets, we used EMG data. A notch filter (47 to $51 \mathrm{~Hz}$ and its harmonics) and a 2nd order Butterworth bandpass filter (110 to $140 \mathrm{~Hz}$ ) was applied on the demeaned EMG signal. A custom written automated script identified and marked speech onsets defined as one standard deviation of the squared EMG signal during a moving time window of 500ms (McArdle et al., 2009; Salmelin et al., 2000). Trials following a planned remark or including incorrect behavioral answers, such as overt instead of covert reading and vice versa, phonetic errors, or EMG signal onset below $300 \mathrm{~ms}$ after pseudo word presentation, were discarded. Because of muscle artefacts and bad skin contact, the face and neck electrodes (Figure 3) were excluded from further analyses, leaving 194 sensors per participant. After appending the four EEG recordings of each participant, the EEG data were downsampled to $500 \mathrm{~Hz}$. The trials were then redefined by cutting trial endings based on the identified fastest speech onset taking either the upper or lower lip signal. To detect and remove within-trial transients 
2. Increased right frontal beta power during speech intention in severe stuttering

(muscle and jump artifacts), a semi-automatic procedure with an adaptive threshold based on the z-score [cutoff z-value: 30 ] of the $110-140 \mathrm{HZ}$ bandpass filtered data was used. After muscle artefact detection, EEG data was filtered with a 2nd order $70 \mathrm{~Hz}$ Butterworth lowpass-filter. Using ft_rejectvisual, we removed noisy sensors before conducting an independent component analysis to delete artefacts due to eye movements, heartbeat and the temporalis muscle (Jung et al., 2000; Yilmaz et al., 2014). As a final checkup, the EEG data was again visually inspected with $\mathrm{ft}$ _rejectvisual. After, data cleaning, deleted sensors were replaced by spline-interpolation of neighboring sensors (Perrin et al., 1989). Then, the EEG data were rereferenced using common average. The groups did not differ in the number of incorrect responses, movement artefacts or bad sensors as indicated by a MANOVA using Pillai's trace, $V=0.81, F(3,34)=0.99, p=.408$ (for univariate ANOVAs of the individual dependent variables see Table 3). Data cleaning left 4,991 covert trials (mean $=262.7$, range $=217-286)$ and 3,843 fluent overt trials $($ mean $=202.3$, range $=136-240)$ in FC for statistical analyses. For AWS 4,909 covert trials ( mean $=258.4$, range $=207-280$ ), 2,912 fluent overt trials (mean $=153.3$, range $=13-223$ ) and 904 overt stuttered trials (mean $=47.6$, range $=3-131$ ) were kept .

Figure 3. Deleted face and neck electrodes of the EEG system.

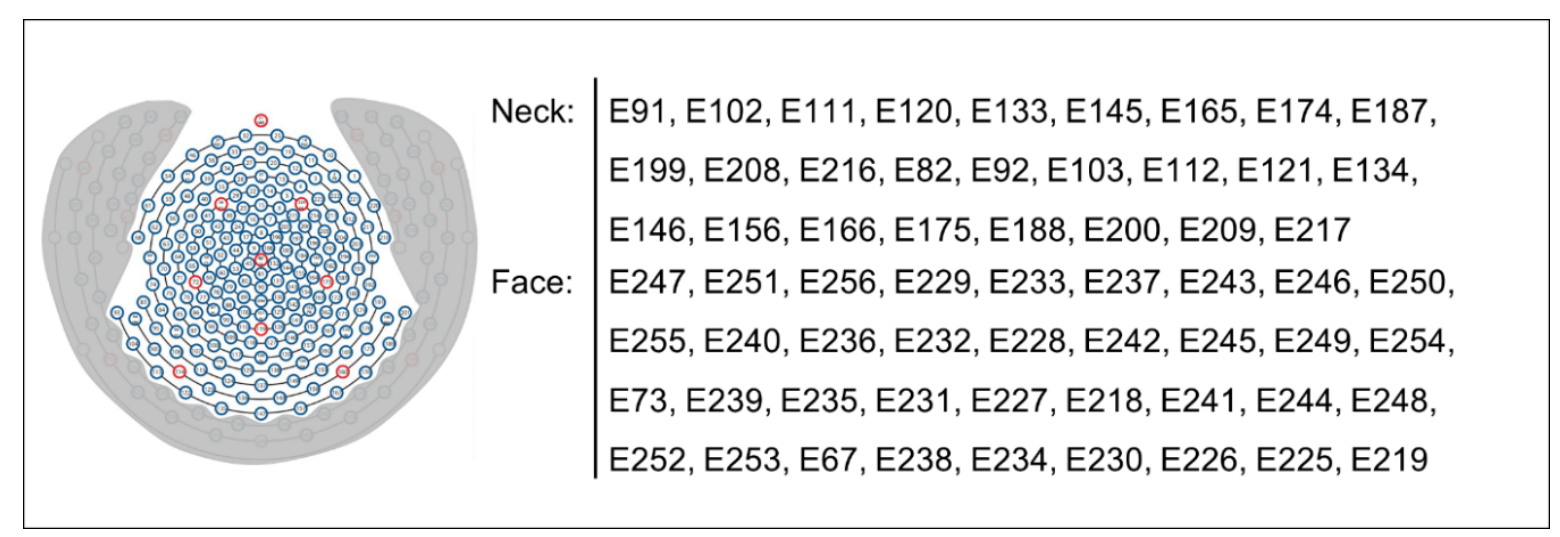

Table 3. No group differences on discarded EEG data

\begin{tabular}{llll}
\hline Artefacts & AWS - Mean (SD) & FC - Mean (SD) & Univariate ANOVAs
\end{tabular}


2. Increased right frontal beta power during speech intention in severe stuttering

\begin{tabular}{llll}
\hline Incorrect responses & $71.5(34.2)$ & $65.8(28.8)$ & $F(1,36)=0.31, p=.583$ \\
Movement artefacts & $39.4(21.9)$ & $40.8(18.7)$ & $F(1,36)=0.05, p=.825$ \\
Noisy sensors & $8.3(3.5)$ & $7.1(2.4)$ & $F(1,36)=1.68, p=.203$ \\
\hline
\end{tabular}

Time frequency analysis of power

We applied time-frequency analysis on sensor-level of stimulus and response locked EEG data over all conditions. For time-frequency representations of power, a frequencydependent sliding window (Hanning taper) based approach with zero padding of $16 \mathrm{~s}$ was used. The sliding window encompassed five cycles per frequency for the stimulus locked data relative to stimulus onset and three cycles per frequency for the response locked data. Power estimates were obtained from 8 to $30 \mathrm{~Hz}$ with a frequency resolution of $1 \mathrm{~Hz}$ and in time steps of $50 \mathrm{~ms}$. The time window of the time-frequency analysis depended on the zero offset of the data. Power estimates were extracted from -2000 to 4000 ms for stimulus locked data, relative to stimulus onset of speech intention, and from -4000 to $0 \mathrm{~ms}$ for response locked data, relative to speech onset. Prior to time frequency analysis of response locked data, data from -300 to $0 \mathrm{~ms}$ was reflected to ensure that the last $100 \mathrm{~ms}$ before speech onset were not lost (Cohen, 2014). Stimulus locked and response locked data were baseline-corrected per trial with the same baseline interval of -1000 to $0 \mathrm{~ms}$ before the stimulus onset for the speech intention condition using relative change to baseline (EEG mean(baseline)./ mean(baseline)).

\section{Statistical analysis}

We examined power changes during speech intention during the first $1000 \mathrm{~ms}$ after cue onset (Gehrig et al., 2012). We investigated i) effects of Condition (e.g. overt vs. covert) within each Group, ii) between Group effects (AWS vs FC) within each Condition (e.g. overtfluent $_{\text {or covert), iii) effects of Fluency State (overtfluent }}$ vs. overtstuttered) within AWS, and iv) the influence of stuttering severity on overtfluent and overt $_{\text {stutterd. }}$ In addition, prior to 
2. Increased right frontal beta power during speech intention in severe stuttering

calculating main effects of Group and Fluency State, we also investigated $\Delta$-between Group and $\Delta$-between-Fluency State effects by comparing the difference of the overt condition of interest minus the covert condition, i.e. the interactions between Condition and Group or Fluency State. Within-Group comparisons were conducted with dependent t-tests, whereas between-Group comparisons were calculated with independent t-tests. To investigate the influence of stuttering severity (rank of stuttering severity, SSI-4) on oscillatory power during overt speech conditions (intention and preparation), we calculated regressions using the function ft_statfun_indepsamplesregrT. Statistics were run separately for individual frequency bands (Brinkman et al., 2014). Frequency bands, alpha (8-13 Hz), low beta (15$25 \mathrm{~Hz}$ ) and high beta $(25-30 \mathrm{~Hz})$, were defined based on previous studies (Gehrig et al., 2012; Liljeström et al., 2015; Mersov et al., 2016). All statistical analyses were conducted with cluster-based permutation tests using Monte Carlo cluster (two-tailed testing). To correct for multiple testing of the three frequency bands, we considered $p$-values below 0.017 as significant, (0.05/3). To improve the signal-to-noise ratio for statistical analyses, participants with fewer than 20 trials in the respective condition were excluded. This restriction accounted for one AWS in the fluent and for five AWS in the stuttered condition, leaving 19 AWS for between-group analyses and 13 AWS for within-group analyses. Because one AWS was fluent in fewer than 20 trials, this participant was excluded from statistical analyses examining the oscillatory power of fluent overt speech conditions. The same threshold of 20 trials was applied on further five AWS, who were excluded from statistical analysis examining oscillatory power of stuttered overt speech conditions.

\section{Results}

Results pertaining to hypothesis 1: Between condition contrast of overt vs. covert speech intention within both groups

Both groups showed statistically significant reduced alpha and low beta power during overt compared with covert speech intention (all $p<.001$ ). High beta power did not differ between 
2. Increased right frontal beta power during speech intention in severe stuttering

conditions in either group. Considering alpha and low beta power, hypothesis one was fulfilled. Figure 4 shows significant time-frequency clusters and the scalp distribution of their difference between overt and covert speech intention. During overt versus covert speech intention, FC showed reduced alpha power during the entire time window of interest ( $1 \mathrm{~s})$ as well as across the complete frequency band. Alpha power spread over bilateral posterior, central and anterior sensors. AWS showed reduced alpha power mainly within upper frequencies of the alpha band $(10-13 \mathrm{~Hz})$, peaking between $300 \mathrm{~ms}$ and $700 \mathrm{~ms}$ after stimulus presentation over bilateral posterior and mid central sensors. In both groups, reduced alpha power was detected in sensors over right temporal regions, whereas only FC showed a reduction in frontal bilateral sensors (Figure 4, upper row). Reduced low beta power during overt versus covered speech intention occurred between $150 \mathrm{~ms}$ to $600 \mathrm{~ms}$ after cue onset in both groups (Figure 4, lower row). The decrease was more pronounced in lower frequencies of the low beta band, i.e. 15 to $20 \mathrm{~Hz}$. Scalp maps show that the low beta power difference emerged first over anterior central sensors, spreading then to sensors over left parietal and temporal regions. 
2. Increased right frontal beta power during speech intention in severe stuttering

Figure 4. Reduced alpha and beta power during overt speech intention.

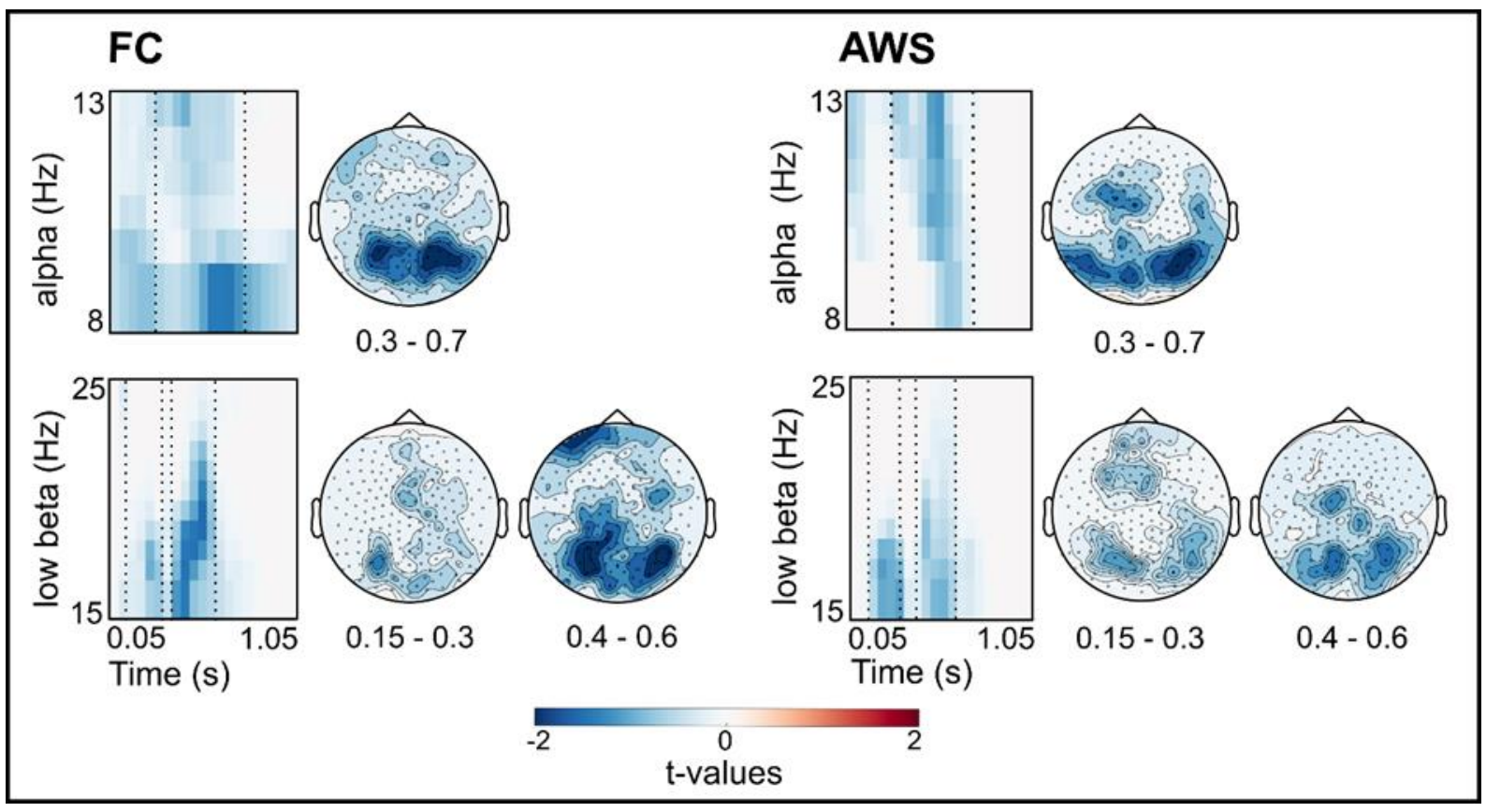

Note. Time frequency plots (cluster corrected, $p<.017$ ) show significant alpha and low beta power differences between the overt and the covert speech intention over averaged sensors for FC and AWS separately. Dashed lines mark the time window of scalp maps. Black dots in scalp maps represent EEG sensors. FC, n: 19, AWS, n: 18.

The reported reductions in alpha and low beta power only exhibited a decreased power relative to baseline in the posterior EEG sensors (Figure 5). In the anterior EEG, the reported power reductions, represent a less strong increase in relative power during overt than during covert speech intention (Figure 5). 
2. Increased right frontal beta power during speech intention in severe stuttering

Figure 5. Descriptive alpha and low beta power during speech intention.

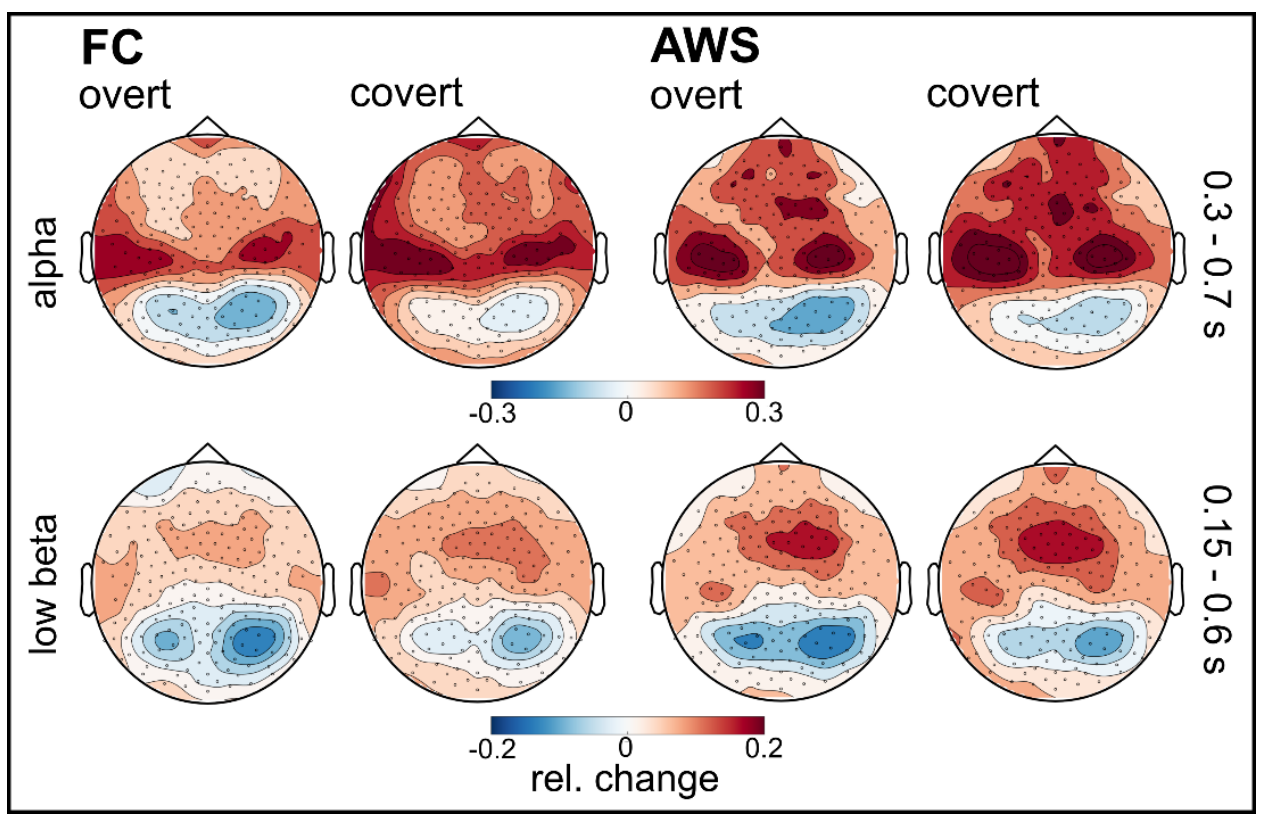

Note. Scalp maps show alpha and low beta power as relative change to baseline for both groups separately. Outer right column: time windows for scalp maps.

Black dots represent EEG sensors. FC, n: 19, AWS, n: 18.

Results pertaining to hypothesis 2: Between group contrast of overt intention prior to fluent speech

Neither the interaction between group and condition nor the main effect of group were statistically significant (all $p>$.017). Hence, hypothesis two was rejected.

Results pertaining to hypothesis 3: Between fluency state contrast during overt speech intention in AWS

Neither the interaction between fluency state and condition nor the main effect of fluency state were statistically significant (all $p>$.017). Thus, alpha and beta power of AWS during overt speech intention did not differ between subsequent fluency states (fluent vs. stuttered). Hence, hypothesis three was fulfilled; we rejected the alternative hypothesis.

Results pertaining to hypothesis 4: Stuttering severity is linked with beta power decrease during speech intention

Stuttering severity showed a significant influence on low beta power of overt speech intention prior to fluent speech, $p=.007$ and a trending effect on low beta power of overt 
2. Increased right frontal beta power during speech intention in severe stuttering

speech intention prior to stuttered speech $(p=.034)$ (Figure 6). In both fluency states, AWS with more severe stuttering had a stronger low beta power increase over central regions. In addition, AWS with more severe stuttering showed higher low beta power over left parietal and right temporal regions prior to fluent speech (Figure 6). Hence, hypothesis four was rejected.

Figure 6. Influence of stuttering severity on low beta power during overt speech intention

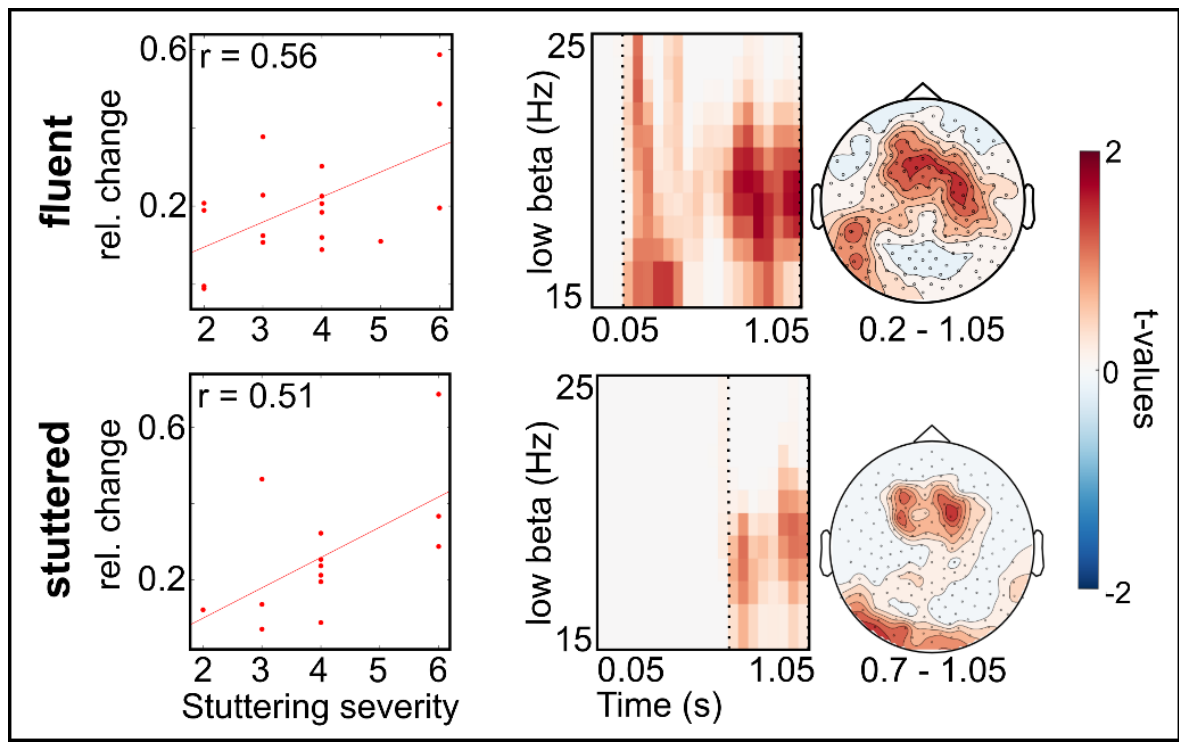

Note. The upper row shows low beta power (cluster corrected with $p<.017$ ) prior to fluent speech (AWS, n: 18). The lower row shows low beta power (cluster corrected with $p<.05$ ) prior to stuttered speech (AWS, n: 13). Left column: scatterplots show the averaged low beta power relative to baseline of each AWS according to stuttering severity (rank of stuttering severity of SSI - IV) and the respective correlation coefficient. Stuttering severity ranks: $2=$ very mild, $3=$ mild, $4=$ moderate, $5=$ severe, $6=$ very severe. Central column: time frequency plots over averaged sensors. Dashed lines in time frequency plots mark the chosen time window for scalp maps. Right column: scalp maps show low beta power averaged over relevant time window. Black dots represent EEG sensors.

\section{Discussion}

This sensor-level EEG study investigated alpha and beta power in AWS during the setting up of the speech production network, i.e. speech intention. A cue-target reading paradigm separated activation of the speech production network from linguistic processing. 
2. Increased right frontal beta power during speech intention in severe stuttering

Compared with the intention of covert reading, alpha $(8-13 \mathrm{~Hz})$ and low beta $(15-25 \mathrm{~Hz})$ power was reduced in both groups during overt speech intention. However, a decrease in alpha and beta power relative to baseline appeared only in posterior sensors covering parietal regions. Contrary to our expectations, main effects of group, fluency state or interactions between group and condition or between fluency state and condition were not statistically significant. Nevertheless, in the AWS group persons with more severe stuttering, as indicated by the SSI-4 total score, showed significantly increased low beta power during speech intention prior to fluent overt speech. Increased low beta power was detected in sensors covering left parietal, central and right temporal regions. A trend indicated that persons with more severe stuttering also showed stronger low beta power over central regions during speech intention prior to stuttered speech.

\section{Activation of the speech production network during speech intention}

Prior to knowing what to say, thus prior to linguistic processing, the brain already prepares for overt speech production. The current study replicates previous findings (Gehrig et al., 2012) by showing that alpha and slow beta power are more strongly reduced in FC during the intention to read the subsequent pseudoword overtly compared with covertly. In general, a reduction in oscillatory power has been linked to disinhibition of the respective cortical region (Jenkinson \& Brown, 2011; Klimesch, 2012) or in case of the beta band to a "change in the cognitive or perceptual set" (Engel \& Fries, 2010). In the current study, AWS show a reduction in alpha and low beta power while intending to speak overtly as FC. Thus, also in AWS preparation of overt speech induces a stronger change in the cognitive and sensorimotor set compared with covert speech.

For both groups, the time-frequency plots of the results revealed two peaks of reduced low beta power with different topographic patterns between 150-300 ms and 400-600 ms after stimulus onset during overt compared with covert speech intention. During cue-target paradigms the observed beta band power modulation can relate to different processes encompassing motoric and cognitive functions (Kilavik et al., 2013). During 150 to $300 \mathrm{~ms}$ 
2. Increased right frontal beta power during speech intention in severe stuttering

after cue onset, low beta power appeared primarily over mid anterior, right central and bilateral posterior sensors covering thus the medial frontal cortex. We suggest that the activation differences over mid anterior sensors could stem from the medial frontal cortex and the anterior cingulate gyrus, which are important for cognitive top-down regulation to implement the task-dependent cognitive state (Dosenbach et al., 2006). The supplementary motor area as part of the speech production network, is linked to speech initiation (Guenther \& Vladusich, 2012; Kotz \& Schwartze, 2010). Further, covert intention demanded of participants to suppress overt articulation for the following pseudoword. Beta power increases in the right inferior frontal gyrus and right supplementary motor area was strongly related to movement inhibition (Picazio et al., 2014; Swann et al., 2009). Thus, the observed activity cluster could indicate a stronger preparation of movement inhibition during covert compared with overt intention.

During the second time window, 400 to 600 ms after cue onset, reductions in low beta power appeared over left and mid central and parieto-occipital sensors, including the CZ and C3 sensors. Thus, the EEG covered motor-related regions such as the precentral gyrus. This activation pattern could indicate the preparation for a movement change (Brittain \& Brown, 2014; Engel \& Fries, 2010) and is in line with previous studies reporting a decrease in beta power in motor regions, even though no direct cue for motor planning was given (Gehrig et al., 2012; Zaepffel et al., 2013). We, therefore, suggest that the low beta power reduction in this study reflects cognitive and motor processes required for task implementation and early movement preparatory processes. The restricted time window from 150 to $600 \mathrm{~ms}$ is in contrast to the results of Gehrig et al. (2012), who reported reduced alpha and beta power from 350 to 1000 ms post stimulus. In general, movement studies show that early beta power decrease after cue onset can either change into a subsequent increase (Alegre et al., 2006; Fischer et al., 2010; Klostermann et al., 2007) or persist until the presentation of the target stimulus (Mersov et al., 2016; Pastötter et al., 2012; Saleh et al., 2010; Tzagarakis et al., 2010; Zaepffel et al., 2013). Our time-limited beta decrease could be explained by 
2. Increased right frontal beta power during speech intention in severe stuttering

the present experiment design: for both speech conditions the target cue, i.e. pseudoword, appeared after two seconds leading to a timed anticipation of the target stimulus. The anticipatory increase in beta power prior to a timed cue (Fujioka et al., 2012; Saleh et al., 2010), could have masked differences between both conditions, i.e. the beta power decrease prior to the target cue of overt speech production.

In addition to reduced low beta power, alpha power was also reduced during overt versus covert speech intention. Reduced alpha power could account for an anticipatory activation of the speech production network (Gehrig et al., 2012). The source of reduced alpha power during speech preparation has been assigned to left temporal auditory regions (Gehrig et al., 2012; Jenson et al., 2015; Mersov et al., 2016). Regarding changes in alpha power as a reflection of attentional focus (Klimesch, 2012), a reduction in alpha power over auditory regions indicates their disinhibition and increased attention on expected or perceived auditory input (Drijvers et al., 2018; Jensen \& Mazaheri, 2010; Kerlin et al., 2010). Our results show a wide topographical distribution of alpha power reduction: from central and left hemisphere anterior sensors to reduction peaks in left and especially right posterior EEG. The topographic distribution is similar to the one reported by Gehrig et al. (2012). However, as we do not report a source analyses, the observed reduction of alpha power in this study could also emerge from occipital and parietal regions rather than from auditory regions. During reading, alpha power decreases over posterior sensors (Pfurtscheller et al., 1994). Reduced pre-stimulus alpha power in occipital and parietal regions has been linked to increased anticipatory attention to an upcoming visual event and successful visual perception (Engel \& Fries, 2010; Ergenoglu et al., 2004; Hanslmayr et al., 2007; Romei et al., 2010). Further, several fMRI studies observed increased left lateralized activation in occipital areas during pseudoword reading (e.g. Bouhali et al., 2019; Price, 2012; Taylor et al., 2013; Woollams et al., 2011). As increased attentional focus led to lateralized alpha on the contralateral side (Klimesch, 2012), the descriptive stronger reduction in alpha power in right posterior sensors (Figure 5) could indicate anticipation of visual pseudoword 
2. Increased right frontal beta power during speech intention in severe stuttering

processing. We suggest that visual anticipation was less strong during covert intention, as subsequent target stimuli could be either pseudowords or non-readable Korean signs. Thus, the observed decrease in alpha power in our study could also implicate the anticipation of reading unknown pseudowords aloud.

\section{Stuttering severity is linked with stronger beta power during speech intention}

We found a significant association between stuttering severity and low beta power increase during speech intention prior to fluent speech. Thus, the more severely a person stuttered the stronger the increase in low beta power in the mid anterior and right central EEG. Our finding is contrary to that of Mock et al. (2016), who reported a stronger decrease in beta power with more severe stuttering during speech planning. The differing results might be explained by differences in the study design, speech intention prior to any linguistic processing vs. speech planning.

Interestingly, the time-frequency representation of our result revealed that beta power increase was less strong between 500 and 650 ms after cue presentation. This time window coincided with the reduced beta power in the overt vs covert comparison, which we interpreted as a preparation for movement change implemented via the direct pathway of the basal ganglia (Jenkinson \& Brown, 2011). Behavioral studies show that low beta power increases prior to anticipated timed cues (e.g. Saleh et al., 2010), during working memory tasks (e.g. Schmidt et al., 2019) and during movement inhibition (e.g. Jenkinson \& Brown, 2011).

Two arguments speak against increased anticipation of the target stimulus in AWS with more severe stuttering. First, beta power increase to a timed cue is mainly observed in close proximity to the anticipated cue and not across the entire time period as observed here (Fujioka et al., 2012; Saleh et al., 2010). Second, a recent study reported a beta power decrease prior to timed auditory cues in children with developmental stuttering (Etchell et al., 2016). Further, we would like to clarify that the observed increase in beta power with increasing stuttering severity prior to overt fluent speech does not account for the concept 
2. Increased right frontal beta power during speech intention in severe stuttering

of stuttering anticipation, which refers to the ability of persons with developmental stuttering to anticipate a stuttering event (Bloodstein \& Ratner, 2008; Garcia-Barrera \& Davidow, 2015).

Stronger motor inhibition in AWS with more severe stuttering could account for the observed beta power increase (Mersov et al., 2016; Mersov et al., 2017). Increased beta power has been associated with movement termination or successful withholding, with movement slowing and with tonic muscle contractions (Gilbertson et al., 2005; Pfurtscheller et al., 2005; Piai et al., 2015; Picazio et al., 2014). Topographically, we observed a stronger beta power increase in mid and right anterior and bilateral central sensors. Furthermore, especially the right hemisphere, the inferior frontal gyrus and pre supplementary motor area have been repeatedly associated with successful movement inhibition (Jahfari et al., 2011; Swann et al., 2012). In addition, it has been shown that the right inferior frontal gyrus modulates motor cortex excitability via beta oscillations (Picazio et al., 2014). Similar to our results, Mersov et al. (2016) observed a greater beta power increase in the left mouth motor cortex in AWS compared with FC before the next cue stimulus (a pseudoword) and after fluent speech production. However, in this study stuttering severity was not associated with beta increase. The differing results of the two studies might be explained by the different grades of stuttering severity in the AWS group: in our study stuttering severity ranged from very mild (four participants) to very severe (three participants), while in the study of Mersov et al. (2016) it ranged from mild to severe (three participants). Thus, the full range of stuttering severity in our study may have enabled a better association between stuttering severity and beta power. Increased levels of beta power and thus increased movement inhibition are also reported in Parkinson's disease, a movement disorder, and linked to decreased dopaminergic levels in the cortico-basal-ganglia loop (Jenkinson \& Brown, 2011). The correlation of low dopaminergic level in the basal ganglia with subcortical and cortical beta power increase, is not entirely consistent with our finding, as previous neuroimaging and pharmacological studies reported a hyperdopaminergic system in 
2. Increased right frontal beta power during speech intention in severe stuttering

stuttering (Maguire et al., 2004; Maguire et al., 2010; Watkins et al., 2008; Wu et al., 1997). Etchell et al. (2014) hypothesized that cortical hyperactive beta power in AWS could reflect a compensatory mechanism for dysfunctional beta activity at the striatal level. The fact that beta oscillations can also be generated at a cortical level (Schmidt et al., 2019) and that movement inhibition can be implemented via a corticocortical network (Picazio et al., 2014) could then account for our finding of stronger cortical beta power increase with increasing stuttering severity irrespective of basal ganglia functionality. Thus, we suggest that our results may indicate a less pronounced task set of overt speech production in AWS with more severe stuttering. This interpretation is supported by the fact that a similar trend emerged for the stuttered condition, although this was based on fewer trials and participants.

The observed right hemispheric increase in beta power could also be related to increased executional control (Keller \& Kell, 2016). In a cognitive domain, beta power increase has also been suggested to prevent interference from distraction or to maintain the current contents during working memory tasks in prefrontal areas (Schmidt et al., 2019). We suggest that the right lateralization of oscillatory power is more indicative of an inhibitory process, but only a source analysis of the data would reveal whether regions related to motor inhibition or cognitive control contribute to the observed pattern.

No significant differences, neither between groups nor between fluency states

We did not find any difference in alpha or beta power between groups or between fluency states. We discuss both null results separately.

By investigating oscillatory power modulations during speech intention in trait contrasts, we intended to resolve whether structural anomalies in AWS contribute to a basic functional deviation within the speech production network. Our results showed no group differences during speech intention prior to overt fluent speech. Based on our hypothesis we were able to accept the alternative hypothesis and concluded that the set-up of the speech production network, measured with oscillatory power, is similar in both groups and that trait differences 
2. Increased right frontal beta power during speech intention in severe stuttering

only emerge during speech preparation. This suggestion would further be in line with the result of similar oscillatory power levels in AWS compared with ANS in the resting state (Joos et al., 2014). However, this conclusion might be too hasty, as methodological differences regarding EEG analysis method, group size, and the range of stuttering severity could have contributed to our finding. For example, the current sensor analysis compared 18 AWS with very mild to very severe stuttering with 19 ANS. The only other study investigating alpha and beta power across the whole range of stuttering severity investigated 24 AWS with a more focused analysis method, i.e. comparison of sensorimotor components (Jenson et al., 2018).

Next, we hypothesized that beta power during speech intention should differ between both fluency states if stuttering events in AWS were related to an altered activation of the speech production network underlying endogenous sensorimotor rather than speech preparation processes. Again, at first glance, our results indicated that beta power relevant for setting up speech production did not contribute to later states of fluency. Two studies investigated fluency state related beta power modulation in AWS during speech preparation, one using a analysis approach similar to ours did report similar beta powers between fluency states (Mersov et al., 2017). Thus, irrespective of linguistic stimuli both studies investigating the relative change in beta power could not detect differences between fluency states. However, a study using a different analysis approach similar to machine learning reported differences in trait and state stuttering in eight AWS with an average of 40 stuttered trials per participant (Sengupta et al., 2017; Sengupta et al., 2019). We therefore suggest that more studies with either larger group sizes, consistent stuttering severity, or hypothesis driven ROI-analyses be conducted to elucidate the link between oscillatory power and stuttering.

\section{Low and high beta}

The present study differentiated low $(15$ to $25 \mathrm{~Hz}$ ) and high (25 to $30 \mathrm{~Hz}$ ) beta oscillations. This differentiation of the two ranges stemmed from the fact that an earlier study on 
2. Increased right frontal beta power during speech intention in severe stuttering

stuttering used 15 to $25 \mathrm{~Hz}$ as a beta range (Mersov et al., 2016), whereas another study investigating the entire frequency range of 14 to $30 \mathrm{~Hz}$ described a beta power decrease between 25 to $30 \mathrm{~Hz}$ in anterior sensors during overt speech intention (Gehrig et al., 2012). Further, the high beta frequency range induced coherence between sensorimotor regions of speech production during speech preparation (Liljeström et al., 2015). In addition, studies on reaction time exhibited stronger low beta power $(15$ to $25 \mathrm{~Hz}$ ) over the motor cortex prior to fast reactions (Pastötter et al., 2012). It is suggested, that low beta power $(<20 \mathrm{~Hz}$ ) may be related to movements, whereas high beta $(>20 \mathrm{~Hz})$ could reflect attention and anticipation (Schmidt et al., 2019). Interestingly, the present study revealed that only low beta power differed between overt and covert conditions and correlated with stuttering severity.

\section{Limitation of the current study}

The interpretation of the current results are limited to the topographic power distribution overt the scalp, as we did not report source localizations of the oscillatory activity. The slight variations in individual brain structures and the inter-subject variability of sensor placing made it difficult to infer the brain sources of oscillatory activity from sensor-level EEG data (Tzagarakis et al., 2010). Source and virtual sensor analyses in the frequency bands of interest are planned.

Second, we applied a trial by trial baseline normalization to account for the fact that alpha oscillations increase in their amplitude over time (Benwell et al., 2019). However, this kind of baseline normalization could have induced a rather decreased signal to noise ratio as rare signal changes were not averaged out.

Last, the participants were allowed to prepare their response without any time pressure after the target stimulus. Although the groups did not differ in their response reactions times for fluent trials, self-initiated reading might have reduced the urge for sensorimotor preparation prior to the target stimulus. 
2. Increased right frontal beta power during speech intention in severe stuttering

\section{Conclusion}

The present study revealed that both AWS and FC show a reduction in oscillatory power in relation to the intention of overt compared with covert reading. This indicates the set-up of the relevant brain network for overt reading. Further, AWS with more severe stuttering seem to show a stronger maintenance of the current cognitive and/or sensorimotor state, as stuttering severity was associated with increased beta power. Increase beta power levels may influence subsequent speech preparation and execution processes.

\section{Acknowledgements}

This study is part of the PhD dissertation of Alexandra Korzeczek at the Georg-August University Göttingen. We are grateful to Devran Yildirim for contributing to the evaluation of EEG data. 


\section{Effects of word length and syllable frequency in adults}

\section{with developmental stuttering}

Authors: Alexandra Korzeczek ${ }^{1}$, Jana Wiechmann², Katharina Hammers ${ }^{2}$, Nicole E. Neef $^{1}$, Arno Olthoff ${ }^{3}$, Martin Sommer ${ }^{1,4}$, and Joana Cholin ${ }^{2}$

\section{Affiliation:}

1Department of Clinical Neurophysiology, University Medical Centre Göttingen, Göttingen, Germany;

${ }^{2}$ Faculty of Linguistics and Literary Studies, Bielefeld University, Bielefeld, Germany; ${ }^{3}$ Department of Phoniatrics and Pedaudiology, University Medical Centre Göttingen, Göttingen, Germany

${ }^{4}$ Department of Neurology, University Medical Center Göttingen, Göttingen, Germany.

$$
\text { will be submitted shortly }
$$

\section{Authors contributions:}

A.K., M.S., and J.C. conceived and designed the experiment; A.K. administered the project, performed the experiment, analyzed and visualized the data; A.K., J.W. and K.H. evaluated the data; N.E.N. contributed to data analysis and interpretation of the results; A.O. contributed to the coordination of the study and gave recommendations on data evaluation; A.K. and J.C. interpreted that data, wrote and revised the manuscript, all authors reviewed the manuscript. 
3. Effects of word length and syllable frequency in adults with developmental stuttering

\begin{abstract}
Syllable frequency effects have been interpreted as evidence for stored syllable motor programs being retrieved during phonetic encoding to facilitate spoken production planning. However, these have never been tested on stuttering. The present study investigates effects of syllable frequency and word length on fluency and accuracy in 19 adults with developmental stuttering (AWS) and 19 fluent controls. Participants produced pseudowords of different length (two-, three- and four-syllabic words) with high- and low-frequency first syllables. We expected high-frequency syllables to be uttered more fluently and with greater accuracy compared to low-frequency syllables. A significant effect of word length but no effect of syllable frequency was obtained regarding speech fluency in AWS. However, response accuracy yielded a significant Group by Syllable Frequency interaction. AWS produced more errors on pseudowords containing high-frequency first syllables. In addition, we show that word length affects initial response accuracy. The results are discussed against the background of current production models and a new account for phonetic encoding processes in AWS is put forward.
\end{abstract}


3. Effects of word length and syllable frequency in adults with developmental stuttering

\section{Introduction}

Developmental stuttering is a speech fluency disorder of unresolved origin. Primary symptoms are involuntary speech blocks, sound and syllable repetitions and prolongations of speech sounds. Depending on severity, stuttering can markedly hamper fluent speech production. Adults with developmental stuttering (AWS) internally know what to say, i.e., they do not suffer from an inability to retrieve intended meanings or corresponding lexical items but have trouble to produce speech fluently (for an overview see Bloodstein \& Ratner, 2008). While there is, to date, no comprehensive cognitive account of the underlying causes of stuttering, several psycholinguistic factors have been associated with stuttering.

Brown (1938a, 1945) was among the first who found that stuttering is sensitive to several linguistic units, including the grammatical function of words: AWS are more likely to stutter on content words compared to function words (Au-Yeung et al., 1998; Hartsuiker et al., 2005; Howell et al., 1999; Max et al., 2019). It is not entirely clear how the lexical status correlates with other factors found to be critical in stuttering such as position within words and sentences, syntactic and phonetic complexity and word length. Linguistic elements in initial positions, i.e., first phonemes or syllables in words and or initial words in sentences seem to be more susceptible to stuttering than later elements (e.g. Au-Yeung et al., 1998; Hartsuiker et al., 2005; Howell et al., 1999). Syntactically more complex structures have been reported to inflict more stutter occurrences than less complex ones (Kadi-Hanifi \& Howell, 1992; Logan \& Conture, 1997; Melnick \& Conture, 2000; Ratner \& Sih, 1987; Yaruss, 1999). Likewise, Howell and colleagues (Al-Tamimi et al., 2013; Dworzynski \& Howell, 2004; Howell et al., 2006; Howell \& Au-Yeung, 2007; Sheehan, 1974) present cross-language evidence that the phonetic complexity of a word is linked to higher stuttering rates for adolescents and adults (see Coalson et al., 2012 for counter-evidence in children). Moreover, effects of word length constitute that longer words, either measured in number of phonemes or syllables, evoke higher stuttering rates compared to shorter words in AWS (Brown, 1938a, 1945; Logan \& Conture, 1995; Max et al., 2019). Another factor that has 
3. Effects of word length and syllable frequency in adults with developmental stuttering

been found to affect the occurrence of stuttering is word frequency, i.e. the occurrence of a specific word in a given corpus. AWS stutter more on low-frequency words compared to high-frequency words (e.g., (Anderson, 2007; Hubbard \& Prins, 1994; Newman \& Bernstein Ratner, 2007; Palen \& Peterson, 1982; Ronson, 1976). Brown (1938b, 1945) as well as Wingate $(1988,2002)$ pointed out that stuttering is even more likely when these factors coincide on one item. It should be noted though that these factors are not independent of one another: while longer and more complex words and structures tend to be less frequent, shorter and less complex entities are more frequent (Sigurd et al., 2004; Strauss et al., 2006). It needs to be carefully disentangled whether these factors are additive or whether they interact with one another (for different approaches see e.g. Nickels \& Howard, 2004; Ziegler, 2009). The next crucial step then is to pinpoint the language encoding level(s) on which these effects can be located. While the above described effects have largely been ascribed to lexical units and processes, within cognitive (Levelt et al., 1999), the emergence of stuttering has been associated with later, post-lexical encoding levels where linguistic planning interfaces with motor programming, sensorimotor integration and execution (Civier et al., 2010; Civier et al., 2013; Howell, 2004; Max, Guenther et al., 2004; Packman et al., 2007). Here, stuttering has repeatedly been explained by a temporal misalignment between subsequent processes such as a failed interplay at the phonological/phonetic (Postma \& Kolk, 1993), or the phonetic/articulatory interface (e.g. Civier et al., 2013; Packman et al., 2007), respectively. A unit that is known to play a functionally important role in fluent speakers on these levels is the syllable. However, even though there is a wealth of evidence (see following section) that syllables facilitate in smooth and rapid speech planning, syllabic effects have not been examined thoroughly in AWS. Let us first turn to the process of wordform encoding in fluent speakers before we will introduce the current study that set out to investigate syllable effects in AWS. 
3. Effects of word length and syllable frequency in adults with developmental stuttering

\section{The process of word-form encoding}

Word-form encoding starts by retrieving the word's single morphemes (e.g., rose (stem) + $\mathrm{s}$ (+plural) to form /roses/). The morphemes themselves consist of even smaller building blocks, i.e., phonemes that are spelled out and grouped together during the next encoding step: phonological encoding. Here, single phonemes are bundled together to form abstract phonological syllables applying universal and language-specific syllabification rules. Only in cases of irregular stress, a stored pattern is retrieved from memory. Syllabification procedures assign incoming phonemes to syllable-internal positions one-by-one, starting from the left edge and proceed stepwise to the right edge of a to-be-prosodified phonological word. The assignment of phonemes to a syllabic slot initiates phonetic encoding. During phonetic encoding, abstract phonological units are conversed into context-dependent phonetic units or motor programs. Within the framework of the mental syllabary (Levelt et al., 1999; Levelt \& Wheeldon, 1994) there are two possible pathways to go from phonological units to phonetic ones: a retrieval route and an assembly route. Via the retrieval route, precompiled phonetic syllables are accessed from a hypothesized mental syllabary. The syllables within the syllabary will not only receive activation from the addressing phonemes but also by activation-spreading inside the syllabary in which neighboring syllables (i.e., those units with shared phoneme positions) compete for selection. Selection takes place via a verification rule that checks the activated motor programs inside the syllabary against the addressing phonological syllable (Levelt et al., 1999). The success of this matchmaking crucially depends on a) identifying a syllable boundary, i.e., the right edge of the addressing syllable and b) the speed with which the corresponding syllable gets activated.

Whereas the retrieval route offers a short cut to stored precompiled syllable programs that facilitates fast and accurate phonetic encoding, speakers must have another mechanism to construct syllables segment by segment. Such an assembly route is likely to be more resource-costly, slower and more error-prone than the retrieval route, speakers can use this 
3. Effects of word length and syllable frequency in adults with developmental stuttering

route in case of new (e.g. in foreign language learning) or low-frequency syllables that are not part of the stored inventory. This additional path might also serve as the back-up route when access to the syllabary is (temporarily) unavailable. Using the assembly route, speakers need to construct syllable programs by assembling phonological segments or subsyllabic units (Laganaro, 2019; Levelt \& Wheeldon, 1994).

\section{Evidence for the retrieval of precompiled syllables}

Evidence for the retrieval of stored, precompiled syllables stem from a growing number of studies showing that high-frequency syllables are produced faster (Bürki et al., 2015; Bürki et al., 2020; Cholin et al., 2006; Laganaro \& Alario, 2006) and with greater accuracy (Laganaro \& Alario, 2006; Tremblay et al., 2016) than low-frequency syllables. These findings seem to be language-independent as effects have been found across an array of very different languages such as Dutch (Cholin et al., 2006; Levelt \& Wheeldon, 1994), English (Cholin et al., 2011; Croot et al., 2017), French (Laganaro \& Alario, 2006), Spanish (Carreiras \& Perea, 2004) and Korean (Simpson \& Kang, 2004). Syllable frequency effects can be pinpointed to the level of phonetic encoding as shown by studies opposing immediate vs. delayed naming of high- and low-frequency syllables (Cholin \& Levelt, 2009; Croot et al., 2017; Laganaro \& Alario, 2006). These studies showed that low-frequency syllables benefit more from longer preparation times in delayed naming studies compared to high-frequency syllables. When the to-be-produced item is known in advance, all levels up to articulation can contribute to the preparation effect thereby annihilating the advantage of a faster retrieval of high-frequency syllables in immediate naming studies.

It is yet unknown, whether there is a strict division of labor between those two hypothesized routes or whether the retrieval and the assembly route always run in parallel and the output is delivered by whatever route is fastest. The latter assumption of the two routes is supported by two more recent studies by Bürki et al. $(2015 ; 2020)$ that investigated eventrelated potentials (ERPs) as well as reaction times in disyllabic pseudoword production in which the initial syllables were either high-frequency, very low-frequency or novel syllables. 
3. Effects of word length and syllable frequency in adults with developmental stuttering

Novel syllables were found to be slower than high-frequency syllables in reaction times and showed more positive ERP amplitudes. Moreover, the ERPs showed different topographies (around 170ms prior to speech onset) for high-frequency and novel syllables. However, the syllable frequency effects disappeared when novel syllables were trained, suggesting that novel syllables could update to high-frequency syllables, possibly with at least a temporary storage in the syllabary (Bürki et al., 2020). This latter finding might suggest that different brain regions are involved in qualitatively different mechanisms, i.e. the retrieval route for high-frequency syllables and the assembly route for syllables from the other end of the frequency spectrum.

The current study

A systematic examination of syllable frequency effects in AWS is still pending. Given that stuttering has been associated with impairments at the interfaces between linguistic and motor planning and execution respectively, a test of syllable frequency in AWS seems essential. We hypothesize that AWS might benefit from stored syllable units even more than fluent speakers and the potential assembly of low-frequency syllables might be even more costly in an already fragile system, implicating more dysfluencies and more phonetic distortions. Thus, syllable frequency effects in AWS might be even more pronounced than in fluent speakers. The often replicated finding of word-frequency effects in AWS provides support for the assumption that frequently used items are fluency-enhancing. Importantly, when testing for syllable frequency effects, the stimulus material must be well controlled for potential confounds, particularly word and phoneme frequencies. For this reason, almost all of the above cited studies investigated syllable frequency effects by using pseudowords that resembled existing words in their respective language, i.e. were phonotactically legal strings but did not constitute actual lexical entries. Furthermore, effects of pseudoword length have been found to offer a window into the interplay between (the size of) the planning unit for articulation and the motor unit itself (Cholin et al., 2006; Cholin et al., 2011). Planning and executing multisyllabic words, speakers can start articulation already based 
3. Effects of word length and syllable frequency in adults with developmental stuttering

on the first fully encoded syllable, or they can hold articulation until more or all syllables belonging to a multisyllabic item are available. By using pseudowords, length, i.e., number of syllables, can systematically be manipulated to test for a possible interaction of syllable frequency and (pseudo-) word length.

Thus, in the current study, we set out to test whether AWS' productions of multisyllabic pseudowords are sensitive to manipulations of syllable frequency and pseudoword length: AWS were expected to exhibit higher stuttering rates and more errors when producing lowfrequency syllables as initial parts of pseudowords compared to the production of highfrequency syllables as initial parts of pseudowords. Moreover, we expected to find an effect of pseudoword length: the production of longer pseudowords (pseudowords with three or four syllables) was expected to result in higher stuttering rates with more errors than the production of shorter pseudowords (pseudowords with two syllables).

\section{Methods}

\section{Participants}

The ethical review board of the University Medical Centre Göttingen, Georg August University Göttingen, Germany approved the study and all participants provided written informed consent, according to the Declaration of Helsinki, before any study-related procedure took place. Recruitment of participants was accomplished via advertisements on university's black boards and on events of the German stuttering self-help organization (BVSS). Twenty participants per group, i.e., 20 adults with developmental stuttering (AWS) and 20 matched fluent controls (FC) were tested. Most AWS had received stuttering interventions, however for the study AWS were asked not to use any speech technique. Exclusion criteria included any speech or language disorder other than developmental stuttering, neurological impairment, drug abuse, or medications that act on the CNS. We excluded the data of one participant due to an incomplete dataset and another participant because of a medicated depression that was reported only after the experiment was 
3. Effects of word length and syllable frequency in adults with developmental stuttering

finished. Thus, we analyzed 19 AWS (4 females, mean age $27.8 \pm 10.5$ ), and 19 fluent speakers ( 3 females, mean age $26.3 \pm 8.3$ ). The groups were comparable with regard to age, sex, years of education and handedness (Oldfield, 1971) (Table 4). Speech fluency was assessed with the stuttering severity index (SSI-4; Riley, 2009) by the first author on the basis of 300 words of spontaneous speech and 300 words of reading. In the stuttering group, four participants were diagnosed with very mild stuttering, four with mild stuttering, and six with more moderate stuttering, while two participants stuttered severely, and three participants very severely. Inter-rater reliability was established by reanalyzing nine randomly chosen participants from each group by a second speech and language pathologist (N.E.N). Intraclass correlations of the SSI-4 scores yielded a good to excellent inter-rater reliability (total: ICC $=.95,95 \% \mathrm{Cl}[.87 .98]$; reading: ICC = .95, 95\% CI [.75 .99]; reading: ICC $=.92,95 \% \mathrm{Cl}[.75 .98]$; duration: $\mathrm{ICC}=.83,95 \% \mathrm{Cl}$ [.59 .93]; concomitants: ICC $=.78,95 \% \mathrm{CI}[.48 .91])$. To establish group comparability and to ensure that participants had no clinically significant deficits in cognitive functions that can influence reading behavior, seven additional subtests were administered. The WHO test of mental well-being (World Health Organization, 1998) and the Beck depression inventory (version II, Beck et al., 1996) were used for assessing mental well-being. Working memory was tested with the digit span forwards and backwards subtests of the German version of the Wechsler adult intelligence scale - fourth edition (Petermann, 2012) and the Konsonanten Trigram Test (Consonant Trigram Test, Schellig \& Schächtele, 2002). Reading performance was assessed with the one-minute word and pseudoword reading tests of the Salzburger Lese- und Rechtschreib Test-II (SLRT-II; Moll \& Landerl, 2010). Both the Digit span and the SLRT-II required the repetition of items with increasing sequence length; we therefore included stuttering severity as confounding covariate. Groups were comparable in mental well-being, reading performance, working memory and short-term memory (Table 4). In addition, we used a self-assessment of the psycho-social impact of stuttering (Overall 
3. Effects of word length and syllable frequency in adults with developmental stuttering

Assessment of the Speaker's Experience of Stuttering, OASES) (Yaruss et al., 2016).

Detailed information of assessments is provided in appendix B, Table 1 and 2.

Table 4. Demographic information of participants

\begin{tabular}{|c|c|c|c|c|}
\hline & AWS & FC & $\begin{array}{l}\text { Test-statistics } \\
(d f)\end{array}$ & $\begin{array}{l}\text { two-sided } p \text { - } \\
\text { value }\end{array}$ \\
\hline $\mathrm{n}$ & 19 & 19 & & \\
\hline Age, years & $27.8 \pm 10.5$ & $26.3 \pm 8.3$ & $183^{i}$ & .953 \\
\hline Sex ratio & $15: 4$ & $16: 3$ & $-i i$ & 1 \\
\hline Education, ranks ${ }^{a}$ & $3(1)$ & $3(0)$ & $215.5^{i}$ & .219 \\
\hline Handedness, & $88.9(15.8)$ & $87.5(29.8)$ & $149^{i}$ & .353 \\
\hline laterality quotient & & & & \\
\hline SSI-4 & $25(12.5)$ & $6(3.5)$ & - & - \\
\hline OASES & $2.1 \pm 0.7$ & - & - & - \\
\hline Onset, years & $4.8 \pm 3.0$ & - & - & - \\
\hline Well-Being index & $18(5)$ & $16(7.5)$ & $139^{i}$ & .229 \\
\hline BDI & $2(4.5)$ & $3(8)$ & $215.5^{i}$ & .309 \\
\hline SLRT-II words, n & $104.1 \pm 20.1$ & $114.2 \pm 19.9$ & $3.13(1,35)^{\mathrm{iii}}$ & .466 \\
\hline $\begin{array}{l}\text { SLRT-II } \\
\text { pseudowords, n }\end{array}$ & $64.5 \pm 13.04$ & $77.3 \pm 14.1$ & $0.21(1,35)^{\mathrm{iii}}$ & $.652^{\#}$ \\
\hline $\begin{array}{l}\text { Konsonanten } \\
\text { Trigram Test, n }\end{array}$ & $45.6 \pm 8.4$ & $48.6 \pm 5.6$ & $1.32(31.29)^{\mathrm{iii}}$ & .196 \\
\hline Digit span $f$ & $9.7 \pm 1.7$ & $10.3 \pm 1.9$ & $3.13(1,35) \mathrm{iii}$ & .085 \\
\hline Digit span b & $8.6 \pm 1.6$ & $10.1 \pm 1.9$ & $2.00(1,35)^{\mathrm{iii}}$ & .166 \\
\hline
\end{tabular}

Note. Interval/ratio -scaled variables are presented as mean \pm standard deviation. Ordinal-scaled variables are presented as median (interquartile range). "\#groups differ significantly, $p<.007$, 'MannWhitney test, iFisher's exact test, iii'ANCOVA with stuttering severity (SSI-4 total score) as confounding covariate. ${ }^{a} 1=$ high school, $2=<2$ years college, $3=2$ years of college, $4=4$ years of college. Significance level for group comparisons on cognitive functions influencing reading performance: $p=.007$ [Bonferroni corrected for multiple comparisons $.05 / 7]$. $m=$ male. $f=$ female. $n=$ number $. f=$ forward. $b=$ backward. 
3. Effects of word length and syllable frequency in adults with developmental stuttering

\section{Materials}

150 pseudoword pairs were constructed based on 250 German syllables by following phonological, phonetic and orthographic rules of the German language. The basis of these pseudoword pairs were 50 initial syllable pairs. The number of 150 pseudowords was reached by creating 50 two-syllabic, 50 three-syllabic and 50 four-syllabic pseudoword pairs (workflow in Figure 7). Care was taken that none of the syllables or the multisyllabic pseudowords constituted lexicalized forms. Summed token syllables (summed frequency of occurrence of individual syllable) were drawn from Hofmann et al. (2007) who used the phonological word form lexicon of the computer database (CEntre for LEXical Information; Baayen et al., 1996). Token frequencies of the initial syllables differentiated each pseudoword pair. Low-frequency (LF) syllables had an occurrence of less than 110 times per million (mean: 11, median: 0.5, range: 0-98), whereas syllables with a token frequency above 110 occurrences per million (mean: 2114 , median: 780 , range $=117-16467$ ) were categorized as high-frequency (HF) syllables. To reduce the influence of linguistic factors other than syllable frequency type, initial syllables of each pseudoword pair resembled each other phonetically except for the syllable nucleus (e.g. /pau/ (HF) vs. /poy/ (LF)). All pseudowords began with a bilabial or labio-dental consonant (/p/, /b/, /f/, /v/, /m/). Initial syllables had a CV $(n=12), \operatorname{CVC}(n=70), \operatorname{CCV}(n=16)$ or CCVC $(n=2)$ structure. AWS are known to stutter less in experimental settings than during daily situations (Jackson et al., 2020). To be able to elicit enough stuttering events, many trials or speech material with high phonetic complexity (Dworzynski \& Howell, 2004) need to be administered. As former studies had difficulties to generate enough stuttered trials (Mersov et al., 2017; Vanhoutte et al., 2016), we used 300 trials of overt speech production in the current study. We also ensured high phonetic complexity of stimuli by adhering to the index of phonetic complexity (IPC) (Dworzynski \& Howell, 2004) for German. 
3. Effects of word length and syllable frequency in adults with developmental stuttering

Figure 7. Workflow of pseudoword selection.

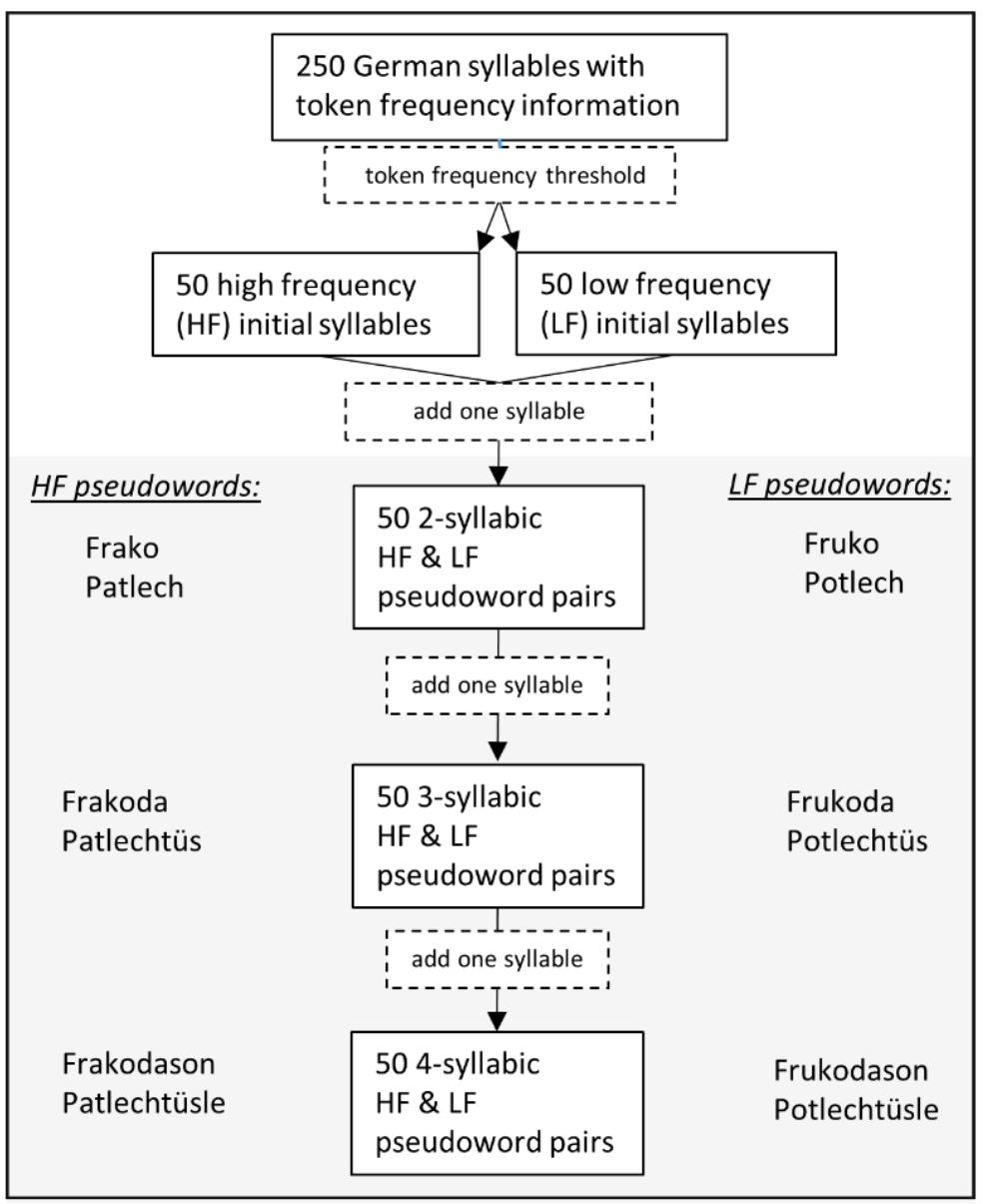

Note. $\mathrm{HF}=$ high-frequency, LF = low-frequency.

According to these authors, German speaking AWS show a higher risk to stutter if content words contain dorsal sounds (IPC factor 1), fricative, affricative or liquid sounds (IPC factor 2), if they end with a consonant (IPC factor 5), if they have more than three syllables (IPC factor 6) or if they contain consonant clusters (IPC score 7) with heterorganic articulation places (IPC score 8). Thus, all pseudowords had to score in at least four out of the six mentioned factors. Moreover, number of letters and phonemes, orthographic and phonemic bigram frequency were counterbalanced (Table 5). Pseudowords grouped by frequency type of the initial syllable did not differ in phonetic complexity (ICP) or segment length. Initial syllables grouped by syllable frequency were comparable in orthographic bigram frequency, but not in phonetic bigram frequency (Table 5). The nucleus of initial syllables grouped by 
3. Effects of word length and syllable frequency in adults with developmental stuttering

syllable frequency differed for vowel tensity but not for place of articulation (Table 5). The 300 speech stimuli were duplicated, such that each pseudoword could be read once aloud (overt condition) and once silently (covert condition). The list of pseudowords is provided in the supplement (appendix B, Table 3 and 4).

Table 5. Linguistic factors of pseudowords and initial syllables.

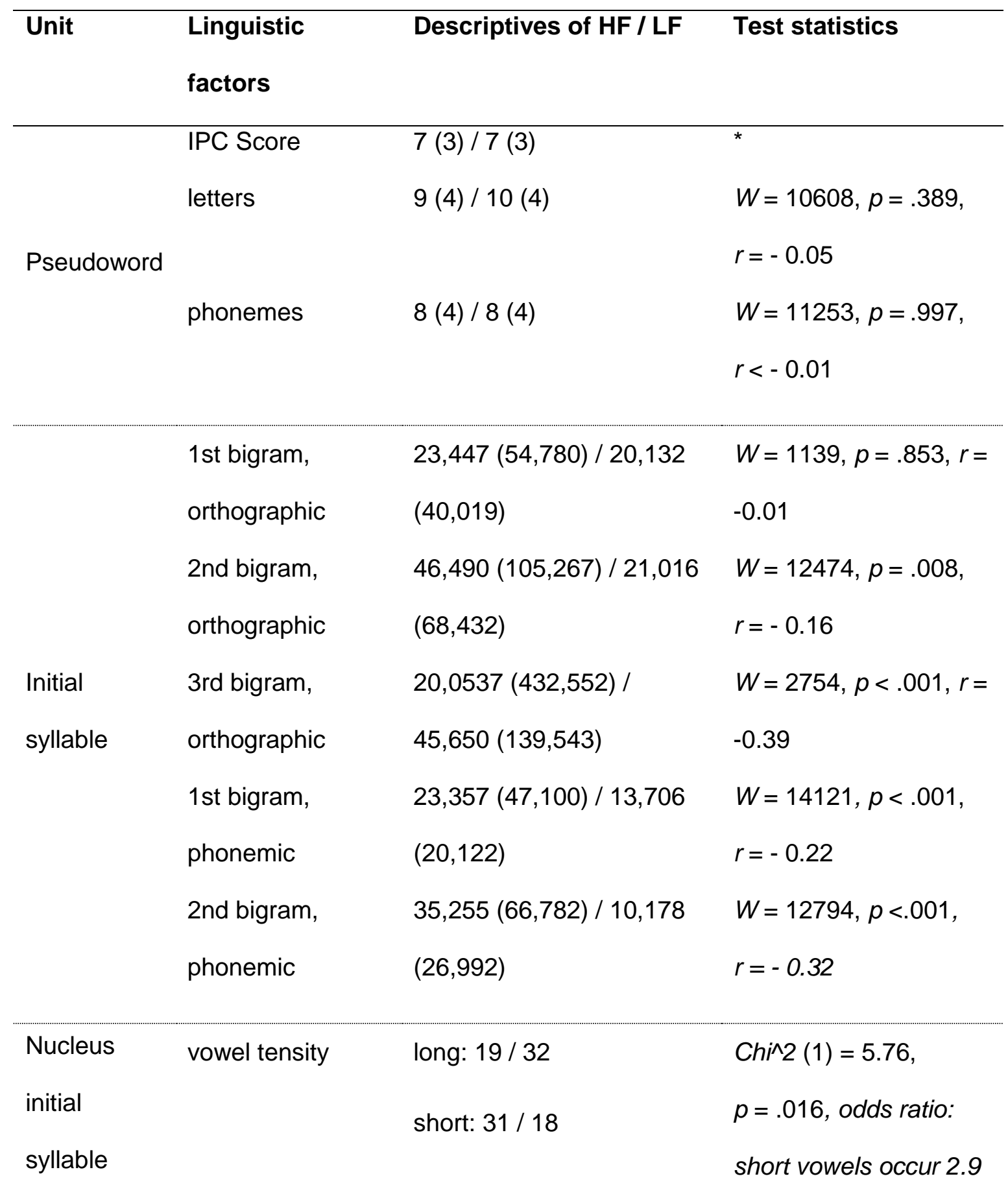


3. Effects of word length and syllable frequency in adults with developmental stuttering

times more often in HF

than in $L F$

place of

anterior: 39 / 32

$\operatorname{Chi}^{\wedge} 2(1)=1.75$,

articulation

posterior: $11 / 18$

$p=.186$, odds ratio:

posterior vowels occur

1.9 more often in LF

than in HF syllables

Note. Descriptives are given as median (IQR) or as total number. ${ }^{*}$ Please note that pseudowords grouped by frequency type had exactly the same IPC scores. Bigram frequency of phonemes/letters occurring in 3rd and/or 4th position are not reported as only few pseudowords had initial syllables with more than 4 phonemes/letters $(n<20)$. Significance level: $p=.005$ [Bonferroni corrected for multiple comparisons .05/10]. HF = high-frequency, LF = low-frequency.

\section{Design}

We used a $2 \times 2 \times 2 \times 3$ design with the factors group (AWS vs. FC), reading condition (overt vs. covert), syllable frequency (high- vs. low-frequency (first) syllables) and pseudoword length (two-syllabic, three-syllabic, four-syllabic pseudowords). The factors reading condition and pseudoword length were implemented within-pseudoword pairs and withinparticipants, the factor syllable frequency was implemented between-pseudoword pairs and within-participants. The 600 pseudowords (300 per reading condition) were divided into eight blocks, each one containing 75 pseudowords only. All blocks contained an almost equal number $( \pm 1)$ of overt and covert reading, high- and low-frequency pseudowords and two-, three-, and four-syllabic pseudowords. The sequence of blocks was pseudorandomized across the factor group. Thus, within both groups each participant read the pseudowords in a different order, but the order of pseudowords between groups was the same. In addition, for one half of the participants within each group, all pseudowords were swapped by their syllable frequency counterpart, reversing the sequence of syllable frequency. Further, the sequence of pseudowords was controlled within each block for reading condition, syllable frequency and pseudoword length. Regarding the factor reading 
3. Effects of word length and syllable frequency in adults with developmental stuttering

condition, within each block, pseudowords appeared first in the overt condition before being presented in the covert condition. In addition, the same reading condition could be consecutively repeated for up to five pseudowords. Regarding syllable frequency, either the high- or low-frequency pseudoword of a pseudoword pair was presented. A similar rule was applied for pseudoword length; pseudowords of different pseudoword length but with the same initial syllable never occurred in the same block. The covert condition served as control task of the larger EEG study and will be reported here (Korzeczek et al., in prep.).

\section{Procedure}

Participants were tested individually in a quiet room. During the experiment, participants sat in a comfortable chair at 80 centimeter distance to the computer screen wearing an EEG high-density net. The task instruction and stimuli were presented visually on the center of the screen using the experimental software PsychoPy, version 1.85.2 (Peirce, 2009; Peirce \& MacAskill, 2018).

Participants were instructed to read the visually presented pseudowords aloud when a black cross preceded them but to read the pseudowords silently when a white cross preceded them. The stimuli for task implementation of the reading condition (i.e. cross) were presented 2 sec before the appearance of the pseudowords. In addition, an inter-stimulus interval of 2.5 to $3.5 \mathrm{sec}$ after the presentation of the pseudoword and before the next task implementation separated trials. Pseudowords in the overt reading condition were presented for $6 \mathrm{sec}$, whereas the presentation of pseudowords was reduced to $2 \mathrm{sec}$ in the covert condition. After each block a planned pause, which allowed participants for positional readjustment or to drink and further assessments, was implemented. Every participant followed the same sequence of pause durations and test assessments.

As a self-initiated speech preparation was used, participants were instructed to wait for an internal urge to speak before overtly reading the presented pseudoword (McArdle et al., 2009). Because prosodic unknown pseudowords were used, participants were asked to pretend as if reading a German word. In addition, participants were instructed not to use 
3. Effects of word length and syllable frequency in adults with developmental stuttering

any fluency enhancing technique, not to suppress stuttering events or to correct themselves. Ten practice trials, in which participants were informed on their response correctness, were conducted before starting the experiment.

\section{Data preprocessing}

During the experiment, participants were videotaped. Two speech and language pathologists, K.H. and J.W., transcribed and evaluated speech samples of all participants for stuttering events, errors and their syllable position using ELAN, version 5.8 (Max Planck Institute for Psycholinguistics, Nijmegen, 2018). Inter-rater reliability was maintained by comparing the evaluation of four randomly chosen participants (two per group). Stuttering events and response accuracy were recognized with an inter-rater reliability of 0.68 and respectively 0.65 as measured with Cronbach's alpha. In addition, the first author (AK) checked all transcriptions for consistency. Some orthographic pseudowords allowed different phonetic implementations, as participants did not listen to a correct pronunciation of the pseudowords. Ambiguous realizations of a pseudoword included incorrect syllabification (e.g. /ge: us/ instead of /goys/) and incorrect vowel tensity of initial syllables (e.g. /vu:k/instead of /vvk/). Such realizations were marked as unclear errors. Pseudowords with unclear errors as well as trials with missing verbal responses were excluded from further statistical analysis. To keep the stimuli material balanced, the categorical frequency partners of these discarded pseudowords were excluded as well.

\section{Dependent variables}

Two dependent variables were measured: (1) speech fluency, i.e., stuttering events and (2) response accuracy, i.e. errors. Responses were either marked for being correct or for containing a stuttering event or an error.

Stuttering events in AWS were registered using the SSI-criteria (Riley, 2009). These consist of sound and syllable repetitions or prolongations as well as blocks with visible speech 
3. Effects of word length and syllable frequency in adults with developmental stuttering

effort. Sound and syllable repetitions that were preceded by an error were not counted as stuttering symptoms but as self-corrections.

Errors in both groups were defined as additions, elisions, substitutions and metathesis of consonants and vowels. As in Tremblay et al. (2016), substitutions of vowels in medial or final syllables addressing their tensity or length (e.g. /o/ vs. /o/, /u/ vs. / / or /a/ vs. /a:/) were not considered as errors as these might be viewed as correct adaptation due to a change in word stress.

Multiple occurrences of one single variable within the same pseudoword were counted as one event (Sasisekaran \& Weathers, 2019; Yaruss, 1999). We excluded pseudowords and their pairs containing an error and stuttering event (AWS: $2.12 \%, \mathrm{FC}: 0.34 \%$ ) from statistical analyses (Coalson \& Byrd, 2017).

\section{Statistical analysis}

To investigate the influence of syllable frequency on stuttering events and errors we calculated two main and two additional Generalized Linear Mixed Models (GLMM, Baayen et al., 2008), with binomial error structure and logit link function. The two main models investigated the influence of syllable frequency on stuttering events or errors over all syllable positions. In addition, we analyzed only stuttering events or errors that occurred at the initial syllable position.

For all models, we included the z-transformed covariate of pseudoword length, the factor syllable frequency and their interaction as within-subject fixed effects into the models. In addition, for modelling errors, we included the between-subjects factor group and its interaction with syllable frequency and pseudoword length as well as their overall three-way interaction as fixed effects. To account for speech performance, we included stuttering severity (summed SSI-4 scores of reading and spontaneous speech) to the models of stuttering probability and reading fluency performance (number of read pseudowords, SLRT-II) to the models of error probability as z-transformed covariates. Several linguistic factors revealed differences between pseudoword pairs (see Table 5). To check whether 
3. Effects of word length and syllable frequency in adults with developmental stuttering

these factors influenced the variance of our data, we added first and second phonemic bigram frequency as wells as vowel length separately as fixed effects to the full models. Factors that significantly improved the model as indicated by likelihood ratio tests ( $R$ function anova with argument test set to "Chisq") were kept (appendix B, Table 5). We included all possible random effects and slopes, to keep type I error at the nominal level of $5 \%$ (Barr et al., 2013). Possible random terms were determined by following the procedure of Bates et al. (2018). We first fitted the maximal model including all possible random effect components and then iteratively reduced the model from random terms that were not supported by the data. Reduction steps were based on principal component analyses using the function rePCA and likelihood ratio tests (Bates et al., 2018).

Model assumptions were checked by inspecting visually the distribution of residuals, by assessing model stability (appendix B, Table 6 and 7) and by ruling out collinearity with the Variance inflation factors being beyond 1.5 (Field et al., 2013). The variance inflation factor was derived by the function vif of the R-package car (Fox \& Weisberg, 2019), applied to a standard linear model excluding the random effects. All models met the assumption criteria. As an overall test of the effect of the fixed effects and their interactions we compared the full model with a null model lacking the fixed effects (Field et al., 2013) using a likelihood ratio test. The statistical significance of fixed effects was tested based on likelihood ratio tests, comparing the full model with the respective reduced models ( $\mathrm{R}$ function drop1). Reduced models comprised the same random effect structure as the full model. The models were fitted in $\mathrm{R}$ (version 3.6.2, $\mathrm{R}$ Core Team, 2019) using the function g/mer of the $\mathrm{R}$ package Ime4 (version 1.1-21) (Bates et al., 2015). Confidence intervals were derived using the function bootMer of the package Ime4 with 1,000 parametric bootstraps.

\section{Results}

Premise for subsequent analyses: Groups are comparable with regard to unclear errors To ensure that the discarded unclear errors did not influence our analyses on syllable frequency effects, we run a factorial two-way ANOVA with Group as between-subjects 
3. Effects of word length and syllable frequency in adults with developmental stuttering

factor and syllable frequency as within-subjects factor. On average, AWS produced 29.32 $(S D=12.12)$ unclear errors in high-frequency and $31.16(S D=12.06)$ unclear errors in lowfrequency pseudowords. FC produced on average $25.11(S D=6.44)$ unclear errors in highfrequency and $27.95(S D=5.73)$ unclear errors in low-frequency pseudowords. Results showed no main effect of group, $F(1,72)=2.86, p=.09$, $\omega 2=0.92$, no effect of syllable frequency, $F(1,72)=1.14, p=.29, \omega 2=0.25$, and no interaction, $F(1,72)=0.05, p=.82$, $\omega 2=-1.17$. Thus, groups did not differ by unclear reading errors, nor did unclear errors affect a specific frequency category. For statistical hypotheses testing 7,570 (FC: 3,958; AWS: 3,612) pseudowords remained.

Pseudoword length but not syllable frequency influences stuttering probability when considering all syllable positions

The first generalized linear mixed-effects model involved stuttering events as the dependent variable and syllable frequency (high- and low-frequency of initial syllables), the ztransformed covariate of pseudoword length and stuttering severity and the two-way interaction of pseudoword length and syllable frequency as independent variables. In addition, the z-transformed covariates of Bigram transition token frequency contributed significantly to the model (appendix B, Table 5) and was added as further covariates to the full model. Overall, 2,772 items were included in the analysis (for descriptive statistics see Table 6, for details see appendix B, Table 8).

Table 6. Descriptive statistics of stuttered events over all syllable positions in AWS.

\begin{tabular}{|c|c|c|c|c|c|c|}
\hline \multirow[t]{2}{*}{ Stuttered events } & \multicolumn{2}{|c|}{ two- syllabic } & \multicolumn{4}{|c|}{ three- syllabic four- syllabic } \\
\hline & HF & LF & HF & LF & HF & LF \\
\hline total responses $(n)$ & 526 & 526 & 471 & 471 & 389 & 389 \\
\hline stuttered events (n) & 85 & 74 & 112 & 96 & 118 & 114 \\
\hline stuttered events (mean) & 0.162 & 0.141 & 0.238 & 0.204 & 0.303 & 0.293 \\
\hline stuttered events (SD) & 0.368 & 0.348 & 0.426 & 0.403 & 0.460 & 0.456 \\
\hline
\end{tabular}


3. Effects of word length and syllable frequency in adults with developmental stuttering

The model was significant compared to the null model $\left(x^{2}(7)=104.66, p<.001\right)$. The occurrence of stuttered events was significantly influenced by pseudoword length $\left(\mathrm{X}^{2}(1)=83.25, \mathrm{p}<.001, \beta=0.53, \mathrm{SE}=0.06,95 \% \mathrm{Cl}[0.42,0.66]\right)$ (Figure 8A, $\left.\mathrm{B}\right)$. In addition, stuttering severity predicted the occurrence of stuttered events significantly $\left(X^{2}(1)=11.8\right.$, $p<.001, \beta=1.32, \mathrm{SE}=0.3,95 \% \mathrm{Cl}[0.74,2])$. The more severe the stuttering of a participant, the more stuttering events occurred during the experiment (Figure 8A, C). Neither initial syllable frequency nor phonemic transition bigram frequency were significant (syllable: $x^{2}(1)=1.61, p=.204$, bigram: $\left.x^{2}(1)=3.45, p=.063\right)$. (Figure 8A).

Figure 8. Data for each factor contributing to stuttering events over all syllable positions.

A

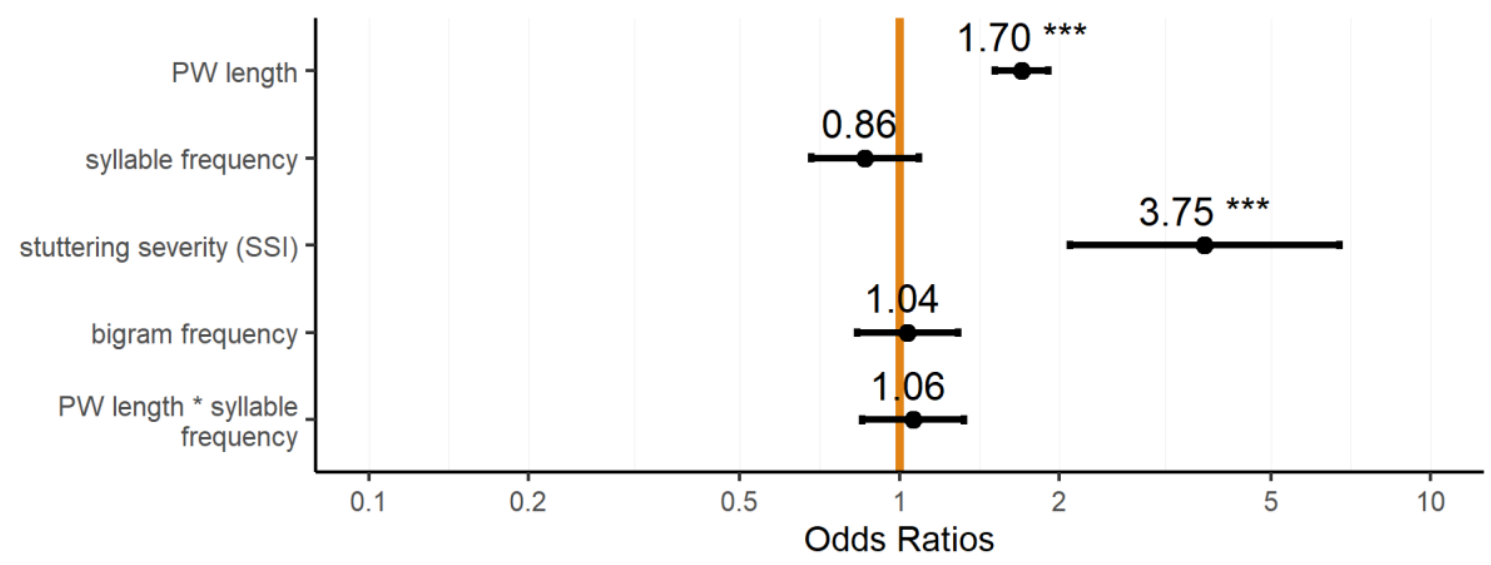

B

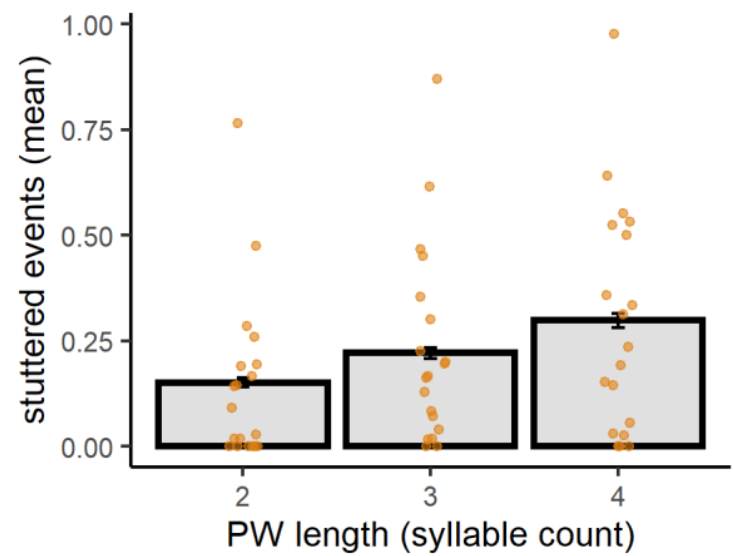

C

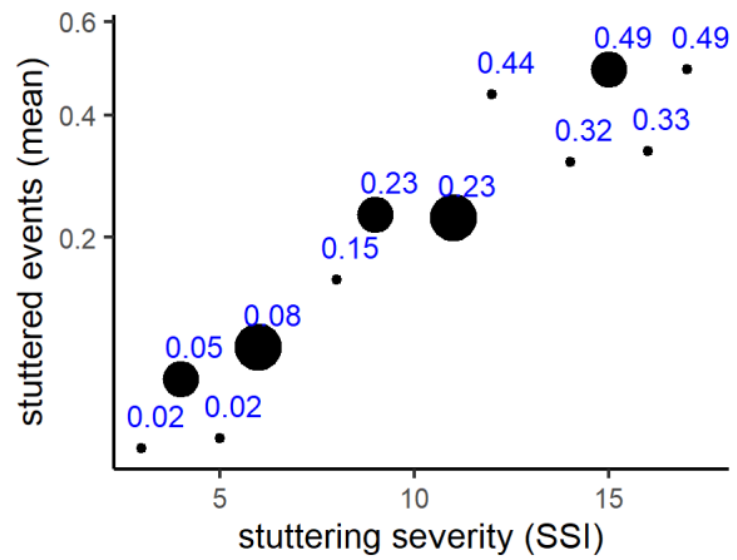

Note. A. Stuttering probability as indicated by Odds ratios was significantly influenced by pseudoword (PW) length (increase in syllables) and stuttering severity. Whiskers indicate confidence intervals at 0.95. Contrasts of syllable frequency were set to -0.5 (LF) vs. 0.5 (HF). B. Main effect of pseudoword length: Bar plots, represent means of stuttered events for two-, three- and four-syllabic pseudowords. Points represent individual means of participants. Error bars represent standard errors. C. Main effect of stuttering severity: Scatterplot depicts mean of stuttered events according to stuttering severity, 
3. Effects of word length and syllable frequency in adults with developmental stuttering

point size depicts the number of participants diagnosed with the respective stuttering severity score (small point: one participant, largest point: three participants).

Trend of syllable frequency effect on stuttering probability in first syllable position

Next, we investigated the effect of syllable frequency on the probability to stutter on syllables in initial word positions. In addition to the main fixed effects, vowel length, which contributed significantly to the model (appendix B, 5), was added to the full model. Overall, 2,458 pseudowords were included in the analysis (for descriptive statistics see Table 7)

Table 7. Descriptive statistics of stuttered events in initial syllable positions in AWS.

\begin{tabular}{llllllll}
\hline Stuttered events & \multicolumn{3}{l}{ two- syllabic } & \multicolumn{3}{l}{ three- syllabic } & four- syllabic \\
& HF & LF & HF & LF & HF & LF \\
& & & & & & \\
\hline total responses (n) & 510 & 510 & 410 & 410 & 309 & 309 \\
stuttered events (n) & 76 & 62 & 70 & 53 & 57 & 56 \\
stuttered events (mean) & 0.149 & 0.122 & 0.171 & 0.129 & 0.184 & 0.181 \\
stuttered events (SD) & 0.356 & 0.327 & 0.377 & 0.336 & 0.388 & 0.386
\end{tabular}

$\overline{\text { Note. Due to the binomial distribution the mean } 100 \text { represents the percentage of }}$ stuttered events. HF = high-frequency, LF = low-frequency.

The model was significant compared to the null model $\left(x^{2}(5)=30.9, p<.001\right)$. As in the main model, the occurrence of stuttered events was significantly influenced by pseudoword length $\left(x^{2}(1)=13.91, p<.001, \beta=0.29, S E=0.08,95 \% \mathrm{Cl}[0.13,0.45]\right)$ and stuttering severity $\left(X^{2}(1)=15.21, p<.001, \beta=1.87, S E=0.42,95 \% \mathrm{Cl}[1.06,2.81]\right)$ (Figure 9A). Neither syllable frequency nor vowel length were statistically significant (syllable frequency: $X^{2}(1)=1.77, p=.183$, vowel length: $\left.X^{2}(1)=0.26, p=.609\right)$. However, contrary to our hypothesis of a syllable frequency effect, descriptively, AWS showed higher stuttering rates on high-frequency pseudowords (Figure 9A, B). 
3. Effects of word length and syllable frequency in adults with developmental stuttering

Figure 9. Data for each factor contributing to stuttered events in initial syllable position.

A

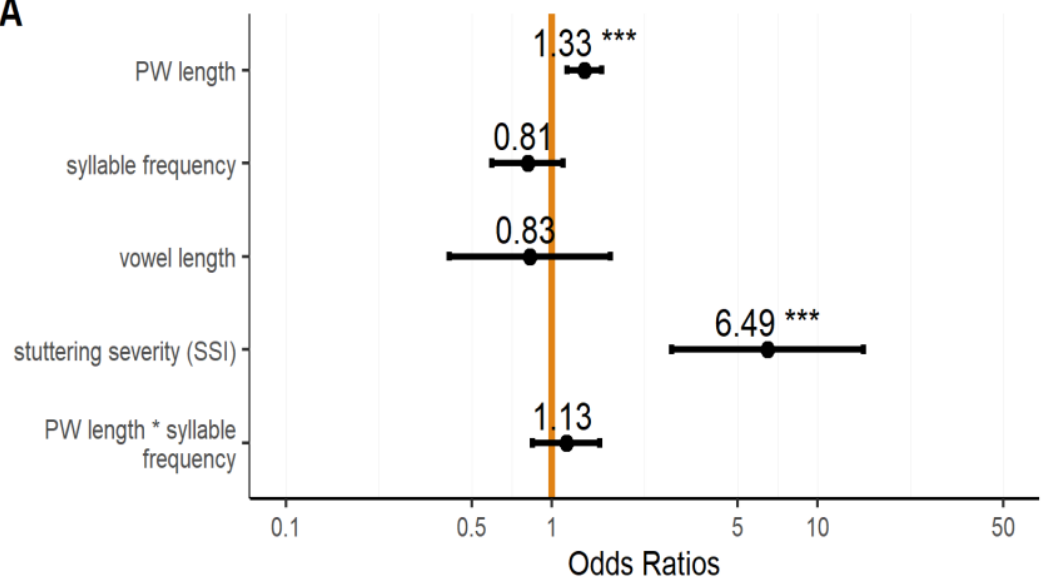

B

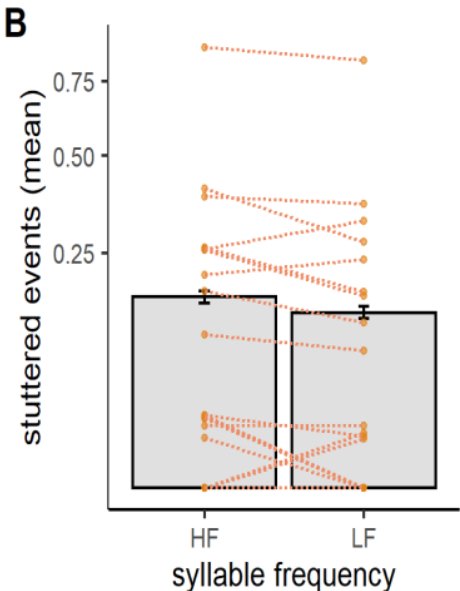

Note. A. Stuttering probability as indicated by Odds ratios was significantly influenced by pseudoword (PW) length (increase in syllables) and stuttering severity. Contrasts of syllable frequency were set to -0.5 (LF) vs. 0.5 (HF). B. Descriptive trend of syllable frequency: Bar plots represent means of stuttered events for high- (HF) and low- (LF) frequency pseudowords. Points represent individual means of participants. Error bars represent standard errors.

Pseudoword length but not syllable frequency influences response accuracy when considering all syllable positions

For modelling errors, we included syllable frequency (high- and low-frequency initial syllables), group (AWS and FC) and the $z$ transformed covariates of pseudoword length and reading fluency performance (SLRT-II pseudoword score) and the three-way interaction between group, frequency and length. Fixed effects of vowel length or token frequency of phonemic bigrams did not improve the model, all $p>.05$. Overall, 6,170 items were included in the analysis (for descriptive statistics see Table 8). 
3. Effects of word length and syllable frequency in adults with developmental stuttering

Table 8. Descriptive statistics of errors over all syllable positions per group.

\begin{tabular}{|c|c|c|c|c|c|c|c|}
\hline \multirow{2}{*}{ Group } & \multirow{2}{*}{ Errors } & \multicolumn{2}{|c|}{ two- syllabic } & \multicolumn{2}{|c|}{ three- syllabic } & \multicolumn{2}{|c|}{ four- syllabic } \\
\hline & & HF & LF & HF & LF & HF & LF \\
\hline \multirow{4}{*}{$\mathrm{FC}$} & total responses $(n)$ & 664 & 664 & 634 & 634 & 579 & 579 \\
\hline & errors (n) & 36 & 39 & 73 & 63 & 89 & 105 \\
\hline & errors (mean) & 0.054 & 0.059 & 0.115 & 0.099 & 0.154 & 0.181 \\
\hline & errors (SD) & 0.227 & 0.235 & 0.319 & 0.299 & 0.361 & 0.386 \\
\hline \multirow{4}{*}{ AWS } & total responses $(n)$ & 471 & 471 & 405 & 405 & 332 & 332 \\
\hline & errors (n) & 34 & 33 & 47 & 44 & 53 & 67 \\
\hline & errors (mean) & 0.072 & 0.070 & 0.116 & 0.109 & 0.160 & 0.202 \\
\hline & errors (SD) & 0.259 & 0.256 & 0.321 & 0.312 & 0.367 & 0.402 \\
\hline
\end{tabular}

The model was significant compared to the null model $\left(x^{2}(8)=47.59, p<.001\right)$. The occurrence of errors was significantly influenced by pseudoword length $\left(x^{2}(1)=35.99\right.$, $p<.001, \beta=0.55, \mathrm{SE}=0.07,95 \% \mathrm{Cl}[0.4,0.69])$. Participants produced more errors on three- and four- syllabic than on two-syllabic pseudowords (Figure 10A, B). In addition, reading performance predicted the occurrence of errors significantly $\left(x^{2}(1)=8.31, p=.004\right.$, $\beta=-0.31, \mathrm{SE}=0.1,95 \% \mathrm{Cl}[-0.52,-0.11])$. Indicating that participants with low score in the SLRT-II pseudoword task made more errors (Figure 10A, C). Neither initial syllable frequency nor group or the three-way interaction were statistically significant (Figure 10A). 
3. Effects of word length and syllable frequency in adults with developmental stuttering

Figure 10. Data for each factor contributing to errors overall syllable positions.

A

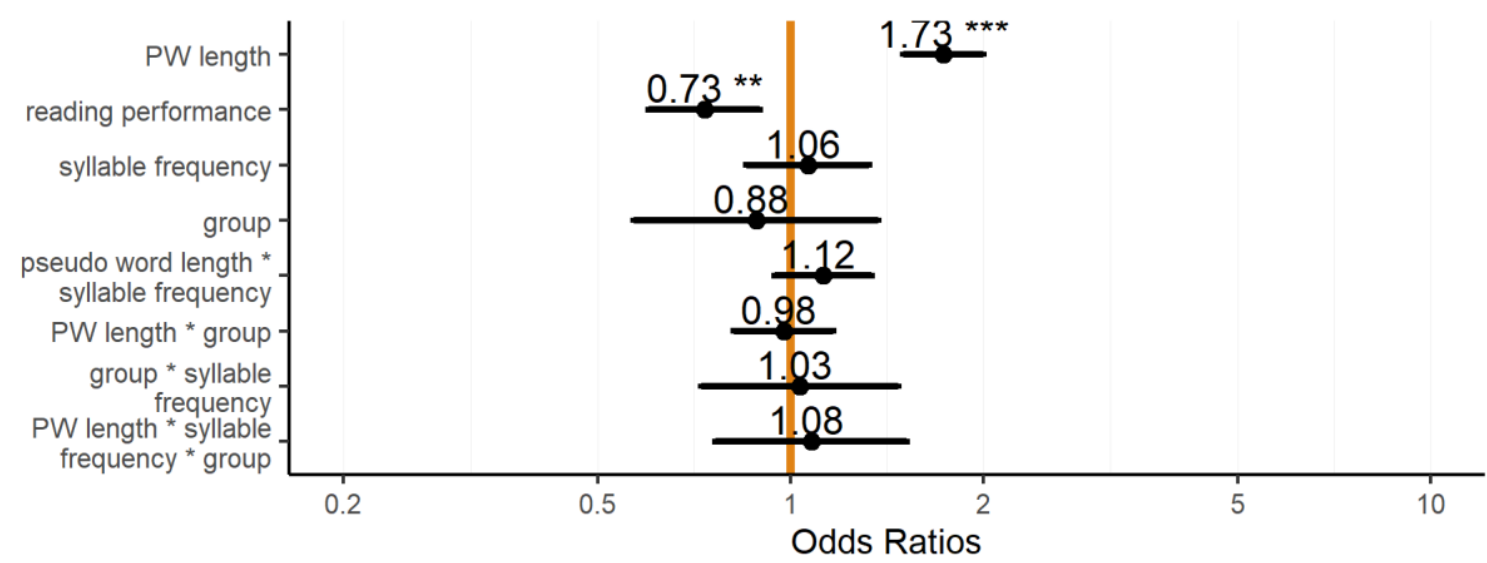

B

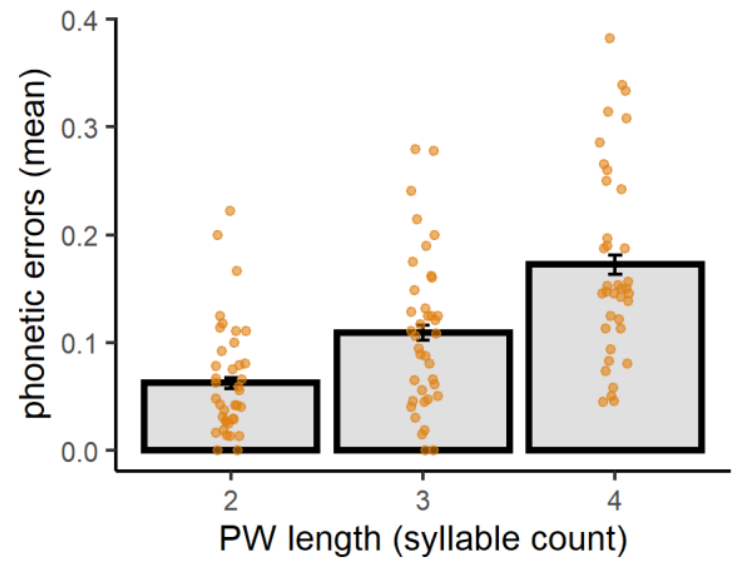

C

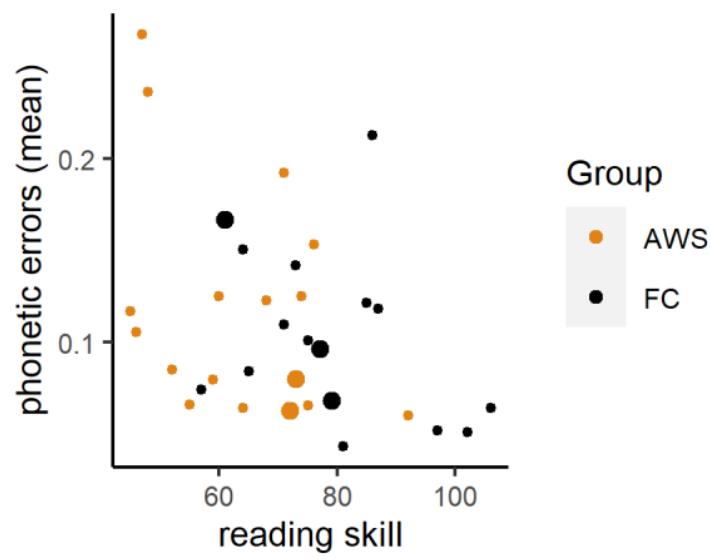

Note. A. Probability of errors as indicated by Odds ratios was significantly influenced by pseudoword (PW) length and reading skill. B. Main effect of pseudoword length: Bar plots represent Group means of errors for two-, three-, and four-syllabic pseudowords. Points represent individual means of participants. Error bars represent standard errors. C. Main effect of reading skill: Means of errors plotted against score in reading skill (score in SLRT-Il pseudoword). Point size depicts the number of participants that received the respective reading skill, small point: one participant, largest point: two participants.

AWS are less accurate on high-frequency initial syllables in two- and three-syllabic pseudowords than FC

For modelling errors in initial syllable position as response variable, we included syllable frequency, group and the z-transformed covariates of pseudoword length and reading performance and all possible interactions between group, frequency and length. Vowel length or token frequency of phonemic bigrams did not improve the model, all $p>.05$. Overall, 5,404 items were included in the analysis (for descriptive statistics see Table 9). 
3. Effects of word length and syllable frequency in adults with developmental stuttering

Table 9. Descriptive statistics of errors in initial syllable positions per group.

\begin{tabular}{|c|c|c|c|c|c|c|c|}
\hline \multirow{2}{*}{ Group } & \multirow{2}{*}{ Responses in } & \multicolumn{2}{|c|}{ two- syllabic } & \multicolumn{2}{|c|}{ three- syllabic } & \multicolumn{2}{|c|}{ four- syllabic } \\
\hline & & HF & LF & HF & LF & HF & LF \\
\hline \multirow{4}{*}{ FC } & responses $(n)$ & 636 & 636 & 548 & 548 & 466 & 466 \\
\hline & errors (n) & 19 & 21 & 13 & 15 & 15 & 24 \\
\hline & errors (mean) & 0.030 & 0.033 & 0.024 & 0.027 & 0.032 & 0.052 \\
\hline & errors (SD) & 0.170 & 0.179 & 0.152 & 0.163 & 0.177 & 0.221 \\
\hline \multirow{4}{*}{ AWS } & responses $(n)$ & 440 & 440 & 352 & 352 & 260 & 260 \\
\hline & errors (n) & 18 & 12 & 16 & 10 & 10 & 17 \\
\hline & errors (mean) & 0.041 & 0.027 & 0.045 & 0.028 & 0.038 & 0.065 \\
\hline & errors (SD) & 0.198 & 0.163 & 0.209 & 0.166 & 0.193 & 0.248 \\
\hline
\end{tabular}

Note. $\mathrm{HF}=$ high-frequency, LF = low-frequency.

The model was highly significant compared to the null model $\left(x^{2}(8)=30.12, p<.001\right)$. The model revealed that response errors were significantly influenced by the interaction between syllable frequency and group $\left(X^{2}(1)=10.02, p=.002, \beta=-1.38\right.$, SE $=0.45$, $95 \% \mathrm{CI}[-2.44,-0.59])$. Contrary to our hypothesis, AWS made more errors on pseudowords with initial high-frequency syllable compared to pseudowords with initial low-frequency syllable, whereas FC showed the opposite pattern (Figure 11A, B). Furthermore, we found a significant interaction between initial syllable frequency and pseudoword length with $\left(x^{2}(1)=4.5, p=.002, \beta=0.34, S E=0.16,95 \% \mathrm{Cl}[0.03,0.66]\right)$. Figure $11 \mathrm{C}$ shows that contrary to two- and three-syllabic words, low-frequency four-syllabic pseudowords elicited considerably more errors than high-frequency four-syllabic pseudowords (Figure 11A, C). This interaction, should however be regarded with caution. Although, the three-way interaction did not become significant, descriptive statistics reveal (Table 9) that the higher error rate for high-frequency two-syllabic and three-syllabic pseudowords compared to their low-frequency counterparts was evident in AWS. As in the previous model, pseudoword 
3. Effects of word length and syllable frequency in adults with developmental stuttering

length $\left(x^{2}(1)=6.49, p=.011, \beta=0.21, S E=0.08,95 \% \mathrm{Cl}[0.04,0.37]\right)$ and reading performance $\left(X^{2}(1)=7.96, p=.005, \beta=-0.46, S E=0.15,95 \% \mathrm{CI}[-0.78,-.15]\right)$ predicted the occurrence of errors.

Figure 11. Data for each factor contributing to errors in initial syllable position.

A

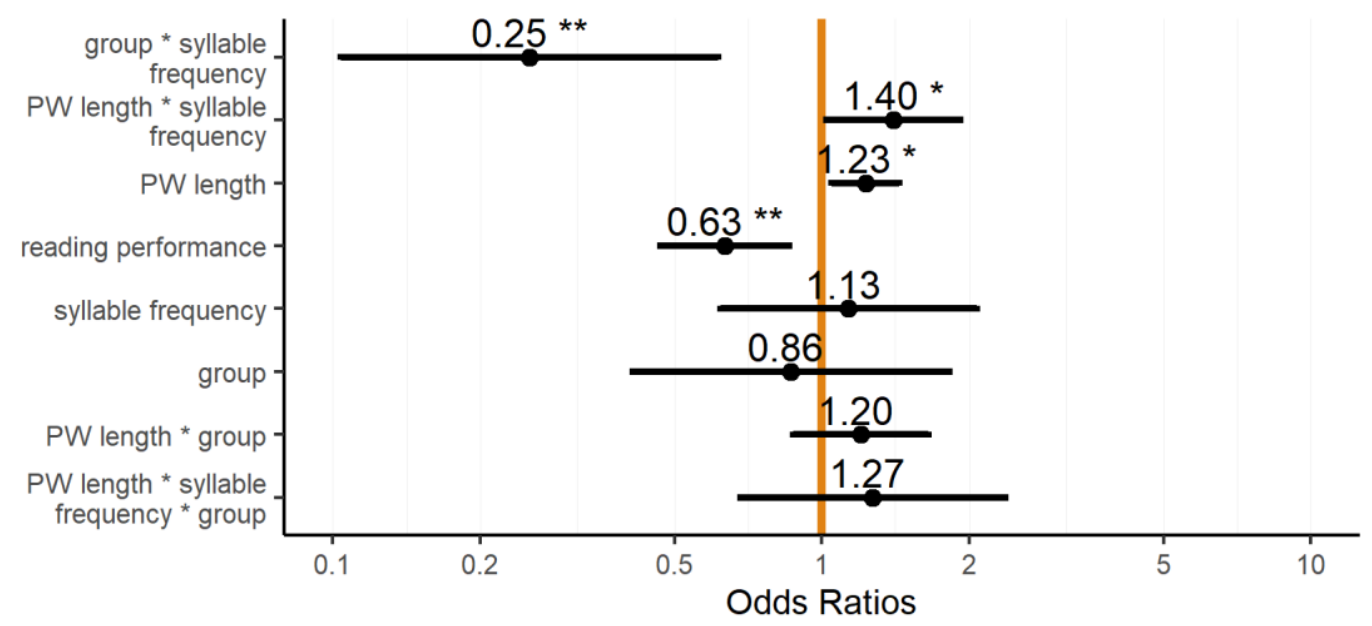

B

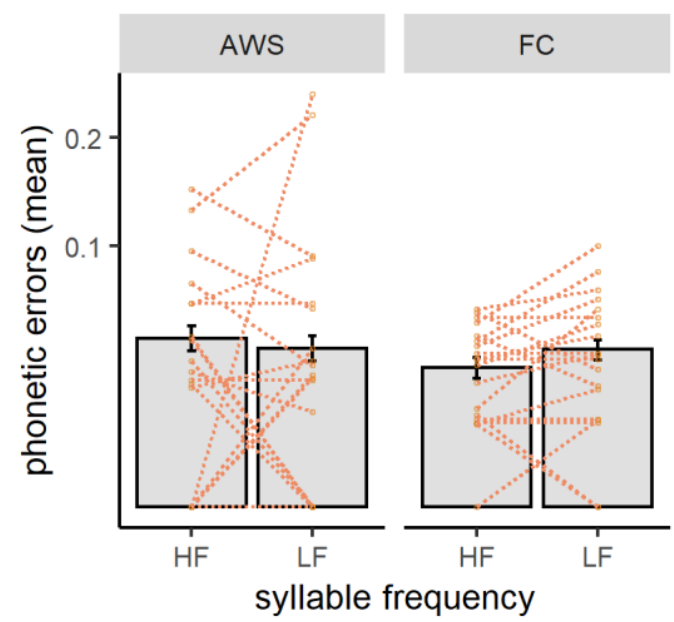

C

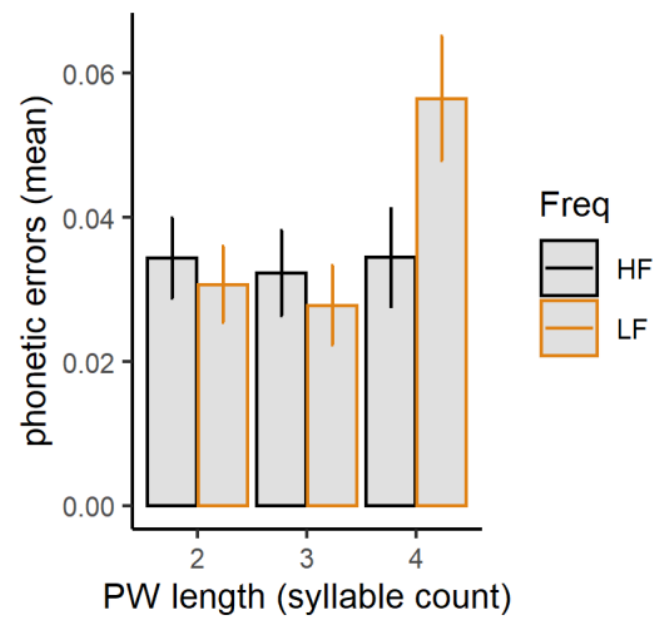

Note. A. Probability of errors in initial syllable position as indicated by Odds ratios was significantly influenced by the interaction between syllable frequency and group, by syllable frequency and pseudoword (PW) length, by pseudoword length and by reading skill. B. Bar plots represent group means of errors for high- (HF) and low- (LF) frequency syllables. C. Bar plots represent mean of two- , three-, and four-syllabic pseudowords separated by syllable frequency. Error bars represent standard errors. 
3. Effects of word length and syllable frequency in adults with developmental stuttering

\section{Discussion}

This study examined whether speech fluency and accuracy were influenced by the frequency of initial syllables and by the number of (adjacent) syllables in pseudowords with increasing length (two-, three-, and four-syllables). In light of the syllable frequency effects in fluent speakers (see section "Evidence for the retrieval of precompiled units"), we expected high-frequency syllables to be uttered more fluently and with greater accuracy compared to low-frequency syllables also in our non-fluent participants. However, syllable frequency did not influence our first dependent variable speech fluency of initial syllables and pseudowords as a whole of AWS. In fact, when analyzing stuttering events in initial syllable position only, there was a trend in the opposite direction: $63 \%$ of our participants stuttered more on high- than on low-frequency first syllables. This opposite response pattern of syllable frequency became even more prominent when analyzing our second dependent variable response accuracy of the initial first syllable of pseudowords. Here, as indicated by a significant group by syllable frequency interaction, AWS produced more errors on pseudowords containing high-frequency first syllables compared to those pseudowords with low-frequency first syllables. FC showed the opposite trend. Although the three-way interaction between group, syllable frequency and pseudoword length was not statistically significant, AWS' tendency to produce more errors on high-frequency syllables compared to low-frequency syllables included another interesting finding: in short pseudowords, i.e. two- and three-syllabic, more errors were found for with high-frequency first syllables compared to their low-frequency counterparts. However, this pattern was reversed for long pseudowords. In four-syllabic pseudowords with low-frequency first syllables, a much higher error probability occurred than in the equivalent items with high-frequency first syllables. In addition, the main effect of pseudoword length showed a robust effect for both dependent variables, confirming our hypothesis that the number of stuttering events and response errors would increase with pseudoword length. In the following sections, we will first address 
3. Effects of word length and syllable frequency in adults with developmental stuttering

the question of what might have led to the lack of the expected syllable frequency effects in AWS.

\section{Syllable frequency}

The dual-route account offers an explanation for the advantage of high-frequency syllables over low-frequency syllables in fluent speakers. The corner stone for a store hosting oftenused motor programs is laid in early stages of speech acquisition when syllables are learned by imitation and repetition of speech sounds that are heard and recurrently adapted via sensorimotor links (Levelt et al., 1999; see Kearney \& Guenther, 2019, for a recent sketch of such a framework). For AWS, we had predicted syllable frequency effects to be even stronger with the difference between the two mechanisms being more pronounced in AWS. In a fragile system, we had expected, the reliance on early acquired, high-frequency syllables would be even greater and hence the frequency effect to be more pronounced. Especially since the assembly process that is thought to be already more resource-taxing in fluent speakers would be even more arduous and error-prone in AWS.

However, the above reported results showed a different pattern. One account for these unexpected results might lie in the assumption that the dual route mechanism or parts of it operate differently in AWS. With respect to the retrieval route, AWS may not acquire and store motor programs in the same way as fluent speakers do, which could lead to a lower efficiency in phonetic encoding. Accumulating evidence points towards impaired sensorimotor learning processes in AWS in speech as well as non-speech tasks (e.g. Kim et al., 2020; Namasivayam \& van Lieshout, 2008; Smith et al., 2010; Smith \& Weber, 2017; Smits-Bandstra, De Nil, \& Rochon, 2006; Smits-Bandstra, De Nil, \& Saint-Cyr, 2006). The idea of inadequately acquired motor programs that may entail a number of adverse repercussions for speech planning in AWS has not been considered within cognitive accounts of stuttering but it might offer a suitable explanation for the current results (but see Max, Guenther et al., 2004 for a similar account in a model of motor control). Within the dual-route account different scenarios seem tangible: (i) a program that does not qualify as 
3. Effects of word length and syllable frequency in adults with developmental stuttering

a stable motor entity takes longer to be selected from a pool of neighboring syllables and might necessitate more intense phonetic fine tuning, (ii) instead of the (fragile) target syllable that cannot overcome activation threshold in a timely fashion, eventually, a close competitor gets selected. The erroneous selection of a stored syllable might clash with the output delivered by the assembly route. Resolving this conflict will (also) delay further processing. Either way, only when the decision for the target syllable is completed, the syllable boundary marking the end of the current syllable during syllabification procedures, the next syllable can be sent into further encoding, until then, processing of subsequent syllables is put on hold.

It might also be the case that the stuttering system decides, due to persistent problems with accessing motor programs in the syllabary, to abandon and bypass the retrieval route and give preference to the assembly route instead. If this were the case, high-frequency syllables will no longer have an advantage over low-frequency syllables since now all syllables will be built from scratch. Our finding of a group by syllable frequency interaction might be more in line with the assumption of less stable high-frequency syllables.

Taken together, under the hypothesis of fragile motor programs in AWS, all scenarios imply that high-frequency syllables will not have an advantage over low-frequency ones but might inflict more errors due to erroneously selected syllables or compensatory repair processes. The stronger interconnectedness of the individual motor subparts within high-frequency syllables that facilitate production in fluent speakers might have a detrimental impact on AWS speech as it might force larger motor chunk upon a more fragile/vulnerable system that actually prefers smaller chunks (for arguments regarding insufficient motor control in AWS see Namasivayam \& van Lieshout, 2011; Smith \& Weber, 2017). It should be noted though, that, as variable as motor planning is in AW at times, the retrieval of precompiled larger motor chunks albeit fragile or only loosely interconnected, these programs might occasionally be judged as "good" enough and will guide the subsequent processes to a successful articulatory execution. The variability with which AWS can sometimes produce 
3. Effects of word length and syllable frequency in adults with developmental stuttering

certain motor programs while they cannot at other times has made it difficult to understand which phonetic gestures or combinations thereof might evoke stuttering more than others. An attempt that has gained considerable impetus in that regard is the work by Howell and colleagues (Al-Tamimi et al., 2013; Dworzynski \& Howell, 2004; Howell et al., 2006; Howell \& Au-Yeung, 2007) showing that higher phonetic complexity (for IPC scores of the current materials see Method section) can be associated with higher stuttering rates. However, a clear pattern that can reliably predict stutter occurrences providing insights into underlying compensatory strategies, which could also successfully be applied in treatment approaches, has not yet emerged. In the current study, error analyses did not show any general or individual patterns among our AWS group. Our materials were carefully controlled for many potentially confounding factors but high- and low-frequency studies differed with respect to bigram frequency and vowel length. To account for any potential influence (e.g. Coalson et al., 2018; Kalveram, 2001), we included these factors in our analyses. Although, none of these factors reached significance, in future studies these factors should be targeted directly to understand AWS's error pattern and potential compensatory strategies.

\section{Pseudoword length}

With respect to pseudoword length, the results turned out as predicted: there was a clear length effect in stuttering rate and in error rate: longer pseudowords were less fluent and less accurate while shorter words were more fluent and more accurate. This finding is in line with findings with regard to speed and/or accuracy (e.g. fluent speakers: Damian \& Dumay, 2007; Meyer et al., 2003; Windsor et al., 2010; AWS: Byrd et al., 2012; Byrd et al., 2015; Sasisekaran \& Weisberg, 2014; speakers with neurological language disorders: Croot et al., 1998; Nickels \& Howard, 2004), stuttering disfluencies (e.g., in adults: Max et al., 2019; in children: Logan \& Conture, 1995; Sasisekaran \& Weathers, 2019).

Within the psycholinguistic literature, effects of word length have been vividly debated, most notably by (Alario et al., 2002a, 2002b; Levelt, 2002). The debate is closely related to the 
3. Effects of word length and syllable frequency in adults with developmental stuttering

question of whether speakers have a fixed or flexible planning unit for articulation, that is, whether speakers have a lower or upper boundary of advanced planning before articulation is initiated. Different stances on this question propose that speakers start articulation on the basis of a single encoded phoneme (e.g. Dell et al., 1993) or obey a minimum of fully encoded units, ranging from a syllable (e.g. Schriefers \& Teruel, 1999) to the fully encoded phonological word (e.g. Damian \& Dumay, 2007; Levelt \& Wheeldon, 1994). In an object naming study, Griffin (2003) found that speakers incorporate not only the length of the current object's name but also on the length of the following object name in their decision when to initiate articulation: when the second object's name is longer, they start articulation of the first object name later to allow for more planning time to temporally align planning and execution. Meyer et al. (2007) further investigated this 'reversed length effect' testing competing hypothesis as to how speakers manage the advanced planning and execution of multisyllabic utterances. They conclude that speakers can adjust to factors such as word length, utterance format and speech rate for an optimal timing between planning and execution of upcoming utterances. Cholin et al. (2011) in line with Meyer et al. (2003) concluded that speakers are flexible in their decision when to start speaking, that is, on the basis of the first syllable or when all syllables belonging to a multisyllabic phonological word are fully encoded. In either case, the important prerequisite is that syllables belonging to one phonological word, i.e., the respective planning unit, are fully specified during syllabification.

Studies reporting length effects in stuttering attributed these effects to an unstable motor control system/ increased motor complexity that deteriorates with increasing length (e.g. Byrd et al., 2015; Max et al., 2019; Smith et al., 2010) or suffers more due to impaired working memory capacities (Byrd et al., 2012; Sasisekaran \& Weisberg, 2014).

Together, these findings and their interpretations in the separate fields offer a readily explanation for the current results: Not only do we find that errors increase with pseudoword length but, more importantly, our study is the first to report that (pseudoword) length had a 
3. Effects of word length and syllable frequency in adults with developmental stuttering

significant effect on the accuracy of the first syllables. This is in line with the findings of a higher rate in stuttering events in initial syllable, word or utterance position (e.g. Richels et al., 2010; Wingate, 1982, 2002). However, a recent study did not find an effect of utterance position in stuttering rate of AWS, i.e. the first three words of an utterance or phrase were not stuttered more often than other words within a reading passage (Max et al., 2019). Thus, initial syllables in disyllabic pseudowords were significantly more fluently and accurately produced than initial syllables in three- and four-syllabic pseudowords. The speech preparation task with the explicit instruction to start articulation first when speakers feel the urge to speak ensured that speakers were not under pressure as is the case in immediate naming studies in which speakers may opt to use smaller planning chunks to follow instruction a to respond as fast as possible.

Moreover, participants seem to have treated each pseudoword as one planning unit. AWS may have played off the anticipated burden of having to coordinate and integrate an increasing number of syllables into one larger utterance frame already on the first syllable. In other words, the anticipation of upcoming struggle showed on the first syllable (very much in the vein of Bloodstein's (1975) anticipatory struggle hypothesis, for a review see Brocklehurst et al., 2013).

Lastly, we need to address the significant two-way interaction between pseudoword length and syllable frequency. AWS made more errors on high-frequency than on low-frequency initial syllables in shorter, two- and three-syllabic pseudowords but changed this error pattern for longer four-syllabic pseudowords. Here, we can only speculate that the foursyllabic items have triggered other cognitive coping strategies that made the low-frequency syllables in these longer chunks more susceptible to errors.

To sum up, although our results did not show the expected syllable-frequency effects in stutter rates and even an inversed syllable frequency effect in error rates, we still hold onto the dual route account within the Levelt et al. (1999) model as it covers the entire planning 
3. Effects of word length and syllable frequency in adults with developmental stuttering

cycle and includes a very detailed layout of the word-form encoding process. Especially with respect to the hypothesized mental syllabary and the dual route account, it can capture how fluent speakers benefit from stored precompiled motor programs while stuttering individuals lack this advantage and are therefore forced to find compensatory strategies. Moreover, the Levelt et al. (1999) model offers a framework that readily explains the length effects in fluent speakers that can adapt to changing communication settings by balancing the interplay between initiation of articulation and advanced phonological planning. Likewise, this model can explain length effects in speakers with less flexibility and more communication pressures.

An alternative model that offers an explicit account for stuttering is the GODIVA (Gradient Order DIVA) model (Bohland et al., 2010) which is an extension of the DIVA (Directions Into Velocities of Articulators) model (Guenther et al., 2006). In the GODIVA model, stuttering is assumed to occur due to an enhanced dopaminergic level and/or a white matter impairment leading to persistent problems in proceeding from one syllable to the next (Civier et al., 2013). This mechanism is similar to our suggested delayed syllable-release hypothesis from the phonological level to phonetic levels within the Levelt et al. (1999) model. The DIVA/GODIVA does not operate over larger utterances and does not cover syllabification processes in connected speech but does offer a very thorough neurocomputational account for the lower levels of word-form encoding that also includes accounts for a variety of speech impairments (see Kearney \& Guenther, 2019). Bringing these models together might entail a base to integrate findings from fluent and non-fluent speakers.

\section{Conclusion}

The investigation of syllable frequency effects in pseudowords of varying length in AWS has shown that high-frequency syllables were not produced more fluently than low-frequency syllables as was predicted against the background of significant syllable frequency effects in fluent speakers. The finding that AWS also produced more errors on high-frequency 
3. Effects of word length and syllable frequency in adults with developmental stuttering

syllables was interpreted in light of stored motor programs that are more fragile in the stuttering system and that forces AWS to apply compensatory strategies that might involve higher error rates. The investigation of the interplay of syllable frequency and length effects offers important insights into underlying processes in phonetic encoding in AWS and emphasizes the necessity to integrate findings from non-fluent speakers in current models of language and speech production.

\section{Acknowledgements}

This study is part of the PhD dissertation of Alexandra Korzeczek at Georg-August University Göttingen. We are thankful to Meike Drewes, Jacqueline Krebs and Rebekka Weidenmüller for contributing with expert knowledge to the analysis of speech samples. 


\section{Fluent speech: neural basis of sensorimotor plasticity in developmental stuttering}

Authors: Alexandra Korzeczek ${ }^{1, \#}$, Annika Primaßin ${ }^{1,2 \#}$, Alexander Wolff von Gudenberg ${ }^{3}$, Peter Dechent ${ }^{4}$, Walter Paulus ${ }^{1}$, Martin Sommer ${ }^{1}$, and Nicole E. Neef ${ }^{1}$

\section{Affiliation:}

1Department of Clinical Neurophysiology, Georg-August-University, Göttingen, Germany 2Present address: FH Münster University of Applied Sciences, Münster School of Health (MSH), Münster, Germany

${ }^{3}$ Institut der Kasseler Stottertherapie, Bad Emstal, Germany

${ }^{4}$ MR Research in Neurology and Psychiatry, Department of Cognitive Neurology, GeorgAugust-University, Göttingen, Germany

\section{Science Reports, under review}

bioRxiv preprint doi: https://doi.org/10.1101/2020.07.27.219360

\section{Authors contributions:}

\#These authors contributed equally

AK, AP, PD, WP, MS, and NEN conceptualized and designed the study. AP acquired the data. AK and NEN analysed the rs-fMRI data. NEN analysed the dMRI data. AK and NEN interpreted the data, drafted, and revised the manuscript for content. NEN prepared figures. All authors reviewed the manuscript.

The citation style of the publication has been changed to match the citation style of the dissertation. Its reference is included in the general references of the dissertation. 
4. Fluent speech: neural basis of sensorimotor plasticity in developmental stuttering

\section{Abstract}

Developmental stuttering is a fluency disorder with anomalies in the neural speech motor system. Fluent speech requires multifunctional network formations. Currently, it is unclear which functional domain is targeted by speech fluency interventions. Here, we tested the impact of fluency shaping on resting-state fMRI connectivity of the speech planning, articulatory convergence, sensorimotor integration, and inhibitory control network. Furthermore, we examined white matter metrics of major speech tracts. Improved fluency was accompanied by an increased synchronization within the sensorimotor integration network. Specifically, two connections were strengthened, left laryngeal motor cortex and right superior temporal gyrus showed increased connectivity with the left inferior frontal gyrus. The integration of the command-to-execution and auditory-motor pathway was strengthened. Since we investigated task-free brain activity, we assume that our findings are not biased to network activity involved in compensation. No alterations were found within white matter microstructure. But, brain-behavior relationships changed. We found a heightened negative correlation between stuttering severity and fractional anisotropy in the superior longitudinal fasciculus, and a heightened positive correlation between the psychosocial impact of stuttering and fractional anisotropy in the right frontal aslant tract. Taken together, structural and functional connectivity of the sensorimotor integration and inhibitory control network shape speech motor learning. 
4. Fluent speech: neural basis of sensorimotor plasticity in developmental stuttering

\section{Introduction}

Fluent speech requires a complex interplay of multiple neuronal networks. These networks incorporate speech planning (Andreatta et al., 2010; Price, 2012), sensorimotor integration (Behroozmand et al., 2015; Darainy et al., 2018; Hickok et al., 2011; Tourville et al., 2008), articulatory convergence (Brown et al., 2005; Guenther, 2016; Turkeltaub et al., 2002), and the inhibition of competitive processes (Ghahremani et al., 2018; Xue et al., 2008). In developmental stuttering, a heritable speech fluency disorder (Kraft \& Yairi, 2012) of unknown origin (Büchel \& Sommer, 2004; Chang, Garnett et al., 2018), neuroimaging studies indicate aberrant brain activity and connectivity, in particular in brain structures that convey the abovementioned functions (for an overview see e.g. Etchell et al., 2018). However, especially in adults who experienced lifelong stuttering it is difficult to differentiate core neural deficits from stuttering-induced neural signatures, respectively, intervention induced neuroplasticity from compensatory network activity. Thus, our understanding of neurophysiological mechanistic principles of stuttering and its neural remediation remains limited.

Neuroimaging studies provide much of the early evidence implicating particular brain regions in developmental stuttering (Brown et al., 2005). Quantitative meta-analyses link stuttering to reduced left fronto-parietotemporal speech network activity while greater speech fluency of affected individuals is associated with boosted co-activations of homologue right fronto-parietotemporal areas (Belyk et al., 2015, 2017; Budde et al., 2014; Neef et al., 2015). In addition to irregular activity patterns, further imaging findings indicate white matter deficits (Cai, Tourville et al., 2014; Connally et al., 2014; Cykowski et al., 2010; Sommer et al., 2002; Watkins et al., 2008) in the dorsal language pathway (KronfeldDuenias et al., 2016a; Neef et al., 2018), the frontal motor pathway (Kronfeld-Duenias et al., 2016b; Neef et al., 2018) and interhemispheric connections between the sensorimotor cortices (Neef et al., 2015). Movement control engages the basal ganglia and the cerebellum, further crucial brain regions that show abnormal activity and connectivity in 
4. Fluent speech: neural basis of sensorimotor plasticity in developmental stuttering

adults with persistent developmental stuttering (Connally et al., 2014; Giraud et al., 2008; Metzger et al., 2018; Watkins et al., 2008). Taken together, findings give rise to different theories of stuttering, which is seen as a problem of sensorimotor integration and timing, either caused by a basal ganglia dysfunction or caused by a disconnection of cortical speech regions (Civier et al., 2013).

Speech production in stuttering is characterized by sound and syllable repetitions, sound prolongations and speech blocks, which can be accompanied by physical concomitants such as facial grimacing, head or limb movements. One common approach to overcome these characteristic motor signs of stuttering is fluency shaping, a speech restructuring method that require individuals with developmental stuttering to learn a changed speech pattern. In the current study, this learning process was embedded in an intensive two-week on-site intervention and a one-year stand-alone speech training that required computerassisted daily practice (Euler et al., 2009). It is well established that extensive sensorimotor learning induces neuroplasticity (Calmels, 2020). Here it is not the question whether the extensive speech training shapes speech related brain structures and dynamics, but which concrete networks are addressed.

One suitable approach to scrutinizing learning-induced neuroplasticity is the use of restingstate functional magnetic resonance imaging ( $r s-f M R I)$. On the one hand, $r s-f M R I$ is free from confounds of task performance, particularly in participants who may present symptoms such as physical concomitants during speaking. Thus, task-free brain activity assesses changes in brain dynamics that are not biased by differences in how a task is performed in pre-learning versus post-learning condition (Vahdat et al., 2011). On the other hand, it is widely assumed that spontaneous ongoing global activity of the brain at rest is highly structured, closely relates to underlying anatomical connectivity and reflects local neuronal dynamics, signal transmission delay and genuine noise (Deco et al., 2011). It has been shown that even under resting-state condition brain areas show activity changes with 
4. Fluent speech: neural basis of sensorimotor plasticity in developmental stuttering

learning and correlated activity increases between learning related areas (Albert et al., 2009; Darainy et al., 2018; Vahdat et al., 2011).

Until today task-free brain activity has been studied twice to test stuttering intervention induced neuroplasticity (Lu et al., 2012; Lu et al., 2017). In the earlier study, an independent component analysis was used to identify the spatial map of the speech-language network at an individual level. Selected speech-language components were fed into group-wise random effects two sample t-tests resulting in an intervention induced decrease of resting state connectivity in the left declive and vermis area of the cerebellum in the intervention group, but not among the other groups of the study, i.e. fluent controls and individuals who stutter with no intervention. This change in rs-fMRI connectivity was correlated with the intervention-induced change in stuttering frequency (Lu et al., 2012). The analysis approach is uncommon and an attempt to replicate this finding was not successful (Lu et al., 2017). Other studies that tested stuttering intervention induced neuroplasticity used speech tasks such as overt single word reading (Lu et al., 2017), overt sentence reading (Giraud et al., 2008; Kell et al., 2009; Kell et al., 2018; Neumann et al., 2003; Neumann et al., 2005; Neumann, Euler, Bosshardt, Cook, Sandrieser, \& Sommer, 2017), and picture naming (Toyomura et al., 2015). Fluency shaping, the stuttering intervention also tested in the current study, induced a re-lateralization of speech-induced brain activity towards the left hemisphere (Kell et al., 2009; Neumann et al., 2005) possibly due to an improvement of auditory-motor mapping (Kell et al., 2018) and a restructuring of prosody production (Neumann et al., 2018). Across stuttering intervention studies, induced plasticity mainly involved the left inferior frontal gyrus (Kell et al., 2009; Kell et al., 2018; Lu et al., 2017; Neumann et al., 2018). Task-activity however, encompass all components of fluent speech production such as speech planning, articulatory convergence, sensorimotor integration, and the inhibition of non-desirable movements as well as different cognitive strategies to perform the task. 
4. Fluent speech: neural basis of sensorimotor plasticity in developmental stuttering

Here, we use a longitudinal approach to examine stuttering-intervention-induced improvement in speech fluency and related neurofunctional reorganization. We analyzed rs-fMRI data acquired before and 11 months after the computer-assisted speechrestructuring intervention (Euler et al., 2009). We quantify the synchronicity of spontaneous low frequency fluctuations to characterize the connectivity between functionally related brain hubs of speech planning (Neef et al., 2016), articulatory convergence (Guenther, 2016), speech related sensorimotor integration (Darainy et al., 2018), and speech motor inhibitory control (Ghahremani et al., 2018; Neef et al., 2016). We test time-dependent changes in rs-fMRI connectivity (Deco \& Corbetta, 2011) in persons who stutter who participated in the stuttering intervention (PDS+). We control for the specificity by studying two control groups, i.e. patients with developmental stuttering not taking part in any stuttering intervention (PDS-) and healthy controls (HC). Furthermore, we test whether intensive stuttering intervention induces changes in white matter tissue properties within two tracts thought to carry signals critical for speech fluency (Kronfeld-Duenias et al., 2016a, 2016b), the superior longitudinal fasciculus (SLF), the frontal aslant tract (FAT). In addition, as a control we examine a tract crucial for current rs-fMRI findings. Finally, we explore correlations between neuroplasticity and behavior. 
4. Fluent speech: neural basis of sensorimotor plasticity in developmental stuttering

\section{Methods}

Participants

Current data have been collected during a dissertation project (Primaßin, 2019) that evaluated the long-term effects of an intensive stuttering intervention on white matte integrity and task-related brain activity. Seventy-six right-handed, monolingual speakers of German participated on a voluntary basis in the current controlled trial. Exclusion criteria were any speech or language disorder other than developmental stuttering, neurological impairment, drug abuse, or medications that act on the CNS. None of the PDS- took part in any stuttering therapy during the entire study period. For analysis, we excluded the data of three participants who in spite of being informed participated in a different stuttering intervention, of four participants with missing behavioral or rs-fMRI data and of one participant with rs-fMRI motion artifacts. Thus, rs-fMRI data analysis comprised 22 PDS+ (2 females, mean age $25.6 \pm 11.7$ ), 18 PDS- (2 females, mean age $34.8 \pm 7.0$ ), and $28 \mathrm{HC}$ (4 females, mean age $25.1 \pm 7.4$ ). The groups were matched with regard to sex and handedness (Oldfield, 1971). PDS- were older and had a higher education score than participants in the two other groups (see Table 10). Since education correlated with age, $r=0.483, p<.001$, only age was included as a covariate in the statistical analyses. Speech fluency (Stuttering severity index, SSI-4) (Riley et al., 2004) of all participants was assessed prior to each MRI session. Stuttering severity was similar in stuttering groups, HC showed lower SSI-4 scores due to their fluent speech (Table 10 and appendix C, Table 1 and 2). In addition, a self-assessment of the psycho-social impact of stuttering (Overall Assessment of the Speaker's Experience of Stuttering, OASES) (Yaruss et al., 2016) indicated that PDS+ suffered more from stuttering than PDS-, which might possibly relate to age and agerelated requirements of living. 
4. Fluent speech: neural basis of sensorimotor plasticity in developmental stuttering

Table 10. Demographic information of participants.

\begin{tabular}{|c|c|c|c|c|c|}
\hline & PDS+ & PDS- & $\mathrm{HC}$ & $\begin{array}{l}\text { Test- } \\
\text { statistics (df) }\end{array}$ & $\begin{array}{l}\text { two-sided } \\
p \text {-value }\end{array}$ \\
\hline$n$ & 22 & 18 & 28 & & \\
\hline Age, years & $25.6 \pm 11.7$ & $34.8 \pm 7.0^{*}$ & $25.1 \pm 7.4$ & $7.58(2,65)^{i}$ & .001 \\
\hline Sex ratio & $20: 2$ & $16: 2$ & $24: 4$ & $-{ }^{\mathrm{ii}}$ & .89 \\
\hline Education $^{\mathrm{a}}$ & $2(1.0)^{\#}$ & $6(3.0)$ & $3(2.8)$ & $27.49(12) \mathrm{iii}$ & $<.001$ \\
\hline Handedness & $91(12)$ & $91(33)$ & $100(33)$ & $0.04(2,68) \mathrm{iii}$ & .98 \\
\hline SSI-4 at T1 & $25(14.3)^{\#}$ & $14(11.3)$ & - & $2.56^{\mathrm{iv}}$ & .010 \\
\hline SSI-4 at T2 & $9(10.5)$ & $12.5(11.0)$ & - & $-1.31^{\text {iv }}$ & .194 \\
\hline OASES at T1 & $3.0(0.6)^{\#}$ & $2.0(0.4)$ & - & $4.70^{\mathrm{iv}}$ & $<.001$ \\
\hline OASES at T2 & $1.9(0.5)$ & $2.0(0.5)$ & - & $-0.65^{\text {iv }}$ & .516 \\
\hline Onset, years & $4.8 \pm 3.0$ & $5.0 \pm 3.6$ & - & $0.22^{\mathrm{iv}}$ & .839 \\
\hline Interval, months & $11.6 \pm 1.0$ & $11.6 \pm 1.4$ & $11.4 \pm 0.8$ & $0.95(2)^{i i i}$ & .623 \\
\hline
\end{tabular}

Note. Interval/ratio -scaled variables are presented as mean \pm standard deviation. Ordinal-scaled variables are presented as median (interquartile range). ${ }^{*}$ significantly different from both other groups in post hoc comparisons $(p<.001)$, " $s$ ignificantly different from stuttering controls $(p<.001)$, 'oneway independent ANOVA, "Fisher's exact test, iiiKruskal-Wallis test, ivMann-Whitney test, ${ }^{a} 1=$ still attending school, 2 = school, 3 = high school, $4=<2$ years college, $5=2$ years of college, $6=4$ years of college, $7=$ postgraduate

The ethical review board of the University Medical Center Göttingen, Georg August University Göttingen, Germany, approved the study, and all participants provided written informed consent, according to the Declaration of Helsinki, before participation. All participants participated in two MRI sessions (T1 and T2) separated by 10 to 15 months. The scanning interval was similar between groups (Table 1). PDS+ were scanned pre- (T1) and post-intervention (T2). 
4. Fluent speech: neural basis of sensorimotor plasticity in developmental stuttering

Intensive stuttering intervention and follow-up care

PDS+ took part in the Kasseler Stotter Therapie (Euler et al., 2009) that incorporates fluency shaping with computer assisted biofeedback during a two-week on-site and a one-year follow-up treatment. Fluency shaping reconstructs patterns of vocalization, articulation and respiration, resulting in prolonged speech, soft voice onsets of initial phonemes and a smooth transition between sounds. The follow-up period consists of two refresher courses at the therapy center, one month and ten months after the intensive training, respectively, as well as a daily biofeedback-assisted practice of the new speech patterns. The computer monitored daily practice intensity.

\section{Assessment and statistical analysis of behavioral data}

Two experienced speech and language pathologists (one of them was AP) assessed changes in speech fluency with the SSI-4. Each speech sample comprised 488 to 500 syllables. To ensure interrater reliability, both raters analyzed nine randomly chosen participants, three from each group. Reliability estimates, as assessed with Krippendorff's Alpha, ranged between 0.84 and 0.96 , indicating a good interrater reliability. The participants' experience with stuttering was assessed with the German version of the OASES (Yaruss \& Quesal, 2014). We assessed behavioral changes as a change of the total scores of the SSI-4 and OASES between T1 and T2 using R (version 3.5.3). We ran robust mixed ANOVAs on trimmed means with group as between-factor and time as withinfactor using the package WRS (R. Wilcox' robust statistics functions, version 0.37; Wilcox, 2019). Post hoc we applied Wilcoxon signed-rank tests.

\section{Definition of four speech-related semi-discrete brain networks}

Fluent speech production engages large-scale brain networks conveying emotional, linguistic, cognitive, sensory, and motor functions. Among these processes, a dysfunctional speech planning, articulatory convergence, sensorimotor integration or motor inhibition most likely cause the primary motor signs of stuttering, which are sound and syllable 
4. Fluent speech: neural basis of sensorimotor plasticity in developmental stuttering

repetitions, sound prolongations and speech blocks. Here, we distinguished four semidiscrete brain networks consisting of brain regions that are recruited for any of these functions (Figure 12).

Selected brain region of dysfunctional speech planning were derived from an earlier fMRI study of our lab investigating imaginary of speaking compared to humming (Neef et al., 2016) (Figure 12 A). Articulatory convergence seeds originated from a combined ALE metaanalyses (Guenther, 2016) on brain imaging studies of simple articulatory movements of jaw, larynx, lips, tongue, and respiratory system (Figure 12 B). Speech related sensorimotor integration seeds were derived from a listen and repeat localizer task in a brain imaging study of sensorimotor plasticity in speech motor adaptation (Darainy et al., 2018) (Figure 12 C). Motor inhibition seeds that involve common areas of inference resolution, action withholding, and action cancellation were derived from a meta-analysis (Zhang et al., 2017). We added the subthalamic nucleus seed to the inhibition network to account for the dedicated involvement of this structure in response inhibition (Aron \& Poldrack, 2006) (Figure $12 \mathrm{D}$ ). We created spherical seeds with a radius of $6 \mathrm{~mm}$ for all ROls. Coordinates for brain hubs involved in speech related sensorimotor integration can be found in Table 11. Seeds for the remaining three networks are listed in appendix C, Table 3 to 5 . Seed ROls did not overlap. 
Figure 12. The four semi-discrete functional networks for the ROI-to-ROI resting state fMRI analysis.
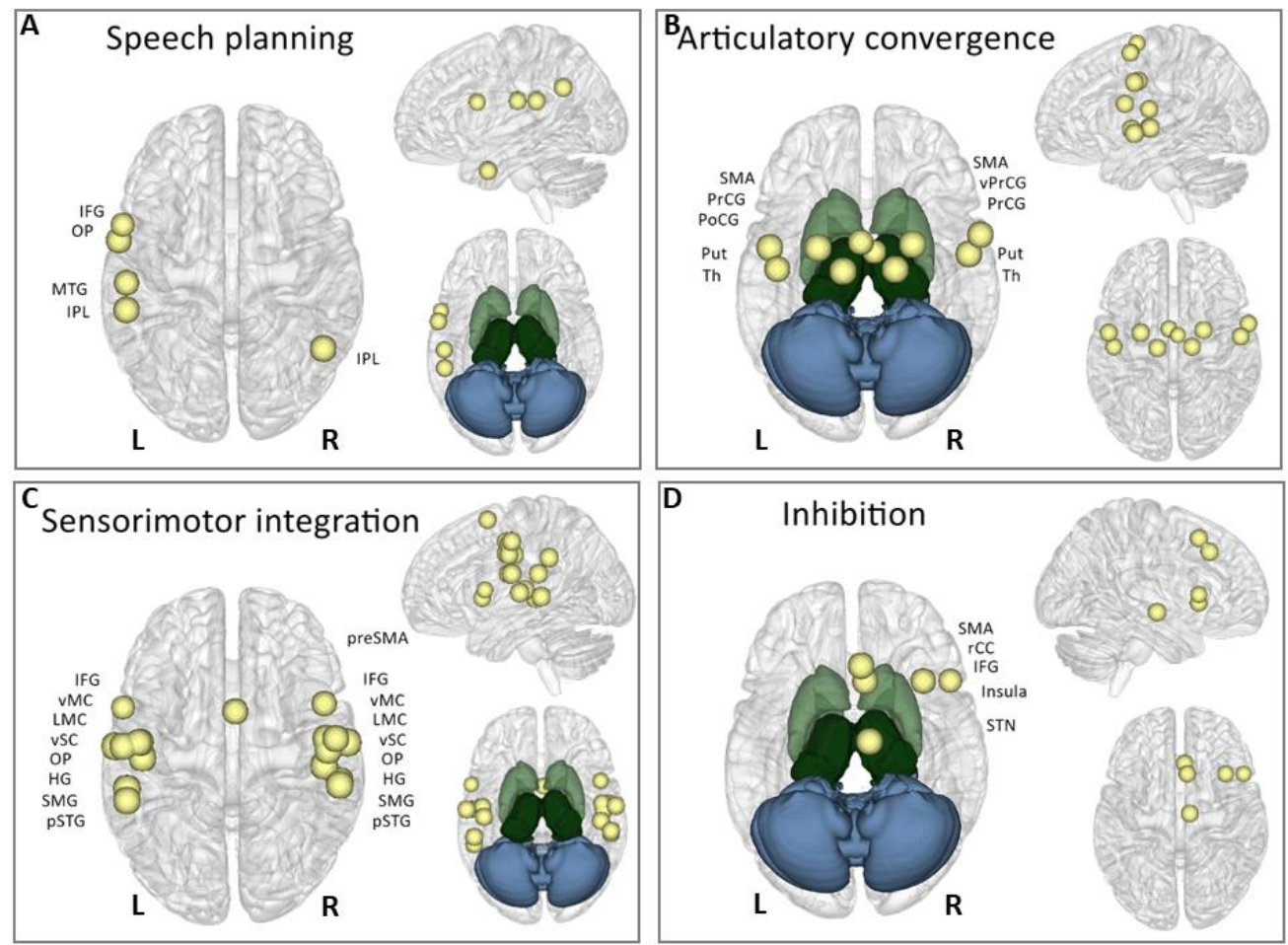

Note. Spheres with a diameter of $6 \mathrm{~mm}$ served as regions of interest and are displayed on rendered surfaces of the $\mathrm{MNI}$ standard brain. Intervention effects were tested for speech planning (A), articulatory convergence (B), sensorimotor integration (C), and motor inhibition (D). HG = Heschl's gyrus, primary auditory cortex; IPL = inferior parietal lobe; IFG = inferior fronatal gyrus, Broca's region; $\mathrm{LMC}=$ laryngeal motor cortex; $\mathrm{MTG}=$ middle temporal gyrus; $\mathrm{OP}=$ parietal operculum; $\mathrm{PoCG}$ $=$ postcentral gyrus; PrCG = precentral gyrus; preSMA = pre-supplementary motor area; PSTG = posterior superior temporal gyrus; Put = putamen; $\mathrm{rCC}=$ rostral cingulate zone; SMA = supplementary motor area; $\mathrm{SMG}=$ supramarginal gyrus; $\mathrm{STN}=$ subthalamic nucleus; $\mathrm{Th}$ = thalamus; $\mathrm{vMC}=$ ventral primary motor cortex; $\mathrm{vPrCG}=$ ventral precentral gyrus; $\mathrm{vSC}=$ ventral primary somatosensory cortex. 
4. Fluent speech: neural basis of sensorimotor plasticity in developmental stuttering

Table 11. Brain hubs of speech-related sensorimotor integration.

\begin{tabular}{lllll}
\hline Brain hub - anatomical label & ROI Label & X & Y & Z \\
\hline Inferior frontal gyrus, posterior- & L IFG & -56 & 8 & 8 \\
ventral, Broca's region & R IFG & 48 & 10 & 2 \\
& R preSMA & 2 & 6 & 60 \\
Pre-supplementary motor area & L vMC & -48 & -10 & 42 \\
Ventral primary motor cortex & R vMC & 54 & -8 & 4 \\
& L LMC & -47 & -10 & 34 \\
Laryngeal motor cortex & R LMC & 49 & -8 & 35
\end{tabular}

Ventral primary somatosensory

cortex

$\begin{array}{llll}\text { L vSC } & -56 & -12 & 44\end{array}$

$\begin{array}{llll}R \text { vSC } & 50 & -14 & 34\end{array}$

Parietal Operculum, secondary

somatosensory cortex

$\begin{array}{llll}\text { L OP } & -60 & -12 & 20\end{array}$

$\begin{array}{lllll} & \text { R OP } & 60 & -10 & 20 \\ \text { Supramarginal gyrus } & \text { L SMG } & -54 & -40 & 32 \\ & \text { R SMG } & 56 & -32 & 2\end{array}$

Heschl's gyrus, primary auditory

cortex

$\begin{array}{llll}\text { L HG } & -46 & -18 & 6\end{array}$

$\begin{array}{lllll} & \text { R HG } & 48 & -22 & 8 \\ \text { Posterior superior temporal gyrus } & \text { L pSTG } & -54 & -34 & 3 \\ & \text { R pSTG } & 56 & -30 & 3\end{array}$

Note. All coordinates refer to MNI-space. $\mathrm{L}=$ left, $\mathrm{R}=$ right. Coordinates were derived from a listen and repeat speech task (Darainy et al., 2018). 
4. Fluent speech: neural basis of sensorimotor plasticity in developmental stuttering

\section{MRI acquisition protocol}

MRI data were acquired in a 3 Tesla Siemens Magnetom Tim Trio scanner (Erlangen, Germany) using an eight-channel phased-array head coil at the University Medical Center Göttingen, Germany. Sagittal T1 weighted structural data were acquired with a 3D turbo fast low angle shot $(\mathrm{FLASH})$ sequence $(\mathrm{TR}=2250 \mathrm{~ms}, \mathrm{TE}=3.26 \mathrm{~ms}, \mathrm{TI}=900 \mathrm{~ms}$, flip angle $=9^{\circ}, 256 \mathrm{~mm}$ FoV, $7 / 8$ Fourier phase encoding) as whole-brain anatomical reference data at a spatial resolution of $1 \cdot 1 \cdot 1 \mathrm{~mm}^{3}$ voxel size $(256 \cdot 256$ matrix $)$. For resting-state fMRI a gradient-echo echo planar imaging $(\mathrm{EPI})$ sequence $(\mathrm{TR}=1800 \mathrm{~ms}, \mathrm{TE}=30 \mathrm{~ms}$, flip angle $=$ $70^{\circ}$, parallel acquisition factor 2, $192 \mathrm{~mm}$ FoV, 33 slices, 194 volumes) was used with isotropic voxels at $3(\mathrm{~mm})^{3}$ and a $64 \times 64$ acquisition matrix. We acquired two six-minute rsfMRI time series at T1 and at T2, respectively, while participants fixated a cross in an open eyes condition. Due to different head sizes the rs-fMRI data did not fully cover the cerebellum in some participants. Therefore, the cerebellum was excluded in further rs-fMRI analyses. Diffusion-weighted MRI (dMRI) was performed using a spin-echo EPI sequence ( $\mathrm{TR}=10100 \mathrm{~ms}, \mathrm{TE}=93 \mathrm{~ms}$, parallel acquisition factor 2, 6/8 Fourier phase encoding, 243mm FoV, acquisition matrix: $128 \cdot 128,74$ slices, voxel size $1.9 \times 1.9 \times 1.9 \mathrm{~mm}^{3}$ ) acquiring 64 image volumes with diffusion weighting along 64 diffusion directions $(b=1000$ $\mathrm{s} / \mathrm{mm}^{2}$ ) and one reference image without diffusion weighting. Participants lay in supine position in the scanner and wore headphones for noise protection, and MRI compatible LCD goggles (VisuaStim XGA, Resonance Technology Inc., Northridge, CA, USA).

\section{Rs-fMRI data processing and analysis}

Structural and functional MRI data were preprocessed and analyzed with CONN functional connectivity toolbox version 18b (Whitfield-Gabrieli \& Nieto-Castanon, 2012). The toolbox is based on Matlab and Statistical Parametric Mapping (SPM). The standard preprocessing pipeline of CONN was used: Structural data were centered to $(0,0,0)$ coordinates, segmented and normalized to $\mathrm{MNI}$-space. Functional data were realigned and centered to 
4. Fluent speech: neural basis of sensorimotor plasticity in developmental stuttering

$(0,0,0)$ coordinates, slice-timing corrected, checked for outliers using $95^{\text {th }}$ percentiles settings (Goto et al., 2016; Power et al., 2012), direct segmented and normalized to MNIspace. Finally, functional images were smoothed with a Gaussian kernel of 8mm FWHM (full-width half-maximum). After these preprocessing steps, functional data were corrected for white matter and cerebro-spinal fluid signals, motion and scrubbing as well as effects of measurements using linear regression. Following regression, a $0.009-0.08 \mathrm{HZ}$ bandpass filter was applied.

To test for an intervention-induced neuroplasticity, we calculated Fisher-transformed correlation coefficients off bivariate $\mathrm{ROI}$-to- $\mathrm{ROI}$ correlations with hemodynamic response function weighting for each of the four predefined semi-discrete speech-related networks (Figure 12). Second-level analyses comprised four global $(3 \times 2 \times \mathrm{ROI})$ mixed model ANCOVAs with Group as between-subjects factor, Time and ROls as within-subjects factors and age with grand mean centering as covariate. Multiple comparison correction was performed with a connection threshold at seed level p-FDR $<.05$ (two-sided) and a seed-level permutation analysis threshold based on a Network Based Statistics by intensity approach (Whitfield-Gabrieli \& Nieto-Castanon, 2012) with p-FDR $<.05$. In case of a significant Group x Time interaction, we run subsequent post hoc analyses to distinguish group effects in those connectivity changes. Effect sizes are reported with beta values, which represent average functional connectivity (Fisher-transformed correlation coefficients) among the tested contrasts.

\section{Diffusion-weighted imaging data processing and analyses}

Diffusion data were preprocessed with tools from the FMRIB Software Library, (FSL 6.0 on a Linux machine with Ubuntu LTS 18.04, http://www.fmrbi.ox.ac.uk/fs//). Fiber tracking was carried out on aligned, distortion corrected, concatenated datasets that were collected across $\mathrm{T} 1$ and $\mathrm{T} 2$ for each subject to ensure that estimates of diffusivity and diffusion anisotropy across sessions were mapped to the same anatomical location for each subject. White matter tracts were identified with the Automated Fiber Quantification software 
4. Fluent speech: neural basis of sensorimotor plasticity in developmental stuttering

implemented in python (pyAFQ, https://github.com/yeatmanlab/pyAFQ) (Yeatman et al., 2012). Within pyAFQ individual b0 images were aligned with the native anatomy via an affine registration and warped to the FSL_HCP1065_FA_1 $\mathrm{mm}$ template via a nonlinear registration. Inverted warp maps were used to register waypoint masks and exclusion masks with individual b0 images. A whole brain tractogram was generated in the native space of each participant with a deterministic tracking algorithm with a fourth-order RungeKutta path integration method ( $1 \mathrm{~mm}$ fixed step size, 8 seed points per voxel, FA threshold 0.2 and $30^{\circ}$, minimum streamline length set to $10 \mathrm{~mm}$ ). We segmented the superior longitudinal fasciculus (SLF), the frontal aslant tract (FAT) and the tract connecting LMC and IFG in the left and right hemisphere, respectively. For tract segmentation the whole brain tractogram was filtered, first with a tract-specific probability map, second with waypoint and exclusion masks and third with cortical endpoint masks. A detailed description is provided in appendix C, Supplementary Material. Visual inspection of the tracts and manual cleaning was performed via TrackVis 0.6 .1 (http://trackvis.org/). Not all tracks could be segmented in all participants as reported in appendix C, Table 8 to 10.

Finally, we fitted the diffusion kurtosis model, implemented in DIPY (https://dipy.org/) (Garyfallidis et al., 2014), to the diffusion data separately for each session and projected the fractional anisotropy (FA), mean diffusivity (MD), radial diffusivity (RD) and axial diffusivity (AD) onto the segmented fiber tracts generated by pyAFQ. Therefore, selected tracts were sampled into 100 evenly spaced nodes, spanning the two termination points. To determine changes in diffusion properties we built the difference wave between T2 and T1 and averaged the values of the inner 60 nodes. This procedure was adopted from a report of rapid white matter changes after an intensive reading intervention (Huber et al., 2018). The change in diffusivity and diffusion anisotropy was tested against zero separately for each tract and each group and Bonferroni-corrected to account for multiple testing. 
4. Fluent speech: neural basis of sensorimotor plasticity in developmental stuttering

\section{Correlation analyses}

Finally, Pearson correlations were calculated in MATLAB (R2018b) to test brain behavior relationships for rs-fMRI and dMRI metrics.

Data availability statement

The data that support the findings of this study are available from the corresponding author upon reasonable request.

\section{Results}

Computer-assisted intensive intervention improved speech fluency and well-being

A reduction in the total scores of the Stuttering Severity Index (SSI-4) and the Overall Assessment of the Speaker's Experience of Stuttering (OAESES) as seen here indicates a positive outcome of stuttering intervention. The robust ANOVA for stuttering severity revealed a significant interaction of Group by Time, $Q=24.44, p<.001$, an effect of Group $Q=48.38, p<.001$, and an effect of Time $Q=50.99, p<.001$. Post hoc tests revealed that in the intervention group, stuttering severity decreased from $T 1$ to $T 2, V=253, p<.001, r$ $=-0.87$ (Figure 13A). In contrast, in the non-intervention groups the SSI-4 scores remained unchanged with $V=63, p=.81, r=-0.06$ for PDS- and $V=58.5, p=.38, r=-0.17$ for HC. Similarly, the robust ANOVA for the speaker's experience of stuttering revealed a significant interaction of Group by Time, $Q=66.73, p<.001$, an effect of Group $Q=20.39, p<.001$ and an effect of time $Q=74.17, p<.001$. Post hoc tests revealed that a decrease in the OASES-scores between T1 and T2 emerged only in PDS+, V = 253, $p<.001, r=-0.87$ (Figure 13B). PDS- yielded no changes in their experience with stuttering, $V=110.5$, $p=.29, r=-0.25$. Behavioral outcome measures are summarized in appendix $C$, Table 6. 
4. Fluent speech: neural basis of sensorimotor plasticity in developmental stuttering

Intervention strengthened a dedicated speech motor learning connection

Only one of the four global mixed model ANCOVAs revealed significant results. When seeds of the sensorimotor integration network were inserted in the analysis, the left IFG showed a Group x Time x Target interaction with $X(28)=48.57$, Intensity $=21.57, p=.005$. Specifically, the factors Group and Time had an effect on the connectivity between left IFG and left $\mathrm{LMC}$, beta $=0.12, F(2,64)=8.71, p=.007$, the left IFG and the left SMG, beta $=0.24, F(2,64)=7.4, p=.01$, and the left IFG and the right $p S T G$, beta $=0.13$, $F(2,64)=5.47, p=.034$ (Figure 13A, upper panel). There were no main effects of Group or Time. Results of connection-wise, post hoc two-sample t-tests controlling for age are listed in Table 12. For the left IFG-to-left LMC connection the analyses revealed a higher change in connectivity for PDS+ when compared to $\mathrm{HC}$ and when compared to PDS-. For the left IFG-to-right pSTG connection a higher change in connectivity was evident in PDS+ compared to HC and showed a trend when inserting PDS+ and PDS-. For the left IFG-toSMG connection the analyses revealed a higher change in connectivity for PDS- compared to $\mathrm{HC}$ and showed a trend for PDS+ compared to $\mathrm{HC}$ (appendix C, Figure 2). A final posthoc $2 \times 17$ ANCOVA with an analysis-level correction for multiple comparison of seeds and targets confirmed the influence of Time on the synchronicity between left IFG and left LMC in PDS+ with beta $=0.1, \mathrm{t}(20)=5.47, \mathrm{p}=.003$ (Figure 13A, lower panel). 
Figure 13. Intensive computer-assisted intervention reduced stuttering and strengthened IFG-to-LMC rs-connectivity within the sensorimotor integration network.
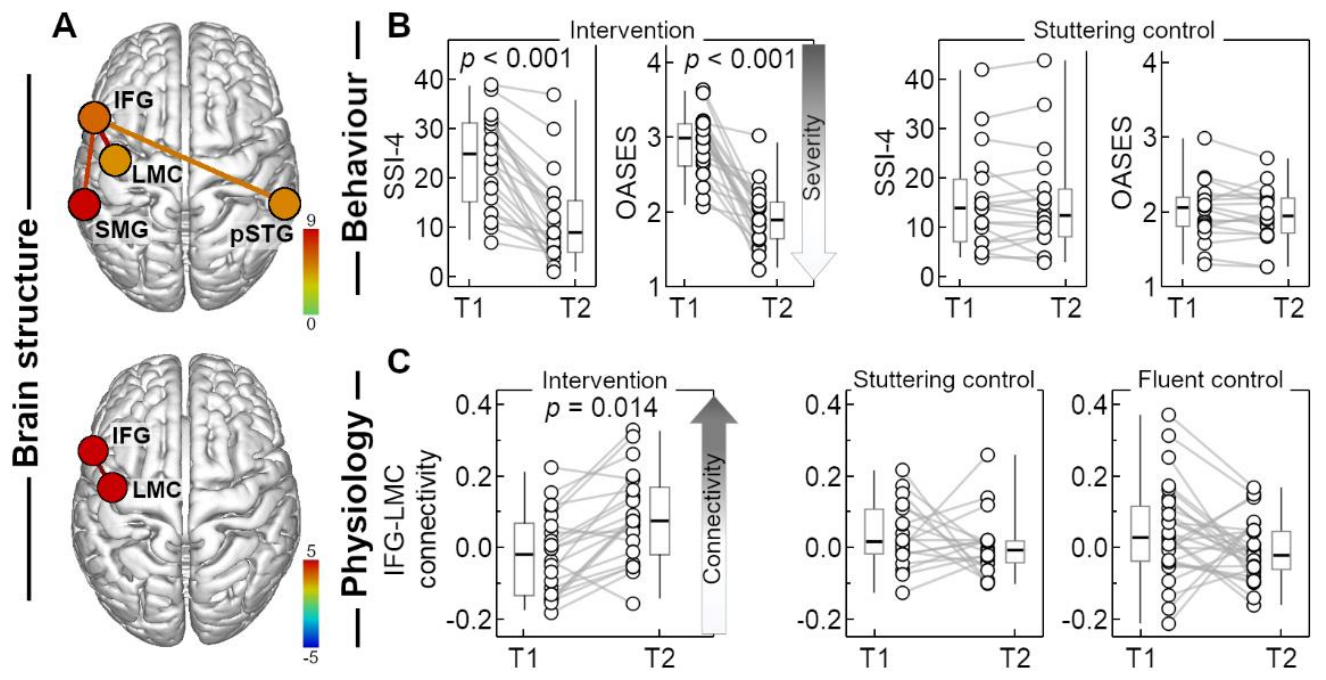

Note. On the behavioral level (upper panel) stuttering severity decreased between pre- (T1) and 11months post-intervention (T2) as shown by the reduced SSI-4 scores, scaling from 0 (none) to 46 (very severe), and the reduced OASES-scores, scaling from 1 (mild) to 5 (severe) in the intervention group (PDS+). In contrast, stuttering controls showed no changes of stuttering severity. On the physiological level (lower panel) ROI-to-ROI functional connectivity analysis revealed a boosted correlation within the speech-related sensorimotor integration network in the intervention group. Left IFG-LMC-correlation was increased as shown by comparison of beta estimates between pre- (T1) and one-year post-intervention (T2). The two control groups, stuttering control and fluent controls, showed no changes of connection strength. IFG = inferior frontal gyrus; LMC = laryngeal motor cortex; $\mathrm{pSTG}$ = posterior superior temporal gyrus; SMG = supramarginal gyrus. (D) Illustration of IFG and LMC locations on the rendered surface of the human brain. The result is significant at $p<.05$, analysis-level false discovery rate (FDR) corrected. IFG = inferior frontal gyrus pars opercularis; LMC = laryngeal motor cortex; OASES = Overall Assessment of the Speaker's Experience of Stuttering; $\mathrm{ROI}=$ region of interest; $\mathrm{SSI}=$ Stuttering Severity Index . 
4. Fluent speech: neural basis of sensorimotor plasticity in developmental stuttering

Table 12. Post hoc analyses.

\begin{tabular}{lllll}
\hline $\begin{array}{llll}\text { Seed - target } \\
\text { region }\end{array}$ & Analysis & Beta & $\begin{array}{l}\text { Test } \\
\text { value(DF) }\end{array}$ & $\boldsymbol{p}$ \\
\hline left IFG - LMC & PDS+ vs. HC & 0.14 & $3.77(46)$ & $<.001$ \\
& PDS+ vs. PDS- & 0.12 & $2.47(36)$ & .018 \\
& PDS- vs. HC & 0.024 & $0.41(42)$ & .683 \\
left IFG - SMG & PDS+ vs. HC & 0.08 & $1.8(46)$ & .079 \\
& PDS+ vs. PDS- & -0.05 & $-0.81(36)$ & .426 \\
& PDS- vs. HC & 0.13 & $2.1(42)$ & .042 \\
left IFG - right pSTG & PDS+ vs. HC & 0.14 & $2.89(46)$ & .006 \\
& PDS+ vs. PDS- & 0.11 & $1.85(36)$ & .073 \\
& PDS- vs. HC & 0.035 & $0.51(42)$ & .609 \\
\hline
\end{tabular}

No correlation between rs-connectivity and improvement after stuttering intervention

Neither the change in speech fluency (SSI-4) nor the experience of stuttering (OASES) correlated with rs-connectivity, all $p>.05$.

No changes in diffusion properties due to intervention

None of the three white matter tracts showed a significant change in any of the diffusion properties, FA, MD, RD or AD in PDS+. PWS- showed a change of FA in the left IFG-toLMC tract $(\mathrm{t}(18)=-2.86, \mathrm{p}=.048)$ and $\mathrm{HC}$ showed a change of FA in the left SLF $(t(26)=-4.33, p=.001)$ together with a change of $R D(t(26)=2.71, p=.048)$, and a change of $F A$ in the right SLF $(t(24)=-3.08, p=.021)$. HC showed in addition a change of AD in the right IFG-to-LMC tract $(\mathrm{t}(26)=-3.33, \mathrm{p}=.012)$. Figure 14 illustrates the segmented tracts of the left hemisphere and its FA profiles together with the magnitude of change in FA and MD. Tract specific group averages, standard deviations, t-values and $p$-values for all diffusion metrics are listed in the supplement (appendix C, Table 8 to 10). 
Figure 14. No intervention-related white matter changes in three speech motor tracts.
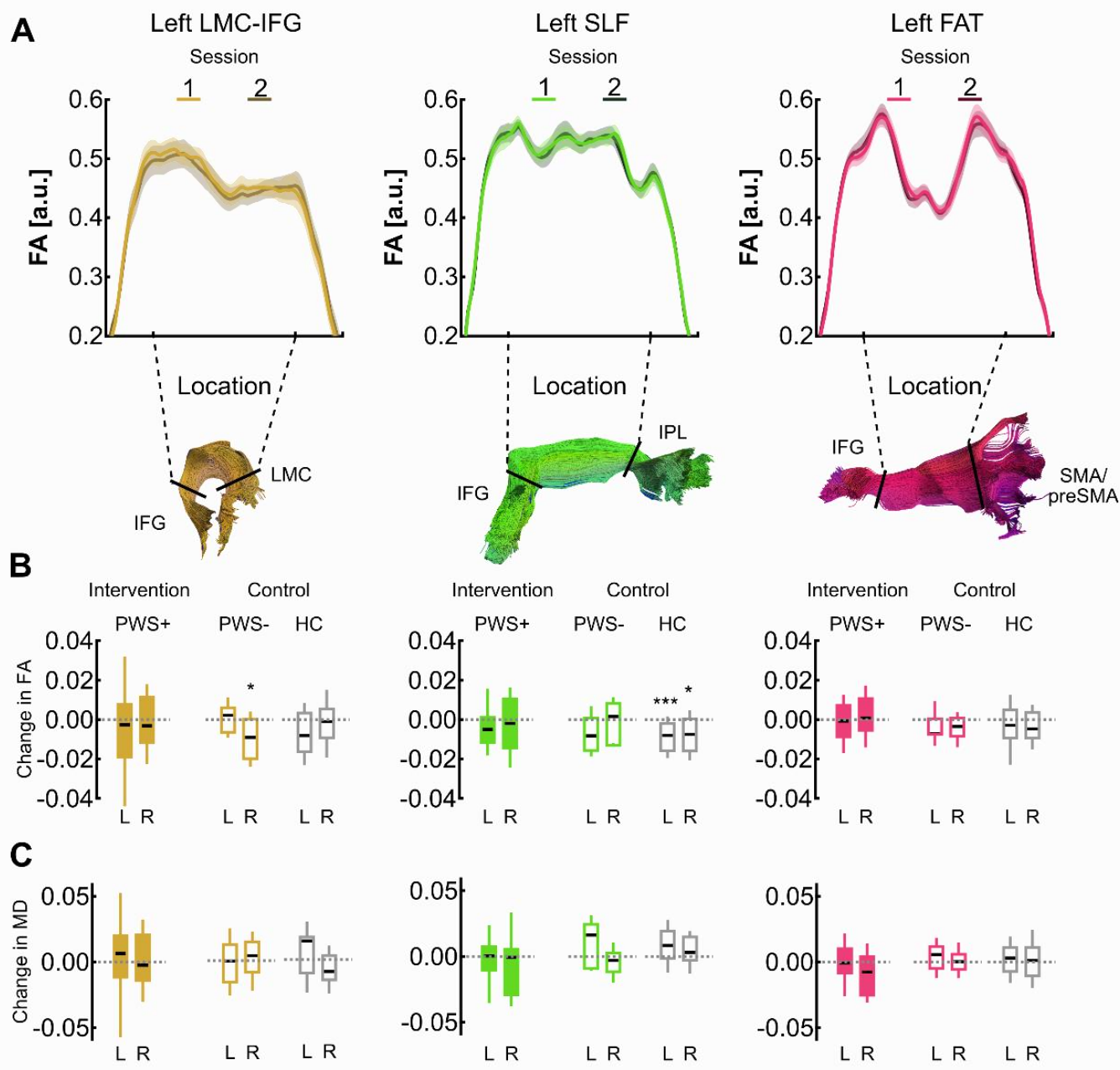

Note. (A) Fractional anisotropy, FA, was mapped onto 100 evenly spaced points connecting cortical speech regions. Each curve represents the group average FA across participants of the intervention group (PWS+, $\mathrm{n}=22$ ) for the white matter connecting the left laryngeal motor cortex $(\mathrm{LMC})$ and the left inferior frontal gyrus (IFG, brown), the left superior longitudinal fasciculus (SLF, green), and the left frontal aslant tract (FAT, red). Pale colors show pre-intervention FA (Session 1) and dark colors show FA after $\sim 11$ months of intervention (Session 2). Shaded areas give \pm 1 standard error of the mean. Each tract was clipped prior to analysis as shown with black boundary lines in corresponding tract renderings. To avoid pruning effects that occur at endpoints of the tract, only the middle $60 \%$ of each tract was considered in the analyses of $F A(B)$ and mean diffusivity, MD (C). Box plots illustrate the magnitude of change observed relative to Session 1 for the left $(L)$ and right $(R)$ hemisphere for the intervention group (filled boxes) and the two control groups (stuttering without intervention, PWS; and fluent speakers without intervention, $\mathrm{HC}$; unfilled boxes). Whiskers indicate \pm one standard deviation. Asterisks indicate a significant increase in FA (B) or decrease in MD (C) at a Bonferronicorrected ${ }^{*} p<.05$ and ${ }^{* * *} p<.001$. 
4. Fluent speech: neural basis of sensorimotor plasticity in developmental stuttering

Relieve from stuttering relates to white matter integrity of SLF and FAT

We calculated point-wise Pearson correlations between diffusion metrics projected to the SLF, FAT and LMC-to-IFG connection and the motor signs of stuttering measured with SSI4. At T2, SSI-4 scores of PWS+ correlated negatively with FA values projected to the posterior part of the left SLF. Bootstrapping with a boot size of 100000 revealed significant point-wise correlations at $p<.005$ with a cluster-size significance at $p<.01$ (Figure 15A, B). Thus, after intervention, mild stuttering was related to higher FA values, while severe stuttering was related to lower FA values in the left SLF. At T1, correlations in the same SLF region did not survive correction for multiple comparisons (Figure 15A, C). We calculated a z-score of the difference between the correlation coefficients. With a difference score of $z=1.79$ and a two-tailed $p=.073$, the correlations between FA at T2 and SSI-4 trended to be higher than the correlation between FA at T1 and SSI-4. No other correlation between SSI-4 and white matter characteristics reached statistical significance.

The psycho-social impact of stuttering, as measured with the OASES, was positively correlated with the middle portion of the right FAT (Figure 15B, D). Thus, after intervention mild suffering from stuttering was related to lower FA values, while severe suffering from stuttering was related to higher FA values in the right FAT. Bootstrapping with a boot size of 100000 revealed significant pointwise correlations at $p<.005$ with a cluster-size significance at $p<.02$. With a difference score of $z=-2.89$ and a two-tailed $p=.004$, the correlation between FA at T2 and OASES was significantly higher than the correlation between FA at T1 and OASES. No other correlation between OASES and white matter characteristics reached statistical significance. 
Figure 15. Brain behavior relationship changed with intervention.
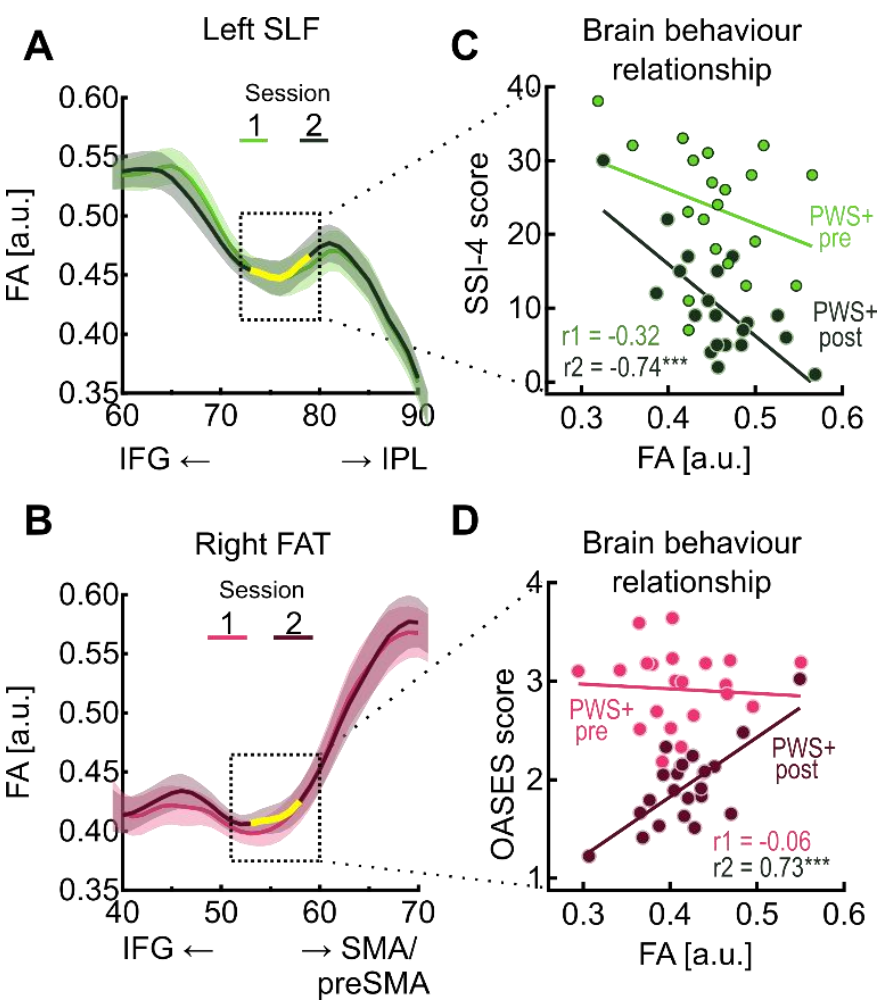

Note. Each curve represents the group average FA across participants of the intervention group (PWS+) from the posterior part of the left superior longitudinal fasciculus (SLF, A), and from the medium part of the right frontal aslant tract (FAT, B). Pale colors show pre-intervention FA (Session 1) and dark colors show post intervention FA (Session 2). Shaded areas give \pm 1 standard error of the mean. (C) Stuttering severity (SSI-4 scores) is plotted against FA values averaged across tract points 73 to 81 framed with a dotted square in A. The Pearson correlation was significant at ${ }_{* * *} p<.001$. Bootstrapping with a boot size of 100000 revealed significant point-wise correlations at $p<.005$ with a cluster-size significance at $p<.01$ highlighted with yellow in $A$. (D) Experience of stuttering (OASES scores) is plotted against FA values averaged across tract points 52 to 61 framed with a dotted square in B. The Pearson correlation was significant at ${ }^{* * *} p<.001$.

Bootstrapping with a boot size of 100000 revealed significant point-wise correlations at $p<.005$ with a cluster-size significance at $\mathrm{p}<.02$ highlighted with yellow in $\mathrm{B}$.

\section{Discussion}

A one-year technology-based speech-restructuring training sustainably facilitated speech fluency of patients with developmental stuttering. As a neural correlate we found a strengthened synchronization of the posterior-ventral IFG pars opercularis with the laryngeal motor cortex within the sensorimotor integration network as evidenced by restingstate functional brain activity. Hence, here we show for the first time that a computer- 
4. Fluent speech: neural basis of sensorimotor plasticity in developmental stuttering

assisted biofeedback-based, intensive speech-training program induced functionally specific, focal and long-lasting changes in task-free brain activity.

\section{Speech-restructuring training changes connectivity within sensorimotor integration network}

Reorganization of brain functions after short-term stuttering interventions have been reported for task-related (Lu et al., 2017; Toyomura et al., 2015) as well as spontaneous MRI signal fluctuations (Lu et al., 2017; Lu, Chen et al., 2010) in fMRI. Strikingly, such studies have reported increased activity of the left IFG, the basal ganglia or a decreased activity of the right hemisphere (Kell et al., 2009; Lu et al., 2017; Neumann et al., 2003; Neumann et al., 2005; Toyomura et al., 2015). These structures however are part of different speech networks. For example, the left IFG attributes to speech motor planning as well as sensorimotor integration (Guenther et al., 2006) whereas right hemispheric activity including the IFG could be part of the inhibition network (Neef et al., 2016) (Figure 12). We thus hypothesized that the intervention under study here could lead to an increased connectivity within the networks of speech motor planning, articulatory convergence or sensorimotor integration network or to a decreased connectivity within the motor inhibition network. Interestingly, connectivity increase was restricted to key functional brain hubs of sensorimotor integration and speech motor learning. Specifically, the observation of an increased rs-fMRI connectivity of the left IFG with the left LMC is new. While previous studies performed hypothesis-free whole-brain analyses, our ROI-to-ROI analysis was hypotheses-driven, and restricted to four semi-discrete networks that closely relate to the stuttering trait and to the behavioral changes that are targeted by the specific speech restructuring intervention. Other speech-related networks such as the network of speech planning, articulatory convergence or motor inhibition remained unchanged. Thus, the intervention under study strengthened the interplay of the neurobiological substrate of sensorimotor integration required to learn a new speech technique. 
4. Fluent speech: neural basis of sensorimotor plasticity in developmental stuttering

Recovered fluent speech relates to a strengthened synchronization of the left IFG with the left laryngeal motor cortex and the right posterior superior temporal gyrus

The studied intervention encompassed one year of learning and practicing a new speech technique. This speech technique comprises soft voice onsets, consonant lenitions, and controlled sound prolongations (Euler et al., 2009). Thus, voicing and timing are the key features under change over the course of the acquisition of the new speech technique. The control of voicing is based on the neural control of the larynx and involves the LMC (Brown et al., 2008; Kristofer E. Bouchard et al.; Olthoff et al., 2008; Rödel et al., 2004; Simonyan et al., 2009; Simonyan \& Horwitz, 2011), while the control of speech timing involves activity of the posterior part of the inferior frontal gyrus pars opercularis (Clos et al., 2013; Long et al., 2016; Neef et al., 2016). Accordingly, the intensive training incorporated two brain regions, the left LMC and the left IFG, that provide essential neural contributions to fluent speech production.

In the current study, we found that the intervention strengthened connectivity between the left IFG and the left LMC. This finding is also consistent with the involvement of the left posterior IFG and left motor cortex in motor learning in general (Papitto et al., 2020), and with the particular involvement of the posterior IFG and the left LMC in speech motor learning (Darainy et al., 2018; Rauschecker et al., 2008). The IFG and the orofacial motor cortex share direct connections (Greenlee et al., 2004), and are commonly co-active under task- and resting-state conditions (Simonyan et al., 2009; Simonyan \& Fuertinger, 2015). Theories on speech motor control assume the posterior region of the IFG to link the target speech unit to an articulatory code that is subsequently implemented by the motor cortices that finally orchestrate the articulators, including the larynx (Guenther, 2016). Hence, vital connectivity between the left IFG and left LMC is particularly important for the acquisition of speech or of a new speech technique.

Intervention also strengthened co-activity between the left IFG and the right pSTG. The speech motor system is required to monitor the auditory feedback signal, to rapidly correct 
4. Fluent speech: neural basis of sensorimotor plasticity in developmental stuttering

small articulatory errors in natural speech. Speech-related auditory feedback control involves the right pSTG and task-related co-activations of the left posterior IFG with bilateral pSTGs (Behroozmand et al., 2015; Guenther et al., 2006; Niziolek \& Guenther, 2013; Tourville et al., 2008). The standard model of speech motor control suggests that the posterior IFG provides feedforward control signals and the PSTG conveys feedback-based corrective signals9. Learning and practicing a new speech technique addresses neural circuitries of auditory feedback monitoring because patients are constantly required to adjust the sound of their speech to fit it to the new auditory target space. We suggest that the increased co-activity between left IFG and right pSTG could reflect the frequent recruitment of both brain regions and auditory feedback control mechanisms during learning and practicing the new speech technique.

Here, we measured MRI signal fluctuations in the absence of response demands or external stimulation to describe intervention-induced changes to the speech function-related sensorimotor integration network. It is assumed that spontaneous brain activity at rest relates to the underlying anatomical circuitry (Deco et al., 2013) as supported by diffusion weighted imaging (Hagmann et al., 2008; Honey et al., 2009). Specifically, it has been suggested that spatially and temporally correlated brain activity at rest arises from neuronal noise between brain areas that share anatomical connections (Deco et al., 2013). In this respect, the current study extends previous studies where task-related changes in brain activity were observed as a result of the very same intensive stuttering intervention (Kell et al., 2009; Kell et al., 2018; Neumann et al., 2003; Neumann et al., 2005). However, taskrelated neuroimaging results from learning studies might be confounded by behavioral changes. Using resting-state activity as a neural marker of neuroplasticity rules out that changes in brain activity were induced by changes in task performance (Darainy et al., 2018). In fact, here we provide a purely neurophysiological index of neuroplasticity in the context of an intensive stuttering intervention. 
4. Fluent speech: neural basis of sensorimotor plasticity in developmental stuttering

In this study, rs-connectivity and behavioral changes did not correlate. Two other long-term intervention studies did not report any correlations between brain activity and behavioral change (Neumann et al., 2003; Toyomura et al., 2015). Correlations of brain activity with enhanced speech fluency in other stuttering intervention studies are based on direct measurements after intervention (Kell et al., 2009; Lu et al., 2012, 2012; Lu et al., 2017), task-based brain activity (Kell et al., 2009; Lu et al., 2017) or on cerebellar rs-connectivity (Lu et al., 2012). The lack of seed regions in the cerebellum is one limitation of the current study. Previous studies reported the cerebellum to be a region of intervention-induced neural reorganization in stuttering (Kell et al., 2009; Lu et al., 2012; Toyomura et al., 2015), a finding that has not been replicated in a more recent study (Lu et al., 2017). Because in our study the field of view of the EPI sequence did not cover the whole brain, making it impossible to include ROls of the cerebellum. In addition, correlational effects between connectivity strength and adherence to practice cannot be investigated as commitment to practice was not documented. Last, due to a limited acquisition time of two years, groups varied significantly in age. Age differences seem to have influenced reported changes in connectivity between left IFG and left SMG (please refer to appendix C, the supplementary discussion).

\section{No long-term changes of diffusion properties in major speech tracts}

Here, long-term plasticity was evident in an increased synchronization of crucial left cortical speech motor regions. Related white matter tracks showed no plasticity in concert with fluency enhancement. Although, this is surprising, since for other domains such as reading intervention white matter changes have been reported (Huber et al., 2018), our findings agree with the only previous DTI investigation (Kell et al., 2009) on stuttering intervention. Still, brain-behavior relationships changed with changes in behavior. After therapy, stuttering severity was negatively correlated with FA in the left SLF such that the higher the FA values in the SLF the milder the stuttering after intervention. One possible interpretation could be that the strength of the SLF facilitates therapy outcome. Furthermore, after 
4. Fluent speech: neural basis of sensorimotor plasticity in developmental stuttering

therapy, FA in the right FAT correlated positively with the psycho-social impact of stuttering, meaning the weaker the suffering from stuttering the lower the FA values in the right FAT. Strikingly, the right FAT is associated with action inhibition and it was recently hypothesized that hyperactive inhibitory circuits contribute to the occurrence of stuttered speech (Neef et al., 2018). The current observation further suggests, that relieve from stuttering is associated with weaker connectivity of related inhibitory structures.

Perspectives with regard to other therapeutic approaches to ameliorate stuttering

Common stuttering interventions consist of (1) speech motor interventions partly modifying or fully reshaping laryngeal, articulatory or respiratory movements, (2) feedback and technology interventions, which, e.g., use delayed auditory feedback to enhance fluency, or visual feedback to support speech motor interventions, (3) behavioral modification interventions, or (4) cognitive interventions improving psychological well-being, selfconfidence, and self-conception. The current and previous studies tested neurofunctional correlates of brain reorganization for the first two approaches. The neurobiological foundation of an intervention-induced relief from stuttering due to the fourth approach, such as for example the cognitive-behavior intervention (Menzies et al., 2016), would be highly interesting and an essential complement. Of greatest importance are future studies with children with persistent stuttering to test whether reorganization induces neurotypical brain functioning and to develop therapeutic strategies that protect children from relapse.

\section{Conclusion}

A one-year practice of fluency shaping speech techniques boosts the synchrony of spontaneous brain activity in core hubs of speech timing and voice control. Thus, successful speech restructuring shapes sensorimotor integration networks and is reflected in a longlasting, focal, neurofunctional reorganization. 
4. Fluent speech: neural basis of sensorimotor plasticity in developmental stuttering

\section{Acknowledgments}

This work was supported by the DFG (SO 429/4-1 to M.S). We thank Bettina Helten for the co-analysis of the speech samples, Michael Bartl for supporting the organization of the behavioral data, and Britta Perl and Ilona Pfahlert for assistance with the acquisition of the MRI data.

\section{Additional Information}

The author(s) declare no competing interests. 


\section{General Discussion}

In addition to disfluent speech, stuttering is associated with aberrant neuronal activity and performance during tasks of sensorimotor integration, speech planning, movement initiation and inhibition. Relevant brain structures within neuronal networks contributing to these processes have also been associated with trait and state stuttering (see Section 1.3). To separate these neuronal and cognitive processes and to investigate their contribution to the emergence of stuttering events is experimentally challenging. The aim of my thesis was to further the knowledge of trait and state stuttering by examining different aspects of the four theories described in Section 1.3.

In the first study, presented in Chapter 2, I investigated neuronal oscillations during the formation of the sensorimotor integration speech network, i.e. sensorimotor preparation, in trait and state stuttering. Normal sensorimotor preparation is a prerequisite for the subsequent functional retrieval of speech motor programs and is associated with a decrease in alpha and beta power. To separate sensorimotor preparation from any speech processing, I used a cue-target self-initiated reading paradigm. The cue stimulus indicated whether overt or covert reading was required after the linguistic target stimulus. Thus, the cue stimulus led to overt or covert speech intention. Alpha and beta EEG oscillations revealed that sensorimotor preparation was stronger during the intention of overt compared with covered speech in both groups. Further, neither trait nor state stuttering showed aberrant sensorimotor preparation: oscillatory activity was similar in AWS (adults with developmental stuttering) and FC (fluent controls), as well as in AWS before fluent and stuttered speech. However, AWS with more severe stuttering showed stronger beta power in the right-hemispheric frontal EEG. Thus, although trait and state stuttering yielded similar power decreases during sensorimotor preparation, an increase in stuttering severity was associated with higher inhibitory activity. 


\section{General Discussion}

In the second study, presented in Chapter 3, I investigated whether syllable frequency influenced speech fluency and accuracy in AWS similar to known effects in fluent speakers. High-frequency (i.e. automatized sequences of several phonemes) compared with lowfrequency (i.e. assembled phoneme by phoneme) syllables are associated with shorter speech planning time and better accuracy in fluent speakers (e.g. Laganaro \& Alario, 2006). Speech data was derived from the EEG experiment in Chapter 2, which had included selfinitiated reading of 300 pseudowords with different lengths (two-, three-, and four-syllabic). The 300 pseudowords consisted of two equally sized groups with either a high- or lowfrequency initial syllable. The results did not show the expected frequency effect in AWS. Contrarily, a trend for an inverted frequency effect was observed: $63 \%$ of AWS (12 out of 19) stuttered more on high-frequency than on low-frequency words. Although, this observation was not statistical significant, the reversed syllable frequency effect was confirmed by a significant interaction between group and syllable frequency showing that $42 \%$ of AWS (8 out of 19 ) were less accurate on high- than on low-frequency syllables, whereas $\mathrm{FC}$ responded in the expected pattern. Moreover, a significant interaction between syllable frequency and pseudoword length on response accuracy revealed that the execution of four-syllabic pseudowords overrode the reversed frequency error pattern in AWS. Thus, both groups made more errors on four-syllabic pseudowords with lowfrequency initial syllables. Last, increasing pseudoword length led to more stuttering events (in AWS) and errors (in AWS and FC) on initial syllables. To summarize, I conclude that increasing sequence length influences speech production in AWS and FC in a similar way, but a reversed syllable frequency effect distinguishes AWS from FC and may reflect an aberrant functioning of the cortico-basal ganglia circuit in AWS.

In the third study, presented in Chapter 4, I used a more global approach: Here, I investigated the influence of a stuttering intervention, which focuses on articulatory techniques (fluency shaping), on the rs-fMRI connectivity of four functional networks associated with stuttering. In addition, the influence of fluency shaping on white matter 


\section{General Discussion}

integrity of major speech tracts was examined. I compared functional and structural connectivity from AWS participating in the intervention with those of stuttering and healthy controls before and after the intervention. The stuttering intervention increased the functional connectivity in the sensorimotor integration network in stuttering patients. Further, the white matter integrity of the left SLF, an underlying white matter structure of the sensorimotor integration network, correlated positively with increased speech fluency after the intervention. In addition, stuttering patients with lower white matter integrity of the right FAT showed a better intervention outcome for the psycho-social assessment of the selfexperience with stuttering (OASES, Yaruss et al., 2016). Thus, successful stuttering intervention was associated with speech motor learning, which was shaped by structural and functional connectivity of the sensorimotor integration and the inhibitory control network. In the following, I first discuss these results in the frameworks of sensorimotor integration and inhibition. Furthermore, I outline future research questions.

\subsection{Sensorimotor integration - preventing or inducing stuttering events?}

As laid out in Chapter 1, the final process of speech preparation is the activation of internal models of feedforward sensory and motor control. One theory of deficient sensorimotor integration in stuttering suggests that stuttering occurs due to insufficiently activated or instable internal models (Max, 2004).

\subsubsection{Normal sensorimotor preparation in stuttering}

The preparation and retrieval of internal speech models are associated with a decrease in beta power over sensorimotor cortices (Jenson et al., 2019). This is accompanied by a decrease in alpha power indicating preparatory and actual sensory feedback processing (Jenson et al., 2019). Importantly, studies demonstrated that regions associated with internal models of motor and sensory programs, i.e. the sensorimotor integration network, 


\section{General Discussion}

are activated already during speech intention (Gehrig et al., 2012; Kell et al., 2011). Such results suggest that prior to the retrieval of a specific motor program relevant sensorimotor brain regions are prepared for upcoming speech processing. In Chapter 2, I showed that oscillatory modulation indicating sensorimotor preparation did not differ AWS from FC. Further, I found a similar sensorimotor preparation prior to the fluent and stuttered speech of AWS. However, during speech preparation and execution, where specific internal models are retrieved, studies have reported aberrant alpha and beta decreases in AWS (reduced decrease: Jenson et al., 2018; stronger decrease: Mersov et al., 2016; Mock et al., 2016). Our results could have two conceivable implications. First, as discussed in Chapter 2, a decrease in the signal to noise ratio, which possibly emerged due to the applied baseline normalization procedures and sensor level analyses, might have obscured differences between groups and fluency states. Second, it is possible that the trait and state stuttering differences in oscillatory modulation in sensorimotor regions are tightly linked to movement and linguistic context. In other words, sensorimotor preparation in AWS might have been normal during speech intention, as no concrete internal models were prepared and retrieved. Recent findings indicated a stronger pre-movement alpha and a reduced premovement beta power decrease prior to a reaching task with uncertain movement targets compared with a known target (Tzagarakis et al., 2015). Thus, the degree of pre-movement beta power decrease could represent the inverse uncertainty afforded to sensory prediction errors (Palmer et al., 2019). This view on beta power modulation over sensorimotor regions implicates that the stability of internal models is assessed during the planning process. During the speech intention task described in Chapter 2 the speech movement itself was unknown, and no internal models were retrieved. Taken together, the data implicate that the prerequisite for a functioning sensorimotor integration, i.e. the orienting process towards the task, does not contribute to trait stuttering. Furthermore, the retrieval of internal models and the computation of movement uncertainty are presumable factors, which are going to be discussed in the following. 


\section{General Discussion}

\subsubsection{Stability of internal models}

As described on the previous page, sensorimotor integration difficulties in stuttering might stem from insufficient activation of internal models or from unstable, presumably poorly acquired internal models.

In Chapter 3, we showed that high-frequency syllables, which, in fluent speakers, are less error prone, tended to induce more stuttering and response errors in AWS. We suggested that the reversed syllable frequency effect in AWS might reflect unstable motor programs as a result of impaired motor learning (e.g. Kim et al., 2020). In seeming contrast to impaired learning in AWS, our findings in Chapter 4 revealed that stuttering intervention led to increased connectivity between regions involved in successful speech motor adaption (Darainy et al., 2018) and with the cortical representations of internal speech motor and auditory programs (Guenther, 2016). Thus, AWS participating in a stuttering intervention were able to strengthen cortical internal models encompassing both motor and sensory speech programs. Two questions arise from this interpretation.

First, how can the contradicting findings of impaired sensorimotor learning in stuttering of previous studies and of ours be explained? Long-term practice and visual feedback might account for these different results. Most study results on impaired motor learning in persons with developmental stuttering are conducted in the course of one day (e.g. Kim et al., 2020) or maximally one week (Namasivayam \& van Lieshout, 2008). However, the effect of stuttering intervention in Chapter 4 was assessed at the end of one year during which participants had practiced almost daily the new speech technique. Therefore, our findings of increased connectivity indicating speech motor learning could have resulted from frequent long-term practice. In addition, Kim et al. (2020) reported that speech auditorymotor learning in AWS and CWS was more deficient than hand visuomotor learning. The use of visual feedback in the stuttering-intervention under study seems to have supported deficient speech auditory-motor learning in AWS. 


\section{General Discussion}

The second question pertains to the seemingly differing interpretation of results in Chapters 3 and 4. Although, high-frequency syllables are not learned explicitly, their frequent use in speech production is assumed to generate traversing motor programs of the underlying phoneme sequence (Segawa et al., 2015). The stuttering intervention under study however did not focus on the acquisition of new syllables but on the adaption of articulatory parameters to existing speech motor programs. Our findings seem to reflect this emphasis on articulatory shaping. We report increased connectivity within the sensorimotor integration network but not the cortico-basal ganglia network, i.e. articulatory convergence (Chapter 4), that includes the basal-ganglia as a relevant region of motor sequence learning (Doyon et al., 2009; Segawa et al., 2015). Thus, whereas AWS can learn sensorimotor adaption of specific phonemes, the stability of internal high-frequency models that are based on sequential motor learning might still be impaired. This interpretation favors theories on stuttering that consider a dysfunction of the cortico-basal ganglia circuit (Section 1.3.3 and 1.3.4). Specifically, elevated levels of dopamine within the striatum can account for stuttering events in initial syllable position by delaying the initiation of the planned motor program (Civier et al., 2013). In addition, a hyperdopaminergic level can lead to a higher speech error rate as competing motor programs are insufficiently inhibited (Chang \& Guenther, 2019). Those consequences together with the reduced stability of high-frequency syllables could lead to our observed reversed syllable frequency effect in AWS. I, therefore, conclude that internal model stability is likely not affected by a sensorimotor integration deficit, as this instability can be better accounted for by a dysfunction of the cortico-basal ganglia circuit, as suggested by the theory of aberrant initiation and inhibition in stuttering (Alm, 2004; Civier et al., 2013). However, internal model stability can be increased through sensorimotor learning during a long-term stuttering intervention. 


\section{General Discussion}

\subsubsection{Sequence length and sensorimotor integration}

Although, Max and colleagues (2004) did not account for the processing of sequence or utterance length within their framework of sensorimotor integration, recent neurophysiological findings on structures pertaining to the sensorimotor integration network foster such a discussion. These findings may help to explain the length effect reported in Chapter 3, as I will show in the following.

A positive correlation between white matter integrity of the SLF and verbal memory performance is reported for fluent adults (Koshiyama et al., 2020) but not for children (Krogsrud et al., 2018). In addition, during the maintenance and retrieval of sentences and pseudowords oscillatory power modulation over fronto-temporo-parietal regions of the sensorimotor integration network varies with the sequence length of the linguistic items (Gehrig et al., 2019; Herman et al., 2013). These findings implicate that an intact sensorimotor integration network plays a crucial role for the processing of sequence length. As summarized above, in Chapter 3 we reported for the initial syllable a robust effect of pseudoword length on stuttering and error probability in AWS but also on error probability in FC. This finding is in line with numerous studies, which report poorer speech performance (e.g. increase of stuttering, errors and reaction times) with increasing sequence length in AWS, CWS and also FC (e.g. Herman et al., 2013; Sasisekaran \& Weisberg, 2014; Smith et al., 2010). Important, however, is the notion that sequence length per se does not differentiate the response accuracy of stuttering persons and FC; both groups often perform similarly well, and differences are only found with increasing task difficulty (e.g. pseudowords with more than five syllables) (Byrd et al., 2012; Coalson et al., 2018; Sasisekaran \& Weisberg, 2014). Emerging group differences on very long sequences, might be associated with a decreased white matter integrity within the left SLF as it is shown for trait stuttering (Neef et al., 2015; Neef et al., 2018). In support of a sensorimotor integration deficit, I report that stuttering patients, who gain higher scores in speech fluency after intervention, have a stronger connectivity within the left SLF (Chapter 4). Further, I 


\section{General Discussion}

show that the aberrant frequency effect in AWS is overridden with increasing sequence length (Chapter 3). However, a deficient sensorimotor integration during phonological encoding cannot account for the emergence of stuttering events at the initial syllable position. Usually, in verbal memory tasks, the first presented items are reported with higher accuracy than subsequent items (Camos et al., 2017). The framework of dysfunctional speech initiation due to a deficient cortico-basal ganglia circuit can better account for the interaction between increasing sequence length and the emergence of stuttering events in the first syllable position (Guenther, 2016).

In summary, the results show that although the primary mechanism of stuttering is more likely to be explained in the context of a cortico-basal ganglia dysfunction, reduced sensorimotor integration, as indicated by altered white matter integrity, can influence and aggravate this dysfunction. In addition, individuals with a stronger connectivity within the sensorimotor integration network seem to profit more from fluency shaping techniques.

\subsection{The role of aberrant inhibition in stuttering}

A recently developed theory on stuttering, suggests that in addition to a left hemispheric impairment of brain structures, right hemispheric overactivity and increased structural connectivity might implicate an unspecific broad inhibition in stuttering (Neef et al., 2018). In the following, I discuss results of my thesis that show an association between beta power and stuttering severity within this framework.

\subsubsection{Higher inhibitory levels during sensorimotor preparation in severe stuttering}

Results in Chapter 2 revealed that AWS with more severe stuttering had stronger beta power levels, predominately in the right-hemispheric EEG, although the transient sensorimotor preparation in AWS was similar to FC. Increases in beta power in cortical regions and the subthalamic nucleus are observed simultaneously during tasks testing sensorimotor inhibition (Swann et al., 2009; Wessel et al., 2016) but also during non-motor 


\section{General Discussion}

cognitive tasks, in which participants suppressed the memorization of distraction items (Zavala et al., 2017). An EEG-TMS study showed that beta power over the right IFG increased in response to a NoGo cue (Picazio et al., 2014). In this study, the inhibitory pulse led to reduced excitability with a concomitant beta power increase in the left motor cortex. Hence, our finding is concordant with Mersov et al. (2016), who reported greater beta power over bilateral premotor regions (BA 6) prior to the occurrence of speech stimuli in AWS but not in FC. Thus, the results in Chapter 2 might indicate a stronger inhibition of sensorimotor regions during speech intention in AWS with more severe stuttering. This interpretation is in accord with TMS studies which reported decreased facilitation of the tongue motor cortex during rest in AWS (Neef et al., 2011).

Further support for interpreting right-hemispheric beta power increases as indicator for increased inhibitor levels, stems from the fact that the hyperdirect pathway, an inhibitory network, is associated with right-hemispheric regions. Specifically, the right IFG and the right SMA are interconnected through the right FAT (Dick et al., 2018), and both regions together with subthalamic nucleus form the hyperdirect inhibitory network (Brunenberg et al., 2012; Wiecki \& Frank, 2013). The right FAT has been reported to exhibit aberrant white matter integrity in AWS (Kronfeld-Duenias et al., 2016b; Neef et al., 2018). In Chapter 4, I describe how white matter integrity of the right FAT correlated negatively with postintervention scores in the overall assessment of the speaker's experience of stuttering scale (OASES). A similar correlation has been reported between the results in the quality of life subtest of the OASES and prolonged reaction times during a movement inhibition task in AWS (Treleaven \& Coalson, 2020). In addition, structural connectivity of the right FAT between the right IFG, pre-SMA and STN correlated positively with stuttering severity (Neef et al., 2018). Taken together, the studies described in Chapter 2 and Chapter 4 support the idea of an unbalanced right-hemispheric inhibitory network in stuttering, which could either reduce the readiness for encoding speech movements or actively inhibit movements. 


\section{General Discussion}

\subsubsection{Influence of network inhibition on the retrieval of internal models}

It is interesting to reflect on whether increased inhibitory levels might interfere with language processing. From my view, this is of high relevance, as the neurophysiological implications of an increased inhibitory network in stuttering are not yet clear (for a first synthesis see Metzger et al., 2018).

Beta power oscillations during movement intention have been reported to reflect performance of movement execution. In a finger tapping task, intending fast movements significantly influenced the decrease of beta power and correlated positively with reaction times (Pastötter et al., 2012). Thus, one could speculate that increased beta power during speech intention interfered more with the retrieval of speech motor programs, where a stronger reduction of beta power amplitude is required. In other words, the longer the peakto-peak distance between the amplitude of beta power increase and decrease, the more susceptible to inference is the retrieval of speech motor programs.

A stronger beta power decrease is observed prior to high-frequency words (Grabner et al., 2007) and motor learning (Pollok et al., 2014; Pollok et al., 2015; for contradicting findings see Espenhahn et al., 2019; Gehringer et al., 2018). Relating to speech, high-frequency syllables are well learned (Bohland et al., 2010) and produced with shorter reaction times and greater accuracy (Laganaro \& Alario, 2006). In fact, in Chapter 3, I showed that the retrieval and production of high-frequency syllables in the initial word position had a detrimental effect on stuttering probability and response accuracy in AWS. However, to my best knowledge it is yet unclear whether AWS differ in their modulation of beta power frequency relating to syllable, phoneme or non-speech movement frequency.

\subsubsection{Testing the influence of right-hemispheric inhibition}

In the previous section, I discussed how increased inhibition could influence the retrieval of motor programs in connection with syllable frequency. One possibility would be to examine the correlation between beta power modulation during speech intention and speech 


\section{General Discussion}

preparation while controlling for frequency effects. In addition, the inhibition of the right hemisphere with TMS might help to examine if there is a relationship between increased inhibitory levels and the retrieval of internal models in stuttering. In fact, two recent studies have provided the first evidence that inhibitory TMS over the right IFG decreased the percentage of stuttered syllables/moras during reading (Tezel-Bayraktaroglu et al., 2020; Yada et al., 2019). Interestingly, inhibitory low frequency repetitive TMS increased stuttering severity during spontaneous speech (Tezel-Bayraktaroglu et al., 2020), indicating that additional neuronal processes must be accounted for spontaneous speech.

In addition, a clear understanding of neurochemical mechanisms underlying beta power modulation in stuttering might advance the study of inhibitory processes.

Inference could be taken from other neurological disorders such as Parkinson's disease and Tourette syndrome, which are also associated with basal ganglia dysfunction (Alm, 2004) and show increased beta power during rest, movement execution and inhibition (Jenkinson \& Brown, 2011; Niccolai et al., 2016). However, many questions still arise when comparing results on higher beta power levels in Parkinson's disease with stuttering. In Parkinson's disease higher beta power levels are thought to emerge due to a lower concentration of dopamine in the striatum and increased connectivity to and from the STN (Jenkinson \& Brown, 2011; Marreiros et al., 2013). Conversely, increased dopamine levels are reported in developmental stuttering with positive responses to drugs inhibiting the activity of dopamine (Maguire et al., 2020; Wu et al., 1997). In addition, a recent study reported a positive correlation between stuttering severity and the substantia nigra, a region that supplies the striatum with dopamine, during movement preparation (Metzger et al., 2018). Further, deep brain stimulation of the STN in patients with Parkinson's disease that lowers the increased beta power can lead to an aggravation or reemergence of stuttering (Burghaus et al., 2006; Toft \& Dietrichs, 2011; but see Walker et al., 2009 for a report of stuttering decrease after stimulation). Beta oscillations can be generated in the basal ganglia but also at a cortical level (Brittain \& Brown, 2014; Jensen et al., 2005). Further, 


\section{General Discussion}

beta and gamma frequencies are more dependent on an intact structural white matter integrity than lower frequencies, such as alpha (Chu et al., 2015). Thus, the relationship between the observed cortical beta modulations in stuttering might follow another coupling with basal ganglia oscillatory activity than in Parkinson's disease. One suggestion is that a greater cortical beta power might reflect a compensatory mechanism in stuttering that emerges due to decreased beta power in the basal ganglia (Etchell et al., 2014).

In effect, my results highlight the need to include the right cortico-subthalamic nucleus circuit in considerations of stuttering. This suggestion is supported by findings of simultaneous oscillatory activity in the sensorimotor cortex and the STN during speech production (Chrabaszcz et al., 2019), and the engagement of bilateral IFG and STN during movement error processing (Steele et al., 2014). Future studies are needed to elucidate the neurochemical processes relevant for stronger beta power in AWS, their influence on beta power decrease during movement preparation and execution, and their coupling with other oscillatory frequency bands in trait and state stuttering.

\subsection{Conclusion and Outlook}

In conclusion, the results of this thesis show that processes of sensorimotor integration, speech initiation and inhibition, i.e. processes of phonological and phonetic encoding, jointly contribute to trait and state stuttering. This is in line with recent suggestions to treat stuttering as a system-level disorder (Chang \& Guenther, 2019). The results emphasize the need to consider brain activity during speech intention, as well as length and frequency effects as influential factors on neural speech preparation. To further unravel the mechanisms of stuttering emergence, future studies are required to elaborate the interaction between the presented brain networks, and also other networks, e.g. the attention network, which have been associated with stuttering (Chang, Angstadt et al., 2018). For this goal, methods with high temporal and spatial resolution are needed. One promising avenue is the investigation of neural oscillations as an indicator of the varying 


\section{General Discussion}

cognitive states. In addition, while the results of this thesis require in-depth cross-validation, it is tempting to speculate that performance on pseudoword repetition tasks, measures of white matter density or categorizations by genetic mutations (e.g. Frigerio-Domingues et al., 2019) could indicate the success of sensorimotor learning in specific stuttering interventions. Such knowledge then could be used to adapt stuttering interventions to individual neurophysiological factors by applying for example additional individualized transcranial brain stimulation. 
6. References

\section{References}

Ackermann, H., Hertrich, I., Ziegler, W., Bitzer, M., \& Bien, S. (1996). Acquired dysfluencies following infarction of the left mesiofrontal cortex. Aphasiology, 10(4), 409-417. https://doi.org/10.1080/02687039608248420

Alario, F.-X., Costa, A., \& Caramazza, A. (2002a). Frequency effects in noun phrase production: Implications for models of lexical access. Language and Cognitive Processes(17), Article 3, 299-319.

Alario, F.-X., Costa, A., \& Caramazza, A. (2002b). Hedging one's bets too much? A reply to Levelt (2002). Language and Cognitive Processes(17), Article 6, 673-682.

Albert, N. B., Robertson, E. M., \& Miall, R. C. (2009). The resting human brain and motor learning. Current Biology : CB, 19(12), 1023-1027. https://doi.org/10.1016/j.cub.2009.04.028

Alegre, M., Imirizaldu, L., Valencia, M., Iriarte, J., Arcocha, J., \& Artieda, J. (2006). Alpha and beta changes in cortical oscillatory activity in a go/no go randomly-delayedresponse choice reaction time paradigm. Clinical Neurophysiology, 117(1), 16-25. https://doi.org/10.1016/j.clinph.2005.08.030

Alm, P. A. (2004). Stuttering and the basal ganglia circuits: A critical review of possible relations. Journal of Communication Disorders, 37(4), 325-369. https://doi.org/10.1016/j.jcomdis.2004.03.001

Alm, P. A. (2005). On the causal mechanisms of stuttering [Dissertation]. Lund University, Lund.

Al-Tamimi, F., Khamaiseh, Z., \& Howell, P. (2013). Phonetic complexity and stuttering in Arabic. Clinical Linguistics \& Phonetics, 27(12), 874-887.

Anderson, J. D. (2007). Phonological Neighborhood and Word Frequency Effects in the Stuttered Disfluencies of Children Who Stutter. Journal of Speech, Language, and 
6. References

Hearing Research : JSLHR, 50(1), 229-247. https://doi.org/10.1044/1092$4388(2007 / 018)$

Andersson Jesper LR, Jenkinson, M., \& Smith, S. (2007). Non-linear registration, aka Spatial normalisation: FMRIB Technial Report TR07JA2.

Andreatta, R. D., Stemple, J. C., Joshi, A., \& Jiang, Y. (2010). Task-related differences in temporo-parietal cortical activation during human phonatory behaviors. Neuroscience Letters, 484(1), 51-55. https://doi.org/10.1016/j.neulet.2010.08.017

Andrews-Hanna, J. R., Snyder, A. Z., Vincent, J. L., Lustig, C., Head, D., Raichle, M. E., \& Buckner, R. L. (2007). Disruption of large-scale brain systems in advanced aging. Neuron, 56(5), 924-935. https://doi.org/10.1016/j.neuron.2007.10.038

Aron, A. R., \& Poldrack, R. A. (2006). Cortical and subcortical contributions to Stop signal response inhibition: Role of the subthalamic nucleus. The Journal of Neuroscience : The Official Journal of the Society for Neuroscience, 26(9), 2424-2433. https://doi.org/10.1523/JNEUROSCI.4682-05.2006

Au-Yeung, J., Howell, P., \& Pilgrim, L. (1998). Phonological words and stuttering on function words. Journal of Speech, Language, and Hearing Research : JSLHR(41), $1019-1030$.

Baayen, R. H., Davidson, D. J., \& Bates, D. M. (2008). Mixed-effects modeling with crossed random effects for subjects and items. Journal of Memory and Language, 59(4), 390-412. https://doi.org/10.1016/j.jml.2007.12.005

Baayen, R.H., Piepenbrock, R., \& Gulikers, L. (1996). The CELEX Lexical Database: (cdrom). Linguistic Data Consortium.

Barr, D. J., Levy, R., Scheepers, C., \& Tily, H. J. (2013). Random effects structure for confirmatory hypothesis testing: Keep it maximal. Journal of Memory and Language, 68(3). https://doi.org/10.1016/j.jml.2012.11.001 


\section{References}

Bates, D., Kliegl, R., Vasishth, S., \& Baayen, R.H. (2018). Parsimonious Mixed Models. arXiv.

Bates, D., Mächler, M., Bolker, B., \& Walker, S. (2015). Fitting Linear Mixed-Effects Models Using Ime4. Journal of Statistical Software, 67(1). https://doi.org/10.18637/jss.v067.i01

Baxter, S., Johnson, M., Blank, L., Cantrell, A., Brumfitt, S., Enderby, P., \& Goyder, E. (2016). Non-pharmacological treatments for stuttering in children and adults: A systematic review and evaluation of clinical effectiveness, and exploration of barriers to successful outcomes. Health Technology Assessment (Winchester, England), 20(2), 1 302, v-vi. https://doi.org/10.3310/hta20020

Beal, D. S., Gracco, V. L., Brettschneider, J., Kroll, R. M., \& Nil, L. F. de (2013). A voxelbased morphometry (VBM) analysis of regional grey and white matter volume abnormalities within the speech production network of children who stutter. Cortex; a Journal Devoted to the Study of the Nervous System and Behavior, 49(8), 2151-2161. https://doi.org/10.1016/j.cortex.2012.08.013

Beal, D. S., Lerch, J. P., Cameron, B., Henderson, R., Gracco, V. L., \& Nil, L. F. de (2015). The trajectory of gray matter development in Broca's area is abnormal in people who stutter. Frontiers in Human Neuroscience, 9, 89.

https://doi.org/10.3389/fnhum.2015.00089

Beck, A. T., Steer, R. A., \& Brown, G. (1996) [Beck Depression Inventory®-II] Beck Depression Inventory®-II: (BDI@-II). Pearson.

Behrens, T. E. J., Berg, H. J., Jbabdi, S., Rushworth, M. F. S., \& Woolrich, M. W. (2007). Probabilistic diffusion tractography with multiple fibre orientations: What can we gain? Neurolmage, 34(1), 144-155. https://doi.org/10.1016/j.neuroimage.2006.09.018

Behroozmand, R., Shebek, R., Hansen, D. R., Oya, H., Robin, D. A., Howard, M. A. 3., \& Greenlee, J. D. W. (2015). Sensory-motor networks involved in speech production and 


\section{References}

motor control: An fMRI study. Neurolmage, 109, 418-428.

https://doi.org/10.1016/j.neuroimage.2015.01.040

Beilby, J. M., Byrnes, M. L., \& Yaruss, J. S. (2012). The Impact of a Stuttering Disorder on Western Australian Children and Adolescents. Perspectives on Fluency and Fluency Disorders, 22(2), 51-62. https://doi.org/10.1044/ffd22.2.51

Belyk, M., Kraft, S. J., \& Brown, S. (2015). Stuttering as a trait or state - an ALE metaanalysis of neuroimaging studies. The European Journal of Neuroscience, 41(2), 275284. https://doi.org/10.1111/ejn.12765

Belyk, M., Kraft, S. J., \& Brown, S. (2017). Stuttering as a trait or a state revisited: Motor system involvement in persistent developmental stuttering: Motor system involvement in persistent developmental stuttering. The European Journal of Neuroscience, 45(4), 622-624. https://doi.org/10.1111/ejn.13512

Benwell, C. S. Y., London, R. E., Tagliabue, C. F., Veniero, D., Gross, J., Keitel, C., \& Thut, G. (2019). Frequency and power of human alpha oscillations drift systematically with time-on-task. Neurolmage, 192, 101-114. https://doi.org/10.1016/j.neuroimage.2019.02.067

Blood, G. W., \& Blood, I. M. (2004). Bullying in Adolescents Who Stutter: Communicative Competence and Self-Esteem. Contemporary Issues in Communication Science and Disorders(31), 69-79.

Bloodstein, O. (1975). Stuttering as tension and fragmentation. In J. Eisenson (Ed.), Stuttering: A second symposium (pp. 1-96). Harper \& Row.

Bloodstein, O., \& Ratner, N. B. (2008). A handbook on stuttering (6. ed.). Delmar/Cengage Learning.

Blumgart, E., Tran, Y., Yaruss, J. S., \& Craig, A. (2012). Australian normative data for the Overall Assessment of the Speaker's Experience of Stuttering. Journal of Fluency Disorders, 37(2), 83-90. https://doi.org/10.1016/j.jfludis.2011.12.002 


\section{References}

Bohland, J. W., Bullock, D., \& Guenther, F. H. (2010). Neural Representations and Mechanisms for the performance of simple speech sequences. Journal of Cognitive Neuroscience(22), Article 7, 1504-1529.

Bothe, A. K., Davidow, J. H., Bramlett, R. E., Franic, D. M., \& Ingham, R. J. (2006). Stuttering Treatment Research 1970-2005: II. Systematic Review Incorporating Trial Quality Assessment of Pharmacological Approaches. American Journal of SpeechLanguage Pathology, 15(4), 342-352.

Bothe, A. K., Davidow, J. H., Bramlett, R. E., \& Ingham, R. J. (2006). Stuttering Treatment Research 1970-2005: I. Systematic Review Incorporating Trial Quality Assessment of Behavioral, Cognitive, and Related Approaches. American Journal of SpeechLanguage Pathology, 15(4), 321-341. https://doi.org/10.1044/1058-0360(2006/031)

Bouhali, F., Bézagu, Z., Dehaene, S., \& Cohen, L. (2019). A mesial-to-lateral dissociation for orthographic processing in the visual cortex. Proceedings of the National Academy of Sciences of the United States of America, 116(43), 21936-21946. https://doi.org/10.1073/pnas.1904184116

Bowers, A., Bowers, L. M., Hudock, D., \& Ramsdell-Hudock, H. L. (2018). Phonological working memory in developmental stuttering: Potential insights from the neurobiology of language and cognition. Journal of Fluency Disorders, 58, 94-117. https://doi.org/10.1016/j.jfludis.2018.08.006

Brinkman, L., Stolk, A., Dijkerman, H. C., Lange, F. P. de, \& Toni, I. (2014). Distinct roles for alpha- and beta-band oscillations during mental simulation of goal-directed actions. The Journal of Neuroscience : The Official Journal of the Society for Neuroscience, 34(44), 14783-14792. https://doi.org/10.1523/JNEUROSCI.2039-14.2014

Brittain, J.-S., \& Brown, P. (2014). Oscillations and the basal ganglia: Motor control and beyond. Neurolmage, 85 Pt 2, 637-647. https://doi.org/10.1016/j.neuroimage.2013.05.084 


\section{References}

Brocklehurst, P. H., Lickley, R. J., \& Corley, M. (2013). Revisiting Bloodstein's Anticipatory Struggle Hypothesis from a psycholinguistic perspective: A Variable Release Threshold hypothesis of stuttering. Journal of Communication Disorders, 46(3), 217-237. https://doi.org/10.1016/j.jcomdis.2013.04.002

Brown, S. F. (1938a). A further study of stuttering in relation to various speech sounds*. Quarterly Journal of Speech, 24(3), 390-397. https://doi.org/10.1080/00335633809380385

Brown, S. F. (1938b). Stuttering with relation to word accent and word position. The Journal of Abnormal and Social Psychology.

Brown, S. F. (1945). The loci of stutterings in the speech sequence. Journal of Speech Disorders(10), 181-192.

Brown, S., Ingham, R. J., Ingham, J. C., Laird, A. R., \& Fox, P. T. (2005). Stuttered and fluent speech production: An ALE meta-analysis of functional neuroimaging studies. Human Brain Mapping, 25(1), 105-117. https://doi.org/10.1002/hbm.20140

Brown, S., Ngan, E., \& Liotti, M. (2008). A larynx area in the human motor cortex. Cerebral Cortex (New York, N.Y. : 1991), 18(4), 837-845. https://doi.org/10.1093/cercor/bhm131

Brunenberg, E. J. L., Moeskops, P., Backes, W. H., Pollo, C., Cammoun, L., Vilanova, A., Janssen, M. L. F., Visser-Vandewalle, V. E. R. M., ter Haar Romeny, B. M., Thiran, J.P., \& Platel, B. (2012). Structural and resting state functional connectivity of the subthalamic nucleus: Identification of motor STN parts and the hyperdirect pathway. PloS One, 7(6), e39061. https://doi.org/10.1371/journal.pone.0039061

Büchel, C., \& Sommer, M. (2004). What causes stuttering? PloS Biology, 2(2), E46. https://doi.org/10.1371/journal.pbio.0020046 


\section{References}

Budde, K. S., Barron, D. S., \& Fox, P. T. (2014). Stuttering, induced fluency, and natural fluency: A hierarchical series of activation likelihood estimation meta-analyses. Brain and Language, 139, 99-107. https://doi.org/10.1016/j.bandl.2014.10.002

Burghaus, L., Hilker, R., Thiel, A., Galldiks, N., Lehnhardt, F. G., Zaro-Weber, O., Sturm, V., \& Heiss, W.-D. (2006). Deep brain stimulation of the subthalamic nucleus reversibly deteriorates stuttering in advanced Parkinson's disease. Journal of Neural Transmission (Vienna, Austria : 1996), 113(5), 625-631. https://doi.org/10.1007/s00702-005-0341-1

Bürki, A., Cheneval, P. P., \& Laganaro, M. (2015). Do speakers have access to a mental syllabary? Erp comparison of high frequency and novel syllable production. Brain and Language, 150, 90-102. https://doi.org/10.1016/j.bandl.2015.08.006

Bürki, A., Viebahn, M., \& Gafos, A. (2020). Plasticity and transfer in the sound system: exposure to syllables in production or perception changes their subsequent production. Language, Cognition and Neuroscience, 1-23. https://doi.org/10.1080/23273798.2020.1782445

Busan, P. (2020). Developmental stuttering and the role of the supplementary motor cortex. Journal of Fluency Disorders, 64, 105763.

https://doi.org/10.1016/j.jfludis.2020.105763

Buzsáki, G. (2006). Brain oscillations. Current Biology, 23(22), R983-R985. https://doi.org/10.1016/j.cub.2013.10.040

Buzsáki, G., Logothetis, N., \& Singer, W. (2013). Scaling brain size, keeping timing: Evolutionary preservation of brain rhythms. Neuron, 80(3), 751-764. https://doi.org/10.1016/j.neuron.2013.10.002

Byrd, C. T., McGill, M., \& Usler, E. (2015). Nonword repetition and phoneme elision in adults who do and do not stutter: Vocal versus nonvocal performance differences. Journal of Fluency Disorders, 44, 17-31. https://doi.org/10.1016/j.jfludis.2015.01.004 


\section{References}

Byrd, C. T., Vallely, M., Anderson, J. D., \& Sussman, H. (2012). Nonword repetition and phoneme elision in adults who do and do not stutter. Journal of Fluency Disorders, 37(3), 188-201. https://doi.org/10.1016/j.jfludis.2012.03.003

Cai, S., Beal, D. S., Ghosh, S. S., Guenther, F. H., \& Perkell, J. S. (2014). Impaired timing adjustments in response to time-varying auditory perturbation during connected speech production in persons who stutter. Brain and Language, 129, 24-29. https://doi.org/10.1016/j.bandl.2014.01.002

Cai, S., Beal, D. S., Ghosh, S. S., Tiede, M. K., Guenther, F. H., \& Perkell, J. S. (2012). Weak responses to auditory feedback perturbation during articulation in persons who stutter: Evidence for abnormal auditory-motor transformation. PloS One, 7(7), e41830. https://doi.org/10.1371/journal.pone.0041830

Cai, S., Tourville, J. A., Beal, D. S., Perkell, J. S., Guenther, F. H., \& Ghosh, S. S. (2014). Diffusion imaging of cerebral white matter in persons who stutter: Evidence for network-level anomalies. Frontiers in Human Neuroscience, 8, 54.

https://doi.org/10.3389/fnhum.2014.00054

Calmels, C. (2020). Neural correlates of motor expertise: Extensive motor training and cortical changes. Brain Research, 1739, 146323.

https://doi.org/10.1016/j.brainres.2019.146323

Camos, V., Lagner, P., \& Loaiza, V. M. (2017). Maintenance of item and order information in verbal working memory. Memory (Hove, England), 25(8), 953-968. https://doi.org/10.1080/09658211.2016.1237654

Carreiras, M., \& Perea, M. (2004). Naming pseudowords in Spanish: Effects of syllable frequency. Brain and Language, 90(1-3), 393-400.

Chang, S.-E., Angstadt, M., Chow, H. M., Etchell, A. C., Garnett, E. O., Choo, A. L., Kessler, D., Welsh, R. C., \& Sripada, C. (2018). Anomalous network architecture of the 


\section{References}

resting brain in children who stutter. Journal of Fluency Disorders, 55, 46-67. https://doi.org/10.1016/j.jfludis.2017.01.002

Chang, S.-E., Chow, H. M., Wieland, E. A., \& McAuley, J. D. (2016). Relation between functional connectivity and rhythm discrimination in children who do and do not stutter. Neurolmage. Clinical, 12, 442-450. https://doi.org/10.1016/j.nicl.2016.08.021

Chang, S.-E., Erickson, K. I., Ambrose, N. G., Hasegawa-Johnson, M. A., \& Ludlow, C. L. (2008). Brain anatomy differences in childhood stuttering. Neurolmage, 39(3), 13331344. https://doi.org/10.1016/j.neuroimage.2007.09.067

Chang, S.-E., Garnett, E. O., Etchell, A., \& Chow, H. M. (2018). Functional and Neuroanatomical Bases of Developmental Stuttering: Current Insights. The Neuroscientist : A Review Journal Bringing Neurobiology, Neurology and Psychiatry, 1073858418803594. https://doi.org/10.1177/1073858418803594

Chang, S.-E., \& Guenther, F. H. (2019). Involvement of the Cortico-Basal GangliaThalamocortical Loop in Developmental Stuttering. Frontiers in Psychology, 10, 3088. https://doi.org/10.3389/fpsyg.2019.03088

Chang, S.-E., \& Zhu, D. C. (2013). Neural network connectivity differences in children who stutter. Brain : A Journal of Neurology, 136(Pt 12), 3709-3726. https://doi.org/10.1093/brain/awt275

Chang, S.-E., Zhu, D. C., Choo, A. L., \& Angstadt, M. (2015). White matter neuroanatomical differences in young children who stutter. Brain : A Journal of Neurology, 138(Pt 3), 694-711. https://doi.org/10.1093/brain/awu400

Cholin, J., Dell, G. S., \& Levelt, W. J. M. (2011). Planning and articulation in incremental word production: Syllable-frequency effects in English. Journal of Experimental Psychology. Learning, Memory, and Cognition, 37(1), 109-122.

https://doi.org/10.1037/a0021322 


\section{References}

Cholin, J., \& Levelt, W. J. M. (2009). Effects of syllable preparation and syllable frequency in speech production: Further evidence for syllabic units at a post-lexical level. Language and Cognitive Processes, 24(5), 662-684. https://doi.org/10.1080/01690960802348852

Cholin, J., Levelt, W. J. M., \& Schiller, N. O. (2006). Effects of syllable frequency in speech production. Cognition, 99(2), 205-235. https://doi.org/10.1016/j.cognition.2005.01.009

Chrabaszcz, A., Neumann, W.-J., Stretcu, O., Lipski, W. J., Bush, A., Dastolfo-Hromack, C. A., Wang, D., Crammond, D. J., Shaiman, S., Dickey, M. W., Holt, L. L., Turner, R. S., Fiez, J. A., \& Richardson, R. M. (2019). Subthalamic Nucleus and Sensorimotor Cortex Activity During Speech Production. The Journal of Neuroscience : The Official Journal of the Society for Neuroscience, 39(14), 2698-2708.

https://doi.org/10.1523/JNEUROSCI.2842-18.2019

Chu, C. J., Tanaka, N., Diaz, J., Edlow, B. L., Wu, O., Hämäläinen, M., Stufflebeam, S., Cash, S. S., \& Kramer, M. A. (2015). Eeg functional connectivity is partially predicted by underlying white matter connectivity. Neurolmage, 108, 23-33. https://doi.org/10.1016/j.neuroimage.2014.12.033

Civier, O., Bullock, D., Max, L., \& Guenther, F. H. (2013). Computational modeling of stuttering caused by impairments in a basal ganglia thalamo-cortical circuit involved in syllable selection and initiation. Brain and Language, 126(3), 263-278. https://doi.org/10.1016/j.bandl.2013.05.016

Civier, O., Tasko, S. M., \& Guenther, F. H. (2010). Overreliance on auditory feedback may lead to sound/syllable repetitions: Simulations of stuttering and fluency-inducing conditions with a neural model of speech production. Journal of Fluency Disorders, 35(3), 246-279. https://doi.org/10.1016/j.jfludis.2010.05.002 


\section{References}

Clos, M., Amunts, K., Laird, A. R., Fox, P. T., \& Eickhoff, S. B. (2013). Tackling the multifunctional nature of Broca's region meta-analytically: Co-activation-based parcellation of area 44. Neurolmage, 83, 174-188.

https://doi.org/10.1016/j.neuroimage.2013.06.041

Coalson, G. A., \& Byrd, C. T. (2017). Nonword repetition in adults who stutter: The effects of stimuli stress and auditory-orthographic cues. PloS One, 12(11), e0188111. https://doi.org/10.1371/journal.pone.0188111

Coalson, G. A., Byrd, C. T., \& Davis, B. L. (2012). The influence of phonetic complexity on stuttered speech. Clinical Linguistics \& Phonetics, 26(7), 646-659. https://doi.org/10.3109/02699206.2012.682696

Coalson, G. A., Byrd, C. T., Treleaven, S. B., \& Dang, L. (2018). Segmental and metrical complexity during non-word repetition in adults who stutter. Clinical Linguistics \& Phonetics, 1-23. https://doi.org/10.1080/02699206.2018.1504988

Coderre, E. L., Chernenok, M., Gordon, B., \& Ledoux, K. (2017). Linguistic and NonLinguistic Semantic Processing in Individuals with Autism Spectrum Disorders: An ERP Study. Journal of Autism and Developmental Disorders, 47(3), 795-812. https://doi.org/10.1007/s10803-016-2985-0

Cohen, M. X. (2014). Analyzing neural time series data: Theory and practice. Issues in clinical and cognitive neuropsychology. The MIT Press.

Connally, E. L., Ward, D., Howell, P., \& Watkins, K. E. (2014). Disrupted white matter in language and motor tracts in developmental stuttering. Brain and Language, 131, 2535. https://doi.org/10.1016/j.bandl.2013.05.013

Craig, A. (1998). Relapse following treatment for stuttering:: a critical review and correlative data. Journal of Fluency Disorders(23), 1-30. 


\section{References}

Craig, A., Blumgart, E., \& Tran, Y. (2009). The impact of stuttering on the quality of life in adults who stutter. Journal of Fluency Disorders, 34(2), 61-71.

https://doi.org/10.1016/j.jludis.2009.05.002

Craig, A., Hancock, K., Tran, Y., Craig, M., \& Peters, K. (2002). Epidemiology of stuttering in the community across the entire life span. Journal of Speech, Language and Hearing Research(45), 1097-1105.

Croot, K., Lalas, G., Biedermann, B., Rastle, K., Jones, K., \& Cholin, J. (2017). Syllable frequency effects in immediate but not delayed syllable naming in English. Language, Cognition and Neuroscience, 32(9), 1119-1132.

https://doi.org/10.1080/23273798.2017.1284340

Croot, K., Patterson, K., \& Hodges, J. R. (1998). Single Word Production in Nonfluent Progressive Aphasia. Brain and Language(61), 226-273.

Cykowski, M. D., Fox, P. T., Ingham, R. J., Ingham, J. C., \& Robin, D. A. (2010). A study of the reproducibility and etiology of diffusion anisotropy differences in developmental stuttering: A potential role for impaired myelination. Neurolmage, 52(4), 1495-1504. https://doi.org/10.1016/j.neuroimage.2010.05.011

Daliri, A., \& Max, L. (2015). Electrophysiological evidence for a general auditory prediction deficit in adults who stutter. Brain and Language, 150, 37-44. https://doi.org/10.1016/j.bandl.2015.08.008

Daliri, A., \& Max, L. (2018). Stuttering adults' lack of pre-speech auditory modulation normalizes when speaking with delayed auditory feedback. Cortex; a Journal Devoted to the Study of the Nervous System and Behavior, 99, 55-68.

https://doi.org/10.1016/j.cortex.2017.10.019

Damian, M. F., \& Dumay, N. (2007). Time pressure and phonological advance planning in spoken production 污. Journal of Memory and Language, 57(2), 195-209. https://doi.org/10.1016/j.jml.2006.11.001 


\section{References}

Darainy, M., Vahdat, S., \& Ostry, D. J. (2018). Neural Basis of Sensorimotor Plasticity in Speech Motor Adaptation. Cerebral Cortex (New York, N.Y. : 1991). Advance online publication. https://doi.org/10.1093/cercor/bhy153

Davis, S., Howell, P., \& Cooke, F. (2002). Sociodynamic relationships between children who stutter and their non-stuttering classmates. Journal of Child Psychology and Psychiatry, and Allied Disciplines, 43(7), 939-947. https://doi.org/10.1111/14697610.00093

Deco, G., \& Corbetta, M. (2011). The dynamical balance of the brain at rest. The Neuroscientist : A Review Journal Bringing Neurobiology, Neurology and Psychiatry, 17(1), 107-123. https://doi.org/10.1177/1073858409354384

Deco, G., Jirsa, V. K., \& McIntosh, A. R. (2011). Emerging concepts for the dynamical organization of resting-state activity in the brain. Nature Reviews. Neuroscience, 12(1), 43-56. https://doi.org/10.1038/nrn2961

Deco, G., Ponce-Alvarez, A., Mantini, D., Romani, G. L., Hagmann, P., \& Corbetta, M. (2013). Resting-state functional connectivity emerges from structurally and dynamically shaped slow linear fluctuations. The Journal of Neuroscience : The Official Journal of the Society for Neuroscience, 33(27), 11239-11252.

https://doi.org/10.1523/JNEUROSCI.1091-13.2013

Dell, G. S., Cornell, J., \& Govindjee, A. (1993). Structure and content in language production: A theory of frame constraints in phonological speech errors. Cognitive Science, 17(2), 149-195. https://doi.org/10.1016/0364-0213(93)90010-6

Dick, A. S., Garic, D., Graziano, P., \& Tremblay, P. (2018). The frontal aslant tract (FAT) and its role in speech, language and executive function. https://doi.org/10.1101/249912

Dosenbach, N. U. F., Visscher, K. M., Palmer, E. D., Miezin, F. M., Wenger, K. K., Kang, H. C., Burgund, E. D., Grimes, A. L., Schlaggar, B. L., \& Petersen, S. E. (2006). A core 
6. References

system for the implementation of task sets. Neuron, 50(5), 799-812.

https://doi.org/10.1016/j.neuron.2006.04.031

Doyon, J., Bellec, P., Amsel, R., Penhune, V., Monchi, O., Carrier, J., Lehéricy, S., \& Benali, H. (2009). Contributions of the basal ganglia and functionally related brain structures to motor learning. Behavioural Brain Research, 199(1), 61-75. https://doi.org/10.1016/j.bbr.2008.11.012

Drijvers, L., Özyürek, A., \& Jensen, O. (2018). Hearing and seeing meaning in noise: Alpha, beta, and gamma oscillations predict gestural enhancement of degraded speech comprehension. Human Brain Mapping, 39(5), 2075-2087. https://doi.org/10.1002/hbm.23987

Dworzynski, K., \& Howell, P. (2004). Predicting stuttering from phonetic complexity in German. Journal of Fluency Disorders, 29(2), 149-173.

https://doi.org/10.1016/j.jfludis.2004.03.001

Dworzynski, K., Remington, A., Rijsdijk, F., Howell, P., \& Plomin, R. (2007). Genetic Etiology in Cases of Recovered and Persistent Stuttering in an Unselected, Longitudinal Sample of Young Twins. American Journal of Speech-Language Pathology, 16(2), 169-178. https://doi.org/10.1044/1058-0360(2007/021)

Eggers, K., De Nil, L. F., \& van den Bergh, B. R. H. (2013). Inhibitory control in childhood stuttering. Journal of Fluency Disorders, 38(1), 1-13.

https://doi.org/10.1016/j.jfludis.2012.10.001

Eggers, K., \& Jansson-Verkasalo, E. (2017). Auditory Attentional Set-Shifting and Inhibition in Children Who Stutter. Journal of Speech, Language, and Hearing Research : JSLHR, 60(11), 3159-3170. https://doi.org/10.1044/2017_JSLHR-S-160096

ELAN (Version 5.8) [Computer software]. (2018). Max Planck Institute for Psycholinguistics, The Language Archive. Nijmegen. https://archive.mpi.nl/tla/elan 


\section{References}

Engel, A. K., \& Fries, P. (2010). Beta-band oscillations--signalling the status quo? Current Opinion in Neurobiology, 20(2), 156-165. https://doi.org/10.1016/j.conb.2010.02.015

Ergenoglu, T., Demiralp, T., Bayraktaroglu, Z., Ergen, M., Beydagi, H., \& Uresin, Y. (2004). Alpha rhythm of the EEG modulates visual detection performance in humans. Brain Research. Cognitive Brain Research, 20(3), 376-383. https://doi.org/10.1016/j.cogbrainres.2004.03.009

Espenhahn, S., van Wijk, B. C. M., Rossiter, H. E., Berker, A. O. de, Redman, N. D., Rondina, J., Diedrichsen, J., \& Ward, N. S. (2019). Cortical beta oscillations are associated with motor performance following visuomotor learning. Neurolmage, 195, 340-353. https://doi.org/10.1016/j.neuroimage.2019.03.079

Etchell, A. C., Civier, O., Ballard, K. J., \& Sowman, P. F. (2018). A systematic literature review of neuroimaging research on developmental stuttering between 1995 and 2016. Journal of Fluency Disorders, 55, 6-45. https://doi.org/10.1016/j.jfludis.2017.03.007

Etchell, A. C., Johnson, B. W., \& Sowman, P. F. (2014). Beta oscillations, timing, and stuttering. Frontiers in Human Neuroscience, 8, 1036. https://doi.org/10.3389/fnhum.2014.01036

Etchell, A. C., Ryan, M., Martin, E., Johnson, B. W., \& Sowman, P. F. (2016). Abnormal time course of low beta modulation in non-fluent preschool children: A magnetoencephalographic study of rhythm tracking. Neurolmage, 125, 953-963. https://doi.org/10.1016/j.neuroimage.2015.10.086

Euler, H. A., Gudenberg, A. W. v., Jung, K., \& Neumann, K. (2009). Computergestützte Therapie bei Redeflussstörungen: Die langfristige Wirksamkeit der Kasseler Stottertherapie (KST). Sprache · Stimme · Gehör, 33(04), 193-202. https://doi.org/10.1055/s-0029-1242747

Evers, E. A. T., Klaassen, E. B., Rombouts, S. A., Backes, W. H., \& Jolles, J. (2012). The effects of sustained cognitive task performance on subsequent resting state functional 


\section{References}

connectivity in healthy young and middle-aged male schoolteachers. Brain Connectivity, 2(2), 102-112. https://doi.org/10.1089/brain.2011.0060

Falk, S., Müller, T., \& Dalla Bella, S. (2015). Non-verbal sensorimotor timing deficits in children and adolescents who stutter. Frontiers in Psychology, 6, 847. https://doi.org/10.3389/fpsyg.2015.00847

Ferree, T. C., Luu, P., Russel, G. S., \& Tucker, D. M. (2001). Scalp electrode impedance, infection risk, and EEG data quality. Clinical Neurophysiology(112), Article 3, 536-544.

Field, A., Miles, J., \& Field, Z. (2013). Discovering statistics using R (Reprint). SAGE.

Fischer, T., Langner, R., Diers, K., Brocke, B., \& Birbaumer, N. (2010). Temporo-spatial dynamics of event-related EEG beta activity during the initial contingent negative variation. PloS One, 5(9). https://doi.org/10.1371/journal.pone.0012514

Floegel, M., Fuchs, S., \& Kell, C. A. (2020). Differential contributions of the two cerebral hemispheres to temporal and spectral speech feedback control. Nature Communications, 11(1), 2839. https://doi.org/10.1038/s41467-020-16743-2

Foundas, A. L., Mock, J. R., Corey, D. M., Golob, E. J., \& Conture, E. G. (2013). The SpeechEasy device in stuttering and nonstuttering adults: Fluency effects while speaking and reading. Brain and Language, 126(2), 141-150.

https://doi.org/10.1016/j.bandl.2013.04.004

Fox, J., \& Weisberg, S. (2019). An \{R\} Companion to Applied Regression (Version 3.0-6) [Computer software]. SAGE. Thousand Oaks, CA. https://socialsciences.mcmaster.ca/jfox/Books/Companion/

Frigerio-Domingues, C., \& Drayna, D. (2017). Genetic contributions to stuttering: The current evidence. Molecular Genetics \& Genomic Medicine, 5(2), 95-102. https://doi.org/10.1002/mgg3.276

Frigerio-Domingues, C. E., Gkalitsiou, Z., Zezinka, A., Sainz, E., Gutierrez, J., Byrd, C., Webster, R., \& Drayna, D. (2019). Genetic factors and therapy outcomes in persistent 


\section{References}

developmental stuttering. Journal of Communication Disorders, 80, 11-17. https://doi.org/10.1016/j.jcomdis.2019.03.007

Fujioka, T., Trainor, L. J., Large, E. W., \& Ross, B. (2012). Internalized timing of isochronous sounds is represented in neuromagnetic $\beta$ oscillations. The Journal of Neuroscience : The Official Journal of the Society for Neuroscience, 32(5), 1791-1802. https://doi.org/10.1523/JNEUROSCI.4107-11.2012

Garcia-Barrera, M. A., \& Davidow, J. H. (2015). Anticipation in stuttering: A theoretical model of the nature of stutter prediction. Journal of Fluency Disorders, 44, 1-15. https://doi.org/10.1016/j.jludis.2015.03.002

Garnett, E. O., Chow, H. M., Nieto-Castañón, A., Tourville, J. A., Guenther, F. H., \& Chang, S.-E. (2018). Anomalous morphology in left hemisphere motor and premotor cortex of children who stutter. Brain : A Journal of Neurology. Advance online publication. https://doi.org/10.1093/brain/awy199

Garyfallidis, E., Brett, M., Amirbekian, B., Rokem, A., van der Walt, S., Descoteaux, M., \& Nimmo-Smith, I. (2014). Dipy, a library for the analysis of diffusion MRI data. Frontiers in Neuroinformatics, 8, 8. https://doi.org/10.3389/fninf.2014.00008

Gehrig, J., Michalareas, G., Forster, M.-T., Lei, J., Hok, P., Laufs, H., Senft, C., Seifert, V., Schoffelen, J.-M., Hanslmayr, S., \& Kell, C. A. (2019). Low-Frequency Oscillations Code Speech during Verbal Working Memory. The Journal of Neuroscience : The Official Journal of the Society for Neuroscience, 39(33), 6498-6512. https://doi.org/10.1523/JNEUROSCI.0018-19.2019

Gehrig, J., Wibral, M., Arnold, C., \& Kell, C. A. (2012). Setting up the speech production network: How oscillations contribute to lateralized information routing. Frontiers in Psychology, 3, 169. https://doi.org/10.3389/fpsyg.2012.00169 


\section{References}

Gehringer, J. E., Arpin, D. J., Heinrichs-Graham, E., Wilson, T. W., \& Kurz, M. J. (2018). Neurophysiological changes in the visuomotor network after practicing a motor task. Journal of Neurophysiology, 120(1), 239-249. https://doi.org/10.1152/jn.00020.2018

Ghahremani, A., Wessel, J. R., Udupa, K., Neagu, B., Zhuang, P., Saha, U., Kalia, S. K., Hodaie, M., Lozano, A. M., Aron, A. R., \& Chen, R. (2018). Stopping and slowing manual and spoken responses: Similar oscillatory signatures recorded from the subthalamic nucleus. Brain and Language, 176, 1-10.

https://doi.org/10.1016/j.bandl.2017.10.009

Gilbertson, T., Lalo, E., Doyle, L., Di Lazzaro, V., Cioni, B., \& Brown, P. (2005). Existing motor state is favored at the expense of new movement during $13-35 \mathrm{~Hz}$ oscillatory synchrony in the human corticospinal system. The Journal of Neuroscience : The Official Journal of the Society for Neuroscience, 25(34), 7771-7779. https://doi.org/10.1523/JNEUROSCI.1762-05.2005

Giraud, A.-L., Neumann, K., Bachoud-Levi, A.-C., Gudenberg, A. W. von, Euler, H. A., Lanfermann, H., \& Preibisch, C. (2008). Severity of dysfluency correlates with basal ganglia activity in persistent developmental stuttering. Brain and Language, 104(2), 190-199. https://doi.org/10.1016/j.bandl.2007.04.005

Goto, M., Abe, O., Miyati, T., Yamasue, H., Gomi, T., \& Takeda, T. (2016). Head Motion and Correction Methods in Resting-state Functional MRI. Magnetic Resonance in Medical Sciences : MRMS : An Official Journal of Japan Society of Magnetic Resonance in Medicine, 15(2), 178-186. https://doi.org/10.2463/mrms.rev.2015-0060

Grabner, R. H., Brunner, C., Leeb, R., Neuper, C., \& Pfurtscheller, G. (2007). Eventrelated EEG theta and alpha band oscillatory responses during language translation. Brain Research Bulletin, 72(1), 57-65. https://doi.org/10.1016/j.brainresbull.2007.01.001 


\section{References}

Greenlee, J. D. W., Oya, H., Kawasaki, H., Volkov, I. O., Kaufman, O. P., Kovach, C., Howard, M. A., \& Brugge, J. F. (2004). A functional connection between inferior frontal gyrus and orofacial motor cortex in human. Journal of Neurophysiology, 92(2), 11531164. https://doi.org/10.1152/jn.00609.2003

Griffin, Z. (2003). A reversed word length effect in coordinating the preparation and articulation of words in speaking. Psychonomic Bulletin \& Review(10), Article 3, 603609.

Guenther, F. H. (2016). Neural control of speech. MIT Press.

Guenther, F. H., Ghosh, S. S., \& Tourville, J. A. (2006). Neural modeling and imaging of the cortical interactions underlying syllable production. Brain and Language(96(3)), 208-301.

Guenther, F. H., \& Vladusich, T. (2012). A Neural Theory of Speech Acquisition and Production. Journal of Neurolinguistics, 25(5), 408-422.

https://doi.org/10.1016/j.jneuroling.2009.08.006

Hagmann, P., Cammoun, L., Gigandet, X., Meuli, R., Honey, C. J., van Wedeen, J., \& Sporns, O. (2008). Mapping the structural core of human cerebral cortex (Vol. 6). https://doi.org/10.1371/journal.pbio.0060159

Hanslmayr, S., Aslan, A., Staudigl, T., Klimesch, W., Herrmann, C. S., \& Bäuml, K.-H. (2007). Prestimulus oscillations predict visual perception performance between and within subjects. Neurolmage, 37(4), 1465-1473. https://doi.org/10.1016/j.neuroimage.2007.07.011

Hartmann, T., Schlee, W., \& Weisz, N. (2012). It's only in your head: Expectancy of aversive auditory stimulation modulates stimulus-induced auditory cortical alpha desynchronization. Neurolmage, 60(1), 170-178. https://doi.org/10.1016/j.neuroimage.2011.12.034 


\section{References}

Hartsuiker, R. J., Kolk, H. H. J., \& Lickley, R. J. (2005). Stuttering on function words and content words: A computational test of the covert repair hypothesis. Phonological Encoding and Monitoring in Normal and Pathological Speech, 261-280.

Herman, A. B., Houde, J. F., Vinogradov, S., \& Nagarajan, S. S. (2013). Parsing the phonological loop: Activation timing in the dorsal speech stream determines accuracy in speech reproduction. The Journal of Neuroscience : The Official Journal of the Society for Neuroscience, 33(13), 5439-5453. https://doi.org/10.1523/JNEUROSCI.1472-12.2013

Hickok, G. (2012). Computational neuroanatomy of speech production. Nature Reviews. Neuroscience, 13(2), 135-145. https://doi.org/10.1038/nrn3158

Hickok, G., Houde, J., \& Rong, F. (2011). Sensorimotor integration in speech processing: Computational basis and neural organization. Neuron, 69(3), 407-422. https://doi.org/10.1016/j.neuron.2011.01.019

Hilger, A. I., Zelaznik, H., \& Smith, A. (2016). Evidence That Bimanual Motor Timing Performance Is Not a Significant Factor in Developmental Stuttering. Journal of Speech, Language, and Hearing Research : JSLHR, 59(4), 674-685. https://doi.org/10.1044/2016_JSLHR-S-15-0172

Hofmann, M. J., Stenneken, P., Conrad, M., \& Jacobs, A. M. (2007). Sublexical frequency measures for orthographic and phonological units in German. Behavior Research Methods, 39(3), 620-629. https://doi.org/10.3758/BF03193034

Honey, C. J., Sporns, O., Cammoun, L., Gigandet, X., Thiran, J. P., Meuli, R., \& Hagmann, P. (2009). Predicting human resting-state functional connectivity from structural connectivity. Proceedings of the National Academy of Sciences of the United States of America, 106(6), 2035-2040. https://doi.org/10.1073/pnas.0811168106

Howell, P. (2004). Assessment of Some Contemporary Theories of Stuttering That Apply to Spontaneous Speech. Contemporary Issues in Communication Science and 


\section{References}

Disorders(31(Spring)), 123-140.

https://pubs.asha.org/doi/pdf/10.1044/cicsd_31_S_123

Howell, P., \& Au-Yeung, J. (2007). Phonetic complexity and stuttering in Spanish. Clinical Linguistics \& Phonetics, 21(2), 111-127.

Howell, P., Au-Yeung, J., \& Sackin, S. (1999). Exchange of stuttering from function words to content words with age. Journal of Speech Language and Hearing Research, 42(2), 345-354. https://doi.org/10.1044/jslhr.4202.345

Howell, P., Au-Yeung, J., Yaruss, J. S., \& Eldridge, K. (2006). Phonetic difficulty and stuttering in English. Clinical Linguistics \& Phonetics, 20(9), 703-716. https://doi.org/10.1080/02699200500390990

Hubbard, C. P., \& Prins, D. (1994). Word familiarity, syllabic stress pattern, and stuttering. Journal of Speech, Language, and Hearing Research, 37(3), 564-571.

Huber, E., Donnelly, P. M., Rokem, A., \& Yeatman, J. D. (2018). Rapid and widespread white matter plasticity during an intensive reading intervention. Nature Communications, 9(1), 2260. https://doi.org/10.1038/s41467-018-04627-5

Jackson, E. S., Gracco, V., \& Zebrowski, P. M. (2020). Eliciting Stuttering in Laboratory Contexts. Journal of Speech, Language, and Hearing Research : JSLHR, 63(1), 143150. https://doi.org/10.1044/2019_JSLHR-S-19-0173

Jahfari, S., Waldorp, L., van den Wildenberg, W. P. M., Scholte, H. S., Ridderinkhof, K. R., \& Forstmann, B. U. (2011). Effective connectivity reveals important roles for both the hyperdirect (fronto-subthalamic) and the indirect (fronto-striatal-pallidal) fronto-basal ganglia pathways during response inhibition. The Journal of Neuroscience : The Official Journal of the Society for Neuroscience, 31(18), 6891-6899.

https://doi.org/10.1523/JNEUROSCI.5253-10.2011

Jbabdi, S., Sotiropoulos, S. N., Savio, A. M., Graña, M., \& Behrens, T. E. J. (2012). Model-based analysis of multishell diffusion MR data for tractography: How to get over 


\section{References}

fitting problems. Magnetic Resonance in Medicine, 68(6), 1846-1855.

https://doi.org/10.1002/mrm.24204

Jenkinson, M., Beckmann, C. F., Behrens, T. E. J., Woolrich, M. W., \& Smith, S. M. (2012). Fsl. Neurolmage, 62(2), 782-790.

https://doi.org/10.1016/j.neuroimage.2011.09.015

Jenkinson, N., \& Brown, P. (2011). New insights into the relationship between dopamine, beta oscillations and motor function. Trends in Neurosciences, 34(12), 611-618. https://doi.org/10.1016/j.tins.2011.09.003

Jensen, O., Goel, P., Kopell, N., Pohja, M., Hari, R., \& Ermentrout, B. (2005). On the human sensorimotor-cortex beta rhythm: Sources and modeling. Neurolmage, 26(2), 347-355. https://doi.org/10.1016/j.neuroimage.2005.02.008

Jensen, O., \& Mazaheri, A. (2010). Shaping functional architecture by oscillatory alpha activity: Gating by inhibition. Frontiers in Human Neuroscience, 4, 186.

https://doi.org/10.3389/fnhum.2010.00186

Jenson, D., Bowers, A. L., Harkrider, A. W., Thornton, D., Cuellar, M., \& Saltuklaroglu, T. (2014). Temporal dynamics of sensorimotor integration in speech perception and production: Independent component analysis of EEG data. Frontiers in Psychology, 5, 656. https://doi.org/10.3389/fpsyg.2014.00656

Jenson, D., Bowers, A. L., Hudock, D., \& Saltuklaroglu, T. (2019). The Application of EEG Mu Rhythm Measures to Neurophysiological Research in Stuttering. Frontiers in Human Neuroscience, 13, 458. https://doi.org/10.3389/fnhum.2019.00458 Jenson, D., Harkrider, A. W., Thornton, D., Bowers, A. L., \& Saltuklaroglu, T. (2015). Auditory cortical deactivation during speech production and following speech perception: An EEG investigation of the temporal dynamics of the auditory alpha rhythm. Frontiers in Human Neuroscience, 9, 534.

https://doi.org/10.3389/fnhum.2015.00534 


\section{References}

Jenson, D., Reilly, K. J., Harkrider, A. W., Thornton, D., \& Saltuklaroglu, T. (2018). Trait related sensorimotor deficits in people who stutter: An EEG investigation of $\mu$ rhythm dynamics during spontaneous fluency. Neurolmage. Clinical, 19, 690-702. https://doi.org/10.1016/j.nicl.2018.05.026

Joos, K., Ridder, D. de, Boey, R. A., \& Vanneste, S. (2014). Functional connectivity changes in adults with developmental stuttering: A preliminary study using quantitative electro-encephalography. Frontiers in Human Neuroscience, 8, 783.

https://doi.org/10.3389/fnhum.2014.00783

Jung, T.-P., Makeig, S., Humphries, C., Lee, T.-W., McKeown, M. J., Iragui, V., \& Sejnowski, T. J. (2000). Removing electroencephalographic artifacts by blind source separation. Psychophysiology(37), 163-178.

https://apps.dtic.mil/dtic/tr/fulltext/u2/a455940.pdf

Kadi-Hanifi, K., \& Howell, P. (1992). Syntactic analysis of the spontaneous speech of normally fluent and stuttering children. Journal of Fluency Disorders, 17(3), 151-170.

Kalveram, K. T. (2001). Neurobiology of speaking and stuttering. In H.-G. Bosshardt, J. S. Yaruss, \& H.F.M. Peters (Eds.), Fluency Disorders:: Theory, Research, Treatment and Self-help (pp. 59-65). Nijmegen University Press.

Kearney, E., \& Guenther, F. H. (2019). Articulating: The Neural Mechanisms of Speech Production. Language, Cognition and Neuroscience, 34(9), 1214-1229. https://doi.org/10.1080/23273798.2019.1589541

Kell, C. A., Neumann, K., Behrens, M., Gudenberg, A. W. von, \& Giraud, A.-L. (2018). Speaking-related changes in cortical functional connectivity associated with assisted and spontaneous recovery from developmental stuttering. Journal of Fluency Disorders(55), 135-144. 


\section{References}

Kell, C. A., Neumann, K., Kriegstein, K. von, Posenenske, C., Gudenberg, A. W. von, Euler, H., \& Giraud, A.-L. (2009). How the brain repairs stuttering. Brain : A Journal of Neurology, 132(Pt 10), 2747-2760. https://doi.org/10.1093/brain/awp185

Kell, C. A., Morillon, B., Kouneiher, F., \& Giraud, A.-L. (2011). Lateralization of speech production starts in sensory cortices--a possible sensory origin of cerebral left dominance for speech. Cerebral Cortex, 21(4), 932-937. https://doi.org/10.1093/cercor/bhq167

Keller, C., \& Kell, C. A. (2016). Asymmetric intra- and interhemispheric interactions during covert and overt sentence reading. Neuropsychologia, 93(Pt B), 448-465. https://doi.org/10.1016/j.neuropsychologia.2016.04.002

Kemerdere, R., Champfleur, N. M. de, Deverdun, J., Cochereau, J., Moritz-Gasser, S., Herbet, G., \& Duffau, H. (2016). Role of the left frontal aslant tract in stuttering: A brain stimulation and tractographic study. Journal of Neurology, 263(1), 157-167. https://doi.org/10.1007/s00415-015-7949-3

Kerlin, J. R., Shahin, A. J., \& Miller, L. M. (2010). Attentional gain control of ongoing cortical speech representations in a "cocktail party". The Journal of Neuroscience : The Official Journal of the Society for Neuroscience, 30(2), 620-628.

https://doi.org/10.1523/JNEUROSCI.3631-09.2010

Keuken, M. C., \& Forstmann, B. U. (2015). A probabilistic atlas of the basal ganglia using 7 T MRI. Data in Brief, 4, 577-582. https://doi.org/10.1016/j.dib.2015.07.028

Kilavik, B. E., Zaepffel, M., Brovelli, A., MacKay, W. A., \& Riehle, A. (2013). The ups and downs of $\beta$ oscillations in sensorimotor cortex. Experimental Neurology, 245, 15-26. https://doi.org/10.1016/j.expneurol.2012.09.014

Kim, K. S., Daliri, A., Flanagan, J. R., \& Max, L. (2020). Dissociated Development of Speech and Limb Sensorimotor Learning in Stuttering: Speech Auditory-motor 


\section{References}

Learning is Impaired in Both Children and Adults Who Stutter. Neuroscience, 451, 121. https://doi.org/10.1016/j.neuroscience.2020.10.014

Klimesch, W. (2012). A-band oscillations, attention, and controlled access to stored information. Trends in Cognitive Sciences, 16(12), 606-617. https://doi.org/10.1016/j.tics.2012.10.007

Klimesch, W., Sauseng, P., \& HansImayr, S. (2007). Eeg alpha oscillations: The inhibitiontiming hypothesis. Brain Research Reviews, 53(1), 63-88.

https://doi.org/10.1016/j.brainresrev.2006.06.003

Klostermann, F., Nikulin, V. V., Kühn, A. A., Marzinzik, F., Wahl, M., Pogosyan, A., Kupsch, A., Schneider, G.-H., Brown, P., \& Curio, G. (2007). Task-related differential dynamics of EEG alpha- and beta-band synchronization in cortico-basal motor structures. The European Journal of Neuroscience, 25(5), 1604-1615. https://doi.org/10.1111/j.1460-9568.2007.05417.x

Korzeczek, A., Primassin, A., Wolff von Gudenberg, A., Dechent, P., Paulus, W., Sommer, M., \& Neef, N. E. (2020). Fluent speech: neural basis of sensorimotor plasticity in developmental stuttering. BioRxiv. Advance online publication. https://doi.org/10.1101/2020.07.27.219360

Koshiyama, D., Fukunaga, M., Okada, N., Morita, K., Nemoto, K., Yamashita, F., Yamamori, H., Yasuda, Y., Matsumoto, J., Fujimoto, M., Kudo, N., Azechi, H., Watanabe, Y., Kasai, K., \& Hashimoto, R. (2020). Association between the superior longitudinal fasciculus and perceptual organization and working memory: A diffusion tensor imaging study. Neuroscience Letters, 738, 135349. https://doi.org/10.1016/j.neulet.2020.135349

Kotz, S. A., \& Schwartze, M. (2010). Cortical speech processing unplugged: A timely subcortico-cortical framework. Trends in Cognitive Sciences, 14(9), 392-399. https://doi.org/10.1016/j.tics.2010.06.005 


\section{References}

Kraft, S. J., Lowther, E., \& Beilby, J. (2019). The Role of Effortful Control in Stuttering Severity in Children: Replication Study. American Journal of Speech-Language Pathology, 28(1), 14-28. https://doi.org/10.1044/2018_AJSLP-17-0097

Kraft, S. J., \& Yairi, E. (2012). Genetic bases of stuttering: The state of the art, 2011. Folia Phoniatrica Et Logopaedica : Official Organ of the International Association of Logopedics and Phoniatrics (IALP), 64(1), 34-47. https://doi.org/10.1159/000331073

Kristofer E. Bouchard, Nima Mesgarani, Keith Johnson, \& Edward F. Chang. Functional organization of human sensorimotor cortex for speech articulation. https://www.nature.com/articles/nature11911.pdf?origin=ppub

Krogsrud, S. K., Fjell, A. M., Tamnes, C. K., Grydeland, H., Due-Tønnessen, P., Bjørnerud, A., Sampaio-Baptista, C., Andersson, J., Johansen-Berg, H., \& Walhovd, K. B. (2018). Development of white matter microstructure in relation to verbal and visuospatial working memory-A longitudinal study. PloS One, 13(4), e0195540. https://doi.org/10.1371/journal.pone.0195540

Kronfeld-Duenias, V., Amir, O., Ezrati-Vinacour, R., Civier, O., \& Ben-Shachar, M. (2016a). Dorsal and ventral language pathways in persistent developmental stuttering. Cortex; a Journal Devoted to the Study of the Nervous System and Behavior, 81, 7992. https://doi.org/10.1016/j.cortex.2016.04.001

Kronfeld-Duenias, V., Amir, O., Ezrati-Vinacour, R., Civier, O., \& Ben-Shachar, M. (2016b). The frontal aslant tract underlies speech fluency in persistent developmental stuttering. Brain Structure \& Function, 221(1), 365-381. https://doi.org/10.1007/s00429-014-0912-8

Kuhlen, A. K., Bogler, C., Brennan, S. E., \& Haynes, J.-D. (2017). Brains in dialogue: Decoding neural preparation of speaking to a conversational partner. Social Cognitive and Affective Neuroscience, 12(6), 871-880. https://doi.org/10.1093/scan/nsx018 


\section{References}

Laganaro, M. (2019). Phonetic encoding in utterance production: a review of open issues from 1989 to 2018. Language, Cognition and Neuroscience, 34(9), 1193-1201. https://doi.org/10.1080/23273798.2019.1599128

Laganaro, M., \& Alario, F.-X. (2006). On the locus of the syllable frequency effect in speech production. Journal of Memory and Language, 55(2), 178-196. https://doi.org/10.1016/j.jml.2006.05.001

Lauritzen, M., Mathiesen, C., Schaefer, K., \& Thomsen, K. J. (2012). Neuronal inhibition and excitation, and the dichotomic control of brain hemodynamic and oxygen responses. Neurolmage, 62(2), 1040-1050.

https://doi.org/10.1016/j.neuroimage.2012.01.040

Lehtelä, L., Salmelin, R., \& Hari, R. (1997). Evidence for reactive magnetic 10-Hz rhythm in the human auditory cortex. Neuroscience Letters(222), Article 2, 111-114.

Levelt, W. J. M. (2002). Picture naming and word frequency: Comments on Alario, Costa and Caramazza, Language and Cognitive Processes, 17(3), 299-319. Language and Cognitive Processes, 17(6), 663-671. https://doi.org/10.1080/01690960143000443

Levelt, W. J. M., Roelofs, A., \& Meyer, A. S. (1999). A theory of lexical access in speech production. Behavioral and Brain Sciences, 22(01).

https://doi.org/10.1017/S0140525X99001776

Levelt, W. J. M., \& Wheeldon, L. (1994). Do speakers have access to a mental syllabary? Cognition, 50(1-3), 239-269. https://doi.org/10.1016/0010-0277(94)90030-2

Liljeström, M., Kujala, J., Stevenson, C., \& Salmelin, R. (2015). Dynamic reconfiguration of the language network preceding onset of speech in picture naming. Human Brain Mapping, 36(3), 1202-1216. https://doi.org/10.1002/hbm.22697

Logan, K. J., \& Conture, E. G. (1995). Length, grammatical complexity, and rate differences in stuttered and fluent conversational utterances of children who stutter. Journal of Fluency Disorders, 20(1), 35-61. 


\section{References}

Logan, K. J., \& Conture, E. G. (1997). Selected temporal, grammatical, and phonological characteristics of conversational utterances produced by children who stutter. Journal of Speech, Language, and Hearing Research, 40(1), 107-120.

Long, M. A., Katlowitz, K. A., Svirsky, M. A., Clary, R. C., Byun, T. M., Majaj, N., Oya, H., Howard, M. A., \& Greenlee, J. D. W. (2016). Functional Segregation of Cortical Regions Underlying Speech Timing and Articulation. Neuron, 89(6), 1187-1193. https://doi.org/10.1016/j.neuron.2016.01.032

Loucks, T., Chon, H., \& Han, W. (2012). Audiovocal integration in adults who stutter. International Journal of Language \& Communication Disorders, 47(4), 451-456. https://doi.org/10.1111/j.1460-6984.2011.00111.x

Lu, C., Chen, C., Ning, N., Ding, G., Guo, T., Peng, D., Yang, Y., Li, K., \& Lin, C. (2010). The neural substrates for atypical planning and execution of word production in stuttering. Experimental Neurology, 221(1), 146-156. https://doi.org/10.1016/j.expneurol.2009.10.016

Lu, C., Chen, C., Peng, D., You, W., Zhang, X., Ding, G., Deng, X., Yan, Q., \& Howell, P. (2012). Neural anomaly and reorganization in speakers who stutter: A short-term intervention study. Neurology, 79(7), 625-632.

https://doi.org/10.1212/WNL.0b013e31826356d2

Lu, C., Peng, D., Chen, C., Ning, N., Ding, G., Li, K., Yang, Y., \& Lin, C. (2010). Altered effective connectivity and anomalous anatomy in the basal ganglia-thalamocortical circuit of stuttering speakers. Cortex; a Journal Devoted to the Study of the Nervous System and Behavior, 46(1), 49-67. https://doi.org/10.1016/j.cortex.2009.02.017

Lu, C., Zheng, L., Long, Y., Yan, Q., Ding, G., Liu, L., Peng, D., \& Howell, P. (2017). Reorganization of brain function after a short-term behavioral intervention for stuttering. Brain and Language, 168, 12-22. https://doi.org/10.1016/j.bandl.2017.01.001 


\section{References}

Maguire, G., Franklin, D., Vatakis, N. G., Morgenshtern, E., Denko, T., Yaruss, J. S., Spotts, C., Davis, L., Davis, A., Fox, P., Soni, P., Blomgren, M., Silverman, A., \& Riley, G. (2010). Exploratory randomized clinical study of pagoclone in persistent developmental stuttering: The EXamining Pagoclone for peRsistent dEvelopmental Stuttering Study. Journal of Clinical Psychopharmacology, 30(1), 48-56. https://doi.org/10.1097/JCP.0b013e3181caebbe

Maguire, G. A., Nguyen, D. L., Simonson, K. C., \& Kurz, T. L. (2020). The Pharmacologic Treatment of Stuttering and Its Neuropharmacologic Basis. Frontiers in Neuroscience, 14, 158. https://doi.org/10.3389/fnins.2020.00158

Maguire, G. A., Riley, G. D., Franklin, D. L., Maguire, M. E., Nguyen, C. T., \& Brojeni, P. H. (2004). Olanzapine in the treatment of developmental stuttering: A double-blind, placebo-controlled trial. Annals of Clinical Psychiatry : Official Journal of the American Academy of Clinical Psychiatrists, 16(2), 63-67. https://doi.org/10.1080/10401230490452834

Makris, N., Kennedy, D. N., Mclnerney, S., Sorensen, A. G., Wang, R., Caviness, V. S., \& Pandya, D. N. (2005). Segmentation of subcomponents within the superior longitudinal fascicle in humans: A quantitative, in vivo, DT-MRI study. Cerebral Cortex (New York, N.Y. : 1991), 15(6), 854-869. https://doi.org/10.1093/cercor/bhh186

Månsson, H. (2000). Childhood stuttering: Incidence and development. Journal of Fluency Disorders, 25(1), 47-57. https://doi.org/10.1016/S0094-730X(99)00023-6

Markett, S., Bleek, B., Reuter, M., Prüss, H., Richardt, K., Müller, T., Yaruss, J. S., \& Montag, C. (2016). Impaired motor inhibition in adults who stutter - evidence from speech-free stop-signal reaction time tasks. Neuropsychologia, 91, 444-450. https://doi.org/10.1016/j.neuropsychologia.2016.09.008 


\section{References}

Marreiros, A. C., Cagnan, H., Moran, R. J., Friston, K. J., \& Brown, P. (2013). Basal ganglia-cortical interactions in Parkinsonian patients. Neurolmage, 66, 301-310. https://doi.org/10.1016/j.neuroimage.2012.10.088

Max, L. (2004). Stuttering and internal models for sensorimotor control: A theoretical perspective to generate testable hypotheses. In B. Maassen, R. D. Kent, H.F.M. Peters, P. H.H.M. van Lieshout, \& W. Hulstijn (Eds.), Speech motor control in normal and disordered speech (1st ed., pp. 357-387). Oxford University Press.

Max, L., \& Daliri, A. (2019). Limited Pre-Speech Auditory Modulation in Individuals Who Stutter: Data and Hypotheses. Journal of Speech, Language, and Hearing Research : JSLHR, 62(8S), 3071-3084. https://doi.org/10.1044/2019_JSLHR-S-CSMC7-18-0358

Max, L., Daniels, J. C., Curet, K. M., \& Cronin, K. L. (2008). Modulation of auditory and somatosensory processing during planning of speech movements. In Proceedings of the 8th International Seminar on Speech Production (pp. 41-44).

Max, L., Guenther, F. H., Gracco, V. L., Ghosh, S. S., \& Wallace, M. E. (2004). Unstable or Insufficiently Activated Internal Models and Feedback-Biased Motor Control as Sources of Dysfluency:: A Theoretical Model of Stuttering. Contemporary Issues in Communication Science and Disorders(31), 105-122.

Max, L., Kadri, M., Mitsuya, T., \& Balasubramanian, V. (2019). Similar within-utterance loci of dysfluency in acquired neurogenic and persistent developmental stuttering. Brain and Language, 189, 1-9. https://doi.org/10.1016/j.bandl.2018.12.003

Max, L., \& Yudman, E. M. (2003). Accuracy and Variability of Isochronous Rhythmic Timing Across Motor Systems in Stuttering Versus Nonstuttering Individuals. Journal of Speech, Language, and Hearing Research : JSLHR, 46(1), 146-163.

https://doi.org/10.1044/1092-4388(2003/012)

McArdle, J. J., Mari, Z., Pursley, R. H., Schulz, G. M., \& Braun, A. R. (2009).

Electrophysiological evidence of functional integration between the language and motor 


\section{References}

systems in the brain: A study of the speech Bereitschaftspotential. Clinical Neurophysiology : Official Journal of the International Federation of Clinical Neurophysiology, 120(2), 275-284. https://doi.org/10.1016/j.clinph.2008.10.159

Melnick, K. S., \& Conture, E. G. (2000). Relationship of length and grammatical complexity to the systematic and nonsystematic speech errors and stuttering of children who stutter. Journal of Fluency Disorders(25), 21-45.

Menzies, R., O'Brian, S., Lowe, R., Packman, A., \& Onslow, M. (2016). International Phase II clinical trial of CBTPsych: A standalone Internet social anxiety treatment for adults who stutter. Journal of Fluency Disorders, 48, 35-43. https://doi.org/10.1016/j.jfludis.2016.06.002

Mersov, A., Cheyne, D., Jobst, C., \& Nil, L. de (2017). A preliminary study on the neural oscillatory characteristics of motor preparation prior to dysfluent and fluent utterances in adults who stutter. Journal of Fluency Disorders. Advance online publication. https://doi.org/10.1016/j.jludis.2017.05.003

Mersov, A.-M., Jobst, C., Cheyne, D. O., \& Nil, L. de (2016). Sensorimotor Oscillations Prior to Speech Onset Reflect Altered Motor Networks in Adults Who Stutter. Frontiers in Human Neuroscience, 10, 443. https://doi.org/10.3389/fnhum.2016.00443

Metzger, F. L., Auer, T., Helms, G., Paulus, W., Frahm, J., Sommer, M., \& Neef, N. E. (2018). Shifted dynamic interactions between subcortical nuclei and inferior frontal gyri during response preparation in persistent developmental stuttering. Brain Structure \& Function, 223(1), 165-182. https://doi.org/10.1007/s00429-017-1476-1

Meyer, A. S., Belke, E., Häcker, C., \& Mortensen Linda (2007). Use of word length information in utterance planning 弥. Journal of Memory and Language, 57(2), 210-231. https://doi.org/10.1016/j.jml.2006.10.005

Meyer, A. S., Roelofs, A., \& Levelt, W. J. M. (2003). Word length effects in object naming: The role of a response criterion. Journal of Memory and Language(48), 131-147. 


\section{References}

Mock, J. R., Foundas, A. L., \& Golob, E. J. (2015). Speech preparation in adults with persistent developmental stuttering. Brain and Language, 149, 97-105. https://doi.org/10.1016/j.bandl.2015.05.009

Mock, J. R., Foundas, A. L., \& Golob, E. J. (2016). Cortical activity during cued picture naming predicts individual differences in stuttering frequency. Clinical Neurophysiology : Official Journal of the International Federation of Clinical Neurophysiology, 127(9), 3093-3101. https://doi.org/10.1016/j.clinph.2016.06.005

Moll, K., \& Landerl, K. (Eds.). (2010). SLRT - II: Lese- und Rechtschreibtest: Weiterentwicklung des Salzburger Lese- und Rechtschreibtests (SLRT) (2. korrigierte Auflage). Huber.

Müller, N., \& Weisz, N. (2012). Lateralized auditory cortical alpha band activity and interregional connectivity pattern reflect anticipation of target sounds. Cerebral Cortex, 22(7), 1604-1613. https://doi.org/10.1093/cercor/bhr232

Namasivayam, A. K., \& van Lieshout, P. (2008). Investigating speech motor practice and learning in people who stutter. Journal of Fluency Disorders, 33(1), 32-51. https://doi.org/10.1016/j.jfludis.2007.11.005

Namasivayam, A. K., \& van Lieshout, P. (2011). Speech motor skill and stuttering. Journal of Motor Behavior, 43(6), 477-489. https://doi.org/10.1080/00222895.2011.628347

Namasivayam, A. K., van Lieshout, P., Mcllroy, W. E., \& Nil, L. de (2009). Sensory feedback dependence hypothesis in persons who stutter. Human Movement Science, 28(6), 688-707. https://doi.org/10.1016/j.humov.2009.04.004

Natke, U., \& Kohmäscher, A. (2020). Stottern. Springer Berlin Heidelberg. https://doi.org/10.1007/978-3-662-60942-2

Natke, U., Sandrieser, P., Pietrowsky, R., \& Kalveram, K. T. (2004). Stuttering and syllabic stress in preschool children.: Preliminary observations. In B. Maassen, R. D. Kent, 
6. References

H.F.M. Peters, P. H.H.M. van Lieshout, \& W. Hulstijn (Eds.), Speech motor control in normal and disordered speech (1st ed., pp. 258-261). Oxford University Press.

Neef, N. E., Paulus, W., Neef, A., Gudenberg, A. W. von, \& Sommer, M. (2011). Reduced intracortical inhibition and facilitation in the primary motor tongue representation of adults who stutter. Clinical Neurophysiology : Official Journal of the International Federation of Clinical Neurophysiology, 122(9), 1802-1811.

https://doi.org/10.1016/j.clinph.2011.02.003

Neef, N. E., Anwander, A., Bütfering, C., Schmidt-Samoa, C., Friederici, A. D., Paulus, W., \& Sommer, M. (2018). Structural connectivity of right frontal hyperactive areas scales with stuttering severity. Brain : A Journal of Neurology, 141(1), 191-204. https://doi.org/10.1093/brain/awx316

Neef, N. E., Anwander, A., \& Friederici, A. D. (2015). The Neurobiological Grounding of Persistent Stuttering: From Structure to Function. Current Neurology and Neuroscience Reports, 15(9), 63. https://doi.org/10.1007/s11910-015-0579-4

Neef, N. E., Bütfering, C., Anwander, A., Friederici, A. D., Paulus, W., \& Sommer, M. (2016). Left posterior-dorsal area 44 couples with parietal areas to promote speech fluency, while right area 44 activity promotes the stopping of motor responses. Neurolmage, 142, 628-644. https://doi.org/10.1016/j.neuroimage.2016.08.030

Neumann, K., Euler, H. A., Bosshardt, H.-G., Cook, S., Sandrieser, P., \& Sommer, M. (2017). The Pathogenesis, Assessment and Treatment of Speech Fluency Disorders. Deutsches Arzteblatt International, 114(22-23), 383-390. https://doi.org/10.3238/arztebl.2017.0383

Neumann, K., Euler, H. A., Bosshardt, H.-G., Cook, S., Sandrieser, P., Sommer, M., Thum, G., Euler, H. A., \& Schneider, P. (2017). Pathogenese, Diagnostik und Behandlung von Redeflussstörungen // Stottern und Poltern: Entstehung, Diagnose, Behandlung: Evidenz-und konsensbasierte interdisziplinäre S3-Leitlinie, AWMF- 


\section{References}

Registernummer 049-013 // Die Leitlinie zu Redeflussstörungen (1st ed.).

Sprachentwicklung: Band 9. PL Academic Research.

https://www.awmf.org/uploads/tx_szleitlinien/049-013I_S3_Redeflusstoerungen_201609-verlaengert.pdf https://doi.org/10.3726/b11133

Neumann, K., Euler, H. A., Gudenberg, A. W. von, Giraud, A.-L., Lanfermann, H., Gall, V., \& Preibisch, C. (2003). The nature and treatment of stuttering as revealed by fMRI. Journal of Fluency Disorders, 28(4), 381-410.

https://doi.org/10.1016/j.jfludis.2003.07.003

Neumann, K., Euler, H. A., Kob, M., Wolff von Gudenberg, A., Giraud, A.-L., Weissgerber, T., \& Kell, C. A. (2018). Assisted and unassisted recession of functional anomalies associated with dysprosody in adults who stutter. Journal of Fluency Disorders, 55, 120-134. https://doi.org/10.1016/j.jfludis.2017.09.003

Neumann, K., Preibisch, C., Euler, H. A., Gudenberg, A. W. von, Lanfermann, H., Gall, V., \& Giraud, A.-L. (2005). Cortical plasticity associated with stuttering therapy. Journal of Fluency Disorders, 30(1), 23-39. https://doi.org/10.1016/j.jfludis.2004.12.002

Newman, R. S., \& Bernstein Ratner, N. (2007). The Role of Selected Lexical Factors on Confrontation Naming Accuracy, Speed, and Fluency in Adults Who Do and Do Not Stutter. Journal of Speech, Language, and Hearing Research : JSLHR, 50(1), 196213. https://doi.org/10.1044/1092-4388(2007/016)

Niccolai, V., van Dijk, H., Franzkowiak, S., Finis, J., Südmeyer, M., Jonas, M., Thomalla, G., Siebner, H. R., Müller-Vahl, K., Münchau, A., Schnitzler, A., \& Biermann-Ruben, K. (2016). Increased beta rhythm as an indicator of inhibitory mechanisms in tourette syndrome. Movement Disorders : Official Journal of the Movement Disorder Society, 31(3), 384-392. https://doi.org/10.1002/mds.26454

Nickels, L., \& Howard, D. (2004). Dissociating Effects of Number of Phonemes, Number of Syllables, and Syllabic Complexity on Word Production in Aphasia: It's the Number of 


\section{References}

Phonemes that Counts. Cognitive Neuropsychology, 21(1), 57-78.

https://doi.org/10.1080/02643290342000122

Niziolek, C. A., \& Guenther, F. H. (2013). Vowel category boundaries enhance cortical and behavioral responses to speech feedback alterations. The Journal of Neuroscience : The Official Journal of the Society for Neuroscience, 33(29), 12090-12098. https://doi.org/10.1523/JNEUROSCI.1008-13.2013

Nye, C., Vanryckeghem, M., Schwartz, J. B., Herder, C., Turner, H. M., \& Howard, C. (2013). Behavioral Stuttering Interventions for Children and Adolescents: A Systematic Review and Meta-Analysis. Journal of Speech Language and Hearing Research, 56(3), 921. https://doi.org/10.1044/1092-4388(2012/12-0036)

Obleser, J., \& Weisz, N. (2012). Suppressed alpha oscillations predict intelligibility of speech and its acoustic details. Cerebral Cortex, 22(11), 2466-2477. https://doi.org/10.1093/cercor/bhr325

Olander, L., Smith, A., \& Zelaznik, H. N. (2010). Evidence That a Motor Timing Deficit Is a Factor in the Development of Stuttering. Journal of Speech, Language, and Hearing Research, 53(4), 876-886. https://doi.org/10.1044/1092-4388(2009/09-0007)

Oldfield, R. C. (1971). The assessment and analysis of handedness: The Edinburgh inventory. Neuropsychologia(9), 97-113.

http://andersgade.dk/Readings/Oldfield1971.pdf

Olthoff, A., Baudewig, J., Kruse, E., \& Dechent, P. (2008). Cortical sensorimotor control in vocalization: A functional magnetic resonance imaging study. The Laryngoscope, 118(11), 2091-2096. https://doi.org/10.1097/MLG.0b013e31817fd40f

Oostenveld, R., Fries, P., Maris, E., \& Schoffelen, J.-M. (2011). Fieldtrip: Open source software for advanced analysis of MEG, EEG, and invasive electrophysiological data. Computational Intelligence and Neuroscience, 2011, 156869. https://doi.org/10.1155/2011/156869 


\section{References}

Packman, A. (2012). Theory and therapy in stuttering: A complex relationship. Journal of Fluency Disorders, 37(4), 225-233. https://doi.org/10.1016/j.jludis.2012.05.004

Packman, A., Code, C., \& Onslow, M. (2007). On the cause of stuttering: Integrating theory with brain and behavioral research. Journal of Neurolinguistics, 20(5), 353-362. https://doi.org/10.1016/j.jneuroling.2006.11.001

Palen, C., \& Peterson, J. M. (1982). Word frequency and children's stuttering: The relationship to sentence structure. Journal of Fluency Disorders, 7(1), 55-62.

Palmer, C. E., Auksztulewicz, R., Ondobaka, S., \& Kilner, J. M. (2019). Sensorimotor beta power reflects the precision-weighting afforded to sensory prediction errors. Neurolmage, 200, 59-71. https://doi.org/10.1016/j.neuroimage.2019.06.034

Papitto, G., Friederici, A. D., \& Zaccarella, E. (2020). The topographical organization of motor processing: An ALE meta-analysis on six action domains and the relevance of Broca's region. Neurolmage, 206, 116321. https://doi.org/10.1016/j.neuroimage.2019.116321

Pastötter, B., Berchtold, F., \& Bäuml, K.-H. T. (2012). Oscillatory correlates of controlled speed-accuracy tradeoff in a response-conflict task. Human Brain Mapping, 33(8), 1834-1849. https://doi.org/10.1002/hbm.21322

Peirce, J. W. (2009). Generating Stimuli for Neuroscience Using PsychoPy. Frontiers in Neuroinformatics, 2, 10. https://doi.org/10.3389/neuro.11.010.2008

Peirce, J. W., \& MacAskill, M. (2018). Building experiments in PsychoPy. SAGE.

Perrin, F., Pernier, J., Bertrand, O., \& Echallier, J. F. (1989). Spherical splines for scalp potential and current density mapping. Electroencephalography and Clinical Neurophysiology(72), 184-187.

Petermann, F. (Ed.). (2012). Wechsler adult intelligence scale - fourth edition (WAIS-IV): Deutsche Bearbeitung. Pearson Assessment. 


\section{References}

Pfurtscheller, G., Neuper, C., Brunner, C., \& da Silva, F. L. (2005). Beta rebound after different types of motor imagery in man. Neuroscience Letters, 378(3), 156-159. https://doi.org/10.1016/j.neulet.2004.12.034

Pfurtscheller, G., Neuper, C., \& Mohl, W. (1994). Event-related desynchronization (ERD) during visual processing. International Journal of Psychophysiology : Official Journal of the International Organization of Psychophysiology(16), 147-153.

Piai, V., Roelofs, A., Rommers, J., Dahlslätt, K., \& Maris, E. (2015). Withholding planned speech is reflected in synchronized beta-band oscillations. Frontiers in Human Neuroscience, 9, 549. https://doi.org/10.3389/fnhum.2015.00549

Picazio, S., Veniero, D., Ponzo, V., Caltagirone, C., Gross, J., Thut, G., \& Koch, G. (2014). Prefrontal control over motor cortex cycles at beta frequency during movement inhibition. Current Biology : CB, 24(24), 2940-2945.

https://doi.org/10.1016/j.cub.2014.10.043

Piispala, J., Kallio, M., Bloigu, R., \& Jansson-Verkasalo, E. (2016). Delayed N2 response in Go condition in a visual Go/Nogo ERP study in children who stutter. Journal of Fluency Disorders, 48, 16-26. https://doi.org/10.1016/j.jludis.2016.02.001

Piispala, J., Starck, T., Jansson-Verkasalo, E., \& Kallio, M. (2018). Decreased occipital alpha oscillation in children who stutter during a visual Go/Nogo task. Clinical Neurophysiology : Official Journal of the International Federation of Clinical Neurophysiology, 129(9), 1971-1980. https://doi.org/10.1016/j.clinph.2018.06.022

Pollard, R., Ellis, J. B., Finan, D., \& Ramig, P. R. (2009). Effects of the SpeechEasy on Objective and Perceived Aspects of Stuttering: A 6-Month, Phase I Clinical Trial in Naturalistic Environments. Journal of Speech, Language, and Hearing Research : JSLHR, 52(2), 516-533. https://doi.org/10.1044/1092-4388(2008/07-0204) 


\section{References}

Pollok, B., Latz, D., Krause, V., Butz, M., \& Schnitzler, A. (2014). Changes of motorcortical oscillations associated with motor learning. Neuroscience, 275, 47-53. https://doi.org/10.1016/j.neuroscience.2014.06.008

Pollok, B., Boysen, A.-C., \& Krause, V. (2015). The effect of transcranial alternating current stimulation (tACS) at alpha and beta frequency on motor learning. Behavioural Brain Research, 293, 234-240. https://doi.org/10.1016/j.bbr.2015.07.049

Postma, A., \& Kolk, H. (1993). The Covert Repair Hypothesis. Journal of Speech, Language, and Hearing Research : JSLHR, 36(3), 472-487. https://doi.org/10.1044/jshr.3603.472

Power, J. D., Barnes, K. A., Snyder, A. Z., Schlaggar, B. L., \& Petersen, S. E. (2012). Spurious but systematic correlations in functional connectivity MRI networks arise from subject motion. Neurolmage, 59(3), 2142-2154. https://doi.org/10.1016/j.neuroimage.2011.10.018

Preibisch, C., Neumann, K., Raab, P., Euler, H. A., Gudenberg, A. W. von, Lanfermann, H., \& Giraud, A.-L. (2003). Evidence for compensation for stuttering by the right frontal operculum. Neurolmage, 20(2), 1356-1364. https://doi.org/10.1016/S1053$8119(03) 00376-8$

Price, C. J. (2010). The anatomy of language: A review of $100 \mathrm{fMRI}$ studies published in 2009. Annals of the New York Academy of Sciences, 1191, 62-88. https://doi.org/10.1111/j.1749-6632.2010.05444.x

Price, C. J. (2012). A review and synthesis of the first 20 years of PET and fMRI studies of heard speech, spoken language and reading. Neurolmage, 62(2), 816-847. https://doi.org/10.1016/j.neuroimage.2012.04.062

Primaßin, A. (2019). Longitudinal structural and functional brain changes associated with stuttering improvement by therapy or brain lesion [Dissertation]. Georg-August Universität, Göttingen. 


\section{References}

Qiao, J., Wang, Z., Zhao, G., Huo, Y., Herder, C. L., Sikora, C. O., \& Peterson, B. S. (2017). Functional neural circuits that underlie developmental stuttering. PloS One, 12(7), e0179255. https://doi.org/10.1371/journal.pone.0179255

R Core Team. (2019). R: A language and environment for statistical computing (Version 3.6.2) [Computer software]. R Foundation for Statistical. Vienna, Austria. https://www.R-project.org/

Rastatter, M. P., Stuart, A., \& Kalinowski, J. (1998). Quantitative electroencephalogram of posterior cortical areas of fluent and stuttering participants during reading with normal and altered auditory feedback. Perceptual and Motor Skills, 87(2), 623-633. https://doi.org/10.2466/pms.1998.87.2.623

Ratner, N. B., \& Sih, C. C. (1987). Effects of gradual increases in sentence length and complexity on children's dysfluency. Journal of Speech and Hearing Disorders, 52(3), 278-287.

Rauschecker, A. M., Pringle, A., \& Watkins, K. E. (2008). Changes in neural activity associated with learning to articulate novel auditory pseudowords by covert repetition. Human Brain Mapping, 29(11), 1231-1242. https://doi.org/10.1002/hbm.20460

Raza, M. H., Domingues, C. E. F., Webster, R., Sainz, E., Paris, E., Rahn, R., Gutierrez, J., Chow, H. M., Mundorff, J., Kang, C.-S., Riaz, N., Basra, M. A. R., Khan, S., Riazuddin, S., Moretti-Ferreira, D., Braun, A., \& Drayna, D. (2016). Mucolipidosis types II and III and non-syndromic stuttering are associated with different variants in the same genes. European Journal of Human Genetics : EJHG, 24(4), 529-534. https://doi.org/10.1038/ejhg.2015.154

Raza, M. H., Mattera, R., Morell, R., Sainz, E., Rahn, R., Gutierrez, J., Paris, E., Root, J., Solomon, B., Brewer, C., Basra, M. A. R., Khan, S., Riazuddin, S., Braun, A., Bonifacino, J. S., \& Drayna, D. (2015). Association between Rare Variants in AP4E1, a 


\section{References}

Component of Intracellular Trafficking, and Persistent Stuttering. American Journal of Human Genetics, 97(5), 715-725. https://doi.org/10.1016/j.ajhg.2015.10.007

Reilly, S., Onslow, M., Packman, A., Wake, M., Bavin, E. L., Prior, M., Eadie, P., Cini, E., Bolzonello, C., \& Ukoumunne, O. C. (2009). Predicting stuttering onset by the age of 3 years: A prospective, community cohort study. Pediatrics, 123(1), 270-277. https://doi.org/10.1542/peds.2007-3219

Richels, C., Buhr, A., Conture, E., \& Ntourou, K. (2010). Utterance complexity and stuttering on function words in preschool-age children who stutter. Journal of Fluency Disorders, 35(3), 314-331. https://doi.org/10.1016/j.jfludis.2010.06.001

Riecker, A., Brendel, B., Ziegler, W., Erb, M., \& Ackermann, H. (2008). The influence of syllable onset complexity and syllable frequency on speech motor control. Brain and Language, 107(2), 102-113. https://doi.org/10.1016/j.bandl.2008.01.008

Riley, G. (2009). The stuttering severity instrument for adults and children (SSI-4) (4th ed.). PRO-ED.

Riley, J., Riley, G., \& Maguire, G. (2004). Subjective Screening of Stuttering severity, locus of control and avoidance: Research edition. Journal of Fluency Disorders, 29(1), 51-62. https://doi.org/10.1016/j.jfludis.2003.12.001

Rödel, R. M. W., Olthoff, A., Tergau, F., Simonyan, K., Kraemer, D., Markus, H., \& Kruse, E. (2004). Human cortical motor representation of the larynx as assessed by transcranial magnetic stimulation (TMS). The Laryngoscope, 114(5), 918-922. https://doi.org/10.1097/00005537-200405000-00026

Romei, V., Gross, J., \& Thut, G. (2010). On the role of prestimulus alpha rhythms over occipito-parietal areas in visual input regulation: Correlation or causation? The Journal of Neuroscience : The Official Journal of the Society for Neuroscience, 30(25), 86928697. https://doi.org/10.1523/JNEUROSCI.0160-10.2010 


\section{References}

Rong, F., Isenberg, A. L., Sun, E., \& Hickok, G. (2018). The neuroanatomy of speech sequencing at the syllable level. PloS One, 13(10), e0196381. https://doi.org/10.1371/journal.pone.0196381

Ronson, I. (1976). Word frequency and stuttering: The relationship to sentence structure. Journal of Speech and Hearing Research, 19(4), 813-819.

Rosenfield, D. B. (2013). Neural anomaly and reorganization in speakers who stutter:: A short-term intervention study. Neurology, 80(16), 1538.

https://doi.org/10.1212/WNL.0b013e31828fa475

Sakai, K., \& Passingham, R. E. (2006). Prefrontal set activity predicts rule-specific neural processing during subsequent cognitive performance. The Journal of Neuroscience : The Official Journal of the Society for Neuroscience, 26(4), 1211-1218. https://doi.org/10.1523/JNEUROSCI.3887-05.2006

Saleh, M., Reimer, J., Penn, R., Ojakangas, C. L., \& Hatsopoulos, N. G. (2010). Fast and slow oscillations in human primary motor cortex predict oncoming behaviorally relevant cues. Neuron, 65(4), 461-471. https://doi.org/10.1016/j.neuron.2010.02.001

Salmelin, R., \& Sams, M. (2002). Motor cortex involvement during verbal versus nonverbal lip and tongue movements. Human Brain Mapping, 16(2), 81-91.

Salmelin, R., Schnitzler, A., Schmitz, F., \& Freund, H. (2000). Single word reading in developmental stutterers and fluent speakers. Brain(123), 184-202.

Saltuklaroglu, T., Harkrider, A. W., Thornton, D., Jenson, D., \& Kittilstved, T. (2017). Eeg $\mathrm{Mu}(\mu)$ rhythm spectra and oscillatory activity differentiate stuttering from non-stuttering adults. Neurolmage, 153, 232-245. https://doi.org/10.1016/j.neuroimage.2017.04.022

Sasisekaran, J., \& Weathers, E. J. (2019). Disfluencies and phonological revisions in a nonword repetition task in school-age children who stutter. Journal of Communication Disorders, 81, 105917. https://doi.org/10.1016/j.jcomdis.2019.105917 


\section{References}

Sasisekaran, J., \& Weisberg, S. (2014). Practice and retention of nonwords in adults who stutter. Journal of Fluency Disorders, 41, 55-71.

https://doi.org/10.1016/j.jfludis.2014.02.004

Saur, D., Kreher, B. W., Schnell, S., Kümmerer, D., Kellmeyer, P., Vry, M.-S., Umarova, R., Musso, M., Glauche, V., Abel, S., Huber, W., Rijntjes, M., Hennig, J., \& Weiller, C. (2008). Ventral and dorsal pathways for language. Proceedings of the National Academy of Sciences of the United States of America, 105(46), 18035-18040. https://doi.org/10.1073/pnas.0805234105

Schellig, D., \& Schächtele, B. (2002). Konsonanten-Trigramm-Test: (KTT). Swets Test Services $\mathrm{GmbH}$.

Schmidt, R., Herrojo Ruiz, M., Kilavik, B. E., Lundqvist, M., Starr, P. A., \& Aron, A. R. (2019). Beta Oscillations in Working Memory, Executive Control of Movement and Thought, and Sensorimotor Function. The Journal of Neuroscience : The Official Journal of the Society for Neuroscience, 39(42), 8231-8238.

https://doi.org/10.1523/JNEUROSCI.1163-19.2019

Schriefers, H., \& Teruel, E. (1999). Phonological Facilitation in the Production of Twoword Utterances. European Journal of Cognitive Psychology, 11(1), 17-50. https://doi.org/10.1080/713752301

Segawa, J. A., Tourville, J. A., Beal, D. S., \& Guenther, F. H. (2015). The neural correlates of speech motor sequence learning. Journal of Cognitive Neuroscience, 27(4), 819-831. https://doi.org/10.1162/jocn_a_00737

Sengupta, R., Shah, S., Gore, K., Loucks, T., \& Nasir, S. M. (2016). Anomaly in neural phase coherence accompanies reduced sensorimotor integration in adults who stutter. Neuropsychologia, 93(Pt A), 242-250.

https://doi.org/10.1016/j.neuropsychologia.2016.11.004 


\section{References}

Sengupta, R., Shah, S., Loucks, T. M. J., Pelczarski, K., Scott Yaruss, J., Gore, K., \& Nasir, S. M. (2017). Cortical dynamics of disfluency in adults who stutter. Physiological Reports, 5(9). https://doi.org/10.14814/phy2.13194

Sengupta, R., Yaruss, J. S., Loucks, T. M., Gracco, V. L., Pelczarski, K., \& Nasir, S. M. (2019). Theta Modulated Neural Phase Coherence Facilitates Speech Fluency in Adults Who Stutter. Frontiers in Human Neuroscience, 13, 394.

https://doi.org/10.3389/fnhum.2019.00394

Sheehan, J. G. (1974). Stuttering behavior: A phonetic analysis. Journal of Communication Disorders, 7(3), 193-212.

Sigurd, B., Eeg-Olofsson, M., \& van Weijer, J. (2004). Word length, sentence length and frequency - Zipf revisited. Studia Linguistica(58), Article 1, 37-52.

https://math.wvu.edu/ hdiamond/Math222F17/Sigurd_et_al-2004-

Studia_Linguistica.pdf

Siman-Tov, T., Bosak, N., Sprecher, E., Paz, R., Eran, A., Aharon-Peretz, J., \& Kahn, I. (2016). Early Age-Related Functional Connectivity Decline in High-Order Cognitive Networks. Frontiers in Aging Neuroscience, 8, 330.

https://doi.org/10.3389/fnagi.2016.00330

Simonyan, K., \& Fuertinger, S. (2015). Speech networks at rest and in action: Interactions between functional brain networks controlling speech production. Journal of Neurophysiology, 113(7), 2967-2978. https://doi.org/10.1152/jn.00964.2014

Simonyan, K., \& Horwitz, B. (2011). Laryngeal motor cortex and control of speech in humans. The Neuroscientist : A Review Journal Bringing Neurobiology, Neurology and Psychiatry, 17(2), 197-208. https://doi.org/10.1177/1073858410386727

Simonyan, K., Ostuni, J., Ludlow, C. L., \& Horwitz, B. (2009). Functional but not structural networks of the human laryngeal motor cortex show left hemispheric lateralization during syllable but not breathing production. The Journal of Neuroscience : The Official 
6. References

Journal of the Society for Neuroscience, 29(47), 14912-14923.

https://doi.org/10.1523/JNEUROSCI.4897-09.2009

Simpson, G. B., \& Kang, H. (2004). Syllable processing in alphabetic Korean. Reading and Writing, 17(1-2), 137-151.

Smith, A., Sadagopan, N., Walsh, B., \& Weber-Fox, C. (2010). Increasing phonological complexity reveals heightened instability in inter-articulatory coordination in adults who stutter. Journal of Fluency Disorders, 35(1), 1-18.

https://doi.org/10.1016/j.jfludis.2009.12.001

Smith, A., \& Weber, C. (2017). How Stuttering Develops: The Multifactorial Dynamic Pathways Theory. Journal of Speech, Language, and Hearing Research : JSLHR, 60(9), 2483-2505. https://doi.org/10.1044/2017_JSLHR-S-16-0343

Smits-Bandstra, S., De Nil, L., \& Rochon, E. (2006). The transition to increased automaticity during finger sequence learning in adult males who stutter. Journal of Fluency Disorders, 31(1), 22-42; quiz 39-40.

https://doi.org/10.1016/j.jfludis.2005.11.004

Smits-Bandstra, S., De Nil, L. F., \& Saint-Cyr, J. A. (2006). Speech and nonspeech sequence skill learning in adults who stutter. Journal of Fluency Disorders, 31(2), 116136. https://doi.org/10.1016/j.jfludis.2006.04.003

Sommer, M., Koch, M. A., Paulus, W., Weiller, C., \& Büchel, C. (2002). Disconnection of speech-relevant brain areas in persistent developmental stuttering. The Lancet, 360(9330), 380-383. https://doi.org/10.1016/S0140-6736(02)09610-1

Stancák, A., \& Pfurtscheller, G. (1996). Event-related desynchronisation of central betarhythms during brisk and slow self-paced finger movements of dominant and nondominant hand. Brain Research. Cognitive Brain Research, 4(3), 171-183. https://doi.org/10.1016/S0926-6410(96)00031-6 


\section{References}

Steele, V. R., Claus, E. D., Aharoni, E., Harenski, C., Calhoun, V. D., Pearlson, G., \& Kiehl, K. A. (2014). A large scale $(\mathrm{N}=102)$ functional neuroimaging study of error processing in a Go/NoGo task. Behavioural Brain Research, 268, 127-138. https://doi.org/10.1016/j.bbr.2014.04.001

Strauss, U., Grzybek, P., \& Altmann, G. (2006). Word Length and Word Frequency. In P. Gryzbek \& P. Grzybek (Eds.), Text, Speech and Language Technology: Vol. 31. Contributions to the Science of Text and Language: Word Length Studies and Related Issues (pp. 277-294). Springer.

Swann, N., Tandon, N., Canolty, R., Ellmore, T. M., McEvoy, L. K., Dreyer, S., DiSano, M., \& Aron, A. R. (2009). Intracranial EEG reveals a time- and frequency-specific role for the right inferior frontal gyrus and primary motor cortex in stopping initiated responses. The Journal of Neuroscience : The Official Journal of the Society for Neuroscience, 29(40), 12675-12685. https://doi.org/10.1523/JNEUROSCI.335909.2009

Swann, N. C., Cai, W., Conner, C. R., Pieters, T. A., Claffey, M. P., George, J. S., Aron, A. R., \& Tandon, N. (2012). Roles for the pre-supplementary motor area and the right inferior frontal gyrus in stopping action: Electrophysiological responses and functional and structural connectivity. Neurolmage, 59(3), 2860-2870.

https://doi.org/10.1016/j.neuroimage.2011.09.049

Tani, T., \& Sakai, Y. (2010). Stuttering after right cerebellar infarction: A case study. Journal of Fluency Disorders, 35(2), 141-145. https://doi.org/10.1016/j.jfludis.2010.03.001

Tani, T., \& Sakai, Y. (2011). Analysis of five cases with neurogenic stuttering following brain injury in the basal ganglia. Journal of Fluency Disorders, 36(1), 1-16. https://doi.org/10.1016/j.jfludis.2010.12.002 


\section{References}

Taylor, J. S. H., Rastle, K., \& Davis, M. H. (2013). Can cognitive models explain brain activation during word and pseudoword reading? A meta-analysis of 36 neuroimaging studies. Psychological Bulletin, 139(4), 766-791. https://doi.org/10.1037/a0030266

Tezel-Bayraktaroglu, O., Bayraktaroglu, Z., Demirtas-Tatlidede, A., Demiralp, T., \& Oge, A. E. (2020). Neuronavigated rTMS inhibition of right pars triangularis anterior in stuttering: Differential effects on reading and speaking. Brain and Language, 210, 104862. https://doi.org/10.1016/j.bandl.2020.104862

Theys, C., Nil, L. de, Thijs, V., van Wieringen, A., \& Sunaert, S. (2013). A crucial role for the cortico-striato-cortical loop in the pathogenesis of stroke-related neurogenic stuttering. Human Brain Mapping, 34(9), 2103-2112.

https://doi.org/10.1002/hbm.22052

Toft, M., \& Dietrichs, E. (2011). Aggravated stuttering following subthalamic deep brain stimulation in Parkinson's disease--two cases. BMC Neurology, 11, 44. https://doi.org/10.1186/1471-2377-11-44

Tomasi, D., \& Volkow, N. D. (2012). Aging and functional brain networks. Molecular Psychiatry, 17(5), 471, 549-58. https://doi.org/10.1038/mp.2011.81

Tourville, J. A., Reilly, K. J., \& Guenther, F. H. (2008). Neural mechanisms underlying auditory feedback control of speech. Neurolmage, 39(3), 1429-1443. https://doi.org/10.1016/j.neuroimage.2007.09.054

Toyomura, A., Fujii, T., \& Kuriki, S. (2015). Effect of an 8-week practice of externally triggered speech on basal ganglia activity of stuttering and fluent speakers. Neurolmage, 109, 458-468. https://doi.org/10.1016/j.neuroimage.2015.01.024

Treleaven, S. B., \& Coalson, G. A. (2020). Manual response inhibition and quality of life in adults who stutter. Journal of Communication Disorders, 88, 106053. https://doi.org/10.1016/j.jcomdis.2020.106053 


\section{References}

Tremblay, P., Deschamps, I., Baroni, M., \& Hasson, U. (2016). Neural sensitivity to syllable frequency and mutual information in speech perception and production. Neurolmage, 136, 106-121. https://doi.org/10.1016/j.neuroimage.2016.05.018

Turkeltaub, P. E., Eden, G. F., Jones, K. M., \& Zeffiro, T. A. (2002). Meta-analysis of the functional neuroanatomy of single-word reading: Method and validation. Neurolmage, 16(3 Pt 1), 765-780. https://doi.org/10.1006/nimg.2002.1131

Tzagarakis, C., Ince, N. F., Leuthold, A. C., \& Pellizzer, G. (2010). Beta-band activity during motor planning reflects response uncertainty. The Journal of Neuroscience : The Official Journal of the Society for Neuroscience, 30(34), 11270-11277. https://doi.org/10.1523/JNEUROSCI.6026-09.2010

Tzagarakis, C., West, S., \& Pellizzer, G. (2015). Brain oscillatory activity during motor preparation: Effect of directional uncertainty on beta, but not alpha, frequency band. Frontiers in Neuroscience, 9, 246. https://doi.org/10.3389/fnins.2015.00246

Vahdat, S., Darainy, M., Milner, T. E., \& Ostry, D. J. (2011). Functionally specific changes in resting-state sensorimotor networks after motor learning. The Journal of Neuroscience : The Official Journal of the Society for Neuroscience, 31(47), 1690716915. https://doi.org/10.1523/JNEUROSCI.2737-11.2011

van Dijk, H., Schoffelen, J.-M., Oostenveld, R., \& Jensen, O. (2008). Prestimulus oscillatory activity in the alpha band predicts visual discrimination ability. The Journal of Neuroscience : The Official Journal of the Society for Neuroscience, 28(8), 1816-1823. https://doi.org/10.1523/JNEUROSCI.1853-07.2008

van Lieshout, P. H.H.M., Hulstijn, W., \& Peters, H.F.M. (2004). Searching for the weak link in the speech production chain of people who stutter: A motor skill approach. In B. Maassen, R. D. Kent, H.F.M. Peters, P. H.H.M. van Lieshout, \& W. Hulstijn (Eds.), Speech motor control in normal and disordered speech (1st ed., pp. 314-345). Oxford University Press. 


\section{References}

Vanhoutte, S., Cosyns, M., van Mierlo, P., Batens, K., Corthals, P., Letter, M. de, van Borsel, J., Santens, P., Vanhoutte, S., Cosyns, M., van Mierlo, P., Batens, K., Corthals, P., Letter, M. de, van Borsel, J., \& Santens, P. (2016). When will a stuttering moment occur? The determining role of speech motor preparation. Neuropsychologia, 86, 93102. https://doi.org/10.1016/j.neuropsychologia.2016.04.018

Walden, T. A., Frankel, C. B., Buhr, A. P., Johnson, K. N., Conture, E. G., \& Karrass, J. M. (2012). Dual diathesis-stressor model of emotional and linguistic contributions to developmental stuttering. Journal of Abnormal Child Psychology, 40(4), 633-644. https://doi.org/10.1007/s10802-011-9581-8

Waldvogel, D., Gelderen van, P., Muellbacher, W., Ziemann, U., Immisch, I., \& Hallett, M. (2000). The relative metabolic demand on inhibition and exication. Nature(406), Article 31, 995-998.

Walker, H. C., Phillips, D. E., Boswell, D. B., Guthrie, B. L., Guthrie, S. L., Nicholas, A. P., Montgomery, E. B., \& Watts, R. L. (2009). Relief of Acquired Stuttering Associated With Parkinson's Disease by Unilateral Left Subthalamic Brain Stimulation. Journal of Speech, Language, and Hearing Research : JSLHR, 52(6), 1652-1657. https://doi.org/10.1044/1092-4388(2009/08-0089)

Warrington, S., Bryant, K. L., Khrapitchev, A. A., Sallet, J., Charquero-Ballester, M., Douaud, G., Jbabdi, S., Mars, R. B., \& Sotiropoulos, S. N. (2020). Xtract - Standardised protocols for automated tractography in the human and macaque brain. Neurolmage, 217, 116923. https://doi.org/10.1016/j.neuroimage.2020.116923

Watkins, K. E., Smith, S. M., Davis, S., \& Howell, P. (2008). Structural and functional abnormalities of the motor system in developmental stuttering. Brain : A Journal of Neurology, 131(Pt 1), 50-59. https://doi.org/10.1093/brain/awm241 


\section{References}

Weiss, S., \& Mueller, H. M. (2012). "Too Many betas do not Spoil the Broth": The Role of Beta Brain Oscillations in Language Processing. Frontiers in Psychology, 3, 201. https://doi.org/10.3389/fpsyg.2012.00201

Wessel, J. R., Ghahremani, A., Udupa, K., Saha, U., Kalia, S. K., Hodaie, M., Lozano, A. M., Aron, A. R., \& Chen, R. (2016). Stop-related subthalamic beta activity indexes global motor suppression in Parkinson's disease. Movement Disorders : Official Journal of the Movement Disorder Society, 31(12), 1846-1853.

https://doi.org/10.1002/mds.26732

Whitfield-Gabrieli, S., \& Nieto-Castanon, A. (2012). Conn: A functional connectivity toolbox for correlated and anticorrelated brain networks. Brain Connectivity, 2(3), 125141. https://doi.org/10.1089/brain.2012.0073

Wiecki, T. V., \& Frank, M. J. (2013). A computational model of inhibitory control in frontal cortex and basal ganglia. Psychological Review, 120(2), 329-355.

https://doi.org/10.1037/a0031542

Wilcox, R. (2019). WRS (Version 37) [Computer software]. USC Stevens Institute for Innovation, University of Southern California. Los Angeles, CA 90115, USA. https://www-rcf.usc.edu/ rwilcox/Rallfun-v37

Windsor, J., Kohnert, K., Lobitz, K. F., \& Pham, G. T. (2010). Cross-Language Nonword Repetition by Bilingual and Monolingual Children. American Journal of SpeechLanguage Pathology, 19(4), 298-310. https://doi.org/10.1044/1058-0360(2010/090064)

Wingate, M. E. (1964). A standard definition of stuttering. The Journal of Speech and Hearing Disorders, 29, 484-489. https://doi.org/10.1044/jshd.2904.484

Wingate, M. E. (1982). Early position and stuttering occurrence. Journal of Fluency Disorders(7), 243-285.

Wingate, M. E. (1988). The structure of stuttering: A psycholinguistic analysis. Springer. 


\section{References}

Wingate, M. E. (2002). Foundations of stuttering. Academic Press.

Woollams, A. M., Silani, G., Okada, K., Patterson, K., \& Price, C. J. (2011). Word or wordlike? Dissociating orthographic typicality from lexicality in the left occipito-temporal cortex. Journal of Cognitive Neuroscience, 23(4), 992-1002.

https://doi.org/10.1162/jocn.2010.21502

World Health Organization. (1998). Five Well Being Index. https://www.psykiatriregionh.dk/who-5/who-5-questionnaires/Pages/default.aspx

World Health Organization. (2011). International statistical classification of diseases and related health problems: The $I C D-10$ (10th revision, Fifth edition). World Health Organization. https://www.who.int/classifications/icd/en/bluebook.pdf

World Health Organization. (2019). International Classification of Diseases for Mortality and Morbidity Statistics: ICD-11. https://icd.who.int/icd11refguide/en/index.html

Wu, J. C., Maguire, G., Riley, G., Lee, A., Keator, D., Tang, C., Fallon, J., \& Najafi, A. (1997). Increased dopamine activity associated with stuttering. NeuroReport(8), 767770.

Xuan, Y., Meng, C., Yang, Y., Zhu, C., Wang, L., Yan, Q., Lin, C., \& Yu, C. (2012). Resting-State Brain activity in adult males who stutter. PloS One(7(1)), e39579. https://doi.org/10.1371/journal.pone.0030570.t001

Xue, G., Aron, A. R., \& Poldrack, R. A. (2008). Common neural substrates for inhibition of spoken and manual responses. Cerebral Cortex, 18(8), 1923-1932. https://doi.org/10.1093/cercor/bhm220

Yada, Y., Tomisato, S., \& Hashimoto, R.-I. (2019). Online cathodal transcranial direct current stimulation to the right homologue of Broca's area improves speech fluency in people who stutter. Psychiatry and Clinical Neurosciences, 73(2), 63-69. https://doi.org/10.1111/pcn.12796 


\section{References}

Yairi, E., \& Ambrose, N. (2013). Epidemiology of stuttering: 21st century advances. Journal of Fluency Disorders, 38(2), 66-87. https://doi.org/10.1016/j.jfludis.2012.11.002

Yaruss, J. S. (1999). Utterance length, syntactic complexity, and childhood stuttering. Journal of Speech, Language, and Hearing Research, 42(2), 329-344.

Yaruss, J. S., \& Conture, E. G. (1996). Stuttering and phonological disorders in children: Examination of the Covert Repair Hypothesis. Journal of Speech Language and Hearing Research, 39(2), 349-364. https://doi.org/10.1044/jshr.3902.349

Yaruss, J. S., \& Quesal, R. W. (2006). Overall Assessment of the Speaker's Experience of Stuttering (OASES): Documenting multiple outcomes in stuttering treatment. Journal of Fluency Disorders, 31(2), 90-115. https://doi.org/10.1016/j.jfludis.2006.02.002

Yaruss, J. S., \& Quesal, R. W. (2014). OASES: Overall Assessment of the Speaker's Experience of Stuttering. Pearson Assessment.

Yaruss, J. S., Quesal, R. W., \& Coleman, C. E. (Eds.). (2016). Overall assessment of the speaker's experience of stuttering: Erfassung der Erfahrung von stotternden Erwachsenen. Übersetzt ins Deutsche. Stuttering Therapy Resources, Inc.

Yeatman, J. D., Dougherty, R. F., Myall, N. J., Wandell, B. A., \& Feldman, H. M. (2012). Tract profiles of white matter properties: Automating fiber-tract quantification. PloS One, 7(11), e49790. https://doi.org/10.1371/journal.pone.0049790

Yilmaz, G., Ungan, P., Sebik, O., Uginčius, P., \& Türker, K. S. (2014). Interference of tonic muscle activity on the EEG: A single motor unit study. Frontiers in Human Neuroscience, 8, 504. https://doi.org/10.3389/fnhum.2014.00504

Zaepffel, M., Trachel, R., Kilavik, B. E., \& Brochier, T. (2013). Modulations of EEG beta power during planning and execution of grasping movements. PloS One, 8(3), e60060. https://doi.org/10.1371/journal.pone.0060060

Zavala, B. A., Jang, A. I., \& Zaghloul, K. A. (2017). Human subthalamic nucleus activity during non-motor decision making. ELife(6), 1-23. https://doi.org/10.7554/eLife.31007 
6. References

Zhang, R., Geng, X., \& Lee, T. M. C. (2017). Large-scale functional neural network correlates of response inhibition: An fMRI meta-analysis. Brain Structure \& Function, 222(9), 3973-3990. https://doi.org/10.1007/s00429-017-1443-x

Ziegler, W. (2009). Modelling the architecture of phonetic plans: Evidence from apraxia of speech. Language and Cognitive Processes, 24(5), 631-661.

https://doi.org/10.1080/01690960802327989 
Appendix A: Increased right frontal beta power during speech intention in severe stuttering

\section{Appendices}

Appendix A: Increased right frontal beta power during speech intention in severe stuttering

Table 1. Demographic information per participant.

\begin{tabular}{|c|c|c|c|c|c|c|c|c|c|}
\hline \multirow[b]{2}{*}{ ID } & \multirow[b]{2}{*}{ Group } & \multirow[b]{2}{*}{ Sex } & \multicolumn{2}{|l|}{ Age } & \multirow{2}{*}{$\begin{array}{l}\text { laterality } \\
\text { quotient, } \\
\text { rH }\end{array}$} & \multirow{2}{*}{$\begin{array}{l}\text { Stuttering } \\
\text { severity } \\
\text { (SSI-4) }\end{array}$} & \multirow{2}{*}{$\begin{array}{l}\text { SSI-4 } \\
\text { total } \\
\text { score }\end{array}$} & \multirow[b]{2}{*}{$\begin{array}{l}\text { SSI-4 } \\
R+S\end{array}$} & \multirow{2}{*}{$\begin{array}{l}\text { OASES } \\
\text { total } \\
\text { score }\end{array}$} \\
\hline & & & $\begin{array}{l}\text { in } \\
\text { years }\end{array}$ & Education & & & & & \\
\hline S01 & $A W S$ & $m$ & 35 & 3 & 78 & very mild & 15 & 3 & 1.9 \\
\hline SO2 & AWS & $m$ & 31 & 4 & 81 & very mild & 16 & 4 & 1.36 \\
\hline SO3 & $A W S$ & $m$ & 57 & 2 & 100 & mild & 18 & 6 & 1.88 \\
\hline SO4 & AWS & $m$ & 21 & 2 & 90 & mild & 24 & 8 & 1.2 \\
\hline S05 & AWS & $m$ & 22 & 2 & 89 & very severe & 38 & 16 & 2.47 \\
\hline So6 & AWS & $m$ & 31 & 2 & 60 & moderate & 30 & 14 & 1.55 \\
\hline SO7 & AWS & $m$ & 26 & 3 & 100 & mild & 19 & 5 & 1.78 \\
\hline S08 & AWS & $m$ & 20 & 2 & 100 & very mild & 17 & 6 & 1.77 \\
\hline Sog & $A W S$ & $m$ & 21 & 3 & 88 & very mild & 17 & 4 & 2.19 \\
\hline$S 10$ & $A W S$ & $m$ & 49 & 3 & 70 & very severe & 49 & 18 & 2.64 \\
\hline$S 11$ & AWS & $m$ & 31 & 4 & 100 & moderate & 26 & 11 & 2.29 \\
\hline S12 & AWS & $m$ & 28 & 3 & 89 & severe & 32 & 11 & 2.31 \\
\hline$S 13$ & AWS & $m$ & 19 & 3 & 88 & moderate & 28 & 9 & 1.29 \\
\hline S14 & AWS & $f$ & 21 & 3 & 60 & mild & 20 & 6 & 1.8 \\
\hline S15 & AWS & $m$ & 52 & 3 & 100 & very severe & 45 & 15 & 3.09 \\
\hline S16 & AWS & $m$ & 24 & 3 & 91 & moderate & 26 & 12 & 2.91 \\
\hline S17 & AWS & $f$ & 21 & 3 & 100 & very severe & 38 & 17 & 1.79 \\
\hline$S 18$ & AWS & $m$ & 23 & 3 & 100 & moderate & 25 & 9 & 2.62 \\
\hline S19 & AWS & $f$ & 19 & 1 & 88 & moderate & 25 & 11 & 2.37 \\
\hline S20 & $F C$ & $m$ & 22 & 3 & 100 & no stuttering & 2 & 0 & - \\
\hline$S 21$ & $F C$ & $f$ & 27 & 3 & 75 & no stuttering & 4 & 2 & - \\
\hline S22 & $F C$ & $m$ & 23 & 3 & 100 & no stuttering & 6 & 2 & - \\
\hline S23 & $F C$ & $m$ & 24 & 2 & 100 & no stuttering & 8 & 4 & - \\
\hline S24 & $F C$ & $m$ & 30 & 3 & 100 & no stuttering & 7 & 2 & - \\
\hline S25 & $F C$ & $m$ & 26 & 3 & 24 & no stuttering & 6 & 2 & - \\
\hline S26 & $F C$ & $m$ & 22 & 3 & 74 & no stuttering & 6 & 2 & - \\
\hline S27 & $F C$ & $f$ & 26 & 3 & 87 & no stuttering & 6 & 2 & - \\
\hline S28 & $F C$ & $m$ & 31 & 4 & 40 & no stuttering & 8 & 4 & - \\
\hline S29 & $F C$ & $m$ & 22 & 3 & 100 & no stuttering & 9 & 4 & - \\
\hline
\end{tabular}


Appendix A: Increased right frontal beta power during speech intention in severe stuttering

$\begin{array}{lllllllll}\text { S30 } & F C & m & 29 & 3 & 88 & \text { no stuttering } & 8 & 2 \\ \text { S31 } & F C & m & 22 & 3 & 67 & \text { no stuttering } & 2 & 0 \\ \text { S32 } & F C & m & 23 & 3 & 88 & \text { no stuttering } & 4 & 2 \\ \text { S33 } & F C & m & 21 & 3 & 100 & \text { no stuttering } & 6 & 2 \\ \text { S34 } & F C & m & 19 & 3 & 75 & \text { no stuttering } & 9 & 2 \\ \text { S35 } & F C & m & 24 & 3 & 89 & \text { no stuttering } & 6 & 4 \\ \text { S36 } & F C & f & 57 & 2 & 100 & \text { no stuttering } & 0 & 0 \\ \text { S37 } & F C & m & 31 & 4 & 30 & \text { no stuttering } & 0 & 0 \\ \text { S38 } & F C & m & 21 & 3 & 0 & \text { no stuttering } & 4 & 2\end{array}$

Note. Age: represented in years. SSI-4: Stuttering severity instrument (Riley, 2009). R + S: Sum of SSI scores for reading a text and for a spontaneous speech sample. OASES: Overall Assessment of the Speaker's Experience of Stuttering (Yaruss et al., 2016). AWS = adults with developmental stuttering. $\mathrm{FC}=$ fluent controls. $\mathrm{m}=$ male; $\mathrm{f}=$ female; Education: 1 = high school, $2=<2$ years college, $3=>2$ years college, $4=$ post graduate.

Table 2. Pre-assessment scores per participant

\begin{tabular}{llccccccc}
\hline ID & Group & $\begin{array}{l}\text { SLRT-II } \\
\text { words, } \mathbf{n}\end{array}$ & $\begin{array}{l}\text { SLRT-II } \\
\text { pseudo- } \\
\text { words, } \mathbf{n}\end{array}$ & $\begin{array}{l}\text { Konso- } \\
\text { nanten- } \\
\text { Trigram- } \\
\text { Test }\end{array}$ & $\begin{array}{l}\text { Digit } \\
\text { span, } \\
\text { forwards }\end{array}$ & $\begin{array}{l}\text { Digit span, } \\
\text { backwards }\end{array}$ & $\begin{array}{l}\text { BDI- } \\
\text { II }\end{array}$ & $\begin{array}{l}\text { Well } \\
\text { Being }\end{array}$ \\
\hline S01 & AWS & 115 & 73 & 53 & 10 & 12 & 4 & 18 \\
S02 & AWS & 107 & 59 & 35 & 8 & 8 & 6 & 17 \\
S03 & AWS & 94 & 47 & 47 & 7 & 5 & 5 & 6 \\
S04 & AWS & 94 & 68 & 39 & 8 & 8 & 0 & 22 \\
S05 & AWS & 64 & 48 & 32 & 9 & 8 & 18 & 9 \\
S06 & AWS & 85 & 45 & 45 & 10 & 9 & 2 & 21 \\
S07 & AWS & 129 & 71 & 41 & 11 & 7 & 0 & 18 \\
S08 & AWS & 111 & 75 & 46 & 9 & 7 & 0 & 21 \\
S09 & AWS & 114 & 76 & 44 & 8 & 8 & 3 & 15 \\
S10 & AWS & $-*$ & - & 60 & 10 & 9 & 0 & 19 \\
S11 & AWS & 61 & 52 & 45 & 12 & 7 & 0 & 20 \\
S12 & AWS & 118 & 55 & 59 & 10 & 9 & 2 & 18 \\
S13 & AWS & 134 & 72 & 52 & 12 & 11 & 1 & 18 \\
S14 & AWS & 107 & 60 & 31 & 8 & 8 & 2 & 17 \\
S15 & AWS & 97 & 46 & 59 & 8 & 8 & 0 & 11 \\
S16 & AWS & 106 & 64 & 40 & 10 & 9 & 5 & 15 \\
S17 & AWS & 104 & 76 & 41 & 13 & 10 & 3 & 21 \\
S18 & AWS & 136 & 92 & 47 & 10 & 10 & 1 & 20 \\
S19 & AWS & 109 & 72 & 55 & 11 & 9 & 7 & 15 \\
S20 & FC & 123 & 64 & 55 & 7 & 10 & 3 & 13
\end{tabular}


Appendix A: Increased right frontal beta power during speech intention in severe stuttering

$\begin{array}{lllllllll}S 21 & F C & 93 & 71 & 46 & 11 & 12 & 20 & 5 \\ S 22 & F C & 97 & 61 & 39 & 8 & 9 & 9 & 19 \\ S 23 & F C & 98 & 61 & 40 & 10 & 11 & 2 & 17 \\ S 24 & F C & 118 & 77 & 56 & 10 & 9 & 0 & 18 \\ S 25 & F C & 121 & 79 & 45 & 9 & 10 & 0 & 19 \\ S 26 & F C & 73 & 57 & 46 & 10 & 7 & 7 & 12 \\ S 27 & F C & 152 & 106 & 50 & 11 & 11 & 14 & 9 \\ S 28 & F C & 118 & 86 & 38 & 11 & 10 & 16 & 15 \\ S 29 & F C & 112 & 75 & 55 & 10 & 6 & 12 & 8 \\ S 30 & F C & 98 & 85 & 48 & 11 & 8 & 4 & 13 \\ S 31 & F C & 114 & 65 & 50 & 9 & 10 & 7 & 16 \\ S 32 & F C & 119 & 64 & 51 & 10 & 9 & 2 & 20 \\ S 33 & F C & 135 & 97 & 45 & 13 & 11 & 1 & 22 \\ S 34 & F C & 118 & 81 & 53 & 10 & 10 & 0 & 20 \\ S 35 & F C & 93 & 77 & 52 & 13 & 11 & 0 & 21 \\ S 36 & F C & 109 & 73 & 53 & 11 & 11 & 1 & 16 \\ S 37 & F C & 152 & 102 & 47 & 7 & 11 & 3 & 11 \\ S 38 & F C & 127 & 87 & 55 & 15 & 15 & 9 & 10\end{array}$

Note. If not indicated otherwise, numbers represent test scores. ${ }^{*}$ due to stuttering severity this test could not be assessed. SLRT-II = Salzburger-Lese-Rechtschreib Test (Moll \& Landerl, 2010), total number of read words/pseuowords in one minute. Konsonanten Trigram Test (Schellig \& Schächtele, 2002). Digit span tests (Petermann, 2012) .BDI = Beck's Depression inventory (Beck et al., 1996). Well-being test (World Health Organization, 1998). AWS = adults with developmental stuttering. $\mathrm{FC}=$ Fluent controls. 
7. Appendices

Appendix B: Effects of word length and syllable frequency in adults with developmental stuttering

Appendix B: Effects of word length and syllable frequency in adults with developmental stuttering

Table 1. Demographic information per participant.

\begin{tabular}{|c|c|c|c|c|c|c|c|c|c|}
\hline ID & Group & Sex & $\begin{array}{l}\text { Age } \\
\text { in } \\
\text { years }\end{array}$ & $\begin{array}{l}\text { Edu- } \\
\text { cation }\end{array}$ & $\begin{array}{l}\text { right } \\
\text { hand, } \\
\text { laterality } \\
\text { quotient }\end{array}$ & $\begin{array}{l}\text { Stuttering } \\
\text { severity } \\
\text { (SSI-4) }\end{array}$ & $\begin{array}{l}\text { SSI-4 } \\
\text { total } \\
\text { score }\end{array}$ & $\begin{array}{l}\text { SSI-4 } \\
R+S\end{array}$ & $\begin{array}{l}\text { OASES } \\
\text { total } \\
\text { score }\end{array}$ \\
\hline S01 & $A W S$ & $m$ & 35 & 3 & 78 & very mild & 15 & 3 & 1.9 \\
\hline S02 & $A W S$ & $m$ & 31 & 4 & 81 & very mild & 16 & 4 & 1.36 \\
\hline SO3 & $A W S$ & $m$ & 57 & 2 & 100 & mild & 18 & 6 & 1.88 \\
\hline SO4 & $A W S$ & $m$ & 21 & 2 & 90 & mild & 24 & 8 & 1.2 \\
\hline SO5 & $A W S$ & $m$ & 22 & 2 & 89 & very severe & 38 & 16 & 2.47 \\
\hline S06 & $A W S$ & $m$ & 31 & 2 & 60 & moderate & 30 & 14 & 1.55 \\
\hline S07 & $A W S$ & $m$ & 26 & 3 & 100 & mild & 19 & 5 & 1.78 \\
\hline S08 & AWS & $m$ & 20 & 2 & 100 & very mild & 17 & 6 & 1.77 \\
\hline So9 & $A W S$ & $m$ & 21 & 3 & 88 & very mild & 17 & 4 & 2.19 \\
\hline$S 10$ & $A W S$ & $f$ & 27 & 3 & 20 & severe & 33 & 15 & 3.11 \\
\hline$S 11$ & $A W S$ & $m$ & 31 & 4 & 100 & moderate & 26 & 11 & 2.29 \\
\hline$S 12$ & $A W S$ & $m$ & 28 & 3 & 89 & severe & 32 & 11 & 2.31 \\
\hline$S 13$ & AWS & $m$ & 19 & 3 & 88 & moderate & 28 & 9 & 1.29 \\
\hline$S 14$ & AWS & $f$ & 21 & 3 & 60 & mild & 20 & 6 & 1.8 \\
\hline$S 15$ & AWS & $m$ & 52 & 3 & 100 & very severe & 45 & 15 & 3.09 \\
\hline$S 16$ & $A W S$ & $m$ & 24 & 3 & 91 & moderate & 26 & 12 & 2.91 \\
\hline$S 17$ & AWS & $f$ & 21 & 3 & 100 & very severe & 38 & 17 & 1.79 \\
\hline$S 18$ & $A W S$ & $m$ & 23 & 3 & 100 & moderate & 25 & 9 & 2.62 \\
\hline$S 19$ & AWS & $f$ & 19 & 1 & 88 & moderate & 25 & 11 & 2.37 \\
\hline$S 20$ & $F C$ & $m$ & 22 & 3 & 100 & no stuttering & 2 & 0 & - \\
\hline$S 21$ & $F C$ & $f$ & 27 & 3 & 75 & no stuttering & 4 & 2 & - \\
\hline S22 & $F C$ & $m$ & 23 & 3 & 100 & no stuttering & 6 & 2 & - \\
\hline S23 & $F C$ & $m$ & 24 & 2 & 100 & no stuttering & 8 & 4 & - \\
\hline S24 & $F C$ & $m$ & 30 & 3 & 100 & no stuttering & 7 & 2 & - \\
\hline S25 & $F C$ & $m$ & 26 & 3 & 24 & no stuttering & 6 & 2 & - \\
\hline$S 26$ & $F C$ & $m$ & 22 & 3 & 74 & no stuttering & 6 & 2 & - \\
\hline S27 & $F C$ & $f$ & 26 & 3 & 87 & no stuttering & 6 & 2 & - \\
\hline$S 28$ & $F C$ & $m$ & 31 & 4 & 40 & no stuttering & 8 & 4 & - \\
\hline S29 & $F C$ & $m$ & 22 & 3 & 100 & no stuttering & 9 & 4 & - \\
\hline S30 & $F C$ & $m$ & 29 & 3 & 88 & no stuttering & 8 & 2 & - \\
\hline$S 31$ & $F C$ & $m$ & 22 & 3 & 67 & no stuttering & 2 & 0 & - \\
\hline
\end{tabular}


7. Appendices

Appendix B: Effects of word length and syllable frequency in adults with developmental stuttering

$\begin{array}{lllllllll}\text { S32 } & F C & m & 23 & 3 & 88 & \text { no stuttering } & 4 & 2 \\ \text { S33 } & F C & m & 21 & 3 & 100 & \text { no stuttering } & 6 & 2 \\ \text { S34 } & F C & m & 19 & 3 & 75 & \text { no stuttering } & 9 & 2 \\ \text { S35 } & F C & m & 24 & 3 & 89 & \text { no stuttering } & 6 & 4 \\ \text { S36 } & F C & f & 57 & 2 & 100 & \text { no stuttering } & 0 & 0 \\ \text { S37 } & F C & m & 31 & 4 & 30 & \text { no stuttering } & 0 & 0 \\ \text { S38 } & F C & m & 21 & 3 & 0 & \text { no stuttering } & 4 & 2\end{array}$

Note. Age: represented in years. SSI-4: Stuttering severity instrument (Riley, 2009). R + S: Sum of SSI scores for reading a text and for a spontaneous speech sample. OASES: Overall Assessment of the Speaker's Experience of Stuttering (Yaruss et al., 2016). AWS = adults with developmental stuttering. $\mathrm{FC}=$ fluent controls. $m=$ male; $f=$ female. Education: $1=$ high school, $2=<2$ years college, 3 = >2years college, 4 = post graduate.

Table 2. Pre-assessment scores per participant.

\begin{tabular}{|c|c|c|c|c|c|c|c|c|}
\hline ID & Group & $\begin{array}{l}\text { SLRT-II } \\
\text { words, n }\end{array}$ & $\begin{array}{l}\text { SLRT-II } \\
\text { pseudo- } \\
\text { words, n }\end{array}$ & $\begin{array}{l}\text { Konso- } \\
\text { nanten- } \\
\text { Trigram- } \\
\text { Test }\end{array}$ & $\begin{array}{l}\text { Digit } \\
\text { span, } \\
\text { forwards }\end{array}$ & $\begin{array}{l}\text { Digit span, } \\
\text { backwards }\end{array}$ & $\begin{array}{l}\text { BDI- } \\
\text { II }\end{array}$ & $\begin{array}{l}\text { Well } \\
\text { Being }\end{array}$ \\
\hline S01 & $A W S$ & 115 & 73 & 53 & 10 & 12 & 4 & 18 \\
\hline SO2 & AWS & 107 & 59 & 35 & 8 & 8 & 6 & 17 \\
\hline SO3 & $A W S$ & 94 & 47 & 47 & 7 & 5 & 5 & 6 \\
\hline SO4 & $A W S$ & 94 & 68 & 39 & 8 & 8 & 0 & 22 \\
\hline SO5 & AWS & 64 & 48 & 32 & 9 & 8 & 18 & 9 \\
\hline So6 & AWS & 85 & 45 & 45 & 10 & 9 & 2 & 21 \\
\hline S07 & $A W S$ & 129 & 71 & 41 & 11 & 7 & 0 & 18 \\
\hline 508 & AWS & 111 & 75 & 46 & 9 & 7 & 0 & 21 \\
\hline$S 09$ & AWS & 114 & 76 & 44 & 8 & 8 & 3 & 15 \\
\hline S10 & AWS & 92 & 74 & 55 & 11 & 10 & 8 & 18 \\
\hline$S 11$ & AWS & 61 & 52 & 45 & 12 & 7 & 0 & 20 \\
\hline$S 12$ & AWS & 118 & 55 & 59 & 10 & 9 & 2 & 18 \\
\hline$S 13$ & $A W S$ & 134 & 72 & 52 & 12 & 11 & 1 & 18 \\
\hline S14 & AWS & 107 & 60 & 31 & 8 & 8 & 2 & 17 \\
\hline$S 15$ & $A W S$ & 97 & 46 & 59 & 8 & 8 & 0 & 11 \\
\hline$S 16$ & $A W S$ & 106 & 64 & 40 & 10 & 9 & 5 & 15 \\
\hline S17 & AWS & 104 & 76 & 41 & 13 & 10 & 3 & 21 \\
\hline$S 18$ & AWS & 136 & 92 & 47 & 10 & 10 & 1 & 20 \\
\hline$S 19$ & $A W S$ & 109 & 72 & 55 & 11 & 9 & 7 & 15 \\
\hline S2O & $F C$ & 123 & 64 & 55 & 7 & 10 & 3 & 13 \\
\hline S21 & $F C$ & 93 & 71 & 46 & 11 & 12 & 20 & 5 \\
\hline S22 & $F C$ & 97 & 61 & 39 & 8 & 9 & 9 & 19 \\
\hline
\end{tabular}




\section{Appendices}

Appendix B: Effects of word length and syllable frequency in adults with developmental stuttering

$\begin{array}{lllllllll}S 23 & F C & 98 & 61 & 40 & 10 & 11 & 2 & 17 \\ S 24 & F C & 118 & 77 & 56 & 10 & 9 & 0 & 18 \\ S 25 & F C & 121 & 79 & 45 & 9 & 10 & 0 & 19 \\ S 26 & F C & 73 & 57 & 46 & 10 & 7 & 7 & 12 \\ S 27 & F C & 152 & 106 & 50 & 11 & 11 & 14 & 9 \\ S 28 & F C & 118 & 86 & 38 & 11 & 10 & 16 & 15 \\ S 29 & F C & 112 & 75 & 55 & 10 & 6 & 12 & 8 \\ S 30 & F C & 98 & 85 & 48 & 11 & 8 & 4 & 13 \\ S 31 & F C & 114 & 65 & 50 & 9 & 10 & 7 & 16 \\ S 32 & F C & 119 & 64 & 51 & 10 & 9 & 2 & 20 \\ S 33 & F C & 135 & 97 & 45 & 13 & 11 & 1 & 22 \\ S 34 & F C & 118 & 81 & 53 & 10 & 10 & 0 & 20 \\ S 35 & F C & 93 & 77 & 52 & 13 & 11 & 0 & 21 \\ S 36 & F C & 109 & 73 & 53 & 11 & 11 & 1 & 16 \\ S 37 & F C & 152 & 102 & 47 & 7 & 11 & 3 & 11 \\ S 38 & F C & 127 & 87 & 55 & 15 & 15 & 9 & 10\end{array}$

Note. If not indicated otherwise, numbers represent test scores. SLRT-II = Salzburger-LeseRechtschreib Test (Moll \& Landerl, 2010), total number of read words/pseuowords in one minute. Konsonanten Trigram Test (Schellig \& Schächtele, 2002). Digit span tests (Petermann, 2012). $\mathrm{BDI}=$ Beck's Depression inventory (Beck et al., 1996). Well-being test (World Health Organization, 1998). AWS $=$ adults with developmental stuttering. $F C=$ Fluent controls 
7. Appendices

Appendix B: Effects of word length and syllable frequency in adults with developmental stuttering

Table 3. List of pseudowords.

\begin{tabular}{|c|c|c|c|c|c|c|}
\hline \multirow{2}{*}{ Orthographic } & \multirow{2}{*}{ SAMPA } & \multirow{2}{*}{ Syllable structure } & \multicolumn{3}{|c|}{ Number of } & \multirow{2}{*}{$\begin{array}{l}\text { IPC } \\
\text { score }\end{array}$} \\
\hline & & & syl & let & pho & \\
\hline Pierisch & pi:-rIS & CV-CVC & 2 & 8 & 5 & 3 \\
\hline Pierischbe & pi:-rlS-be: & CV-CVC-CV & 3 & 10 & 7 & 5 \\
\hline Pierischbeol & pi:-rlS-be:-o:I & CV-CVC-CV-VC & 4 & 12 & 9 & 7 \\
\hline Peurisch & pOy-rIS & CV-CVC & 2 & 8 & 5 & 3 \\
\hline Peurischbe & pOy-rIS-be: & CV-CVC-CV & 3 & 10 & 7 & 5 \\
\hline Peurischbeol & pOy-rlS-be:-o:I & CV-CVC-CV-VC & 4 & 12 & 9 & 7 \\
\hline Weilauk & vai-lauk & CV-CVC & 2 & 7 & 5 & 4 \\
\hline Weilauknä & vai-lauk-nE & CV-CVC-CV & 3 & 9 & 7 & 6 \\
\hline Weilauknätaum & vai-lauk-nE-taum & CV-CVC-CV-CVC & 4 & 13 & 10 & 7 \\
\hline Weulauk & vOy-lauk & CV-CVC & 2 & 7 & 5 & 4 \\
\hline Weulauknä & vOy-lauk-nE & CV-CVC-CV & 3 & 9 & 7 & 6 \\
\hline Weulauknätaum & vOy-lauk-nE-taum & CV-CVC-CV-CVC & 4 & 13 & 10 & 7 \\
\hline Mähxo & mE:-kso: & CV-CCV & 2 & 6 & 5 & 4 \\
\hline Mähxokul & mE:-kso:-kUl & CV-CCV-CVC & 3 & 8 & 7 & 8 \\
\hline Mähxokulgan & mE:-kso:-kUl-gan & CV-CCV-CVC-CVC & 4 & 11 & 11 & 10 \\
\hline Mäxo & mE-kso: & CV-CCV & 2 & 5 & 5 & 4 \\
\hline Mäxokul & mE-kso:-kUl & CV-CCV-CVC & 3 & 7 & 7 & 8 \\
\hline Mäxokulgan & mE-kso:-kUl-gan & CV-CCV-CVC-CVC & 4 & 10 & 11 & 10 \\
\hline Möhzas & m\&:-tsas & CV-CCVC & 2 & 6 & 6 & 4 \\
\hline Möhzaske & m\&:-tsas-ke: & CV-CCVC-CV & 3 & 8 & 8 & 7 \\
\hline Möhzaskeof & m\&:-tsas-ke:-Of & CV-CCVC-CV-VC & 4 & 10 & 10 & 9 \\
\hline Müzas & mY-tsas & CV-CCVC & 2 & 5 & 6 & 4 \\
\hline Müzaske & mY-tsas-ke: & CV-CCVC-CV & 3 & 7 & 8 & 7 \\
\hline Müzaskeof & mY-tsas-ke:-Of & CV-CCVC-CV-VC & 4 & 9 & 10 & 9 \\
\hline Mühxas & my:-ksas & CV-CCVC & 2 & 6 & 6 & 6 \\
\hline Mühxasji & my:-ksas-ji: & CV-CCVC-CV & 3 & 8 & 8 & 8 \\
\hline Mühxasjime & my:-ksas-ji:-m@ & $\mathrm{CV}-\mathrm{CCV} C-\mathrm{CV}-\mathrm{CV}$ & 4 & 10 & 10 & 8 \\
\hline Meuxas & mOy-ksas & CV-CCVC & 2 & 6 & 6 & 6 \\
\hline Meuxasji & mOy-ksas-ji: & CV-CCVC-CV & 3 & 8 & 8 & 8 \\
\hline Meuxasjime & mOy-ksas-ji:-m@ & CV-CCVC-CV-CV & 4 & 10 & 10 & 8 \\
\hline Muhxisch & mu:-ksIS & CV-CCVC & 2 & 8 & 6 & 6 \\
\hline Muhxischta & mu:-ksIS-ta & CV-CCVC-CV & 3 & 10 & 8 & 7 \\
\hline
\end{tabular}


7. Appendices

Appendix B: Effects of word length and syllable frequency in adults with developmental stuttering

\begin{tabular}{|c|c|c|c|c|c|}
\hline Muhxischtageus & mu:-ksIS-ta-gOys & CV-CCVC-CV-CVC & 4 & 14 & 11 \\
\hline Maxisch & ma-ksIS & CV-ccVC & 2 & 7 & 6 \\
\hline Maxischta & ma-ksIS-ta & CV-CCVC-CV & 3 & 9 & 8 \\
\hline Maxischtageus & ma-ksIS-ta-gOys & CV-CCVC-CV-CVC & 4 & 13 & 11 \\
\hline Murche & mUr-xE: & CVC-CV & 2 & 6 & 5 \\
\hline Murchese & mUr-xE:-z@ & CVC-CV-CV & 3 & 8 & 7 \\
\hline Murcheseku & mUr-xE:-z@-ku: & CVC-CV-CV-CV & 4 & 10 & 9 \\
\hline Mürche & mYr-xE: & CVC-CV & 2 & 6 & 5 \\
\hline Mürchese & mYr-xE:-z@ & CVC-CV-CV & 3 & 8 & 7 \\
\hline Mürcheseku & mYr-xE:-z@-ku: & CVC-CV-CV-CV & 4 & 10 & 9 \\
\hline Möhkdei & m\&:k-dai & CVC-CV & 2 & 8 & 6 \\
\hline Möhkdeigo & m\&:k-dai-go: & CVC-CV-CV & 3 & 11 & 8 \\
\hline Möhkdeigotaus & m\&:k-dai-go:-taus & CVC-CV-CV-CVC & 4 & 13 & 10 \\
\hline Mähkdei & $m E: k-d a i$ & CVC-CV & 2 & 8 & 6 \\
\hline Mähkdeigo & mE:k-dai-go & CVC-CV-CV & 3 & 11 & 8 \\
\hline Mähkdeigotaus & mE:k-dai-go:-taus & CVC-CV-CV-CVC & 4 & 13 & 10 \\
\hline Fakti & fak-ti: & CVC-CV & 2 & 8 & 6 \\
\hline Faktinäsch & fak-nES-\|lx & CVC-CV-CVC & 3 & 12 & 9 \\
\hline Faktinäschlich & fak-nES-IIx-ti: & CVC-CV-CVC-CVC & 4 & 14 & 11 \\
\hline Fagti & fa:k-ti: & CVC-CV & 2 & 9 & 6 \\
\hline Fagtinäsch & fa:k-nES-IIx & CVC-CV-CVC & 3 & 13 & 9 \\
\hline Fagtinäschlich & fa:k-nES-IIx-ti: & CVC-CV-CVC-CVC & 4 & 15 & 11 \\
\hline Wikno & vlk-no: & CVC-CV & 2 & 5 & 5 \\
\hline Wiknoxe & vlk-no:-kse: & CVC-CV-CCV & 3 & 7 & 8 \\
\hline Wiknoxegäm & vlk-no:-kse:-gEm & CVC-CV-CCV-CVC & 4 & 10 & 11 \\
\hline Wukno & vUk-no: & CVC-CV & 2 & 5 & 5 \\
\hline Wuknoxe & vUk-no:-kse: & CVC-CV-CCV & 3 & 7 & 8 \\
\hline Wuknoxegäm & vUk-no:-kse:-gEm & CVC-CV-CCV-CVC & 4 & 10 & 11 \\
\hline Mienlei & mi:n-lai & CVC-CV & 2 & 7 & 5 \\
\hline Mienleigrot & mi:n-lai-grOt & CVC-CV-CCVC & 3 & 11 & 9 \\
\hline Mienleigrotne & mi:n-lai-grOt-n@ & CVC-CV-CCVC-CV & 4 & 13 & 11 \\
\hline Muhnlei & mu:n-lai & CVC-CV & 2 & 7 & 5 \\
\hline Muhnleigrot & mu:n-lai-grOt & CVC-CV-CCVC & 3 & 11 & 9 \\
\hline Muhnleigrotne & mu:n-lai-grOt-n@ & CVC-CV-CCVC-CV & 4 & 13 & 11 \\
\hline Masri & mas-rl & CVC-CV & 2 & 5 & 5 \\
\hline Masrizäng & mas-rl-tsEN & CVC-CV-CCVC & 3 & 9 & 9 \\
\hline
\end{tabular}


7. Appendices

Appendix B: Effects of word length and syllable frequency in adults with developmental stuttering

\begin{tabular}{|c|c|c|c|c|c|}
\hline Masrizänge & mas-rl-tsEN-N@ & CVC-CV-CCVC-CV & 4 & 10 & 11 \\
\hline Mehsri & me:s-rl & CVC-CV & 2 & 6 & 5 \\
\hline Mehsrizäng & me:s-rl-tsEN & CVC-CV-CCVC & 3 & 10 & 9 \\
\hline Mehsrizänge & me:s-rl-tsEN-N@ & CVC-CV-CCVC-CV & 4 & 11 & 11 \\
\hline Wochdap & vOx-dap & CVC-CVC & 2 & 7 & 6 \\
\hline Wochdapsö & vOx-dap-s\&: & CVC-CVC-CV & 3 & 9 & 8 \\
\hline Wochdapsöge & vOx-dap-s\&:-g@ & CVC-CVC-CV-CV & 4 & 11 & 10 \\
\hline Wiechdap & vi:x-dap & CVC-CVC & 2 & 8 & 6 \\
\hline Wiechdapsö & vi:x-dap-s\&: & CVC-CVC-CV & 3 & 10 & 8 \\
\hline Wiechdapsöge & vi:x-dap-s\&:-g@ & CVC-CVC-CV-CV & 4 & 12 & 10 \\
\hline Fesgack & fEs-gak & CVC-CVC & 2 & 7 & 6 \\
\hline Fesgackbo & fEs-gak-bo: & CVC-CVC-CV & 3 & 9 & 7 \\
\hline Fesgackbofe & fEs-gak-bo:-f@ & CVC-CVC-CV-CV & 4 & 11 & 10 \\
\hline Fösgack & f\&:s-gak & CVC-CVC & 2 & 8 & 6 \\
\hline Fösgackbo & f\&:s-gak-bo: & CVC-CVC-CV & 3 & 10 & 8 \\
\hline Fösgackbofe & f\&:s-gak-bo:-f@ & CVC-CVC-CV-CV & 4 & 12 & 10 \\
\hline Mängläf & mEN-IEf & CVC-CVC & 2 & 7 & 6 \\
\hline Mängläfchü & mEN-IEf-xy: & CVC-CVC-CV & 3 & 10 & 8 \\
\hline Mängläfchübi & mEN-IEf-xy:-bi: & CVC-CVC-CV-CV & 4 & 12 & 10 \\
\hline Mingläf & mIN-IEf & CVC-CVC & 2 & 7 & 6 \\
\hline Mingläfchü & mIN-IEf-xy: & CVC-CVC-CV & 3 & 10 & 8 \\
\hline Mingläfchübi & mIN-IEf-xy:-bi: & CVC-CVC-CV-CV & 4 & 12 & 10 \\
\hline Markir & mar-klr & CVC-CVC & 2 & 6 & 6 \\
\hline Markirtu & mar-klr-tu: & CVC-CVC-CV & 3 & 8 & 8 \\
\hline Markirtuka & mar-klr-tu:-ka: & CVC-CVC-CV-CV & 4 & 10 & 10 \\
\hline Mirkir & mlr-klr & CVC-CVC & 2 & 6 & 6 \\
\hline Mirkirtu & mlr-klr-tu: & CVC-CVC-CV & 3 & 8 & 8 \\
\hline Mirkirtuka & mlr-kIr-tu:-ka: & CVC-CVC-CV-CV & 4 & 10 & 10 \\
\hline Mamkun & mam-kUn & CVC-CVC & 2 & 6 & 6 \\
\hline Mamkunlo & mam-kUn-lo: & CVC-CVC-CV & 3 & 8 & 8 \\
\hline Mamkunlodü & mam-kUn-lo:-dy: & CVC-CVC-CV-CV & 4 & 10 & 10 \\
\hline Mahmkun & ma:m-kUn & CVC-CVC & 2 & 7 & 6 \\
\hline Mahmkunlo & ma:m-kUn-lo: & CVC-CVC-CV & 3 & 9 & 8 \\
\hline Mahmkunlodü & ma:m-kUn-lo:-dy: & CVC-CVC-CV-CV & 4 & 11 & 10 \\
\hline Bäslun & bEs-IUn & CVC-CVC & 2 & 6 & 6 \\
\hline Bäslundeu & bEs-IUn-dOy & CVC-CVC-CV & 3 & 9 & 8 \\
\hline
\end{tabular}


7. Appendices

Appendix B: Effects of word length and syllable frequency in adults with developmental stuttering

\begin{tabular}{|c|c|c|c|c|c|}
\hline Bäslundeurus & bEs-IUn-dOy-rUs & CVC-CVC-CV-CVC & 4 & 12 & 11 \\
\hline Bähslun & bE:s-IUn & CVC-CVC & 2 & 7 & 6 \\
\hline Bähslundeu & bE:s-IUn-dOy & CVC-CVC-CV & 3 & 10 & 8 \\
\hline Bähslundeurus & bE:s-IUn-dOy-rUs & CVC-CVC-CV-CVC & 4 & 13 & 11 \\
\hline Fänrusch & fEn-rUS & CVC-CVC & 2 & 8 & 6 \\
\hline Fänruschmä & fEn-rUS-mE & CVC-CVC-CV & 3 & 10 & 8 \\
\hline Fänruschmäsül & fEn-rUS-mE-zYI & CVC-CVC-CV-CVC & 4 & 13 & 11 \\
\hline Fehnrusch & fe:n-rUS & CVC-CVC & 2 & 9 & 6 \\
\hline Fehnruschmä & fe:n-rUS-mE & CVC-CVC-CV & 3 & 11 & 8 \\
\hline Fehnruschmäsül & fe:n-rUS-mE-zYI & CVC-CVC-CV-CVC & 4 & 14 & 11 \\
\hline Maksahm & mak-za:m & CVC-CVC & 2 & 7 & 6 \\
\hline Maksahmlu & mak-za:m-lu: & CVC-CVC-CV & 3 & 9 & 8 \\
\hline Maksahmlutak & mak-za:m-lu:-tak & CVC-CVC-CV-CVC & 4 & 12 & 11 \\
\hline Mäksahm & mEk-za:m & CVC-CVC & 2 & 7 & 6 \\
\hline Mäksahmlu & mEk-za:m-lu: & CVC-CVC-CV & 3 & 9 & 8 \\
\hline Mäksahmlutak & mEk-za:m-lu:-tak & CVC-CVC-CV-CVC & 4 & 12 & 11 \\
\hline Maschtäm & maS-tEm & CVC-CVC & 2 & 8 & 6 \\
\hline Maschtämli & maS-tEm-li: & CVC-CVC-CV & 3 & 10 & 8 \\
\hline Maschtämlirisch & maS-tEm-li:-rIS & CVC-CVC-CV-CVC & 4 & 15 & 11 \\
\hline Muschtäm & mUS-tEm & CVC-CVC & 2 & 8 & 6 \\
\hline Muschtämli & mUS-tEm-li: & CVC-CVC-CV & 3 & 10 & 8 \\
\hline Muschtämlirisch & mUS-tEm-li:-rIS & CVC-CVC-CV-CVC & 4 & 15 & 11 \\
\hline Mutläng & mUt-IEN & CVC-CVC & 2 & 7 & 6 \\
\hline Mutlängsü & mUt-IEN-sy: & CVC-CVC-CV & 3 & 9 & 8 \\
\hline Mutlängsütar & mUt-IEN-sy:-tar & CVC-CVC-CV-CVC & 4 & 12 & 11 \\
\hline Mätläng & mEt-IEN & CVC-CVC & 2 & 7 & 6 \\
\hline Mätlängsü & mEt-IEN-sy: & CVC-CVC-CV & 3 & 9 & 8 \\
\hline Mätlängsütar & mEt-IEN-sy:-tar & CVC-CVC-CV-CVC & 4 & 12 & 11 \\
\hline Wätnel & vEt-nEl & CVC-CVC & 2 & 6 & 6 \\
\hline Wätnelfo & vEt-nEl-fo: & CVC-CVC-CV & 3 & 8 & 8 \\
\hline Wätnelfosüf & vEt-nEl-fo:-zYf & CVC-CVC-CV-CVC & 4 & 11 & 11 \\
\hline Wietnel & vi:t-nEl & CVC-CVC & 2 & 7 & 6 \\
\hline Wietnelfo & vi:t-nEl-fo: & CVC-CVC-CV & 3 & 9 & 8 \\
\hline Wietnelfosüf & vi:t-nEl-fo:-zYf & CVC-CVC-CV-CVC & 4 & 12 & 11 \\
\hline Wässkahf & vEs-ka:f & CVC-CVC & 2 & 8 & 6 \\
\hline Wässkahfnai & vEs-ka:f-nai & CVC-CVC-CV & 3 & 11 & 8 \\
\hline
\end{tabular}


7. Appendices

Appendix B: Effects of word length and syllable frequency in adults with developmental stuttering

\begin{tabular}{|c|c|c|c|c|c|}
\hline Wässkahfnaixe & vEs-ka:f-nai-ks@ & CVC-CVC-CV-CCV & 4 & 13 & 11 \\
\hline Wähskahf & vE:s-ka:f & CVC-CVC & 2 & 8 & 6 \\
\hline Wähskahfnai & vE:s-ka:f-nai & CVC-CVC-CV & 3 & 11 & 8 \\
\hline Wähskahfnaixe & vE:s-ka:f-nai-ks@ & CVC-CVC-CV-CCV & 4 & 13 & 11 \\
\hline Bomkaul & bOm-kaul & CVC-CVC & 2 & 7 & 6 \\
\hline Bomkaulgu & bOm-kaul-gU & CVC-CVC-CV & 3 & 9 & 8 \\
\hline Bomkaulguzäm & bOm-kaul-gU-tsEm & CVC-CVC-CV-CCVC & 4 & 12 & 12 \\
\hline Bamkaul & bam-kaul & CVC-CVC & 2 & 7 & 6 \\
\hline Bamkaulgu & bam-kaul-gU & CVC-CVC-CV & 3 & 9 & 8 \\
\hline Bamkaulguzäm & bam-kaul-gU-tsEm & CVC-CVC-CV-CCVC & 4 & 12 & 12 \\
\hline Pantuck & pan-tUk & CVC-CVC & 2 & 7 & 6 \\
\hline Pantucklun & pan-tUk-IUn & CVC-CVC-CVC & 3 & 10 & 9 \\
\hline Pantucklunde & pan-tUk-IUn-d@ & CVC-CVC-CVC-CV & 4 & 12 & 11 \\
\hline Pehntuck & pe:n-tUk & CVC-CVC & 2 & 8 & 6 \\
\hline Pehntucklun & pe:n-tUk-IUn & CVC-CVC-CVC & 3 & 11 & 9 \\
\hline Pehntucklunde & pe:n-tUk-IUn-d@ & CVC-CVC-CVC-CV & 4 & 13 & 11 \\
\hline Patlech & pat-IEx & CVC-CVC & 2 & 7 & 6 \\
\hline Patlechtüs & pat-IEx-tYs & CVC-CVC-CVC & 3 & 10 & 9 \\
\hline Patlechtüsle & pat-IEx-tYs-I@ & CVC-CVC-CVC-CV & 4 & 12 & 11 \\
\hline Pohtlech & po:t-IEx & CVC-CVC & 2 & 8 & 6 \\
\hline Pohtlechtüs & po:t-IEx-tYs & CVC-CVC-CVC & 3 & 11 & 9 \\
\hline Pohtlechtüsle & po:t-IEx-tYs-I@ & CVC-CVC-CVC-CV & 4 & 13 & 11 \\
\hline Mähsgäl & $m E: s-g E l$ & CVC-CVC & 2 & 7 & 6 \\
\hline Mähsgälnich & mE:s-gEl-nlx & CVC-CVC-CVC & 3 & 11 & 9 \\
\hline Mähsgälnichtei & mE:s-gEl-nlx-tai & CVC-CVC-CVC-CV & 4 & 14 & 11 \\
\hline Möhsgäl & $m \&: s-g E l$ & CVC-CVC & 2 & 7 & 6 \\
\hline Möhsgälnich & m\&:s-gEl-nlx & CVC-CVC-CVC & 3 & 11 & 9 \\
\hline Möhsgälnichtei & m\&:s-gEl-nlx-tai & CVC-CVC-CVC-CV & 4 & 14 & 11 \\
\hline Bilkat & bll-kat & CVC-CVC & 2 & 6 & 6 \\
\hline Bilkatjüng & bll-kat-jYN & CVC-CVC-CVC & 3 & 10 & 9 \\
\hline Bilkatjüngheit & bll-kat-jYN-hait & CVC-CVC-CVC-CVC & 4 & 14 & 12 \\
\hline Buhlkat & bu:l-kat & CVC-CVC & 2 & 7 & 6 \\
\hline Buhlkatjüng & bu:I-kat-jYN & CVC-CVC-CVC & 3 & 11 & 9 \\
\hline Buhlkatjüngheit & bu:I-kat-jYN-hait & CVC-CVC-CVC-CVC & 4 & 15 & 12 \\
\hline Miktahn & mlk-ta:n & CVC-CVC & 2 & 7 & 6 \\
\hline Miktahnrop & mlk-ta:n-rOp & CVC-CVC-CVC & 3 & 10 & 9 \\
\hline
\end{tabular}


7. Appendices

Appendix B: Effects of word length and syllable frequency in adults with developmental stuttering

\begin{tabular}{|c|c|c|c|c|c|}
\hline Miktahnropgil & mlk-ta:n-rOp-gll & CVC-CVC-CVC-CVC & 4 & 13 & 12 \\
\hline Muktahn & mUk-ta:n & CVC-CVC & 2 & 7 & 6 \\
\hline Muktahnrop & mUk-ta:n-rOp & CVC-CVC-CVC & 3 & 10 & 9 \\
\hline Muktahnropgil & mUk-ta:n-rOp-gll & CVC-CVC-CVC-CVC & 4 & 13 & 12 \\
\hline Bitkol & blt-kOI & CVC-CVC & 2 & 6 & 6 \\
\hline Bitkolske & blt-kOl-ske: & CVC-CVC-CCV & 3 & 9 & 9 \\
\hline Bitkolskeaf & blt-kOl-ske:-af & CVC-CVC-CCV-VC & 4 & 11 & 11 \\
\hline Bähtkol & $b E: t-k O l$ & CVC-CVC & 2 & 7 & 6 \\
\hline Bähtkolske & bE:t-kOl-ske: & CVC-CVC-CCV & 3 & 10 & 9 \\
\hline Bähtkolskeaf & bE:t-kOl-ske:-af & CVC-CVC-CCV-VC & 4 & 12 & 11 \\
\hline Mährdeis & $m E: r$-dais & CVC-CVC & 2 & 8 & 6 \\
\hline Mährdeisgmi & mE:r-dais-gmi: & CVC-CVC-CCV & 3 & 11 & 9 \\
\hline Mährdeisgmidot & mE:r-dais-gmi:-dOt & CVC-CVC-CCV-CVC & 4 & 14 & 12 \\
\hline Mahrdeis & ma:r-dais & CVC-CVC & 2 & 8 & 6 \\
\hline Mahrdeisgmi & ma:r-dais-gmi: & CVC-CVC-CCV & 3 & 11 & 9 \\
\hline Mahrdeisgmidot & ma:r-dais-gmi:-dOt & CVC-CVC-CCV-CVC & 4 & 14 & 12 \\
\hline Moskün & mOs-kYn & CVC-CVC & 2 & 6 & 6 \\
\hline Moskündra & mOs-kYn-dra: & CVC-CVC-CCV & 3 & 9 & 9 \\
\hline Moskündradisch & mOs-kYn-dra:-dIS & CVC-CVC-CCV-CVC & 4 & 14 & 12 \\
\hline Müskün & my:s-kYn & CVC-CVC & 2 & 7 & 6 \\
\hline Müskündra & my:s-kYn-dra: & CVC-CVC-CCV & 3 & 10 & 9 \\
\hline Müskündradisch & my:s-kYn-dra:-dIS & CVC-CVC-CCV-CVC & 4 & 15 & 12 \\
\hline Manglig & maN-Ilg & CVC-CVC & 2 & 7 & 6 \\
\hline Mangligza & maN-Ilg-tsa: & CVC-CVC-CCV & 3 & 9 & 9 \\
\hline Mangligzasik & maN-Ilg-tsa:-zlk & CVC-CVC-CCV-CVC & 4 & 12 & 12 \\
\hline Monglig & mON-Ilg & CVC-CVC & 2 & 7 & 6 \\
\hline Mongligza & mON-Ilg-tsa: & CVC-CVC-CCV & 3 & 9 & 9 \\
\hline Mongligzasik & mON-Ilg-tsa:-zlk & CVC-CVC-CCV-CVC & 4 & 12 & 12 \\
\hline Mahnlien & ma:n-li:n & CVC-CVC & 2 & 8 & 6 \\
\hline Mahnlientrach & ma:n-li:n-trax & CVC-CVC-CCVC & 3 & 13 & 10 \\
\hline Mahnlientrachbe & ma:n-li:n-trax-b@ & CVC-CVC-CCVC-CV & 4 & 15 & 12 \\
\hline Mühnlien & my:n-li:n & CVC-CVC & 2 & 8 & 6 \\
\hline Mühnlientrach & my:n-li:n-trax & CVC-CVC-CCVC & 3 & 13 & 10 \\
\hline Mühnlientrachbe & my:n-li:n-trax-b@ & CVC-CVC-CCVC-CV & 4 & 15 & 12 \\
\hline Müttäg & $m Y t-t E: k$ & CVC-CVC & 2 & 6 & 6 \\
\hline Müttägtrom & mYt-tE:k-trOm & CVC-CVC-CCVC & 3 & 10 & 10 \\
\hline
\end{tabular}


7. Appendices

Appendix B: Effects of word length and syllable frequency in adults with developmental stuttering

\begin{tabular}{|c|c|c|c|c|c|}
\hline Müttägtromla & mYt-tE:k-trOm-la: & CVC-CVC-CCVC-CV & 4 & 12 & 12 \\
\hline Mottäg & mOt-tE:k & CVC-CVC & 2 & 6 & 6 \\
\hline Mottägtrom & mOt-tE:k-trOm & CVC-CVC-CCVC & 3 & 10 & 10 \\
\hline Mottägtromla & mOt-tE:k-trOm-la: & CVC-CVC-CCVC-CV & 4 & 12 & 12 \\
\hline Madgro & mad-gro: & CVC-CCV & 2 & 6 & 6 \\
\hline Madgrohaf & mad-gro:-haf & CVC-CCV-CVC- & 3 & 9 & 8 \\
\hline Madgrohaflei & mad-gro:-haf-lai & CVC-CCV-CVC-CV & 4 & 12 & 10 \\
\hline Mudgro & mUd-gro: & CVC-CCV & 2 & 6 & 6 \\
\hline Mudgrohaf & mUd-gro:-haf & CVC-CCV-CVC- & 3 & 9 & 8 \\
\hline Mudgrohaflei & mUd-gro:-haf-lai & CVC-CCV-CVC-CV & 4 & 12 & 10 \\
\hline Mählgrie & mE:I-gri: & CVC-CCV & 2 & 8 & 6 \\
\hline Mählgriezir & mE:I-gri:-tslr & CVC-CCV-CCVC & 3 & 11 & 10 \\
\hline Mählgriezirre & mE:I-gri:-tslr-r@ & CVC-CCV-CCVC-CV & 4 & 13 & 12 \\
\hline Mohlgrie & mo:I-gri: & CVC-CCV- & 2 & 8 & 6 \\
\hline Mohlgriezir & mo:I-gri:-tslr & CVC-CCV-CCVC & 3 & 11 & 10 \\
\hline Mohlgriezirre & mo:I-gri:-tslr-r@ & CVC-CCV-CCVC-CV & 4 & 13 & 12 \\
\hline Mochdrib & mOx-drlb & CVC-CCVC & 2 & 8 & 7 \\
\hline Mochdribgu & mOx-drlb-gU & CVC-CCVC-CV & 3 & 10 & 9 \\
\hline Mochdribguzeich & mOx-drlb-gU-tsaix & CVC-CCVC-CV-CCVC & 4 & 15 & 13 \\
\hline Mächdrib & $m E: x-d r l b$ & CVC-CCVC & 2 & 8 & 7 \\
\hline Mächdribgu & $m E: x-d r l b-g U$ & CVC-CCVC-CV & 3 & 10 & 9 \\
\hline Mächdribguzeich & mE:x-drlb-gU-tsaix & CVC-CCVC-CV-CCVC & 4 & 15 & 13 \\
\hline Bunzäs & bUn-tsEs & CVC-CCVC & 2 & 6 & 7 \\
\hline Bunzäsnat & bUn-tsEs-nat & CVC-CCVC-CVC & 3 & 9 & 10 \\
\hline Bunzäsnatke & bUn-tsEs-nat-k@ & CVC-CCVC-CVC-CV & 4 & 11 & 12 \\
\hline Bohnzäs & bo:n-tsEs & CVC-CCVC & 2 & 7 & 7 \\
\hline Bohnzäsnat & bo:n-tsEs-nat & CVC-CCVC-CVC & 3 & 10 & 10 \\
\hline Bohnzäsnatke & bo:n-tsEs-nat-k@ & CVC-CCVC-CVC-CV & 4 & 12 & 12 \\
\hline Munqual & mUn-kval & CVC-CCVC & 2 & 7 & 7 \\
\hline Munqualtock & mUn-kval-tOk & CVC-CCVC-CVC & 3 & 11 & 10 \\
\hline Munqualtockse & mUn-kval-tOk-s@ & CVC-CCVC-CVC-CV & 4 & 13 & 12 \\
\hline Mehnqual & me:n-kval & CVC-CCVC & 2 & 8 & 7 \\
\hline Mehnqualtock & me:n-kval-tOk & CVC-CCVC-CVC & 3 & 12 & 10 \\
\hline Mehnqualtockse & me:n-kval-tOk-s@ & CVC-CCVC-CVC-CV & 4 & 14 & 12 \\
\hline Fliegeus & fli:-gOys & CCV-CVC & 2 & 8 & 6 \\
\hline Fliegeuslü & fli:-gOys-ly: & CCV-CVC-CV & 3 & 10 & 8 \\
\hline
\end{tabular}


7. Appendices

Appendix B: Effects of word length and syllable frequency in adults with developmental stuttering

\begin{tabular}{|c|c|c|c|c|c|}
\hline Fliegeuslürei & fli:-gOys-ly:-rai & CCV-CVC-CV-CV & 4 & 13 & 10 \\
\hline Flahgeus & fla:-gOys & CCV-CVC & 2 & 8 & 6 \\
\hline Flahgeuslü & fla:-gOys-ly: & CCV-CVC-CV & 3 & 10 & 8 \\
\hline Flahgeuslürei & fla:-gOys-ly:-rai & CCV-CVC-CV-CV & 4 & 13 & 10 \\
\hline Prokung & pro:-kUN & CCV-CVC & 2 & 7 & 6 \\
\hline Prokunglä & pro:-kUN-IE & CCV-CVC-CV & 3 & 9 & 8 \\
\hline Prokungläto & pro:-kUN-IE-to: & CCV-CVC-CV-CV & 4 & 11 & 10 \\
\hline Prökung & pr\&:-kUN & CCV-CVC & 2 & 7 & 6 \\
\hline Prökunglä & pr\&:-kUN-IE & CCV-CVC-CV & 3 & 9 & 8 \\
\hline Prökungläto & pr\&:-kUN-IE-to: & CCV-CVC-CV-CV & 4 & 11 & 10 \\
\hline Frako & fra:-ko: & CCV-CV & 2 & 5 & 5 \\
\hline Frakoda & fra:-ko:-da & CCV-CV-CV & 3 & 7 & 7 \\
\hline Frakodason & fra:-ko:-da-zOn & CCV-CV-CV-CVC & 4 & 10 & 10 \\
\hline Fruko & fru:-ko: & CCV-CV & 2 & 5 & 5 \\
\hline Frukoda & fru:-ko:-da & CCV-CV-CV & 3 & 7 & 7 \\
\hline Frukodason & fru:-ko:-da-zOn & CCV-CV-CV-CVC & 4 & 10 & 10 \\
\hline Psylän & psy:-IEn & CCV-CVC & 2 & 6 & 6 \\
\hline Psyläntö & psy:-IEn-t\&: & CCV-CVC-CV & 3 & 8 & 8 \\
\hline Psyläntökock & psy:-IEn-t\&:-kOk & CCV-CVC-CV-CVC & 4 & 12 & 11 \\
\hline Psolän & pso:-IEn & CCV-CVC & 2 & 6 & 6 \\
\hline Psoläntö & pso:-IEn-t\&: & CCV-CVC-CV & 3 & 8 & 8 \\
\hline Psoläntökock & pso:-IEn-t\&:-kOk & CCV-CVC-CV-CVC & 4 & 12 & 10 \\
\hline Brehkal & bre:-kal & CCV-CVC & 2 & 7 & 6 \\
\hline Brehkalri & bre:-kal-rl & CCV-CVC-CV & 3 & 9 & 8 \\
\hline Brehkalridon & bre:-kal-rl-dOn & CCV-CVC-CV-CVC & 4 & 12 & 11 \\
\hline Brähkal & brE:-kal & CCV-CVC & 2 & 7 & 6 \\
\hline Brähkalri & brE:-kal-rl & CCV-CVC-CV & 3 & 9 & 8 \\
\hline Brähkalridon & brE:-kal-rl-dOn & CCV-CVC-CV-CVC & 4 & 12 & 11 \\
\hline Plagin & pla:-gln & CCV-CVC & 2 & 6 & 6 \\
\hline Plagintü & pla:-gln-ty: & CCV-CVC-CV & 3 & 8 & 8 \\
\hline Plagintükän & pla:-gln-ty:-kEn & CCV-CVC-CV-CVC & 4 & 11 & 11 \\
\hline Plügin & ply:-gln & CCV-CVC & 2 & 6 & 6 \\
\hline Plügintü & ply:-gln-ty: & CCV-CVC-CV & 3 & 8 & 8 \\
\hline Plügintükän & ply:-gln-ty:-kEn & CCV-CVC-CV-CVC & 4 & 11 & 11 \\
\hline Briesir & bri:-zlr & CCV-CVC & 2 & 7 & 6 \\
\hline Briesirtaum & bri:-zlr-taum & CCV-CVC-CVC & 3 & 11 & 9 \\
\hline
\end{tabular}


7. Appendices

Appendix B: Effects of word length and syllable frequency in adults with developmental stuttering

$\begin{array}{lllllll}\text { Briesirtaumluhf } & \text { bri:-zlr-taum-lu:f } & \text { CCV-CVC-CVC-CVC } & 4 & 15 & 12 & 11 \\ \text { Bröhsir } & \text { br\&:-zIr } & \text { CCV-CVC } & 2 & 7 & 6 & 6 \\ \text { Bröhsirtaum } & \text { br\&:-zlr-taum } & \text { CCV-CVC-CVC } & 3 & 11 & 9 & 8 \\ \text { Bröhsirtaumluhf } & \text { br\&:-zIr-taum-lu:f } & \text { CCV-CVC-CVC-CVC } & 4 & 15 & 12 & 11 \\ \text { Flürin } & \text { fly:-rln } & \text { CCV-CVC } & 2 & 6 & 6 & 6 \\ \text { Flürinsor } & \text { fly:-rln-sOr } & \text { CCV-CVC-CVC } & 3 & 9 & 9 & 10 \\ \text { Flürinsortäch } & \text { fly:-rln-sOr-tEx } & \text { CCV-CVC-CVC-CVC } & 4 & 13 & 12 & 13 \\ \text { Flärin } & \text { flE:-rln } & \text { CCV-CVC } & 2 & 6 & 6 & 6 \\ \text { Flärinsor } & \text { flE:-rln-sOr } & \text { CCV-CVC-CVC } & 3 & 9 & 9 & 10 \\ \text { Flärinsortäch } & \text { flE:-rln-sOr-tEx } & \text { CCV-CVC-CVC-CVC } & 4 & 13 & 12 & 13 \\ \text { Bransäp } & \text { bran-zEp } & \text { CCVC-CVC } & 2 & 7 & 7 & 6 \\ \text { Bransäpka } & \text { bran-zEp-ka } & \text { CCVC-CVC-CV } & 3 & 9 & 9 & 8 \\ \text { Bransäpkate } & \text { bran-zEp-ka-t@ } & \text { CCVC-CVC-CV-CV } & 4 & 11 & 11 & 8 \\ \text { Briensäp } & \text { bri:n-zEp } & \text { CCVC-CVC } & 2 & 8 & 7 & 6 \\ \text { Briensäpka } & \text { bri:n-zEp-ka } & \text { CCVC-CVC-CV } & 3 & 10 & 9 & 8 \\ \text { Briensäpkate } & \text { bri:n-zEp-ka-t@ } & \text { CCVC-CVC-CV-CV } & 4 & 12 & 11 & 8\end{array}$

Note. Syl $=$ syllable. Let $=$ letter. $\mathrm{Pho}=$ phoneme. $\mathrm{C}=$ consonant. $\mathrm{V}=$ vowel 
7. Appendices

Appendix B: Effects of word length and syllable frequency in adults with developmental stuttering

Table 4. List of intitial syllables.

\begin{tabular}{|c|c|c|c|c|c|c|c|c|c|c|c|c|}
\hline \multirow[b]{2}{*}{ ID } & \multirow[b]{2}{*}{ Ortho } & \multirow{2}{*}{$\begin{array}{l}\text { SAM- } \\
\text { PA }\end{array}$} & \multicolumn{3}{|c|}{ Frequency } & \multicolumn{5}{|c|}{ Bigram token frequency } & \multicolumn{2}{|c|}{ Nucleus } \\
\hline & & & cat & token & type & $\begin{array}{l}\text { phon., } \\
1^{\text {st }}\end{array}$ & $\begin{array}{l}\text { phon., } \\
2^{\text {nd }}\end{array}$ & $\begin{array}{l}\text { ortho., } \\
1^{\text {st }}\end{array}$ & $\begin{array}{l}\text { ortho., } \\
2^{\text {nd }}\end{array}$ & $\begin{array}{l}\text { ortho., } \\
3^{\text {rd }}\end{array}$ & tensity & PoA \\
\hline$\overline{1}$ & pie & pi: & $\mathrm{H}$ & 2,676 & 1,069 & 11,742 & 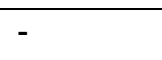 & 13,989 & 496,418 & - & $\mathrm{T}$ & $\mathrm{F}$ \\
\hline 1 & peu & pOy & $\mathrm{L}$ & 30 & 37 & 7,152 & 69,004 & 19,264 & 60,514 & - & $\mathrm{L}$ & B \\
\hline 2 & wei & vai & $\mathrm{H}$ & 16,007 & 2,551 & 83,312 & 536,665 & 143,277 & 532,784 & - & $\mathrm{L}$ & $\mathrm{F}$ \\
\hline 2 & weu & vOy & $\mathrm{L}$ & 6 & 1 & 26,740 & 69,004 & 143,277 & 60,514 & - & $\mathrm{L}$ & B \\
\hline 3 & mäh & $\mathrm{mE}:$ & $\mathrm{H}$ & 2,339 & 1,108 & 4,493 & - & 9,651 & 6,402 & - & $\mathrm{T}$ & $\mathrm{F}$ \\
\hline 3 & mä & $\mathrm{mE}$ & $\mathrm{L}$ & 0 & 13 & 26,017 & - & 9,651 & - & - & $\mathrm{L}$ & $\mathrm{F}$ \\
\hline 4 & möh & $\mathrm{m} \&:$ & $\mathrm{H}$ & 1,134 & 194 & 7,532 & - & 9,505 & 16,462 & - & $\mathrm{T}$ & $\mathrm{F}$ \\
\hline 4 & mü & $\mathrm{mY}$ & $L$ & 0 & 1 & 8,743 & - & 11,400 & - & - & $L$ & B \\
\hline 5 & müh & my: & $\mathrm{H}$ & 2,457 & 741 & 2,947 & - & 11,400 & 18,342 & - & $\mathrm{T}$ & $\mathrm{F}$ \\
\hline 5 & meu & mOy & $L$ & 98 & 125 & 3,905 & - & 128,955 & 60,514 & - & $\mathrm{L}$ & B \\
\hline 6 & muh & mu: & $\mathrm{H}$ & 4,961 & 988 & 5,895 & - & 23,447 & 10,744 & - & $\mathrm{T}$ & B \\
\hline 6 & $\mathrm{ma}$ & $\mathrm{ma}$ & L & 9 & 14 & 72,870 & - & 77,322 & - & - & $L$ & $\mathrm{~F}$ \\
\hline 7 & mur & $\mathrm{mUr}$ & $\mathrm{H}$ & 181 & 67 & 17,463 & 47,204 & 23,447 & 84,205 & - & $\mathrm{L}$ & B \\
\hline 7 & mür & $\mathrm{mYr}$ & $\mathrm{L}$ & 58 & 52 & 8,743 & 14,122 & 11,400 & 61,384 & - & $\mathrm{L}$ & B \\
\hline 8 & möhk & $m \&: k$ & $\mathrm{H}$ & 6,394 & 98 & 7,532 & 7,173 & 9,505 & 16,462 & 6,304 & $\mathrm{~T}$ & $\mathrm{~F}$ \\
\hline 8 & mähk & $\mathrm{mE}: \mathrm{k}$ & $\mathrm{L}$ & 8 & 2 & 4,493 & 2,783 & 9,651 & 6,402 & 6,304 & $\mathrm{~T}$ & $\mathrm{~F}$ \\
\hline 9 & fak & fak & $\mathrm{H}$ & 670 & 167 & 30,779 & 14,343 & 37,782 & 11,228 & - & $L$ & $\mathrm{~F}$ \\
\hline 9 & fahk & fa:k & $L$ & 0 & 1 & 11,893 & 30,778 & 37,782 & 66,914 & - & $\mathrm{T}$ & $\mathrm{F}$ \\
\hline 10 & wik & vlk & $\mathrm{H}$ & 2,551 & 347 & 66,559 & 12,737 & 128,329 & 24,156 & - & $\mathrm{L}$ & $\mathrm{F}$ \\
\hline 10 & wuk & vUk & $\mathrm{L}$ & 0 & 4 & 21,439 & 6,509 & 21,764 & 5,069 & - & $L$ & B \\
\hline 11 & mien & mi:n & $\mathrm{H}$ & 302 & 95 & 20,911 & 40,129 & 110,385 & 496,418 & $1,048,911$ & $\mathrm{~T}$ & $\mathrm{~F}$ \\
\hline 11 & muhn & mu:n & $\mathrm{L}$ & 6 & 10 & 5,895 & 15,294 & 23,447 & 10,744 & 45,650 & $\mathrm{~T}$ & B \\
\hline 12 & mas & mas & $\mathrm{H}$ & 737 & 276 & 72,870 & 144,072 & 77,322 & 116,495 & - & $\mathrm{L}$ & $\mathrm{F}$ \\
\hline 12 & mehs & me:s & $\mathrm{L}$ & 0 & 3 & 24,246 & 945 & 128,955 & 88,234 & 15,693 & $\mathrm{~T}$ & $\mathrm{~F}$ \\
\hline 13 & woch & $\mathrm{vOx}$ & $\mathrm{H}$ & 4,428 & 73 & 26,740 & 38,844 & 40,349 & 42,564 & 758,834 & $\mathrm{~L}$ & B \\
\hline 13 & wiech & vi:x & $\mathrm{L}$ & 0 & 2 & 67,460 & 794 & 128,329 & 496,418 & 29,070 & $\mathrm{~T}$ & $\mathrm{~F}$ \\
\hline 14 & fes & $\mathrm{fEs}$ & $\mathrm{H}$ & 2,949 & 639 & 105,792 & 128,781 & 64,431 & 298,880 & - & $\mathrm{L}$ & $\mathrm{F}$ \\
\hline 14 & fös & $\mathrm{f} \&: \mathrm{s}$ & $\mathrm{L}$ & 0 & 1 & 382 & 6,005 & 7,352 & 6,809 & - & $\mathrm{T}$ & $\mathrm{F}$ \\
\hline 15 & mäng & mEN & $\mathrm{H}$ & 771 & 147 & 26,017 & 19,032 & 9,651 & 15,909 & 200,537 & $\mathrm{~L}$ & $\mathrm{~F}$ \\
\hline 15 & ming & $\mathrm{mIN}$ & $\mathrm{L}$ & 13 & 7 & 89,494 & 21,124 & 110,385 & 490,581 & 200,537 & $\mathrm{~L}$ & $\mathrm{~F}$ \\
\hline 16 & mar & mar & $\mathrm{H}$ & 870 & 531 & 72,870 & 56,614 & 77,322 & 123,721 & - & $\mathrm{L}$ & $\mathrm{F}$ \\
\hline 16 & mir & $\mathrm{mlr}$ & $\mathrm{L}$ & 5 & 1 & 89,494 & 38,372 & 110,385 & 69,790 & - & $\mathrm{L}$ & $\mathrm{F}$ \\
\hline 17 & mam & mam & $\mathrm{H}$ & 494 & 19 & 72,870 & 30,908 & 77,322 & 51,491 & - & $\mathrm{L}$ & $\mathrm{F}$ \\
\hline 17 & mahm & ma:m & $\mathrm{L}$ & 0 & 1 & 26,072 & 27,442 & 77,322 & 52,594 & 25,580 & $\mathrm{~T}$ & $\mathrm{~F}$ \\
\hline
\end{tabular}


7. Appendices

Appendix B: Effects of word length and syllable frequency in adults with developmental stuttering

\begin{tabular}{|c|c|c|c|c|c|c|c|c|c|c|c|}
\hline 18 & bäs & bEs & $\mathrm{H}$ & 4,508 & 352 & 13,624 & 128,781 & 933 & 6,997 & - & L \\
\hline 18 & bähs & $b E: s$ & $\mathrm{~L}$ & 0 & 4 & 341 & 2,398 & 933 & 6,402 & 15,693 & $\mathrm{~T}$ \\
\hline 9 & fän & fEn & $\mathrm{H}$ & 1,030 & 236 & 105,792 & 121,827 & 1,372 & 15,909 & - & $\mathrm{L}$ \\
\hline 19 & fehn & fe:n & $L$ & 0 & 2 & 6,714 & 92,596 & 64,431 & 88,234 & 45,650 & $\mathrm{~T}$ \\
\hline 20 & mak & mak & $\mathrm{H}$ & 169 & 153 & 72,870 & 14,343 & 77,322 & 11,228 & - & $\mathrm{L}$ \\
\hline 20 & mäk & $\mathrm{mEk}$ & $L$ & 28 & 24 & 26,017 & 20,381 & 9,651 & 775 & - & $\mathrm{L}$ \\
\hline 21 & masch & maS & $\mathrm{H}$ & 1,295 & 201 & 72,870 & 4,330 & 77,322 & 116,495 & 230,451 & $L$ \\
\hline 21 & musch & mUS & $\mathrm{L}$ & 24 & 24 & 17,463 & 227 & 23,447 & 92,692 & 230,451 & $\mathrm{~L}$ \\
\hline 22 & mut & $\mathrm{mUt}$ & $\mathrm{H}$ & 1,511 & 111 & 17,463 & 5,296 & 23,447 & 57,591 & - & 1 \\
\hline 2 & mät & $\mathrm{mEt}$ & $L$ & 3 & 20 & 26,017 & 54,089 & 9,651 & 2,793 & - & L \\
\hline 3 & wät & vEt & $\mathrm{H}$ & 1,625 & 373 & 55,843 & 54,089 & 2,168 & 2,793 & - & L \\
\hline 3 & wiet & vi:t & $L$ & 0 & 3 & 67,460 & 44,942 & 128,329 & 496,418 & 116,167 & $\mathrm{~T}$ \\
\hline 24 & wäss & vEs & $\mathrm{H}$ & 1,592 & 187 & 55,843 & 128,781 & 2,168 & 6,997 & 67,494 & L \\
\hline 24 & wähs & $v E: S$ & $\mathrm{~L}$ & 0 & 4 & 10,480 & 2,398 & 2,168 & 6,402 & 15,693 & $\mathrm{~T}$ \\
\hline 25 & bom & bOm & $\mathrm{H}$ & 691 & 169 & 6,044 & 21,684 & 10,672 & 30,905 & - & $\mathrm{L}$ \\
\hline 25 & bam & bam & $L$ & 0 & 5 & 62,924 & 30,908 & 29,141 & 51,491 & - & $L$ \\
\hline 26 & pan & pan & $\mathrm{H}$ & 852 & 285 & 18,105 & 209,134 & 23,061 & 252,221 & - & $\mathrm{L}$ \\
\hline 6 & pehn & pe:n & $L$ & 0 & 3 & 3,924 & 92,596 & 19,264 & 88,234 & 45,650 & $\mathrm{~T}$ \\
\hline 27 & pat & pat & $\mathrm{H}$ & 579 & 375 & 18,105 & 79,519 & 23,061 & 105,550 & - & L \\
\hline 27 & poht & po:t & $L$ & 0 & 1 & 13,935 & 28,661 & 20,999 & 20,744 & 156,005 & $\mathrm{~T}$ \\
\hline 28 & mähs & $\mathrm{mE}: \mathrm{s}$ & $\mathrm{H}$ & 399 & 39 & 4,493 & 2,398 & 9,651 & 6,402 & 15,693 & $\mathrm{~T}$ \\
\hline 28 & möhs & $m \&: s$ & $L$ & 0 & 2 & 7,532 & 6,005 & 9,505 & 16,462 & 15,693 & $\mathrm{~T}$ \\
\hline 29 & bil & bll & $\mathrm{H}$ & 4,539 & 864 & 30,530 & 26,399 & 38,142 & 57,282 & - & L \\
\hline 29 & buhl & bu:l & $L$ & 0 & 5 & 2,545 & 8,553 & 14,369 & 10,744 & 52,981 & . \\
\hline 30 & mik & mlk & $\mathrm{H}$ & 158 & 57 & 89,494 & 12,737 & 110,385 & 24,156 & - & L \\
\hline 30 & muk & mUk & $L$ & 0 & 52 & 17,463 & 6,509 & 23,447 & 5,069 & - & L \\
\hline 31 & bit & blt & $\mathrm{H}$ & 2,360 & 196 & 30,530 & 90,866 & 38,142 & 212,009 & - & L \\
\hline 31 & bäht & $b E: t$ & $L$ & 0 & 2 & 341 & 14,046 & 933 & 6,402 & 156,005 & $\mathrm{~T}$ \\
\hline 32 & mähr & $\mathrm{mE}: \mathrm{r}$ & $\mathrm{H}$ & 126 & 47 & 4,493 & 22,105 & 9,651 & 6,402 & 121,476 & $\mathrm{~T}$ \\
\hline 32 & mahr & ma:r & $L$ & 4 & 2 & 26,072 & 95,897 & 77,322 & 52,594 & - & $\mathrm{T}$ \\
\hline 33 & mos & $\mathrm{mOs}$ & $\mathrm{H}$ & 127 & 44 & 3,905 & 12,471 & 17,574 & 23,236 & - & L \\
\hline 3 & müs & my:s & $\mathrm{L}$ & 0 & 2 & 2,947 & 1,977 & 11,400 & 9,053 & - & $r$ \\
\hline 4 & mang & maN & $\mathrm{H}$ & 359 & 87 & 72,870 & 31,837 & 77,322 & 252,221 & 200,537 & $\mathrm{~L}$ \\
\hline 4 & mong & $\mathrm{mON}$ & $L$ & 30 & 32 & 3,905 & 503 & 17,574 & 148,465 & 200,537 & $L$ \\
\hline 35 & mahn & ma:n & $\mathrm{H}$ & 360 & 110 & 26,072 & 14,077 & 77,322 & 52,594 & 45,650 & $\mathrm{~T}$ \\
\hline 35 & mühn & my:n & $L$ & 2 & 8 & 2,947 & 1,809 & 11,400 & 18,342 & 45,650 & $\mathrm{~T}$ \\
\hline 36 & müt & $\mathrm{mYt}$ & $\mathrm{H}$ & 181 & 97 & 8,743 & 3,881 & 11,400 & 5,605 & - & $\mathrm{L}$ \\
\hline 36 & mot & $\mathrm{mOt}$ & $L$ & 83 & 22 & 3,905 & 3,916 & 17,574 & 19,321 & - & L \\
\hline & mad & mad & $\mathrm{H}$ & 117 & 51 & 72,870 & 2,275 & 77,322 & 16,652 & - & L \\
\hline
\end{tabular}


7. Appendices

Appendix B: Effects of word length and syllable frequency in adults with developmental stuttering

\begin{tabular}{|c|c|c|c|c|c|c|c|c|c|c|c|}
\hline 37 & mud & $\mathrm{mUd}$ & $\mathrm{L}$ & 1 & 2 & 17,463 & 28 & 23,447 & 4,489 & & $\mathrm{~L}$ \\
\hline 38 & mähl & $\mathrm{mE}: \mathrm{I}$ & $\mathrm{H}$ & 119 & 27 & 4,493 & 3,801 & 9,651 & 6,402 & 52,981 & $\mathrm{~T}$ \\
\hline 38 & mohl & mo:l & $\mathrm{L}$ & 1 & 1 & 13,748 & 25,884 & 17,574 & 20,744 & 52,981 & $\mathrm{~T}$ \\
\hline 39 & moch & $\mathrm{mOx}$ & $\mathrm{H}$ & 371 & 8 & 3,905 & 38,844 & 17,574 & 42,564 & 758,834 & $\mathrm{~L}$ \\
\hline 39 & mäch & $\mathrm{mE}: \mathrm{x}$ & $\mathrm{L}$ & 0 & 17 & 4,493 & 5,768 & 9,651 & 3,407 & 758,834 & $\mathrm{~T}$ \\
\hline 40 & bun & bUn & $\mathrm{H}$ & 4,124 & 276 & 11,851 & 241,149 & 14,369 & 392,058 & - & $\mathrm{L}$ \\
\hline 40 & bohn & bo:n & $\mathrm{L}$ & 0 & 7 & 6,014 & 63,118 & 10,672 & 20,744 & 45,650 & $\mathrm{~T}$ \\
\hline 41 & mun & mUn & $\mathrm{H}$ & 205 & 101 & 17,463 & 241,149 & 23,447 & 392,058 & - & $\mathrm{L}$ \\
\hline 1 & mehn & me:n & $\mathrm{L}$ & 92 & 1 & 24,246 & 92,596 & 128,955 & 88,234 & 45,650 & $\mathrm{~T}$ \\
\hline 12 & flie & fli: & $\mathrm{H}$ & 732 & 421 & 13,361 & 62,046 & 13,351 & 169,490 & 496,418 & $\mathrm{~T}$ \\
\hline 42 & flah & fla: & $\mathrm{L}$ & 6 & 8 & 13,361 & 20,462 & 13,351 & 74,834 & 52,594 & $\mathrm{~T}$ \\
\hline 43 & pro & pro: & $\mathrm{H}$ & 16,467 & 1,963 & 51,241 & 39,364 & 50,691 & 50,416 & - & $\mathrm{T}$ \\
\hline 43 & prö & pr\&: & $\mathrm{L}$ & 0 & 5 & 51,241 & 5,113 & 50,691 & 21,016 & - & $\mathrm{T}$ \\
\hline 44 & fra & fra: & $\mathrm{H}$ & 7,254 & 323 & 43,130 & 46,447 & 43,092 & 110,357 & - & $\mathrm{T}$ \\
\hline 44 & fru & fru: & $\mathrm{L}$ & 3 & 18 & 43,130 & 11,803 & 43,092 & 59,471 & - & $T$ \\
\hline 45 & psy & psy: & $\mathrm{H}$ & 487 & 137 & 8,542 & 2,433 & 1,516 & 2,621 & - & 1 \\
\hline 45 & pso & pso: & $\mathrm{L}$ & 6 & 9 & 8,542 & 4,193 & 1,516 & 88,483 & - & $\mathrm{T}$ \\
\hline 46 & breh & bre: & $\mathrm{H}$ & 592 & 27 & 25,803 & 35,255 & 26,353 & 254,867 & 88,234 & $\mathrm{~T}$ \\
\hline 46 & bräh & brE: & $\mathrm{L}$ & 12 & 37 & 25,803 & 8,254 & 26,353 & 7,389 & 6,402 & $\mathrm{~T}$ \\
\hline 47 & pla & pla: & $\mathrm{H}$ & 790 & 162 & 13,664 & 20,462 & 9,625 & 74,834 & - & $\mathrm{T}$ \\
\hline 47 & plü & ply: & $\mathrm{L}$ & 0 & 3 & 13,664 & 2,019 & 9,625 & 5,569 & - & $\mathrm{T}$ \\
\hline 48 & brie & bri: & $\mathrm{H}$ & 1,494 & 267 & 25,803 & 40,866 & 26,353 & 97,513 & 496,418 & $\mathrm{~T}$ \\
\hline 48 & bröh & br\&: & $\mathrm{L}$ & 6 & 44 & 25,803 & 5,113 & 26,353 & 21,016 & 16,462 & $\mathrm{~T}$ \\
\hline 49 & flü & fly: & $\mathrm{H}$ & 365 & 104 & 13,361 & 2,019 & 13,351 & 5,569 & - & $\mathrm{T}$ \\
\hline 49 & flä & $\mathrm{flE}:$ & $\mathrm{L}$ & 14 & 30 & 13,361 & 7,792 & 13,351 & 5,079 & - & $\mathrm{T}$ \\
\hline 50 & bran & bran & $\mathrm{H}$ & 210 & 170 & 25,803 & 118,040 & 26,353 & 110,357 & 252,221 & $\mathrm{~L}$ \\
\hline 50 & brien & bri:n & $\mathrm{L}$ & 0 & 1 & 25,803 & 40,866 & 26,353 & 97,513 & 496,418 & $\mathrm{~T}$ \\
\hline
\end{tabular}

Note. ID represents the ID of high- and low-frequency pseudoword pairs. cat = category. phon. $=$ phonological. ortho $=$ orthographic. $\mathrm{PoA}=$ place of articulation. $\mathrm{H}=$ high. $\mathrm{L}=$ low. $\mathrm{T}=$ tense. $\mathrm{L}=$ lax. $\mathrm{F}=$ front. $\mathrm{B}=$ back. 


\section{Appendices}

Appendix B: Effects of word length and syllable frequency in adults with developmental stuttering

Table 5. Likelihood ratio tests of additional factors.

\begin{tabular}{|c|c|c|c|}
\hline Model & Additional factor & Chi square (DF) & p-value \\
\hline \multirow{8}{*}{$\begin{array}{l}\text { Stuttering events overall } \\
\text { syllable positions }\end{array}$} & stuttering severity & $11.2(1)$ & $<.001$ \\
\hline & reading performance (SLRT) & $0.051(1)$ & .821 \\
\hline & vowel length & $1.75(1)$ & .186 \\
\hline & $1^{\text {st }}$ phonetic bigram frequency & 1.61 & .205 \\
\hline & $2^{\text {nd }}$ phonetic bigram frequency & 3.98 & .046 \\
\hline & random effect of $2^{\text {nd }}$ phoneme & $7.23(2)$ & .027 \\
\hline & bigram frequency and stuttering & & \\
\hline & severity & & \\
\hline \multirow{6}{*}{$\begin{array}{l}\text { Stuttering events in first } \\
\text { syllable position }\end{array}$} & stuttering severity & $12.63(1)$ & $<.001$ \\
\hline & reading performance (SLRT) & $0.11(1)$ & .739 \\
\hline & vowel length & $12.65(1)$ & $<.001$ \\
\hline & $1^{\text {st }}$ phonetic bigram frequency & $0.006 \quad(1)$ & .939 \\
\hline & $2^{\text {nd }}$ phonetic bigram frequency & $0.726 \quad(1)$ & .394 \\
\hline & $\begin{array}{l}\text { random effect of stuttering } \\
\text { severity and vowel length }\end{array}$ & $7.937(2)$ & .019 \\
\hline \multirow{6}{*}{$\begin{array}{l}\text { response errors over all } \\
\text { syllable positions }\end{array}$} & stuttering severity & $0.614(1)$ & .433 \\
\hline & reading performance (SLRT) & $8.31(1)$ & .004 \\
\hline & vowel length & $0.023(1)$ & .879 \\
\hline & $1^{\text {st }}$ phonetic bigram frequency & $0.802(1)$ & .371 \\
\hline & $2^{\text {nd }}$ phonetic bigram frequency & $0.281(1)$ & .596 \\
\hline & $\begin{array}{l}\text { random effect of reading } \\
\text { performance }\end{array}$ & $0.000(1)$ & .999 \\
\hline \multirow{6}{*}{$\begin{array}{l}\text { response errors in first } \\
\text { syllable positions }\end{array}$} & stuttering severity & $0.0008(1)$ & .977 \\
\hline & reading performance (SLRT) & $7.96(1)$ & .005 \\
\hline & vowel length & $0.015(1)$ & .904 \\
\hline & $1^{\text {st }}$ phonetic bigram frequency & $0.21(1)$ & .647 \\
\hline & $2^{\text {nd }}$ phonetic bigram frequency & $0.069(1)$ & .793 \\
\hline & $\begin{array}{l}\text { random effect of reading } \\
\text { performance }\end{array}$ & $1.549(1)$ & .213 \\
\hline
\end{tabular}




\section{Appendices}

Appendix B: Effects of word length and syllable frequency in adults with developmental stuttering

Table 6. Minimum and maximum estimates derived from model stability analysis of stuttering events

\begin{tabular}{|c|c|c|c|c|}
\hline $\begin{array}{l}\text { stuttering } \\
\text { probability }\end{array}$ & Model terms & orig & $\min$ & $\max$ \\
\hline \multirow{12}{*}{$\begin{array}{l}\text { Stuttering } \\
\text { overall } \\
\text { positions }\end{array}$} & FE: (Intercept) & -2.21 & -2.34 & -2.03 \\
\hline & FE: pseudoword length & 0.53 & 0.50 & 0.58 \\
\hline & FE: syllable frequency & -0.15 & -0.19 & -0.11 \\
\hline & FE: stuttering severity & 1.32 & 1.11 & 1.49 \\
\hline & FE: second phonetic bigram frequency & 0.03 & -0.04 & 0.08 \\
\hline & FE: pseudoword length : syllable frequency & 0.06 & 0.02 & 0.09 \\
\hline & RE: subject @ intercept & 1.38 & 1.19 & 1.54 \\
\hline & $\begin{array}{l}\text { RE: subject @ second phonetic bigram } \\
\text { frequency }{ }^{a}\end{array}$ & 0.26 & 0.22 & 0.35 \\
\hline & $\begin{array}{l}\text { RE: subject @ second phonetic bigram } \\
\text { frequency }{ }^{b}\end{array}$ & 0.79 & 0.66 & 0.99 \\
\hline & RE: pseudoword pair @ (Intercept) & 0.65 & 0.58 & 0.73 \\
\hline & RE: pseudoword pair @ stuttering severity a & 0.34 & 0.19 & 0.54 \\
\hline & RE: pseudoword pair @ stuttering severity b & -0.71 & -1 & -0.61 \\
\hline \multirow{12}{*}{$\begin{array}{l}\text { Stuttering events at } \\
\text { first syllable }\end{array}$} & FE: (Intercept) & -3.66 & -4.02 & -3.45 \\
\hline & FE: pseudoword length & 0.29 & 0.24 & 0.35 \\
\hline & FE: syllable frequency & -0.21 & -0.30 & -0.15 \\
\hline & FE: vowel length & -0.19 & -0.42 & 0.01 \\
\hline & FE: stuttering severity & 1.87 & 1.53 & 2.14 \\
\hline & FE: pseudoword length : syllable frequency & 0.13 & 0.08 & 0.19 \\
\hline & RE: subject @ (Intercept) & 1.67 & 1.49 & 1.86 \\
\hline & RE: subject @ vowel length a & 0.84 & 0.61 & 0.97 \\
\hline & RE: subject @ vowel length b & -0.52 & -0.83 & -0.39 \\
\hline & RE: pseudoword pairs @ (Intercept) & 0.78 & 0.68 & 1.01 \\
\hline & RE: pseudoword pairs @ stuttering severity a & 0.66 & 0.36 & 0.92 \\
\hline & RE: pseudoword pairs @ stuttering severity b & -0.87 & -0.99 & -0.79 \\
\hline
\end{tabular}

Note. Model stability was investigated by excluding subjects one at a time from the data and comparing the model estimates derived for these subsets of the data with those derived for the full data set. This indicated no influential subjects to exist. arandom slope. brandom slope and correlations. $\mathrm{FE}=$ fixed effects. $\mathrm{RE}=$ random effects. 


\section{Appendices}

Appendix B: Effects of word length and syllable frequency in adults with developmental stuttering

Table 7. Minimum and maximum estimates of model stability analysis of response accuracy

\begin{tabular}{|c|c|c|c|c|}
\hline $\begin{array}{l}\text { error } \\
\text { probability }\end{array}$ & Model terms & orig & $\min$ & $\max$ \\
\hline \multirow{14}{*}{$\begin{array}{l}\text { errors overall } \\
\text { syllable } \\
\text { positions }\end{array}$} & FE: (Intercept) & -2.47 & -2.52 & -2.44 \\
\hline & FE: pseudoword length & 0.55 & 0.53 & 0.58 \\
\hline & FE: syllable frequency & 0.06 & 0.02 & 0.10 \\
\hline & FE: group & -0.12 & -0.22 & -0.06 \\
\hline & FE: reading performance & -0.31 & -0.35 & -0.26 \\
\hline & FE: syllable frequency : pseudoword length & 0.12 & 0.08 & 0.18 \\
\hline & FE: group : pseudoword length & -0.03 & -0.06 & 0.02 \\
\hline & FE: syllable frequency : group & 0.03 & -0.03 & 0.12 \\
\hline & FE: group: syllable frequency : pseudoword length & 0.08 & 0.01 & 0.14 \\
\hline & RE: subject @ (Intercept) & 0.49 & 0.45 & 0.51 \\
\hline & RE: pseudoword pairs @ (Intercept) & 0.61 & 0.55 & 0.64 \\
\hline & RE: pseudoword pairs @ group a & 0.49 & 0.37 & 0.59 \\
\hline & RE: pseudoword pairs @ pseudoword length a & 0.39 & 0.36 & 0.43 \\
\hline & RE: pseudoword pairs @ syllable frequency a & 0.44 & 0.37 & 0.49 \\
\hline \multirow{16}{*}{$\begin{array}{l}\text { errors at first } \\
\text { syllable }\end{array}$} & FE: (Intercept) & -4.17 & -4.26 & -4.11 \\
\hline & FE: pseudoword length & 0.21 & 0.17 & 0.27 \\
\hline & FE: syllable frequency & 0.13 & 0.04 & 0.24 \\
\hline & FE: group & -0.15 & -0.29 & -0.04 \\
\hline & FE: reading performance & -0.46 & -0.52 & -0.38 \\
\hline & FE: syllable frequency : pseudoword length & 0.34 & 0.27 & 0.43 \\
\hline & FE: syllable frequency : group & -1.38 & -1.65 & -1.17 \\
\hline & FE: group : pseudoword length & 0.18 & 0.08 & 0.24 \\
\hline & FE: group : syllable frequency : pseudoword length & 0.24 & 0.09 & 0.42 \\
\hline & RE: subject @ (Intercept) & 0.64 & 0.57 & 0.68 \\
\hline & RE: pseudoword pairs @ (Intercept) & 0.88 & 0.76 & 0.92 \\
\hline & RE: pseudoword pairs @ syllable frequency a & 1.38 & 1.28 & 1.52 \\
\hline & RE: pseudoword pairs @ group a & 1.04 & 0.70 & 1.26 \\
\hline & $\begin{array}{l}\text { RE: pseudoword pairs @ syllable frequency @ } \\
\text { group }^{a}\end{array}$ & 0.84 & 0.69 & 0.94 \\
\hline & RE: pseudoword pairs @ syllable frequency b & -0.12 & -0.27 & 0.15 \\
\hline & RE: pseudoword pairs @ group b & -0.10 & -0.26 & -0.001 \\
\hline
\end{tabular}

Note. Model stability was investigated by excluding subjects one at a time from the data and comparing the model estimates derived for these subsets of the data with those derived for the full

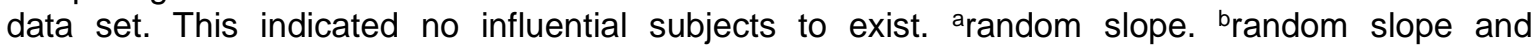
correlations. $\mathrm{FE}=$ fixed effects. $\mathrm{RE}=$ random effects. 
7. Appendices

Appendix B: Effects of word length and syllable frequency in adults with developmental stuttering

Table 8. Mean of stuttered events and response errors in initial syllable position per participant.

\begin{tabular}{|c|c|c|c|c|c|c|c|c|c|c|c|c|c|}
\hline ID & $\mathbf{G}$ & $\begin{array}{l}\text { SSI } \\
\text { R+S }\end{array}$ & $\begin{array}{c}\text { SLRT } \\
\text { PW }\end{array}$ & $\begin{array}{l}\text { SE } \\
\text { HF }\end{array}$ & $\begin{array}{l}\text { SE } \\
\text { LF }\end{array}$ & $\begin{array}{c}\text { SE } \\
\text { 2syl }\end{array}$ & $\begin{array}{c}\text { SE } \\
\text { 3syl }\end{array}$ & $\begin{array}{l}\text { SE } \\
4 s y l\end{array}$ & $\begin{array}{l}\text { RE } \\
\text { HF }\end{array}$ & $\begin{array}{l}\text { RE } \\
\text { LF }\end{array}$ & $\begin{array}{c}\text { RE } \\
\text { 2syl }\end{array}$ & $\begin{array}{c}\text { RE } \\
\text { 3syl }\end{array}$ & $\begin{array}{c}\text { RE } \\
4 \text { syl }\end{array}$ \\
\hline S01 & $S$ & 3 & 73 & 0 & 1.09 & 0 & 1.43 & 0 & 2.08 & 3.12 & 0 & 1.43 & 6.25 \\
\hline SO2 & $S$ & 4 & 59 & 2.38 & 1.19 & 0 & 3.45 & 2.78 & 2.35 & 2.35 & 1.32 & 0 & 7.50 \\
\hline SO3 & $S$ & 6 & 47 & 2.38 & 0 & 0 & 0 & 5.56 & 14.81 & 9.26 & 14.58 & 10 & 10 \\
\hline$S 04$ & $S$ & 8 & 68 & 2.22 & 0 & 0 & 0 & 5.00 & 9.62 & 5.77 & 8.00 & 11.11 & 0 \\
\hline S05 & $S$ & 16 & 48 & 25.81 & 32.26 & 17.65 & 43.75 & 41.67 & 12.90 & 22.58 & 13.89 & 25.00 & 20 \\
\hline S06 & $S$ & 14 & 45 & 10.64 & 8.51 & 10.71 & 8.33 & 7.14 & 0 & 2.56 & 0 & 0 & 7.14 \\
\hline S07 & $S$ & 5 & 71 & 0 & 1.37 & 1.72 & 0 & 0 & 6.10 & 6.10 & 6.25 & 5.17 & 7.14 \\
\hline 508 & $S$ & 6 & 75 & 1.12 & 0 & 0 & 1.56 & 0 & 2.22 & 0 & 1.52 & 1.56 & 0 \\
\hline 509 & $S$ & 4 & 76 & 0 & 0 & 0 & 0 & 0 & 7.35 & 2.94 & 1.67 & 7.14 & 8.82 \\
\hline$S 10$ & $S$ & 15 & 74 & 88.00 & 82.67 & 76.56 & 86.36 & 97.62 & 0 & 25.00 & 16.67 & 0 & - \\
\hline$S 11$ & $S$ & 11 & 52 & 40.48 & 27.38 & 26.25 & 40.38 & 41.67 & 0 & 2.44 & 2.08 & 0 & 0 \\
\hline$S 12$ & $S$ & 11 & 55 & 0 & 0 & 0 & 0 & 0 & 0 & 0 & 0 & 0 & 0 \\
\hline$S 13$ & $S$ & 9 & 72 & 20.59 & 23.53 & 14.29 & 18.52 & 35.00 & 4.08 & 0 & 0 & 2.38 & 4.55 \\
\hline$S 14$ & $S$ & 6 & 60 & 2.17 & 0 & 0 & 3.33 & 0 & 4.26 & 0 & 2.50 & 3.33 & 0 \\
\hline$S 15$ & $S$ & 15 & 46 & 1.72 & 1.72 & 1.85 & 2.63 & 0 & 6.06 & 9.09 & 6.67 & 5.00 & 12.50 \\
\hline$S 16$ & $S$ & 12 & 64 & 38.46 & 36.54 & 47.50 & 30 & 33.33 & 0 & 0 & 0 & 0 & 0 \\
\hline$S 17$ & $S$ & 17 & 76 & 26.09 & 17.39 & 14.58 & 25.00 & 35.00 & 3.12 & 0 & 0 & 0 & 8.33 \\
\hline$S 18$ & $S$ & 9 & 92 & 25.64 & 16.67 & 17.86 & 18.97 & 28.57 & 0 & 3.70 & 0 & 2.27 & 3.85 \\
\hline$S 19$ & $S$ & 11 & 72 & 17.53 & 12.37 & 16.67 & 16.13 & 11.67 & 2.67 & 1.33 & 0 & 2.08 & 4.00 \\
\hline$S 20$ & $F$ & 0 & 64 & - & - & - & - & - & 3.80 & 6.33 & 3.23 & 1.67 & 13.89 \\
\hline$S 21$ & $F$ & 2 & 71 & - & - & - & - & - & 3.23 & 4.30 & 4.55 & 6.45 & 0 \\
\hline s22 & $F$ & 2 & 61 & - & - & - & - & - & 1.43 & 5.71 & 3.33 & 2.17 & 5.88 \\
\hline$S 23$ & $F$ & 4 & 61 & - & - & - & - & - & 4.05 & 8.11 & 5.00 & 2.00 & 13.16 \\
\hline S24 & $F$ & 2 & 77 & - & - & - & - & - & 5.75 & 6.90 & 7.35 & 5.17 & 6.25 \\
\hline S25 & $F$ & 2 & 79 & - & - & - & - & - & 1.00 & 0 & 0 & 0 & 1.92 \\
\hline S26 & $F$ & 2 & 57 & - & - & - & - & - & 2.94 & 4.90 & 1.43 & 5.41 & 5.00 \\
\hline
\end{tabular}




\section{Appendices}

Appendix B: Effects of word length and syllable frequency in adults with developmental stuttering

$\begin{array}{llllllllllllll}S 27 & F & 2 & 106 & - & - & - & - & - & 0 & 1.10 & 0 & 0 & 2.38 \\ S 28 & F & 4 & 86 & - & - & - & - & - & 5.00 & 10 & 8.82 & 4.35 & 8.70 \\ S 29 & F & 4 & 75 & - & - & - & - & - & 3.45 & 3.45 & 4.17 & 1.79 & 4.35 \\ S 30 & F & 2 & 85 & - & - & - & - & - & 4.30 & 2.15 & 4.29 & 3.12 & 1.92 \\ S 31 & F & 0 & 65 & - & - & - & - & - & 2.27 & 3.41 & 1.52 & 4.29 & 2.50 \\ S 32 & F & 2 & 64 & - & - & - & - & - & 5.26 & 5.26 & 5.17 & 4.76 & 5.77 \\ S 33 & F & 2 & 97 & - & - & - & - & - & 1.12 & 1.12 & 1.47 & 0 & 1.61 \\ S 34 & F & 2 & 81 & - & - & - & - & - & 1.02 & 2.04 & 0 & 0 & 5.17 \\ S 35 & F & 4 & 77 & - & - & - & - & - & 1.04 & 1.04 & 1.43 & 1.56 & 0 \\ S 36 & F & 0 & 73 & - & - & - & - & - & 5.56 & 2.78 & 3.12 & 5.26 & 4.76 \\ S 37 & F & 0 & 102 & - & - & - & - & - & 1.23 & 0 & 1.61 & 0 & 0 \\ S 38 & F & 2 & 87 & - & - & - & - & - & 3.19 & 3.19 & 4.29 & 1.67 & 3.45\end{array}$

Note. $\mathrm{G}=$ Group. $\mathrm{S}=$ stuttering. $\mathrm{F}=$ fluent. $\mathrm{SSI} \mathrm{R}+\mathrm{L}=$ Sum of $\mathrm{SSI}$ scores for reading a text and for a spontaneous speech sample. SLRT-II PW = number of pseudowords read within one minute. SE = stuttering event. $\mathrm{RE}=$ response error. $\mathrm{HF}=$ high-frequency pseudowords. LF: low-frequency pseudowords. 2 syl $=2$-syllabic pseudoword. 3 syl $=3$-syllabic pseudoword. 4 syl $=4$-syllabic pseudoword. 


\section{Appendices}

Appendix C: Fluent speech: neural basis of sensorimotor plasticity in developmental stuttering

\section{Appendix C: Fluent speech: neural basis of sensorimotor plasticity in developmental stuttering}

\section{Supplementary materials and methods}

Fiber tract segmentation with tract-specific probability maps, waypoint and exclusion masks and anatomical endpoint filtering

A three-step procedure implemented in pyAFQ (https://github.com/yeatmanlab/pyAFQ) was used to segment the superior longitudinal fasciculus (SLF), the frontal aslant tract (FAT), and the tract connection LMC and IFG (LMC-to-IFG).

(1) The whole brain tractogram was filtered with tract-specific probability maps with a probability of 0.25 . The probability maps for the left and right SLF were provided with pyAFQ software. The maps for the FAT were taken from FMRIB Software Library, (FSL 6.0) providing the population percentage tract atlases of the left and right FAT, respectively (Warrington et al., 2020). The maps for LMC-to-IFG were generated from the current data set. To this end, diffusion-weighted (d)MRI images were processed with tools from the FMRIB Software Library, FSL, http://www.fmrbi.ox.ac.uk/fsl// (Jenkinson et al., 2012). Images were corrected for eddy currents and head motion by using affine registration to the non-diffusion volumes. Probabilistic tractography was performed in the individual dMRI data space. We computed voxel-wise estimates of the fiber orientation distribution of up to two fiber orientations with the FSL function bedpost (Behrens et al., 2007; Jbabdi et al., 2012). Seed mask and target masks were $3 \mathrm{~mm}$ spheres derived from the MNI coordinates that were shifted to the nearest white matter voxel in the FMRIB58_FA standard space image (left IFG: -55, 6, 10 and left LMC: -47, -4, 34; right IFG: 54, 10, 7 and right LMC: 45, -3, 35) and warped to the native dMRI (Andersson Jesper LR et al., 2007). We used modified Euler streamlining, distance correction, and 100,000 samples per voxel within the FSL function probtrackx 2 with three pairs of seed and target mask. Target mask determined both waypoint and termination mask to compute the structural connectivity between left hemispheric brain regions. The connectivity index was determined from the number of 


\section{Appendices}

Appendix C: Fluent speech: neural basis of sensorimotor plasticity in developmental stuttering

sample streamlines from each seed that reached the target. We normalized the connectivity index by dividing the logarithm of the number of streamlines from a given seed that reached the target (i.e., numeric output of the tractography algorithm given as waytotal) by the logarithm of the product of the number of generated sample streamlines in each seed voxel $(100,000)$ and the number of voxels in the seed mask, $n=19$. The logarithmic scaling transformed the connectivity index into a normally distributed variable with a range between 0 and 1. Finally, individual tractograms were warped to the 1mm FMRIB58_FA standard template, binarized, accumulated within one volume and normalized to represent a population percentage tract atlases scaling from 0 to 1.

(2) In a next step filtered tractograms were additionally filtered with waypoint and exclusion masks. A sagittal mask through the corpus callosum was used for every tract segmentation to exclude streamlines crossing hemispheres. In addition, the waypoint and exclusion masks for the left and right SLF were provided with pyAFQ software. The waypoint masks for the left and right FAT were taken from https://github.com/yeatmanlab/AFQ/tree/master/ aslant/ROIs (6). The waypoint masks for the left and right LMC-to-IFG were manually drawn on the FSL_HCP1065_FA_1mm template overlaid with the custom tract-specific probability maps as shown here in appendix 3, Figure 1. Waypoint masks covered an area of $15 \times 15 \mathrm{~mm}^{2}$ and exclusion masks covered an area of $60 \times 60 \mathrm{~mm}^{2}$ with coordinates centered at $x=-50, y=10, z=12$ (left waypoint mask 1 ), $x=-45, y=-3, z=33$ (left waypoint mask 2), $x=-23, y=-2, z=34$ (left exclusion mask), $x=50, y=9, z=12$ (right waypoint mask 1), $x=43, y=-3, z=33$ (right waypoint mask 2), and $x=26, y=-2, z=34$ (right exclusion mask).

(3) Native anatomy was skullstripped, tissue segmented and anatomically labeled with FreeSurfer 6.0 (https://surfer.nmr.mgh.harvard.edu/). This enabled anatomical endpoint filtering, making sure that segmented streamlines terminate in defined cortical regions. 


\section{Appendices}

Appendix C: Fluent speech: neural basis of sensorimotor plasticity in developmental stuttering

\section{Supplementary discussion}

Changed rs-fMRI connectivity in control groups

While participants of the intervention group intensively practiced a new speech technique for 11 months, stuttering controls and fluent controls lived their routine lives. However, post hoc results of the global ANCOVA revealed a significant group difference in left IFG to left SMG connectivity change of stuttering controls compared with fluent controls (Table 12). The groups of this study differed significantly in age, with older individuals in the stuttering control group compared to fluent controls and participants of the intervention group. For this reason, we included age as a variable of no interest in our statistical models. When the global ANOVA was recalculated without age as nuisance variable, only the left IFG to left LMC rs-fMRI connectivity remained significant with conservative as well as liberal correction levels. In addition, age only showed an impact on left IFG to SMG rs-fMRI-connectivity change in PDS- compared with PDS+ and HC (appendix 3, Table 7). However, this influence had opposite directions. In stuttering controls, the connectivity values of left IFG to SMG increased with increasing age while participants of the intervention group and fluent controls showed the opposite association, i.e. connectivity decreases with increasing age (appendix 3, Figure 3). Thus, age seems to influence rs-fMRI connectivity changes between left IFG and left SMG in a complex manner. Literature on resting-state networks supports the idea that age contributes to the decline of high-order cognitive networks such as the dorsal attention network and the frontoparietal control network (Andrews-Hanna et al., 2007; Tomasi \& Volkow, 2012). Already at middle age a decline in network connectivity becomes evident (Evers et al., 2012; Siman-Tov et al., 2016). Such findings imply that the decline in rs-fMRI connectivity in healthy controls observed here does indeed relate to aging. 
7. Appendices

Appendix C: Fluent speech: neural basis of sensorimotor plasticity in developmental stuttering

Table 1: Demographic information per participant.

\begin{tabular}{|c|c|c|c|c|c|c|c|}
\hline ID & Group & Sex & $\begin{array}{l}\text { Age, } \\
\text { years }\end{array}$ & Education & Handedness (LQ) & $\begin{array}{c}\text { MRI } \\
\text { Interval, } \\
\text { months }\end{array}$ & $\begin{array}{c}\text { Stuttering onset, } \\
\text { years }\end{array}$ \\
\hline P01 & PDS+ & male & 39.58 & 2 & 81.81 & 10 & 6 \\
\hline P02 & PDS+ & male & 23.42 & 2 & -100.00 & 10 & 6 \\
\hline P03 & PDS+ & male & 29.08 & 3 & 81.81 & 12 & 2 \\
\hline P04 & PDS+ & male & 27.83 & 3 & 100.00 & 12 & 4 \\
\hline P05 & PDS+ & male & 24.08 & 2 & 100.00 & 12 & 5 \\
\hline P06 & PDS+ & male & 30.00 & 2 & 80.00 & 11 & 9 \\
\hline P07 & PDS+ & male & 15.92 & 2 & -100.00 & 12 & 4 \\
\hline P08 & PDS+ & male & 18.50 & 3 & 100.00 & 12 & 5 \\
\hline P09 & PDS+ & male & 20.75 & 4 & 66.66 & 12 & 3 \\
\hline P10 & PDS+ & male & 27.25 & 5 & 100.00 & 11 & 3 \\
\hline P11 & PDS+ & female & 17.92 & 3 & 100.00 & 12 & 2 \\
\hline P12 & PDS+ & male & 15.42 & 1 & 81.81 & 12 & 3 \\
\hline P13 & PDS+ & male & 27.92 & 2 & 100.00 & 12 & 2 \\
\hline P14 & PDS+ & female & 15.75 & 1 & 100.00 & 11 & 13 \\
\hline P15 & PDS+ & male & 18.58 & 3 & 81.81 & 11 & 4 \\
\hline P16 & PDS+ & male & 14.42 & 1 & 100.00 & 12 & 2 \\
\hline P17 & PDS+ & male & 15.92 & 1 & 81.81 & 10 & 4 \\
\hline P18 & PDS+ & male & 53.67 & 6 & 100.00 & 13 & 12 \\
\hline P19 & PDS+ & male & 21.00 & 2 & 100.00 & 11 & 4 \\
\hline P20 & PDS+ & male & 31.58 & 6 & 81.81 & 12 & 2 \\
\hline P21 & PDS+ & male & 57.00 & 2 & 100.00 & 14 & 6 \\
\hline P22 & PDS+ & male & 16.67 & 2 & 66.60 & 10 & 4 \\
\hline P23 & PDS- & male & 37.50 & 2 & 100.00 & 11 & 2.5 \\
\hline P24 & PDS- & male & 28.33 & 6 & 100.00 & 10 & 2 \\
\hline P25 & PDS- & male & 28.17 & 6 & 100.00 & 12 & 6 \\
\hline P26 & PDS- & male & 25.58 & 3 & 100.00 & 10 & 2 \\
\hline P27 & PDS- & male & 34.17 & 6 & 63.63 & 11 & 2 \\
\hline P28 & PDS- & male & 27.92 & 3 & 53.84 & 10 & 6 \\
\hline P29 & PDS- & female & 27.50 & 6 & 66.60 & 15 & 3 \\
\hline P30 & PDS- & male & 35.58 & 6 & 81.81 & 15 & 3 \\
\hline P31 & PDS- & male & 46.75 & 6 & 81.82 & 12 & 2 \\
\hline P32 & PDS- & male & 43.33 & 6 & 100.00 & 11 & 3 \\
\hline P33 & PDS- & male & 34.25 & 3 & 81.81 & 12 & 2 \\
\hline P34 & PDS- & male & 30.42 & 6 & 100.00 & 11 & 10 \\
\hline P35 & PDS- & male & 46.33 & 7 & 100.00 & 12 & 14 \\
\hline
\end{tabular}




\section{Appendices}

Appendix C: Fluent speech: neural basis of sensorimotor plasticity in developmental stuttering

\begin{tabular}{|c|c|c|c|c|c|c|c|}
\hline P36 & PDS- & male & 43.50 & 7 & 100.00 & 11 & 2 \\
\hline P37 & PDS- & male & 34.58 & 2 & -20.00 & 12 & 5 \\
\hline P38 & PDS- & male & 33.33 & 6 & 66.60 & 11 & 7 \\
\hline P39 & PDS- & female & 27.17 & 3 & 100.00 & 11 & 10 \\
\hline P40 & PDS- & male & 42.50 & 5 & 66.60 & 11 & 8 \\
\hline P41 & $\mathrm{HC}$ & male & 31.67 & 6 & 81.81 & 11 & \\
\hline P42 & $\mathrm{HC}$ & male & 34.75 & 2 & 81.81 & 12 & \\
\hline P43 & $\mathrm{HC}$ & male & 23.50 & 5 & -66.60 & 11 & \\
\hline P44 & $\mathrm{HC}$ & female & 19.92 & 3 & 100.00 & 11 & \\
\hline P45 & $\mathrm{HC}$ & male & 27.75 & 3 & 100.00 & 14 & \\
\hline P46 & $\mathrm{HC}$ & male & 20.25 & 3 & 100.00 & 13 & \\
\hline P47 & $\mathrm{HC}$ & male & 28.33 & 6 & 100.00 & 12 & \\
\hline P48 & $\mathrm{HC}$ & male & 27.08 & 3 & 60.00 & 12 & \\
\hline P49 & $\mathrm{HC}$ & male & 24.08 & 5 & 66.60 & 12 & \\
\hline P50 & $\mathrm{HC}$ & male & 30.08 & 6 & 66.60 & 12 & \\
\hline P51 & $\mathrm{HC}$ & male & 20.67 & 3 & 81.81 & 11 & \\
\hline P52 & $\mathrm{HC}$ & male & 22.42 & 5 & 66.66 & 11 & \\
\hline P53 & $\mathrm{HC}$ & female & 17.00 & 2 & 100.00 & 12 & \\
\hline P54 & $\mathrm{HC}$ & male & 30.58 & 6 & 100.00 & 11 & \\
\hline P55 & $\mathrm{HC}$ & female & 16.83 & 1 & -66.60 & 11 & \\
\hline P56 & $\mathrm{HC}$ & female & 23.42 & 5 & -54.00 & 11 & \\
\hline P57 & $\mathrm{HC}$ & male & 27.00 & 4 & 42.80 & 11 & \\
\hline P58 & $\mathrm{HC}$ & male & 52.42 & 3 & 100.00 & 10 & \\
\hline P59 & $\mathrm{HC}$ & male & 27.00 & 5 & 100.00 & 11 & \\
\hline P60 & $\mathrm{HC}$ & male & 14.17 & 1 & 100.00 & 11 & \\
\hline P61 & $\mathrm{HC}$ & male & 18.67 & 2 & 100.00 & 12 & \\
\hline P62 & $\mathrm{HC}$ & male & 17.42 & 2 & 81.81 & 11 & \\
\hline P63 & $\mathrm{HC}$ & male & 17.67 & 2 & 100.00 & 11 & \\
\hline P64 & $\mathrm{HC}$ & male & 27.92 & 3 & 100.00 & 11 & \\
\hline P65 & $\mathrm{HC}$ & male & 26.50 & 3 & 81.81 & 11 & \\
\hline P66 & $\mathrm{HC}$ & male & 25.25 & 5 & 100.00 & 11 & \\
\hline P67 & $\mathrm{HC}$ & male & 26.75 & 4 & 100.00 & 11 & \\
\hline P68 & $\mathrm{HC}$ & male & 23.08 & 3 & 100.00 & 11 & \\
\hline
\end{tabular}


7. Appendices

Appendix C: Fluent speech: neural basis of sensorimotor plasticity in developmental stuttering

Table 2: Total scores of SSI and OASES at T1 and T2 per participant.

\begin{tabular}{|c|c|c|c|c|c|}
\hline ID & Group & $\begin{array}{c}\text { T1 SSI-4 } \\
\text { (totals score) }\end{array}$ & $\begin{array}{c}\text { T2 SSI-4 } \\
\text { (total score) }\end{array}$ & $\begin{array}{c}\text { T1 OASES } \\
\text { (total score) }\end{array}$ & $\begin{array}{c}\text { T2 OASES } \\
\text { (total score) }\end{array}$ \\
\hline P01 & PDS+ & 28 & 17 & 3.17 & 1.79 \\
\hline P02 & PDS+ & 16 & 4 & 3.18 & 1.53 \\
\hline P03 & PDS+ & 23 & 15 & 2.07 & 1.91 \\
\hline P04 & PDS+ & 27 & 5 & 3.11 & 1.66 \\
\hline P05 & PDS+ & 38 & 30 & 3.23 & 2.13 \\
\hline P06 & PDS+ & 13 & 6 & 2.52 & 2.05 \\
\hline P07 & PDS+ & 22 & 9 & *2.65 & ${ }^{*} 1.51$ \\
\hline P08 & PDS+ & 13 & 8 & 2.96 & 2.13 \\
\hline P09 & PDS+ & 19 & 7 & 3.00 & 1.83 \\
\hline P10 & PDS+ & 10 & 5 & 2.18 & 1.63 \\
\hline P11 & PDS+ & 18 & 5 & 2.33 & 2.06 \\
\hline P12 & PDS+ & 39 & 37 & *2.69 & ${ }^{*} 2.33$ \\
\hline P13 & PDS+ & 30 & 17 & 2.74 & 2.48 \\
\hline P14 & PDS+ & 7 & 5 & *2.51 & *1.41 \\
\hline P15 & PDS+ & 24 & 2 & 3.18 & 2.08 \\
\hline P16 & PDS+ & 31 & 11 & ${ }^{*} 2.87$ & ${ }^{*} 2.24$ \\
\hline P17 & PDS+ & 26 & 15 & *3.21 & *1.65 \\
\hline P18 & PDS+ & 32 & 12 & 2.99 & 1.81 \\
\hline P19 & PDS+ & 28 & 1 & 3.10 & 1.22 \\
\hline P20 & PDS+ & 32 & 9 & 3.19 & 3.02 \\
\hline P21 & PDS+ & 33 & 22 & 3.64 & 2.15 \\
\hline P22 & PDS+ & 11 & 9 & *3.59 & *1.89 \\
\hline P23 & PDS- & 28 & 26 & 2.99 & 2.72 \\
\hline P24 & PDS- & 7 & 8 & 1.38 & 1.27 \\
\hline P25 & PDS- & 5 & 8 & 1.58 & 1.67 \\
\hline P26 & PDS- & 15 & 13 & 1.30 & 1.27 \\
\hline P27 & PDS- & 4 & 4 & 1.89 & 1.87 \\
\hline P28 & PDS- & 22 & 15 & 2.04 & 1.80 \\
\hline P29 & PDS- & 14 & 12 & 2.21 & 2.25 \\
\hline P30 & PDS- & 16 & 18 & 2.13 & 2.45 \\
\hline P31 & PDS- & 42 & 44 & 2.25 & 2.17 \\
\hline P32 & PDS- & 7 & 10 & 2.08 & 1.91 \\
\hline P33 & PDS- & 20 & 22 & 1.73 & 1.71 \\
\hline P34 & PDS- & 10 & 4 & 2.16 & 2.20 \\
\hline P35 & PDS- & 11 & 11 & 1.86 & 1.92 \\
\hline P36 & PDS- & 32 & 35 & 2.46 & 2.28 \\
\hline
\end{tabular}




\section{Appendices}

Appendix C: Fluent speech: neural basis of sensorimotor plasticity in developmental stuttering

\begin{tabular}{llllll} 
P37 & PDS- & 5 & 3 & 1.83 & 1.69 \\
P38 & PDS- & 14 & 16 & 2.44 & 2.13 \\
P39 & PDS- & 15 & 16 & 1.10 \\
P40 & PDS- & 11 & 10 & 1.80 \\
P41 & HC & 0 & 5 & \\
P42 & HC & 2 & 0 & \\
P43 & HC & 0 & 0 & \\
P44 & HC & 0 & 0 & \\
P45 & HC & 0 & 0 & \\
P46 & HC & 5 & 0 & \\
P47 & HC & 1 & 0 & \\
P48 & HC & 0 & 0 & \\
P49 & HC & 0 & 0 & \\
P50 & HC & 5 & 0 & \\
P51 & HC & 4 & 2 & \\
P52 & HC & 0 & 0 & \\
P53 & HC & 4 & 2 & \\
P54 & HC & 4 & 0 & \\
P55 & HC & 0 & 0 & \\
P56 & HC & 0 & 0 & \\
P57 & HC & 5 & 0 & \\
P58 & HC & 0 & 0 & \\
P59 & HC & 0 & 0 & \\
P60 & HC & 0 & 0 & \\
P61 & HC & 4 & 0 & \\
P62 & HC & 7 & 0 & \\
P63 & HC & 8 & 0 & \\
P64 & HC & 0 & 0 & \\
P65 & HC & 0 & 0 & \\
P66 & HC & 0 & 0 & \\
P67 & HC & 0 & 0 & \\
P68 & HC & 2 & 0 & \\
\hline & He. & 0 & \\
\end{tabular}

Note. PDS+ = persons with developmental stuttering who participated in the intensive intervention. PDS- $=$ persons with developmental stuttering not taking part in the intensive intervention. $\mathrm{HC}=$ healthy controls. SSI = stuttering severity index. OASES = Assessment of the Speaker's Experience of stuttering. $\mathrm{T} 1=$ first measurement prior to intervention; $\mathrm{T} 2=$ second measurement after intervention; * OASES-T = Assessment of the Speaker's Experience of stuttering for teenagers (Yaruss \& Quesal, 2006). 


\section{Appendices}

Appendix C: Fluent speech: neural basis of sensorimotor plasticity in developmental stuttering

Table 3. Brain hubs of speech (motor) planning.

\begin{tabular}{lllll}
\hline Brain hub - anatomical label & ROI Label & X & Y & Z \\
\hline Inferior frontal gyrus, area 44 & L IFG & -56 & 8 & 21 \\
Parietal operculum & L OP & -54 & -22 & 22 \\
Inferior parietal lobe & L IPL & -54 & -36 & 22 \\
Inferior parietal lobe & R IPL & 48 & -56 & 32 \\
Middle temporal gyrus & L MTG & -58 & 0 & -30 \\
\hline
\end{tabular}

Note. All coordinates refer to $\mathrm{MNI}$-space. $\mathrm{L}=$ left, $\mathrm{R}=$ right.

Coordinates were derived from (Neef et al., 2016) and (Clos et al., 2013).

Table 4. Brain hubs of inhibition.

\begin{tabular}{lllll}
\hline Brain hub - anatomical label & ROI Label & X & Y & Z \\
\hline Inferior frontal gyrus, area 44 & R IFG & 50 & 18 & 6 \\
Supplementary motor area $^{1}$ & R SMA & 6 & 18 & 48 \\
Insula $^{2}$ & R In & 36 & 18 & 0 \\
rostral cingulate zone $^{3}$ (median cingulate paracingulate) & R rCC & 4 & 26 & 38 \\
Subthalamic nucleus & R STN & 8 & -13 & 7
\end{tabular}

Note. All coordinates refer to $\mathrm{MNI}$-space. $\mathrm{L}=$ left, $\mathrm{R}=$ right. ${ }^{4}$ Coordinates were derived from (Zhang et al., 2017); Coordinates were derived from STN-Atlas Forstmann (Keuken \& Forstmann, 2015). 


\section{Appendices}

Appendix C: Fluent speech: neural basis of sensorimotor plasticity in developmental stuttering

Table 5. Brain hubs of articulatory convergence.

\begin{tabular}{lllll}
\hline Brain hub - anatomical label & ROI Label & X & Y & Z \\
\hline Supplementary motor area & L SMA & -3 & -1 & 57 \\
Supplementary motor area & R SMA & 4 & -6 & 64 \\
Precentral gyrus, & L PrCG & -50 & -4 & 35 \\
Ventral precentral gyrus & R vPrCG & 53 & -7 & 36 \\
Precentral gyrus & R PrCG & 59 & 3 & 19 \\
Postcentral gyrus & L PoCG & -46 & -15 & 15 \\
Putamen & L Put & -25 & -4 & -3 \\
Putamen & R Put & 24 & -2 & 0 \\
Thalamus & L Th & -12 & -16 & 1 \\
Thalamus & R Th & 15 & -15 & 1 \\
\hline
\end{tabular}

Note. All coordinates refer to MNI-space. $\mathrm{L}=$ left, $\mathrm{R}=$ right.

Coordinates were derived from (Guenther, 2016).

Table 6. Summary of changes in behavioral outcome measures.

\begin{tabular}{lcccccc}
\hline & $\begin{array}{c}\text { Intervention group } \\
\end{array}$ & \multicolumn{2}{c}{$\begin{array}{c}\text { Stuttering controls } \\
\text { T1 }\end{array}$} & T2 & T1 & Thent controls \\
& T1 & & T2 & T1 & T2 \\
\hline SSI-4 total & $25(14.3)$ & $9(10.0)$ & $14(11.3)$ & $13(9.0)$ & $0(4.0)$ & $0(2.3)$ \\
OASES total & $3(0.5)$ & $2(0.5)$ & $2(0.4)$ & $2(0.5)$ & - & - \\
\hline Note. Ordinal-scaled variables are presented as median (interquartile range).
\end{tabular}




\section{Appendices}

Appendix C: Fluent speech: neural basis of sensorimotor plasticity in developmental stuttering

Table 7. Posthoc comparisons on results of global ANCOVA differentiating the age effect between groups.

\begin{tabular}{lllllc}
\hline Seed - Target regions & Group comparison & Beta & Df & T value & $p$ \\
\hline left IFG - left LMC & PDS+ vs. HC & -0.005 & 46 & -1.28 & .207 \\
& PDS+ vs. PDS- & 0.004 & 36 & 0.75 & .462 \\
& PDS- vs. HC & -0.002 & 42 & -0.27 & .788 \\
left IFG - right pSTG & PDS+ vs. HC & -0.001 & 46 & -0.19 & .854 \\
& PDS+ vs. PDS- & -0.003 & 36 & -0.57 & .569 \\
& PDS- vs. HC & -0.004 & 42 & -0.56 & .578 \\
left IFG - left SMG & PDS+ vs. HC & 0.001 & 46 & 0.21 & .837 \\
& PDS+ vs. PDS- & 0.013 & 36 & 2.16 & .038 \\
& PDS- vs. HC & 0.014 & 42 & 2.07 & .045
\end{tabular}

Note. IFG $=$ inferior frontal gyrus. $\mathrm{LMC}=$ laryngeal motor cortex. $\mathrm{pSTG}=$ posterior superior temporal gyrus. $\mathrm{SMG}=$ supramarginal gyrus. PDS $+=$ persons with developmental stuttering who participated in the intensive intervention. PDS- = persons with developmental stuttering not taking part in the intensive intervention. $\mathrm{HC}=$ healthy controls. 


\section{Appendices}

Appendix C: Fluent speech: neural basis of sensorimotor plasticity in developmental stuttering

Table 8. Group averages and test statistics of one-sample paired t-tests for the change of diffusion parameters in PDS+.

\begin{tabular}{|c|c|c|c|c|c|c|}
\hline PDS+ & & Mean & $S D$ & $t$ & $p$ & $n$ \\
\hline & & - & & & & \\
\hline \multirow[t]{5}{*}{ LMC_L } & $\mathrm{FA}$ & 0.006111918 & 0.0378503 & -0.757 & .457 & 22 \\
\hline & MD & $-2.42 \mathrm{E}-06$ & $5.48 \mathrm{E}-05$ & -0.207 & .838 & 22 \\
\hline & $\mathrm{RD}$ & 2.36E-06 & $6.08 \mathrm{E}-05$ & 0.182 & .857 & 22 \\
\hline & $A D$ & $-1.20 \mathrm{E}-05$ & 4.96E-05 & -1.135 & .269 & 22 \\
\hline & & - & & & & \\
\hline \multirow[t]{4}{*}{ LMC_R } & $\mathrm{FA}$ & 0.002392849 & 0.02024007 & -0.542 & .594 & 21 \\
\hline & MD & 8.49E-07 & $1.24 \mathrm{E}-01$ & 0.124 & .903 & 21 \\
\hline & $\mathrm{RD}$ & 2.16E-06 & $3.26 \mathrm{E}-05$ & 0.305 & .764 & 21 \\
\hline & $A D$ & $-1.78 \mathrm{E}-06$ & $3.23 \mathrm{E}-05$ & -0.253 & .803 & 21 \\
\hline \multirow[t]{4}{*}{ SLF_L } & $\mathrm{FA}$ & 0.001307349 & 0.01688303 & -0.346 & .733 & 20 \\
\hline & MD & $-5.83 E-06$ & 2.96E-05 & -0.879 & .390 & 20 \\
\hline & $\mathrm{RD}$ & $-3.79 E-06$ & $2.88 \mathrm{E}-05$ & -0.588 & .564 & 20 \\
\hline & $A D$ & $-9.91 E-06$ & $3.55 E-05$ & -1.249 & .227 & 20 \\
\hline \multirow[t]{4}{*}{ SLF_R } & $\mathrm{FA}$ & 0.003997726 & 0.02036064 & -0.921 & .368 & 22 \\
\hline & MD & $-2.30 \mathrm{E}-06$ & 3.57E-05 & -0.302 & .766 & 22 \\
\hline & $\mathrm{RD}$ & $-1.62 E-06$ & 3.36E-05 & -0.226 & .824 & 22 \\
\hline & $A D$ & $-3.66 \mathrm{E}-06$ & 4.36E-05 & -0.393 & .698 & 22 \\
\hline & & - & & & & \\
\hline \multirow[t]{4}{*}{ FAT_L } & $\mathrm{FA}$ & 0.002283435 & 0.01474724 & -0.710 & .486 & 21 \\
\hline & MD & $-2.25 E-06$ & 2.38E-05 & -0.432 & .670 & 21 \\
\hline & $\mathrm{RD}$ & $-1.01 E-06$ & 2.25E-05 & -0.205 & .839 & 21 \\
\hline & $A D$ & $-4.72 E-06$ & 2.89E-05 & -0.749 & .463 & 21 \\
\hline \multirow[t]{4}{*}{ FAT_R } & $\mathrm{FA}$ & 0.001579629 & 0.01562759 & 0.474 & .640 & 22 \\
\hline & MD & $-8.39 E-06$ & 2.24E-05 & -1.759 & .093 & 22 \\
\hline & $\mathrm{RD}$ & $-6.76 \mathrm{E}-06$ & $2.28 \mathrm{E}-05$ & -1.393 & .178 & 22 \\
\hline & $A D$ & $-1.17 \mathrm{E}-05$ & $2.50 \mathrm{E}-05$ & -2.189 & .040 & 22 \\
\hline
\end{tabular}

Note. $\mathrm{LMC}=$ tract connecting the laryngeal motor cortex with the inferior frontal gyrus. SLF $=$ superior longitudinal fasciculus. $\mathrm{FAT}=$ frontal aslant tract. $\mathrm{FA}=$ fractional anisotropy. $\mathrm{MD}=$ mean diffusivity. $R D=$ radial diffusivity. $A D=$ axial diffusivity. $L=$ left. $R=$ right. 


\section{Appendices}

Appendix C: Fluent speech: neural basis of sensorimotor plasticity in developmental stuttering

Table 9. Group averages and test statistics of one-sample paired t-tests for the change of diffusion parameters in PDS-.

\begin{tabular}{|c|c|c|c|c|c|c|}
\hline PDS- & & Mean & $S D$ & $t$ & $p$ & $n$ \\
\hline \multirow[t]{4}{*}{ LMC_L } & FA & 0.00101291 & 0.0102805 & 0.418 & .681 & 18 \\
\hline & MD & $-1.00 \mathrm{E}-06$ & 2.56E-05 & -0.166 & .870 & 18 \\
\hline & $\mathrm{RD}$ & -1.57E-06 & 2.35E-05 & -0.283 & .781 & 18 \\
\hline & $A D$ & 1.32E-07 & 3.23E-05 & 0.017 & .986 & 18 \\
\hline \multirow[t]{4}{*}{ LMC_R } & $\mathrm{FA}$ & 0.01003447 & 0.01405517 & -2.856 & .012 & 16 \\
\hline & MD & $-3.70 \mathrm{E}-07$ & 2.24E-05 & -0.066 & .948 & 16 \\
\hline & $\mathrm{RD}$ & 4.80E-06 & 2.24E-05 & 0.857 & .405 & 16 \\
\hline & $A D$ & -1.07E-05 & $2.74 \mathrm{E}-05$ & -1.563 & .139 & 16 \\
\hline \multirow[t]{5}{*}{ SLF_L } & $\mathrm{FA}$ & 0.00594962 & 0.01273219 & -1.983 & .064 & 18 \\
\hline & MD & $1.01 \mathrm{E}-05$ & 2.13E-05 & 2.015 & .060 & 18 \\
\hline & $\mathrm{RD}$ & $1.00 \mathrm{E}-05$ & 2.09E-05 & 2.036 & .058 & 18 \\
\hline & $A D$ & 1.03E-05 & 2.54E-05 & 1.716 & .104 & 18 \\
\hline & & - & & & & \\
\hline \multirow[t]{5}{*}{ SLF_R } & FA & 0.00036577 & 0.01170426 & -0.129 & .899 & 17 \\
\hline & MD & $-4.82 \mathrm{E}-06$ & 1.52E-05 & -1.306 & .210 & 17 \\
\hline & $\mathrm{RD}$ & $-3.36 E-06$ & 1.62E-05 & -0.853 & .406 & 17 \\
\hline & $A D$ & -7.74E-06 & 1.71E-05 & -1.864 & .081 & 17 \\
\hline & & - & & & & \\
\hline \multirow[t]{5}{*}{ FAT_L } & FA & 0.00200257 & 0.01143862 & -0.722 & .481 & 17 \\
\hline & MD & 3.06E-06 & 1.53E-05 & 0.823 & .423 & 17 \\
\hline & $\mathrm{RD}$ & 3.63E-06 & $1.59 \mathrm{E}-05$ & 0.944 & .359 & 17 \\
\hline & $A D$ & 1.92E-06 & 1.56E-05 & 0.505 & .620 & 17 \\
\hline & & - & & & & \\
\hline \multirow[t]{4}{*}{ FAT_R } & $\mathrm{FA}$ & 0.00517892 & 0.00883776 & -2.344 & .033 & 16 \\
\hline & MD & 1.16E-06 & 1.35E-05 & 0.342 & .737 & 16 \\
\hline & $\mathrm{RD}$ & $3.46 \mathrm{E}-06$ & 1.34E-05 & 1.034 & .318 & 16 \\
\hline & $A D$ & $-3.44 \mathrm{E}-06$ & 1.70E-05 & -0.811 & .430 & 16 \\
\hline
\end{tabular}

Note. $\mathrm{LMC}=$ tract connecting the laryngeal motor cortex with the inferior frontal gyrus. SLF $=$ superior longitudinal fasciculus. $\mathrm{FAT}=$ frontal aslant tract. $\mathrm{FA}=$ fractional anisotropy. $\mathrm{MD}=$ mean diffusivity . $R D=$ radial diffusivity. $A D=$ axial diffusivity $. L=$ left. $R=$ right . 
7. Appendices

Appendix C: Fluent speech: neural basis of sensorimotor plasticity in developmental stuttering

Table 10. Group averages and test statistics of one-sample paired t-tests for the change of diffusion parameters in $\mathrm{HC}$.

\begin{tabular}{|c|c|c|c|c|c|c|}
\hline HC & & Mean & $S D$ & $t$ & $p$ & $n$ \\
\hline & & - & & & & \\
\hline \multirow[t]{5}{*}{ LMC_L } & FA & 0.00736401 & 0.0157854 & -2.379 & .025 & 26 \\
\hline & MD & $2.15 \mathrm{E}-06$ & 2.70E-05 & 0.406 & .688 & 26 \\
\hline & $\mathrm{RD}$ & 4.45E-06 & 2.69E-05 & 0.842 & .408 & 26 \\
\hline & $A D$ & $-2.45 E-06$ & $3.10 \mathrm{E}-05$ & -0.402 & .691 & 26 \\
\hline & & - & & & & \\
\hline \multirow[t]{4}{*}{ LMC_R } & FA & 0.00208867 & 0.01715778 & -0.596 & .557 & 24 \\
\hline & MD & -7.57E-06 & $1.83 \mathrm{E}-05$ & -2.028 & .054 & 24 \\
\hline & $\mathrm{RD}$ & $-4.41 \mathrm{E}-06$ & $1.98 \mathrm{E}-05$ & -1.092 & .286 & 24 \\
\hline & $A D$ & $-1.39 \mathrm{E}-05$ & 2.04E-05 & -3.333 & .003 & 24 \\
\hline \multirow[t]{4}{*}{ SLF_L } & FA & 0.00898542 & 0.01057398 & -4.333 & .000 & 26 \\
\hline & MD & 7.57E-06 & 2.00E-05 & 1.925 & .066 & 26 \\
\hline & $\mathrm{RD}$ & $9.75 \mathrm{E}-06$ & 1.84E-05 & 2.706 & .012 & 26 \\
\hline & $A D$ & $3.21 \mathrm{E}-06$ & $2.59 \mathrm{E}-05$ & 0.632 & .533 & 26 \\
\hline \multirow[t]{4}{*}{ SLF_R } & FA & 0.00800339 & 0.01273894 & -3.078 & .005 & 24 \\
\hline & MD & $2.99 \mathrm{E}-06$ & $1.62 \mathrm{E}-05$ & 0.903 & .376 & 24 \\
\hline & $\mathrm{RD}$ & $6.25 \mathrm{E}-06$ & $1.74 \mathrm{E}-05$ & 1.757 & .092 & 24 \\
\hline & $A D$ & $-3.55 E-06$ & 1.74E-05 & -0.999 & .328 & 24 \\
\hline \multirow[t]{4}{*}{ FAT_L } & FA & 0.00513031 & 0.01772636 & -1.476 & .152 & 26 \\
\hline & MD & $1.64 \mathrm{E}-06$ & $1.77 \mathrm{E}-05$ & 0.474 & .639 & 26 \\
\hline & $\mathrm{RD}$ & 4.30E-06 & 2.06E-05 & 1.063 & .298 & 26 \\
\hline & $A D$ & -3.67E-06 & 2.05E-05 & -0.914 & .369 & 26 \\
\hline \multirow[t]{4}{*}{ FAT_R } & $\mathrm{FA}$ & -0.0038835 & 0.01133259 & -1.747 & .093 & 26 \\
\hline & MD & 2.29E-06 & $2.20 \mathrm{E}-05$ & 0.531 & .600 & 26 \\
\hline & $\mathrm{RD}$ & $3.44 \mathrm{E}-06$ & 1.79E-05 & 0.979 & .337 & 26 \\
\hline & $A D$ & -8.97E-09 & 3.32E-05 & -0.001 & .999 & 26 \\
\hline
\end{tabular}

Note. $\mathrm{LMC}=$ tract connecting the laryngeal motor cortex with the inferior frontal gyrus. SLF $=$ superior longitudinal fasciculus. $\mathrm{FAT}=$ frontal aslant tract. $\mathrm{FA}=$ fractional anisotropy. $\mathrm{MD}=$ mean diffusivity . $R D=$ radial diffusivity. $A D=$ axial diffusivity $. L=$ left. $R=$ right . 


\section{Appendices}

Appendix C: Fluent speech: neural basis of sensorimotor plasticity in developmental stuttering

Figure 1. Probability maps, waypoint and exclusion masks for the segmentation of the tract connecting LMC and IFG.

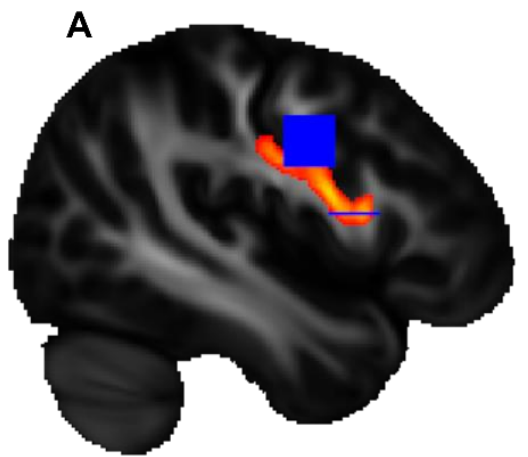

$x=-45$

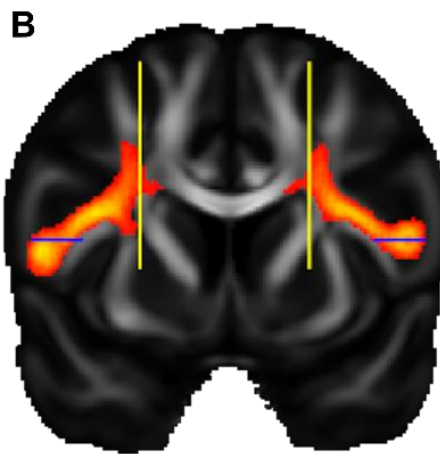

$y=8$

Waypoint mask $\quad$ Exclusion mask

Tract probability $0.25-1$

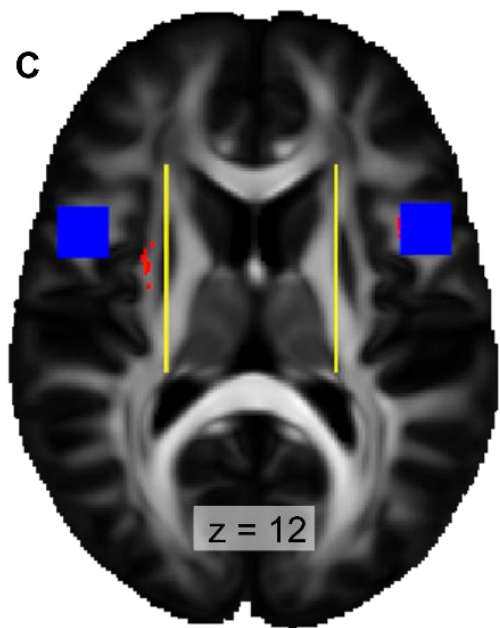

Note. Waypoint masks were manually drawn on the FSL_HCP1065_FA_1mm standard template overlaid with custom tract-specific probability maps. Masks are shown in the sagittal (A); coronal (B) and axial plane $(\mathrm{C})$.

Figure 2. Results of the global ROI-to-ROI resting-state connectivity analysis.
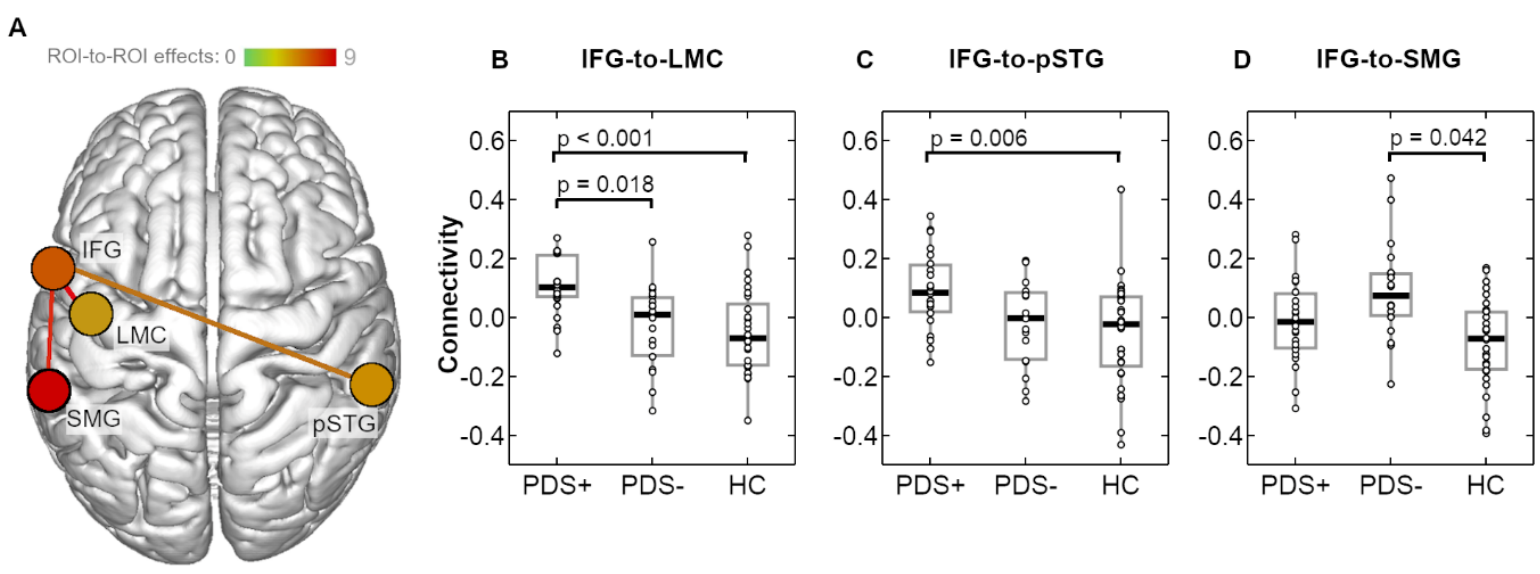

Note. (A) The global ANCOVA revealed significant Group $\times$ Time interactions for left IFG to left LMC, left IFG to left SMG, and left IFG to right PSTG (color coded F-statistics). (B) Boxplots display the change of connection strength as given by the difference of functional connectivity (T2-T1) for the three study groups for left IFG to left LMC, (C) left IFG to right PSTG, and (D) left IFG to left SMG. $I F G=$ inferior frontal gyrus. $L M C=$ laryngeal motor cortex. $\mathrm{pSTG}=$ posterior superior temporal gyrus. $S M G=$ supramarginal gyrus . 


\section{Appendices}

Appendix C: Fluent speech: neural basis of sensorimotor plasticity in developmental stuttering

Figure 3. Association between left IFG to left SMG connectivity change from T1 to T2 and age differentiated by group.
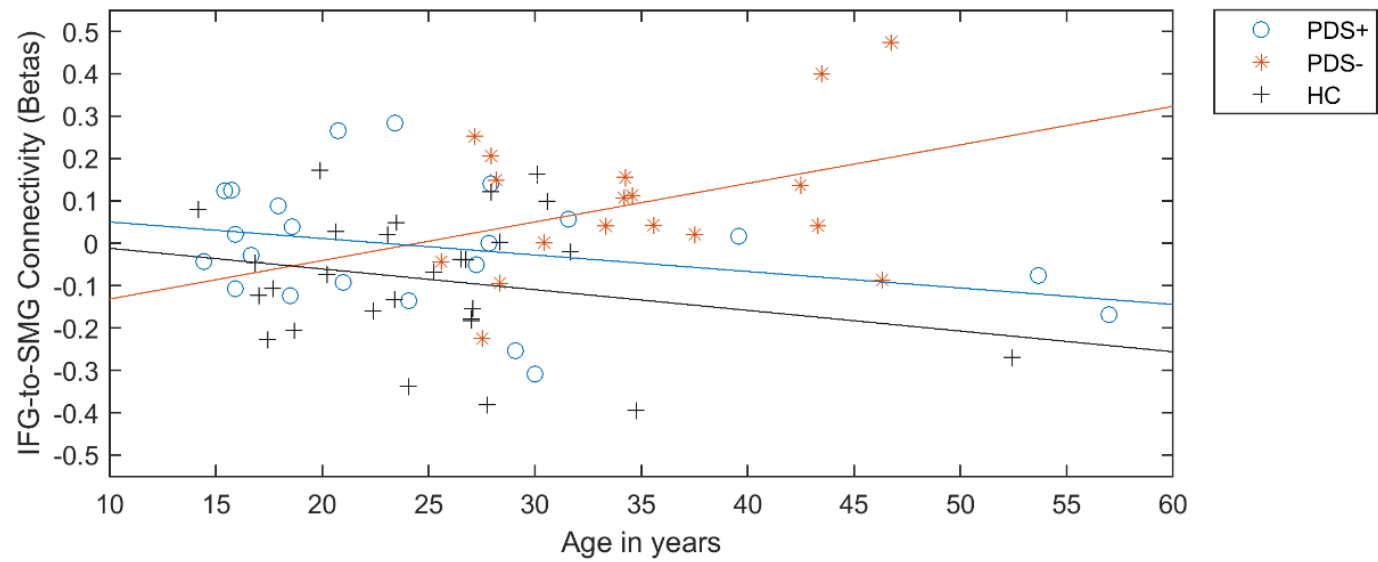

Note. Lines represent least-square regression lines on each scatter plot. Pearson correlations between age and connectivity change did not reach significance: $\mathrm{rPDS}_{+}=-0.305, \mathrm{rPDS}_{\mathrm{P}}=0.375$, $\mathrm{r}_{\mathrm{HC}}=-0.234$, all $\mathrm{p}>.05$. Connectivity values were extracted from the global ANCOVA with correction for multiple comparisons of seeds. 
8. Declaration of Contribution

\section{Declaration of Contribution}

Hereby, I declare that I wrote this thesis independently and with no other sources and aids than quoted. Assistance of third parties was only accepted if scientifically justifiable and acceptable in regards to the examination regulations. All sources have been quoted.

Parts of this thesis and some figures have been used in the following article:

Korzeczek, A., Primassin, A., Wolff von Gudenberg, A., Dechent, P., Paulus, W., Sommer, M., \& Neef, N. E. (2020). Fluent speech: neural basis of sensorimotor plasticity in developmental stuttering. BioRxiv. Advance online publication.

https://doi.org/10.1101/2020.07.27.219360

Alexandra Korzeczek

Göttingen, January 2021 